Volume 111, Number 2 Summer 2011 ISSN 0042-8639

The Volta Review Alexander Graham Bell Association for the Deaf and Hard of Hearing

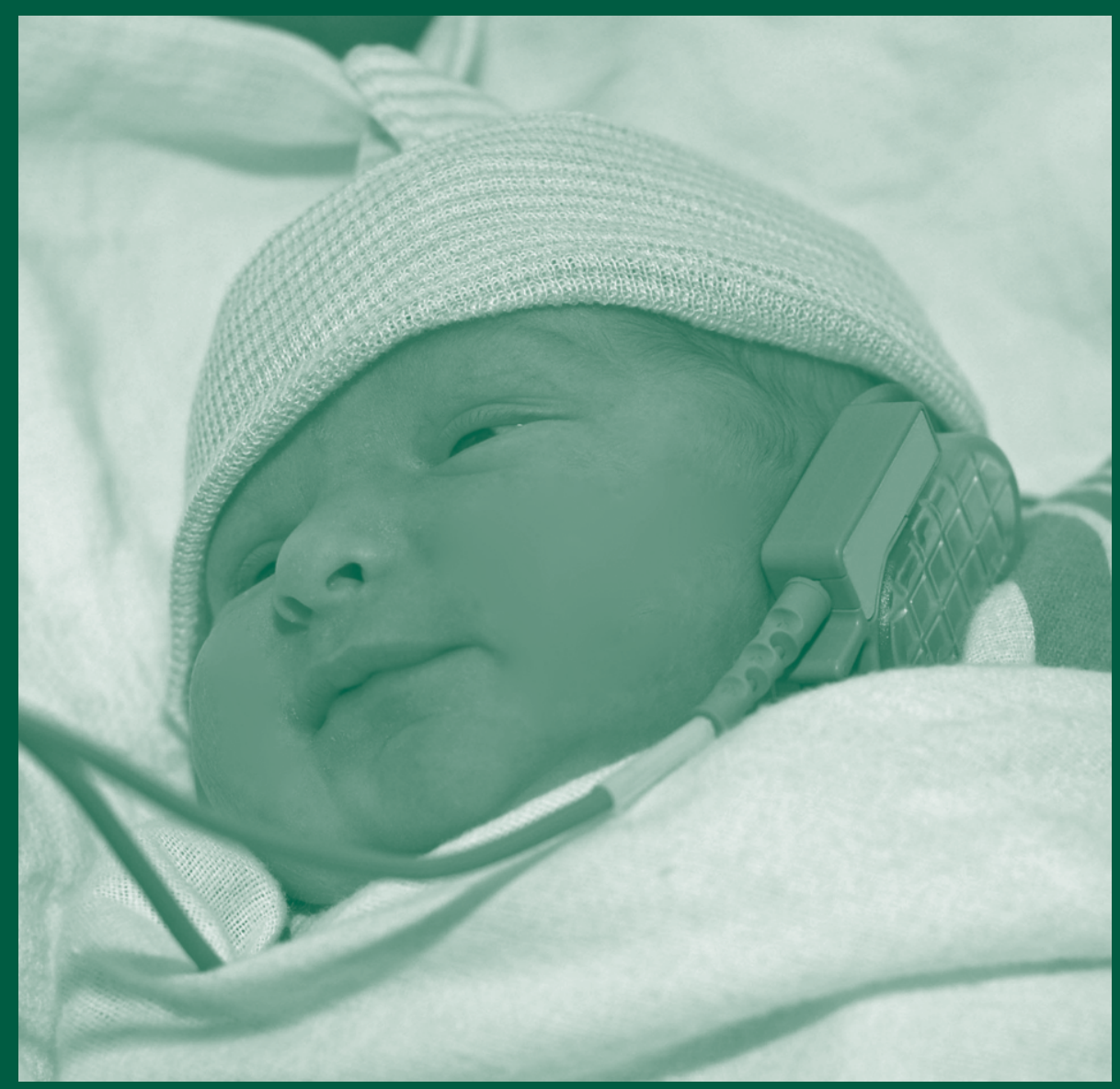

A Strategic Analysis of State Early Hearing Detection and Intervention Programs

Edited by

Jackson Roush, Ph.D. 


\section{The Volta Review}

Volume 111, Number 2

Summer 2011

ISSN 0042-8639

The Alexander Graham Bell Association for the Deaf and Hard of Hearing helps families, health care providers and education professionals understand childhood hearing loss and the importance of early diagnosis and intervention. Through advocacy, education, research and financial aid, AG Bell helps to ensure that every child and adult with hearing loss has the opportunity to listen, talk and thrive in mainstream society. With chapters located in the United States and a network of international affiliates, AG Bell supports its mission: Advocating Independence through Listening and Talking!

\section{A Strategic Analysis of State Early Hearing Detection and Intervention Programs}

8I

\section{Loss to Follow-Up: Issues and Recommendations Jeff Hoffman, M.S., CCC-A; Karen F. Muñoz, Ed.D., CCC-A; Tamala S. Bradham, Ph.D., CCC-A; and Lauri Nelson, Ph.D.}

\section{Preface}

Jackson Roush, Ph.D.

\section{Strategic Planning to Improve EHDI Programs}

Karl R.White, Ph.D.; and Kristina M. Blaiser, Ph.D., CCC-SLP

\section{Newborn Hearing Screening: An Analysis of Current Practices}

K. Todd Houston, Ph.D., CCC-SLP, LSLS Cert. AVT;

Tamala S. Bradham, Ph.D., CCC-A; Karen F. Muñoz, Ed.D., CCC-A; and Gayla Hutsell Guignard, M.A., CCC-A/SLP, LSLS Cert. AVT
A Systematic Analysis of Audiological Services in EHDI
Karen F. Muñoz, Ed.D., CCC-A; Tamala S. Bradham, Ph.D., CCC-A; and Lauri Nelson, Ph.D.

\section{The EHDI and Early Intervention Connection \\ Lauri Nelson, Ph.D.; Tamala S. Bradham, Ph.D., CCC-A; and \\ K. Todd Houston, Ph.D., CCC-SLP, LSLS Cert. AVT}

\section{Integrating the Medical Home into the EHDI Process}

Karen F. Muñoz, Ed.D., CCC-A; Lauri Nelson, Ph.D.;

Tamala S. Bradham, Ph.D., CCC-A; Jeff Hoffman, M.S., CCC-A; and

K. Todd Houston, Ph.D., CCC-SLP, LSLS Cert. AVT 
195 Periodic Early Childhood Hearing Screening:

The EHDI Perspective

Jeff Hoffman, M.S., CCC-A;

K. Todd Houston, Ph.D., CCC-SLP, LSLS Cert. AVT;

Karen F. Muñoz, Ed.D., CCC-A; and Tamala S. Bradham, Ph.D., CCC-A

209 Professional Development: Are We Meeting the Needs of State EHDI Programs?

K.Todd Houston, Ph.D., CCC-SLP, LSLS Cert. AVT;

Karen F. Muñoz, Ed.D., CCC-A; and Tamala S. Bradham, Ph.D., CCC-A

225 Is the Infrastructure of EHDI Programs Working?

K. Todd Houston, Ph.D., CCC-SLP, LSLS Cert. AVT;

Jeff Hoffman, M.S., CCC-A; Karen F. Muñoz, Ed.D., CCC-A; and

Tamala S. Bradham, Ph.D., CCC-A

243 Data Management in the EHDI System

Tamala S. Bradham, Ph.D., CCC-A; Jeff Hoffman, M.S., CCC-A; and

K. Todd Houston, Ph.D., CCC-SLP, LSLS Cert. AVT

255 Fostering Quality Improvement in EHDI Programs

Tamala S. Bradham, Ph.D., CCC-A; Jeff Hoffman, M.S., CCC-A;

K. Todd Houston, Ph.D., CCC-SLP, LSLS Cert. AVT; and

Gayla Hutsell Guignard, M.A., CCC-A/SLP, LSLS Cert. AVT

267 Interdisciplinary Collaboration in EHDI Programs

Lauri Nelson, Ph.D.; K. Todd Houston, Ph.D., CCC-SLP, LSLS Cert. AVT;

Jeff Hoffman, M.S., CCC-A; and Tamala S. Bradham, Ph.D., CCC-A

28 I Future Directions for EHDI Programs:

Recommendations from a Strategic Analysis

Tamala S. Bradham, Ph.D., CCC-A; and

K. Todd Houston, Ph.D., CCC-SLP, LSLS Cert. AVT

\section{Regular Features}

289 Information for Contributors to The Volta Review

Permission to Copy: The Alexander Graham Bell Association for the Deaf and Hard of Hearing, as copyright owner of this journal, allows single copies of an article to be made for personal use. This consent does not extend to posting on Web sites or other kinds of copying, such as copying for general distribution, for advertising or promotional purposes, for creating new collective works of any type, or for resale without the express written permission of the publisher. For more information, contact AG Bell at 3417 Volta Place, NW, Washington, DC 20007, email editor@agbell.org, or call (202) 337-5220 (voice) or (202) 337-522I (TTY). 



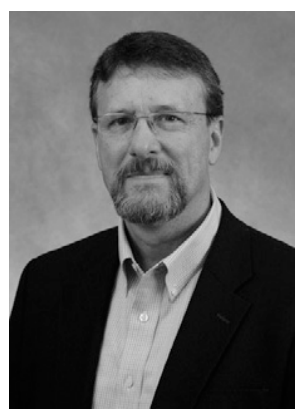

\section{Editor's Preface}

Jackson Roush, Ph.D.,

Guest Editor

Screening of all newborn infants for hearing loss, once viewed as impractical and unrealistic, is now a standard of care throughout the United States. While this is a remarkable achievement in its own right, it is just the first step in a complex, multi-faceted system that includes many organizations and participants. In recent years the term "EHDI" (Early Hearing Detection and Intervention) has been used to describe the full spectrum of organizational resources and clinical services needed to achieve the broader goals of maximizing outcomes for children with hearing loss and their families.

In 2009, the National Center for Hearing Assessment and Management (NCHAM), under the leadership of Dr. Karl White, initiated an ambitious planning process aimed at helping state EHDI program coordinators identify ways to improve their programs. They elected to use a well-established strategic planning tool, "SWOT," to identify strengths, weaknesses, opportunities, and threats. Themes generated from the SWOT analysis were reviewed by a panel of experts and categorized into common themes within several strategic planning areas and used to generate specific recommendations. Drawing on the individual and collective experience of nearly every state EHDI coordinator in the United States, this analysis, the first of its kind, produced an important body of evidence that will be useful to EHDI stakeholders throughout the nation.

It has been a privilege for me to serve as guest editor of this monograph. Thanks and appreciation are due to NCHAM for taking on this challenge, to the 49 state EHDI coordinators whose thoughtful input provided the data for this project, to the 6 authors who analyzed, distilled, and systematically reported the findings and recommendations, and to the numerous reviewers whose expertise contributed substantially to the final product.

Special thanks are due to Drs. Tamala Bradham and Todd Houston for their leadership throughout the project, and to Ms. Melody Felzien for her 
outstanding editorial assistance. We hope the monograph is useful to EHDI programs as they examine their individual strengths and needs, and to those who seek additional resources to expand and improve EHDI programs at all levels.

Sincerely,

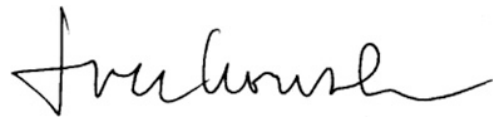

Jackson Roush, Ph.D.

Professor and Director, Division of Speech and Hearing Sciences School of Medicine, University of North Carolina at Chapel Hill 
The Volta Review, Volume 111(2), Summer 2011, 83-108

\title{
Strategic Planning to Improve EHDI Programs
}

\author{
Karl R. White, Ph.D.; and Kristina M. Blaiser, Ph.D., CCC-SLP
}

Because newborn hearing screening has become the standard of care in the United States, every state has established an early hearing detection and intervention (EHDI) program responsible for establishing, maintaining, and improving the system of services needed to serve children with hearing loss and their families. While significant developments have occurred in the last 20 years, challenges to newborn hearing screening, follow up, and early intervention still exist. In 2009, the National Center for Hearing and Assessment Management (NCHAM) initiated a national strategic planning activity to help EHDI program coordinators identify ways to improve their programs through the use of a strengths, weaknesses, opportunities, and threats (SWOT) analysis framework. A SWOT analysis, and subsequent threats, opportunities, weaknesses, and strengths (TOWS) matrix analysis, are commonly used methods of strategic planning. This article summarizes the history and status of EHDI programs to provide a context for the strategic planning process, and explains the methodology used in completing the SWOT analysis.

\section{Introduction}

Approximately 3 per 1,000 newborns in the United States are born with a congenital hearing loss (White, 2007). As shown in Figure 1, permanent hearing loss occurs much more frequently than other conditions for which newborns are routinely screened or for which early detection has proven to be beneficial. Until recently, most children with congenital hearing loss were not identified until they were 2 to 3 years old (Toward Equality, 1988; White, Forsman, Eichwald, \& Muñoz, 2010). According to the U.S. Department

Karl R. White, Ph.D., is a Professor of Psychology and Director of the National Center for Hearing Assessment and Management at Utah State University. Kristina M. Blaiser, Ph.D., CCC-SLP, is an Assistant Professor of Communicative Disorders and Deaf Education at Utah State University. Correspondence concerning this manuscript may be directed to Dr. White at karl.white@usu.edu. 


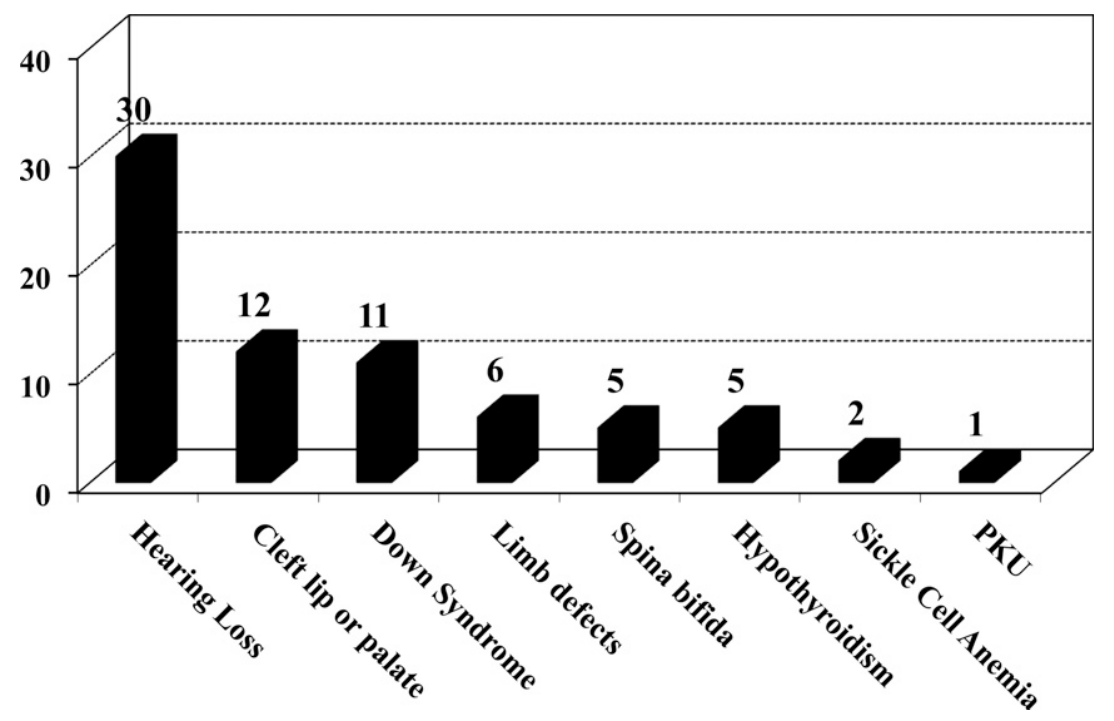

Figure I. Incidence per 10,000 of congenital conditions

of Health and Human Services (DHHS, 1990), late identification means that:

“... It is difficult if not impossible for many [children with congenital hearing loss] to acquire the fundamental language, social and cognitive skills that provide the foundation for later schooling and success in society. When early identification and intervention occur, hearing-impaired children make dramatic progress, are more successful in school, and become more productive members of society" (p. 460).

During the last two decades, newborn hearing screening, diagnosis, and intervention programs-now commonly referred to as early hearing detection and intervention (EHDI) programs - have expanded dramatically. Every state now has an established EHDI program, typically housed within the state's department of health, that is responsible for establishing, maintaining, and improving the system of services necessary to screen newborns for hearing loss; providing timely and appropriate diagnostic assessments for those who do not pass the screen; enrolling children identified with permanent hearing loss in appropriate early intervention programs; coordinating these services with the child's primary health care provider (often referred to as a medical home); and delivering appropriate family education and support. As a part of establishing such a system of services, state-based EHDI programs are also expected to maintain tracking and data management systems, provide ongoing program evaluation and quality improvement activities, and develop 
collaborative relationships with other entities that can assist in the delivery of effective services to children with hearing loss and their families.

Although great progress has been made in establishing universal newborn hearing screening programs (i.e., $97 \%$ of all newborns are now screened for hearing loss before leaving the hospital; CDC, 2010a), persistent problems have limited the benefits of newborn hearing screening for many children with hearing loss and their families. Some of these problems include the following: states are unable to document a completed diagnostic audiological evaluation for almost half of all children who do not pass the newborn hearing screen; many children with a confirmed hearing loss are not enrolled in appropriate early intervention programs; most states have not yet developed effective tracking and data management systems; there is a severe shortage of pediatric audiologists; and many primary health care providers are uninformed about the services and hearing technology available to assist children with hearing loss and their families, limiting the ability of families to obtain such services (White, et al., 2010).

To address these problems and gaps, the Maternal and Child Health Bureau (MCHB) and the Centers for Disease Control and Prevention (CDC) have provided funding for the past 10 years to almost all states and territories to expand and improve their EHDI programs; 43 states have passed legislation related to newborn hearing screening and follow up (National Center for Hearing Assessment and Management [NCHAM], 2010a). Additionally, many important professional and advocacy groups, such as the American Academy of Pediatrics (AAP), the March of Dimes, the American Academy of Audiology, and the Alexander Graham Bell Association for the Deaf and Hard of Hearing, have created position papers, hosted conferences, and convened working groups to make recommendations for improving EHDI programs (NCHAM, $2010 \mathrm{~b}$ ). Despite the widespread recognition of the importance of effective newborn hearing screening, diagnosis, and intervention, progress has been gradual and much work remains to be done.

In January 2009, NCHAM initiated a strategic planning activity to help states identify ways to improve their EHDI programs. A well-known and widelyused strategic planning approach known as a strengths, weaknesses, opportunities, and threats, or SWOT, analysis was used. Since being introduced in the early 1980s, almost 600 published articles have reported the results of SWOT analyses used for strategic planning in fields ranging from marketing, manufacturing, and tourism to instructional technology, agriculture, and health care (Ghazinoory, Abdi, \& Azadegan-Mehr, 2011).

Although the specific way in which a SWOT analysis is implemented varies somewhat depending on the situation to which it is applied, the basic principles are relatively simple and straightforward. First, one or more program goals are identified as the focus of the strategic planning activity. Next, participants use the SWOT analysis framework to identify strengths and weaknesses within a program, and opportunities and threats in the program's external environment. Once these variables are identified, a threats, 
opportunities, weaknesses, and strengths, or TOWS, matrix is used to match the external threats and opportunities with internal weaknesses and strengths of the organization or program (Weihrich, 1982, 1998). Using the TOWS matrix enables strategic planners at various levels of the organization to use strengths to take advantage of opportunities (strength-opportunity strategies); to minimize weaknesses by pursuing opportunities (weakness-opportunity strategies); to use strengths to reduce the organization's vulnerability to threats (strength-threat strategies); and to develop plans to prevent weaknesses from making the program susceptible to threats (weakness-threat strategies).

In the remainder of this article, a short history and current status of EHDI programs will be provided to establish a context for why a SWOT analysis was selected to identify ways in which EHDI programs could be improved. The second part of this paper explains the SWOT methodology that was used to complete strategic planning for improving EHDI programs. Subsequent articles in this volume then describe the results of applying this SWOT methodology to the 12 components identified as important to state EHDI programs, as outlined in Table 1.

Table I. The 12 EHDI components included in the SWOT/TOWS analysis

\begin{tabular}{|c|c|}
\hline EHDI Area & Description \\
\hline $\begin{array}{l}\text { 1. Newborn Hearing } \\
\text { Screening }\end{array}$ & $\begin{array}{l}\text { This EHDI area covers all aspects of newborn hearing } \\
\text { screening, including hospital screenings as well as } \\
\text { home births. }\end{array}$ \\
\hline $\begin{array}{l}\text { 2. Audiological } \\
\text { Evaluation }\end{array}$ & $\begin{array}{l}\text { This EHDI area covers all aspects of audiological evalua- } \\
\text { tions and documentation. }\end{array}$ \\
\hline 3. Early Intervention & $\begin{array}{l}\text { This EHDI area covers all aspects of early intervention } \\
\text { including, but not limited to, Part } C \text { and non-Part } C \\
\text { communication options and technology options. }\end{array}$ \\
\hline $\begin{array}{l}\text { 4. Medical Homes/ } \\
\text { Medical } \\
\text { Professionals }\end{array}$ & $\begin{array}{l}\text { This EHDI area includes the medical home and all } \\
\text { medical professionals responsible for the continuity of } \\
\text { care for children who have or are at-risk for } \\
\text { hearing loss. }\end{array}$ \\
\hline $\begin{array}{l}\text { 5. Loss to } \\
\text { Follow-Up }\end{array}$ & $\begin{array}{l}\text { This EHDI area includes all issues related to loss to follow- } \\
\text { up during the EHDI process (screening to identification } \\
\text { to intervention). }\end{array}$ \\
\hline $\begin{array}{l}\text { 6. Family } \\
\text { Support }\end{array}$ & $\begin{array}{l}\text { This EHDI area includes all aspects of family support } \\
\text { services as well as both educational materials and } \\
\text { services that are culturally and linguistically sensitive } \\
\text { as well as readily available. }\end{array}$ \\
\hline
\end{tabular}


Table I. Cont.

\begin{tabular}{|c|c|}
\hline EHDI Area & Description \\
\hline $\begin{array}{l}\text { 7. Periodic Early } \\
\text { Childhood Hearing } \\
\text { Screening }\end{array}$ & $\begin{array}{l}\text { This EHDI area includes all aspects of screening for } \\
\text { hearing loss in children who passed their initial hearing } \\
\text { screening but are at-risk for hearing loss, have an } \\
\text { identified diagnosis that is associated with hearing loss, } \\
\text { or are being screened to find late-onset hearing loss. }\end{array}$ \\
\hline $\begin{array}{l}\text { 8. Professional } \\
\text { Development }\end{array}$ & $\begin{array}{l}\text { This EHDI area represents all education and professional } \\
\text { development for individuals who provide services } \\
\text { related to universal newborn hearing screening, } \\
\text { identification, and intervention as well as administrators } \\
\text { who oversee various aspects of the EHDI program. }\end{array}$ \\
\hline 9. Infrastructure & $\begin{array}{l}\text { This EHDI area encompasses all aspects of implementing } \\
\text { a universal newborn hearing screening program in a } \\
\text { state. This includes, but is not limited to, staffing at the } \\
\text { state level, funding, legislation/regulations, advisory } \\
\text { committee, public awareness, etc. }\end{array}$ \\
\hline $\begin{array}{l}\text { 10. Data System/ } \\
\text { Management }\end{array}$ & $\begin{array}{l}\text { This EHDI area deals with all aspects of collecting, } \\
\text { compiling, and analyzing data needed for follow-up, } \\
\text { reporting, program improvement, etc. }\end{array}$ \\
\hline 11. Quality Enhancement & $\begin{array}{l}\text { This EHDI area deals with how states use data, staff, and } \\
\text { other resources for quality improvement, program } \\
\text { evaluations, and projects. }\end{array}$ \\
\hline 12. Collaboration & $\begin{array}{l}\text { This EHDI area includes not only the formal } \\
\text { collaboration that occurs, but also cooperation and } \\
\text { systems integration within the state systems and the } \\
\text { private/community sectors. These collaborative efforts } \\
\text { are necessary for infants and young children with hearing } \\
\text { loss to receive appropriate audiological, medical, } \\
\text { educational, family support, and other needed services. }\end{array}$ \\
\hline
\end{tabular}

The results of these analyses suggest concrete ways in which the various components of an EHDI system can be improved by using opportunities to maximize strengths and minimize weaknesses, using strengths to reduce threats, and finding ways to reduce the threats to identified weaknesses.

\section{History and Status of EHDI Programs}

Many people have worked to reduce the age at which children with congenital hearing loss are identified since Ewing and Ewing (1944) called attention to the issue almost 70 years ago. For example, following the pioneering work 
of Marion Downs in the early 1960s (Downs \& Sterritt, 1964, 1967), the Joint Committee on Infant Hearing (JCIH) was established in 1969 with the goal of improving early identification of congenital hearing loss (Northern \& Downs, 1974). Because appropriate technology for population-based newborn hearing screening was not available at the time, the JCIH focused their efforts for the next 25 years on those babies that were at high risk for having a hearing loss (approximately 10\%) (JCIH, 2011).

As new hearing screening technologies became available in the late 1980s and early 1990s, increased resources from the federal government were devoted to screening all newborns for hearing loss and reducing the age of identification. In 1988, the congressionally-mandated Commission on Education of the Deaf (Toward Equality, 1988) recommended that the federal government "... assist states in implementing improved screening procedures for each live birth." A short time later, the U.S. Surgeon General, Dr. C. Everett Koop, issued a challenge to reduce the age at which congenital hearing loss was identified:

"The harmful effects of childhood hearing impairment are given little thought by many people because hearing loss is largely an invisible handicap. An infant with a hearing impairment is generally healthy-looking and develops relatively normally during the first year of life. But if a hearing loss goes undetected in that first year, it will interfere tragically with the child's ability to learn to speak, to do well in school, and to contribute productively to society. . . . In 1989, when I was Surgeon General of the United States, I challenged parents, physicians, state agency staff, and researchers to work together to find better ways to identify very young children with hearing impairments. I set a goal that by the year 2000 all children with significant hearing impairments would be identified before 12 months of age. Although it was an ambitious goal, I was optimistic that it would be accomplished" (Koop, 1993).

Many people believed that Dr. Koop was setting a completely unrealistic goal because less than $3 \%$ of all newborns were screened for hearing loss at the time (Bess, 1993), and no large-scale universal newborn hearing screening programs had been implemented. A short time later, as a result of advances in hearing screening technology for auditory brainstem response (ABR) (Herrmann, Thornton, \& Joseph, 1995) and otoacoustic emissions (OAE) (Kemp, 1978), more and more people began believing in the feasibility and benefits of universal newborn hearing screening.

In March 1993, the National Institutes of Health (NIH, 1993) convened a Consensus Development Conference that recommended "all newborns [be screened] for hearing impairment prior to discharge." Unfortunately, the research evidence and clinical experience on which this recommendation was based was quite sparse. Shortly after the NIH recommendations were issued, Bess and Paradise (1994) concluded that "the [NIH] recommendation of 
universal infant screening falls short of being justified on grounds of practicability, effectiveness, cost, and harm-benefit ratio." Two years later, even though they recognized that "congenital hearing loss is a serious health problem associated with developmental delay in speech and language function," the U.S. Preventive Services Task Force (USPSTF, 1996) concluded that "there is little evidence to support the routine universal screening for all neonates."

Although the conclusions of Bess and Paradise and others, who urged caution and a more gradual approach to the implementation of newborn hearing screening, were widely criticized (White \& Maxon, 1995), there was very little research from large, systematically-implemented universal newborn hearing screening programs to support the recommendations of the NIH at that time. Other than the report of the Rhode Island Hearing Assessment Project (White \& Behrens, 1993), research about newborn hearing screening available at that time was based on small samples of infants (primarily from neonatal intensive care units) over a short period of time. The NIH recommendations and the controversy generated by the Bess and Paradise (1994) article stimulated a great deal of activity over the next 5 years. By the late 1990s, an impressive body of research related to the feasibility, costs, and benefits of newborn hearing screening had been reported (Barsky-Firkser \& Sun, 1997; Finitzo, Albright, \& O'Neal, 1998; Mason \& Herrmann, 1998; Maxon, White, Behrens, \& Vohr, 1995; Mehl \& Thomson, 1998; Vohr, Carty, Moore, \& Letourneau, 1998; and White, 1997), and dozens of large-scale universal newborn hearing screening programs had become operational in various states (White, 2003). The growth of newborn hearing screening over this period is shown in Figure 2.

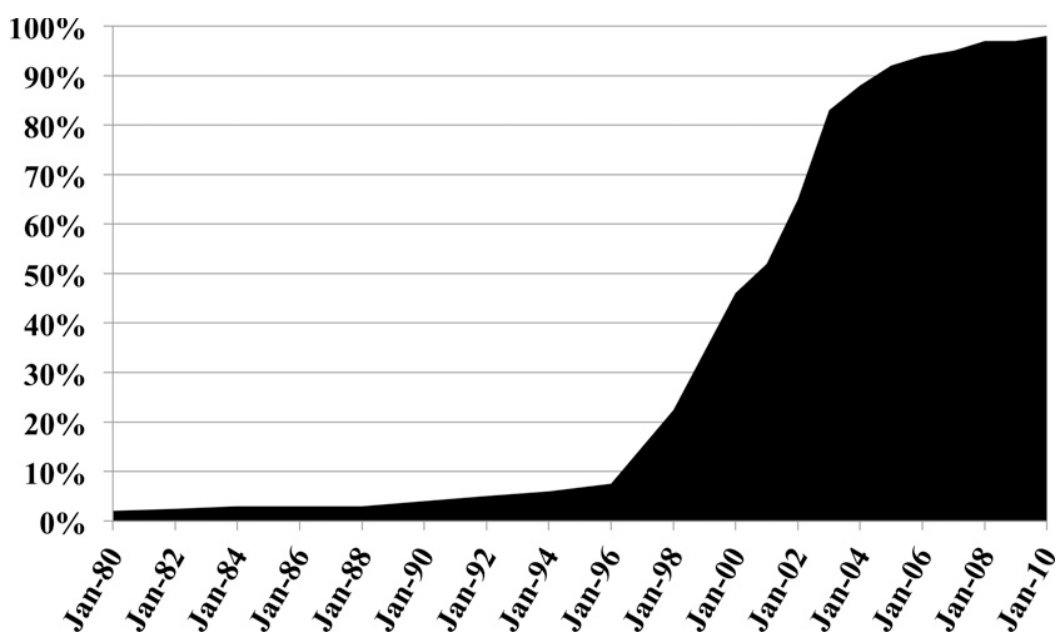

Figure 2. Percentage of newborns screened for hearing loss in the United States from 1980-2010 
The increase in the number of babies being screened for hearing loss led to the realization that screening is only the beginning of a process necessary to identify infants and toddlers with hearing loss and provide them and their families with appropriate services and assistance. There is now widespread agreement that newborn hearing screening programs must be linked to timely and appropriate diagnosis and early intervention programs that are coordinated with the primary health care provider and include appropriate family support (JCIH, 2007). In collaboration with state EHDI program coordinators and other federal, professional, and advocacy organizations, the Centers for Disease Control and Prevention (CDC, 2010b) has promulgated national EHDI goals and program objectives (Table 2).

Table 2. National goals for EHDI programs in the United States (CDC, 2010b)

Goal 1. All newborns will be screened for hearing loss before 1 month of age, preferably before hospital discharge.

Goal 2. All infants who screen positive will have a diagnostic audiological evaluation before 3 months of age.

Goal 3. All infants identified with hearing loss will receive appropriate early intervention services before 6 months of age (medical, audiological, and early intervention).
Hospitals will have a written protocol to ensure all births are screened, results are reported to the infant's parents and primary care physician, and referred infants $(\leq 4 \%)$ receive diagnostic evaluation. Demographic data will be collected for each infant and appropriate educational material provided to parents. States will reduce/eliminate financial barriers to screening and ensure screening of out-of-hospital births.

States will develop audiological diagnostic guidelines and maintain a list of qualified providers to ensure infants referred from screening receive a comprehensive audiological evaluation before 3 months of age and are referred to appropriate services. States will provide appropriate education and/or training about the diagnostic audiological evaluation to parents, primary care physicians, and audiologists.

States will develop policies and resource guides to ensure all parents of children with hearing loss receive appropriate medical (including vision screening and genetic services), audiological, and early intervention services (based on the communication mode chosen by the family). States will ensure that early intervention service providers are educated about issues related to infants and young children with hearing loss. 
Table 2. Cont.

Goal 4. All infants and children with late onset or progressive hearing loss will be identified at the earliest possible time.

Goal 5. All infants with hearing loss will have a medical home as defined by the American Academy of Pediatrics.

Goal 6. Every state will have an EHDI tracking and surveillance system that minimizes loss to follow-up.

Goal 7. Every state will have a system that monitors and evaluates the progress towards the EHDI goals and objectives.
Hospitals and others will report information about risk factors for hearing loss to the state, who will monitor the status of children with risk factors and provide appropriate follow-up services.

A primary care provider who assists the family in obtaining appropriate services will be identified for all infants with confirmed hearing loss before 3 months of age. The state will provide unbiased education about issues related to hearing loss for parents and medical home providers.

A computerized statewide tracking and reporting system will record information about screening results, risk factors, and follow-up for all births. The system will have appropriate safeguards, be linked to other relevant state data systems, and be accessible to authorized health care providers.

A systematic plan for monitoring and evaluation will be developed and implemented by an advisory committee to regularly collect data and provide feedback to families and ensure that infants and children with hearing loss receive appropriate services.

As shown previously in Figure 2, the goal of screening all newborns for hearing loss before 1 month of age has been substantially achieved. The only significant subgroup with which most EHDI programs are still struggling is the $1-2 \%$ of births that occur outside of hospitals. Although this is a very small percentage of all births, it represents 40,000-80,000 infants per year. When an infant does not pass the newborn hearing screen, audiological diagnosis should be completed as soon as possible but definitely no later than 3 months of age. A few states have been able to achieve this goal (White, et al., 2010), but most are still struggling. For example, the CDC reported in 2007 that states could not document that diagnostic evaluations were completed for the $46 \%$ of the infants who needed them (CDC, 2010c). A national evaluation by Mathematica Policy Research, Inc., in 2006, reported that a severe lack of qualified audiologists to provide diagnostic evaluations, a lack of appropriate equipment, and a lack of knowledge among health care providers about the importance and urgency of follow-up testing were factors contributing to the 
poor follow-up rates of diagnostic evaluations (Shulman, Beculides, Saltzman, Ireys, White, \& Forsman, 2010).

Ensuring that children identified with permanent hearing loss receive appropriate educational, audiological, and medical services is also a challenge. For example, JCIH (2007) recommends that all infants with a confirmed hearing loss be referred to a geneticist, an ophthalmologist, and an otolaryngologist who has knowledge of pediatric hearing loss. However, when a national sample of almost 2000 primary health care providers were asked to whom they would refer a newborn patient who had been "diagnosed with a moderate to profound bilateral hearing loss...[when] no other indications are present," only $0.6 \%$ said they would refer to an ophthalmologist, $8.9 \%$ to a geneticist, and $75.6 \%$ to an otolaryngologist. When asked at what age an infant could be fit with hearing aids, almost half said 6 months or older (Moeller, White, \& Shisler, 2006). Clearly, there has been a gap in knowledge of primary health care providers on the appropriate treatment for infants and young children with hearing loss.

Most state EHDI program coordinators report that appropriate educational intervention programs for infants and toddlers with hearing loss are not as widely available as needed (NCHAM, 2010c). Of greater concern, the CDC (2010c) reported that in 2007, state EHDI coordinators were only able to document that $60.8 \%$ of infants and toddlers with permanent hearing loss were enrolled in appropriate early intervention programs, and only about $60 \%$ of those could be documented as having been enrolled before 6 months of age.

The AAP (2002) states that all children should have access to health care that is accessible, family-centered, comprehensive, continuous, coordinated, compassionate, and culturally competent. Such care is referred to as a medical home. According to MCHB (2010), only 57.5\% of all infants were connected with a medical home in 2008. In a 2004 survey, the name of the primary health care provider who would care for the infant during the first three months of life was known for only about $75 \%$ of newborns discharged from the hospital (NCHAM, 2010c). As noted above, many primary care physicians are not well-informed about issues related to early identification of hearing loss.

The CDC (2010d) currently awards funding to 53 states and territories to assist with the development, implementation, and operation of tracking and data management systems for EHDI programs. Although programs are improving, as illustrated above, and the USPSTF now recommends newborn hearing screening (USPSTF, 2008), much remains to be done. A 2006 national survey showed that almost half of all states still depend on mailed or faxed paper forms as a significant part of their data management system (Shulman, Katz, Ireys, \& Besculides, 2006), and fewer than one-third of the states report linkages with other critical programs, such as dried bloodspot screening programs, vital statistics, immunization registries, or early intervention programs (NCHAM, 2010c). 
The overall status of EHDI programs in the United States was summarized recently as follows:

\begin{abstract}
"The current status of EHDI programs in the United States is like the proverbial glass that is half full or half empty. Certainly, the likelihood for an infant or toddler with permanent hearing loss to receive timely and appropriate services is better than ever. ... In contrast to the early 1990s, there is now a solid research and an experiential base for addressing all of these issues, but it will continue to require the commitment and resources of state health officials, hospital administrators, health care providers, and parents. The issues that need to be resolved are complex and will require stakeholders to continue working together over a sustained period" (White, et al., 2010, p. 177).
\end{abstract}

\title{
Strategic Planning for EHDI Programs
}

Most organizations undertake planning in some way, but the degree of sophistication and how systematically this planning is done vary considerably. Conceptually, strategic planning is deceptively simple: define and agree on the organization's goals, analyze the current situation (including resources and obstacles), predict future trends and activities, and then define activities and strategies for achieving those goals. In reality, this is a very complex process and is best achieved when a systematic approach is used for identifying and analyzing factors, both internal and external to the organization, which can be used to achieve the identified goals.

SWOT analysis was developed by the business community in the early 1960s to facilitate strategic planning (Panagiotou, 2003). It provides a systematic framework that is easy to understand and implement for developing a strategic plan for improvement by identifying an organization's intrinsic strengths and weaknesses and linking those to opportunities and threats in the external environment.

SWOT analysis has been used frequently in health care settings, and one of the earliest published reports of a SWOT analysis was done with the administration of nursing programs (Lanzotti, 1991). Examples of how SWOT analyses have been used in the health care field include financing of health care systems (Christiansen, 2002); reducing influenza vaccine shortages (UscherPines, Barnett, Sapsin, Bishai, \& Balicer, 2008); planning postdischarge services for very preterm babies (Pritchard, Colditz, \& Beller, 2008); making better use of information technology in health care (Helms, Moore, \& Ahmadi, 2009); using virtual-reality technology in rehabilitation programs (Rizzo \& Kim, 2005); making geriatric-palliative care more responsive to the needs of elderly patients (Tomasovi, 2005); enhancing medical education (Gordon, et al., 2000; Crow, Hartman, Mahesh, McLendon, Henson, \& Jacques, 2008); increasing participation in smallpox re-vaccination programs (Huerta, 
Balicer, \& Levinthal, 2003); improving breast-feeding education programs (Lee \& Huang, 2005); and expanding and enhancing the effectiveness of tobacco control action plans (Edwards, Brown, Hodgson, Kyle, Reed, \& Wallace, 1999). In fact, in a recent review of over 500 published articles in which SWOT analyses were used, health care applications were the focus of $12.6 \%$ of the articles-more than any other of the 22 different fields in which SWOT had been applied, except for agriculture (Ghazinoory, et al., 2011).

Generating data for a SWOT analysis is straight-forward. Key participants in the program or organization are identified and asked to list strengths, weaknesses, opportunities, and threats related to a specific goal of the organization. This organized approach to generating ideas helps reveal insights that might not otherwise be apparent. Kotter (2006) argues that involving many different people in the organization increases the likelihood that the SWOT analysis will be successful. As noted by Camden, Swaine, Tetreault, and Bergeron (2009):

"An analysis can be completed solely by a programme manager, but when employed with multiple stakeholders, a SWOT analysis is well suited for participatory evaluation because it is based on actors' perceptions and can contribute to participants' awareness and empowerment, facilitating the development of commonly shared organizational goals."

Whatever technique is used to generate ideas about strengths, weaknesses, opportunities, and threats (surveys, interviews, focus groups, etc.), a large amount of information is usually generated. The most frequently used technique for refining these ideas and organizing them into a strategic plan for the organization was first proposed by Weihrich (1982) and is referred to as the TOWS matrix. Although some people have used the term SWOT and TOWS interchangeably, Weihrich argued that the TOWS matrix is a distinct tool that captures data from the SWOT analysis and uses that information to generate specific activities for a strategic plan to accomplish the organization's goals. After a list of strengths, weaknesses, opportunities, and threats is generated, those factors are combined in a two-by-two matrix so that strengths are matched with opportunities, strengths with threats, weaknesses with opportunities, and weaknesses with threats, as shown in Table 3.

Linking strengths, weaknesses, opportunities, and threats in this way makes it easier to identify specific recommendations for actions. Strengthopportunity strategies can be used to identify and pursue opportunities that will most likely strengthen the program. Weakness-opportunity strategies help focus ideas on how to overcome weaknesses necessary to pursue good opportunities. Strength-threat strategies identify activities in which strengths can be used to reduce vulnerability to threats. And weakness-threat strategies can be used to create a defensive plan to prevent weaknesses from making the program more susceptible to threats. Taken together, these activities become the focus of the organization's strategic plan. 
Table 3. The TOWS analysis matrix

\begin{tabular}{|c|c|c|c|}
\hline & \multicolumn{2}{|c|}{ Internal } \\
\hline & & Strengths & Weaknesses \\
\hline \multirow{5}{*}{ 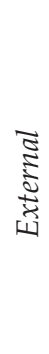 } & $\stackrel{\infty}{2}$ & S-O Strategies & W-O Strategies \\
\hline & $\stackrel{\vec{\Xi}}{\Xi}$ & Pursue opportunities that are a & Overcome weaknesses to pursue \\
\hline & $\frac{9}{7}$ & good fit with strengths. & opportunities. \\
\hline & & S-T Strategies & W-T Strategies \\
\hline & $\frac{\vec{\Xi}}{\stackrel{\vec{E}}{\Xi}}$ & $\begin{array}{l}\text { Identify ways to use strengths to } \\
\text { reduce vulnerability to threats. }\end{array}$ & $\begin{array}{l}\text { Establish defensive plan to prevent } \\
\text { weaknesses from making program } \\
\text { susceptible to threats. }\end{array}$ \\
\hline
\end{tabular}

$\mathrm{S}=$ Strength $\mathrm{W}=$ Weakness $\mathrm{O}=$ Opportunity $\mathrm{T}=$ Threat

\section{Methods for Completing a SWOT Analysis of EHDI Programs}

NCHAM serves as the National Technical Resource Center for all statebased EHDI programs. In this capacity, NCHAM staff work with state EHDI coordinators and other government, professional, and advocacy organizations to improve all aspects of EHDI programs. In late 2008, NCHAM initiated an activity to assist state EHDI coordinators in developing strategic plans for improving their EHDI programs. An online survey was developed by NCHAM staff in which all EHDI coordinators were asked to identify internal strengths and weaknesses and external opportunities and threats to all components of the EHDI program, as shown in Table 1. Prior to completing the survey, EHDI coordinators were provided with background information about SWOT analysis and how to define strengths, weaknesses, opportunities, and threats. A link to the online survey (see Appendix A) was sent to EHDI coordinators, and they were asked to complete the survey by the end of February 2009. All EHDI coordinators were sent a gift card from a national coffee chain as a small incentive for them to complete the questionnaire.

In the survey, coordinators were asked to consider the questions in Table 4 to help them identify strengths, weaknesses, opportunities, and threats for each of the 12 EHDI components. EHDI coordinators were instructed to identify at least one strength, weakness, opportunity, and threat for each of the 12 areas. Many completed more than one, as shown in the results described in subsequent articles in this volume. If a state EHDI coordinator did not know or had no comments about a particular area, they were instructed to type "no comment" or "I don't know."

Forty-nine of the 51 coordinators asked to complete a survey completed at least part of the survey; 47 completed the survey in its entirety. All of the responses 
Table 4. Questions to assist in identifying strengths, weaknesses, opportunities, and threats for EHDI programs

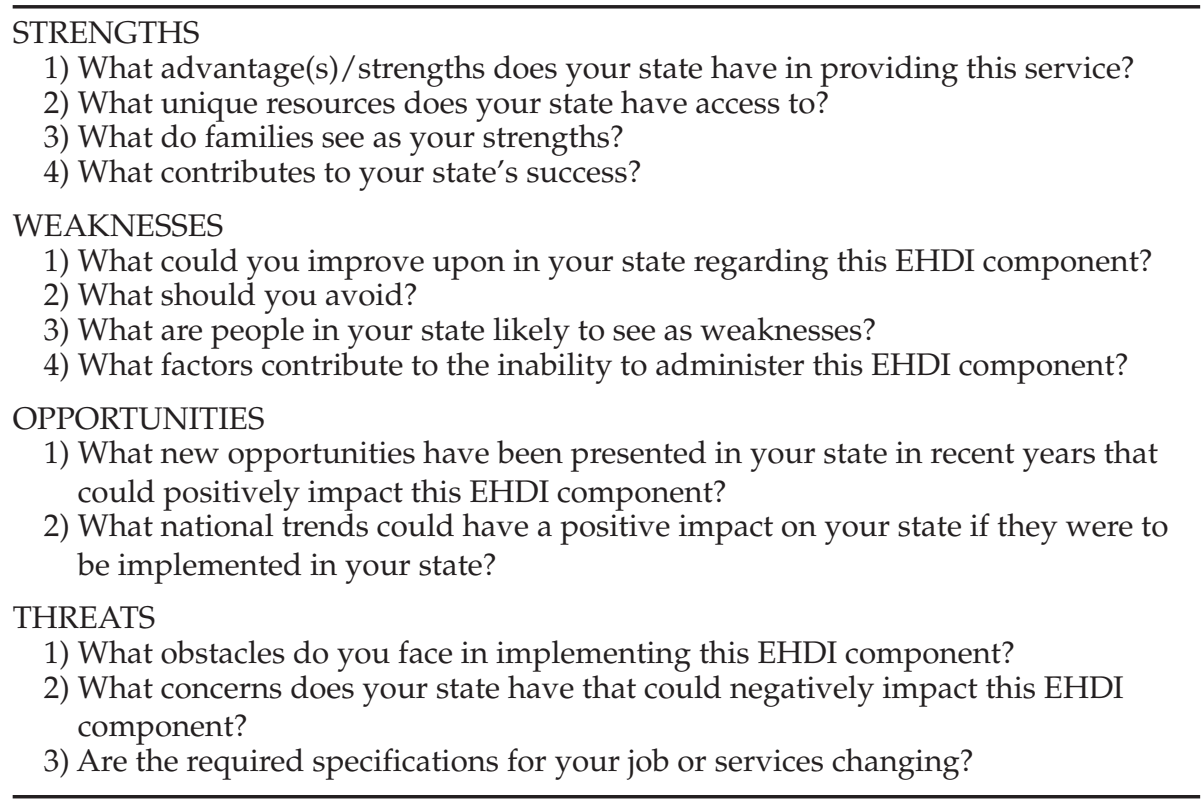

for strengths, weaknesses, opportunities, and threats were listed for each of the 12 areas. A panel of 6 EHDI experts, including an audiologist, speech-language pathologist, teacher of the deaf, state agency coordinator, and an adult with congenital hearing loss, were identified by NCHAM to review the data generated by the state EHDI coordinators, combine items that addressed the same issue but used slightly different wording, tabulate the frequency with which various strengths, weaknesses, opportunities, and threats were identified, and use these data in a TOWS matrix to identify strength-opportunity strategies, weaknessopportunity strategies, strength-threat strategies, and weakness-threat strategies for EHDI programs. Of course some of the strategies are more applicable for state programs and others are more appropriate for national implementation by groups such as MCHB, CDC, AAP, or NCHAM. These strategies are summarized in the remaining papers in this volume with respect to each of the 12 EHDI components listed in Table 1, and indicate the most frequently occurring strategies identified through the SWOT and TOWS matrix analysis. Strategies most appropriate for an individual state varies, but this approach provides a useful national perspective.

\section{Conclusions}

As the following articles in this volume will show, applying SWOT methodology to EHDI programs yielded a number of specific strategies that can be 
used in the ongoing effort to improve services to infants and young children with hearing loss and their families. Of course, SWOT analysis is only one way of generating ideas for how to improve EHDI programs, is a subjective tool, and depends on the perspectives, experiences, and biases of those who participate. In this case, the informants were state EHDI coordinators who are responsible for the overall implementation of EHDI programs in their state and have important information to share about how to improve EHDI programs. Although EHDI coordinators are a logical group because of their extensive experience and day-to-day responsibilities for the operation of EHDI programs, there are other stakeholders that also have important perspectives, such as parents, health care providers, and policy makers. By using the SWOT analysis with input from state EHDI coordinators, a beginning point is established that can be useful for these other groups in suggesting additional or alternative strategic plans.

It is also important to recognize that depending on one's viewpoint, many of the factors identified in this analysis could be a strength, weakness, opportunity, or threat (Gibis, et al., 2001). For example, a data management and tracking system might be a strength in a state that has a well-developed and effective system that is meeting their needs, a weakness in a state that has a system that functions poorly, an opportunity in a state that is considering the acquisition of a system that other states have found valuable, and a threat in a state which has been told it must begin using an existing system that doesn't meet their EHDI-specific needs.

In reading the rest of the articles in this volume, it is important to remember that the combination of data across states provides a national perspective and recommendations for strategic actions that may not be applicable to some states. The SWOT analysis methodology is just as applicable for individual states, but may lead to very different conclusions than the national perspective taken in each of these articles.

It is also important to note that using a TOWS matrix to analyze the results of the items generated by the SWOT analysis is just one way of developing a strategic plan. Using a TOWS matrix after the completion of a SWOT analysis could limit the possible strategies by focusing too narrowly on the pairs of strengthopportunity, strength-threat, weakness-opportunity, and weakness-threat. Again, the results of this approach are offered as a beginning point, but not necessarily the final word in identifying strategic plans to improve EHDI programs.

Finally, this analysis represents information for a single point in time. Ongoing analysis and strategic planning are necessary as EHDI programs continue to evolve, as national policies concerning health care and delivery of services change, and as strategic plans are implemented and change what should be regarded as strengths, weaknesses, opportunities, and threats.

EHDI programs are dynamic. As shown in the subsequent articles in this volume, SWOT analysis has identified specific strategies in all 12 components of an EHDI program. These strategies should be carefully considered by both national and state level EHDI stakeholders to continue 
improvements to state EHDI programs so that children with hearing loss and their families benefit from the advances made in recent years and the opportunities now available.

\section{References}

American Academy of Pediatrics (AAP). (2002). The medical home policy statement. Pediatrics, 110(1), 184-186.

Barsky-Firkser, L., \& Sun, S. (1997). Universal newborn hearing screenings: A three-year experience. Pediatrics, 99(6), E4.

Bess, F. H., (1993). Early identification of hearing loss: A review of the whys, hows, and whens. The Hearing Journal, 46(6), 22-25.

Bess, F. H., \& Paradise, J. L. (1994). Universal screening for infant hearing impairment: Not simple, not risk-free, not necessarily beneficial, and not presently justified. Pediatrics, 93(2), 330-334.

Camden, C., Swaine, B., Tetreault, S., \& Bergeron, S. (2009). SWOT analysis of a pediatric rehabilitation programme: A participatory evaluation fostering quality improvement. Disability and Rehabilitation, 31(16), 1373-1381.

Centers for Disease Control and Prevention (CDC). (2010a). Identifying infants with hearing loss-United States, 1999-2007. Morbidity and Mortality Weekly Report, 59(8), 220-223.

Centers for Disease Control and Prevention (CDC). (2010b). Early hearing detection and intervention (EHDI) national goals. Retrieved May 24, 2011, from http://www.cdc.gov/ncbddd/hearingloss/ehdi-goals.html.

Centers for Disease Control and Prevention (CDC). (2010c). Annual data: Early hearing detection and intervention (EHDI) program. Retrieved May 24, 2011, from http://www.cdc.gov/ncbddd/hearingloss/ehdi-data.html.

Centers for Disease Control and Prevention (CDC). (2010d). Information about early hearing detection and intervention (EHDI) state programs. Retrieved May 24, 2011, from http:/ / www.cdc.gov/ncbddd/hearingloss/ehdi-programs.html.

Christiansen ,T. (2002). A SWOT analysis of the organization and financing of the Danish health care system. Health Policy, 59(2), 99-106.

Crow, S. M., Hartman, S. J., Mahesh, S., McLendon, C. I., Henson, S. W., \& Jacques, P. (2008). Strategic analyses in nursing schools: Attracting, educating, and graduating more nursing students. The Health Care Manager, 27(3), 234-244.

Downs, M. P., \& Sterritt, G. M. (1964). Identification audiometry for infants: A preliminary report. Journal of Audiological Research, 4, 69-80.

Downs, M. P., \& Sterritt, G. M. (1967). A guide to newborn and infant hearing screening programs. Archives of Otolaryngology, 85, 37-44.

Edwards, R., Brown, J., Hodgson, P., Kyle, D., Reed, D., \& Wallace, B. (1999). An action plan for tobacco control at the regional level. Public Health, 113, 165-70.

Ewing, I. R., \& Ewing, A. W. G. (1944). The ascertainment of deafness in infancy and early childhood. The Journal of Laryngology and Otology, 59, 309-333. 
Finitzo, T., Albright, K., \& O'Neal, J. (1998). The newborn with hearing loss: Detection in the nursery. Pediatrics, 102(6), 1452-1460.

Ghazinoory, S., Abdi, M., \& Azadegan-Mehr, M. (2011). SWOT methodology: A state-of-the-art review for the past, a framework for the future. Journal of Business Economics and Management, 12(1), 24-48.

Gibis, B., Artiles, J., Corabian, P., Meiesaar, K., Koppel, A., Jacobs, P., et al. (2001). Application of strengths, weaknesses, opportunities and threats analysis in the development of a health technology assessment program. Health Policy, 58, 27-35.

Gordon, J., Hazlett, C., Ten-Cate, O., Mann, K., Kilminister, S., Prince, K., et al. (2000). Strategic planning in medical education: Enhancing the learning environment for students in clinical settings. Medical Education, 34, 841-850.

Helms, M. M., Moore, R., \& Ahmadi, M. (2009). Information technology (IT) and the healthcare industry: A SWOT analysis. In J. Tan (Ed.), Medical informatics: Concepts, methodologies, tools, and applications (pp. 134-152). Hamilton, Ontario: McMaster University.

Herrmann, B. S., Thornton, A. R., \& Joseph, J. M. (1995). Automated infant hearing screening using the ABR: Development and validation. American Journal of Audiology, 4(2), 6-14.

Huerta, M., Balicer, R., \& Leventhal, A. (2003). SWOT analysis: Strengths, weaknesses, opportunities and threats of the Israeli smallpox revaccination program. The Israel Medical Association Journal, 5, 42-46.

Joint Committee on Infant Hearing (JCIH). (2007). Position statement: Principles and guidelines for early hearing detection and interventions programs. Pediatrics, 120, 898-921.

Joint Committee on Infant Hearing (JCIH). (2011). Position statements from the Joint Committee on Infant Hearing. Retrieved May 25, 2011, from http:/ / www. jcih.org/posstatemts.htm.

Kemp, D. T. (1978). Stimulated acoustic emissions from the human auditory system. Journal of the Acoustical Society of America, 89, 2027-2067.

Koop, C. E. (1993). We can identify children with hearing impairment before their first birthday. Seminars in Hearing 14(1), Foreword.

Kotter, J. P. (2006). Leading change. Boston: Harvard University Press.

Lanzotti, L. M. (1991). Staff participation in a strength, weakness, opportunity, and threats analysis. Journal of Nursing Administration, 21, 67-69.

Lee, P., \& Huang, C. (2005). Using SWOT to analyze breastfeeding education results in a medical center. $\mathrm{Hu} \mathrm{Li} \mathrm{Za}$ Zhi (Chinese Journal of Nursing), 52, $77-82$.

Mason, J. A., \& Herrmann, K. R. (1998). Universal infant hearing screening by automated auditory brainstem response measurement. Pediatrics, 101(2), 221-228.

Maternal and Child Health Bureau (MCHB). (2010). National survey of children's health. Retrieved May 24, 2011, from http://nschdata.org/ DataQuery/DataQueryResults.aspx. 
Maxon, A. B., White, K. R., Behrens, T. R., \& Vohr, B. R. (1995). Referral rates and cost efficiency in a universal newborn hearing screening program using transient evoked otoacoustic emissions (TEOAE). Journal of the American Academy of Audiology, 6, 271-277.

Mehl, A. L., \& Thomson, V. (1998). Newborn hearing screening: The great omission. Pediatrics, 101(1), 1-6.

Moeller, M. P., White, K. R., \& Shisler, L. (2006). Primary care physicians' knowledge, attitudes, and practices related to newborn hearing screening. Pediatrics, 118(4), 1357-1370.

National Center for Hearing Assessment and Management (NCHAM) (2010a). State EHDI/UNHS mandates: Summary table. Retrieved May 24, 2011, from http://www.infanthearing.org/legislative/summary/index. html.

National Center for Hearing Assessment and Management (NCHAM). (2010b). Position statements. Retrieved May 24, 2011, from http://www. infanthearing.org/positionstatements/index.html

National Center for Hearing Assessment and Management (NCHAM). (2010c). 2004 state EHDI survey. Retrieved May 24, 2011, from http:/ /www. infanthearing.org/survey/2004statesurvey/index.html.

National Institutes of Health (NIH). (1993). Early identification of hearing impairment in infants and younger children. Rockville, MD: National Institutes of Health.

Northern, J. L., \& Downs, M. P. (1974). Hearing in children. Baltimore, MD: Williams and Wilkins.

Panagiotou, G. (2003). Bringing SWOT into focus. Business Strategy Review, 14(2), 8-10.

Pritchard, M. A., Colditz, P. B., \& Beller, E. M. (2008). Parental experiences and preferences which influence subsequent use of post-discharge health services for children born very preterm. Journal of Paediatrics and Child Health, $44,281-284$.

Rizzo, A., \& Kim, G. J. (2005). A SWOT analysis of the field of virtual reality rehabilitation and therapy. Presence, 14(2), 119-146.

Shulman, S., Katz, A., Ireys, H., \& Besculides, M. (2006). Evaluation of HRSA's universal newborn hearing screening and intervention program: Final report. Cambridge, MA. Mathematics Policy Research, Inc.

Shulman, S., Besculides, M., Saltzman, A., Ireys, H., White, K. R., \& Forsman, I. (2010). Evaluation of the universal newborn hearing screening and intervention program. Pediatrics, 126, 19-27.

Tomasovi, N. (2005). Geriatric-palliative care units model for improvement of elderly care. Collegium Antropologicum, 29, 277-282.

Toward Equality. (1988). A report to the Congress of the United States: Toward equality - Commission on Education of the Deaf. Washington, DC: U.S. Government Printing Office. 
U.S. Department of Health and Human Services (DHHS). (1990). Healthy People 2000: National health promotion and disease prevention objectives. Washington, DC: Public Health Service.

U.S. Preventive Services Task Force (USPSTF). (1996). Screening for hearing impairment. In U.S. Preventive Services Task Force Guide to Clinical Preventive Services, 2nd Ed. (pp. 393-405). Baltimore, MD: Williams \& Wilkins.

U.S. Preventive Services Task Force (USPSTF). (2008). Universal screening for hearing loss in newborns: U.S. Preventive Services Task Force recommendation statement. Pediatric, 122(1), 143-148.

Uscher-Pines, L., Barnett, D. J., Sapsin, J. W., Bishai, D. M., \& Balicer, R. D. (2008). A systematic analysis of influenza vaccine shortage policies. Public Health, 122, 183-191.

Vohr, B. R., Carty, L. M., Moore, P. E., \& Letourneau, K. (1998). The Rhode Island Hearing Assessment Program: Experience with statewide hearing screening (1993-1996). Journal of Pediatrics, 133, 353-357.

Weihrich, H. (1982). The TOWS matrix - a tool for situational analysis. Long Range Plan, 15, 54-66.

Weihrich, H. (1998). Daimler-Benz's move towards the next century with the TOWS matrix. In Dyson, R., \& O'Brien, F. (Eds.), Strategic development: methods and models (pp. 69-79). Indianapolis, IN: Wiley.

White, K. R. (1997). Issues \& evidence: Prevalence of congenital hearing loss. Retrieved May 24, 2011, from http://www.infanthearing.org/summary/ prevalence.html.

White, K. R. (2003). The current status of EHDI programs in the United States. Mental Retardation and Developmental Disabilities Research Review, 9(2), 79-88.

White, K. R. (2007). Early intervention for children with permanent hearing loss: Finishing the EHDI revolution. The Volta Review, 106(3), 237-258.

White, K. R., \& Behrens, T. R. (Eds.). (1993). The Rhode Island Hearing Assessment Project: Implications for universal newborn hearing screening. Seminars in Hearing, 14(1).

White, K. R., Forsman, I., Eichwald, J., \& Muñoz, K. (2010). The evolution of early hearing detection and intervention programs in the United States. Seminars in Perinatology, 34(2), 170-179.

White, K. R., \& Maxon, A. B. (1995). Universal screening for infant hearing impairment: Simple, beneficial, and presently justified. International Journal of Pediatric Otorhinolaryngology, 32, 201-211. 


\section{Appendix A: Online Survey Used in SWOT Analysis of State EHDI Programs}

\section{NCHAM Survey of EHDI Components}

\section{Introduction}

We are looking forward to your participation in the upcoming National EHDI Meeting in Dallas, Texas. To help prepare for the State Team Meetings that will occur on Monday and Tuesday, as well as the EHDI coordinator meeting that Irene Forsman has requested on Wednesday (March 11th), we would like you to complete the following survey. We know many of you are completing the CDC report that is due on February 12th, so we are requesting that this be completed no later than February 20th. The survey has been designed to gather information about internal strengths and weaknesses and external opportunities and threats related to 12 EHDI components that each state faces each day while trying to implement an effective and efficient Early Hearing Detection and Intervention system. Your participation in this survey is very important to us. This survey will take approximately 30 minutes to complete. THANK YOU FOR YOUR HELP!!!

\section{Demographics}

\section{* 1. Please enter your demographic information below:}

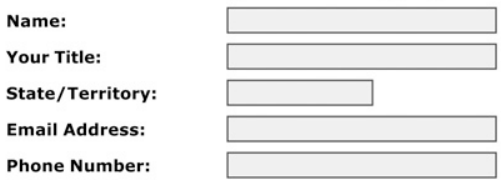

Phone Number:

\section{Please indicate the range which your state reported as the occurrent} birth rate in recent years.
a. $1-25,000$
b. $25,001-50,000$
c. $50,001-100,000$
d. $100,001-150,000$
e. $150,001-200,000$
f. $200,000-250,000$
g. 250,001 or more
Other (please specify)

3. How long has your state been providing state-wide universal newborn hearing screening (volunteer and/or mandated)? 
NCHAM Survey of EHDI Components

\section{If you completed a State Action Plan from the February 2008 EHDI Conference in New Orleans, please answer the following questions:}

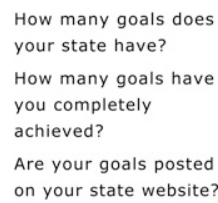

\section{If you completed a State Family Support Plan from the Investing in Family Support Conference in October of 2008 in Raleigh, NC, please answer the following questions:}

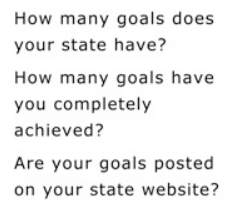

\section{SWOT Questions}

In the next section of the survey you will be asked to report on internal strengths and weakness as well as external opportunities and threats for each EHDI component that is typically included in a statewide EHDI program. Please identify at least one strength, weakness, opportunity and threat for each component. The EHDI component is posted at the top of each page. If you do not have any comments or don't know, then type "no comment" or "don't know".

Each component will be listed on a separate page in what follows. To assist you with identifying strengths, weaknesses, opportunities and threats, as you provide information about each component in your state's EHDI program, please refer to these questions:

\section{STRENGTHS}

1) What advantage(s)/strengths does your state have in providing this service?

2) What unique resources does your state have access to?

3) What do families see as your strengths?

4) What contributes to your state's success?

\section{WEAKNESSES}

1) What could you improve upon in your state regarding this EHDI component?

2) What should you avoid?

3) What are people in your state likely to see as weaknesses?

4) What factors contribute to the inability to administer this EHDI component?

\section{OPPORTUNITIES}

1) What new opportunities have been presented in your state in recent years that could positively impact this EHDI component?

2) What national trends could have a positive impact on your state if they were to be implemented in your state? 


\section{NCHAM Survey of EHDI Components}

THREATS

1) What obstacles do you face in implementing this EHDI component?

2) What concerns do your state have that could negatively impact?

3) Are the required specifications for your job or services changing?

YOU MAY WANT TO PRINT THIS PAGE TO ASSIST YOU WITH THE FOLLOWING PAGES.

\section{Newborn Hearing Screening}

EHDI Component 1: Newborn Hearing Screening

This EHDI component covers all aspect newborn hearing screening, including hospital screenings as well as home births.

* 6. For EHDI component "Newborn Hearing Screening", please identify at least one for each of the following as it pertains to your state EHDI program:

Strengths

Weaknesses

Opportunities

Threats

\section{Audiological Evaluation}

EHDI Component 2: Audiological Evaluation

This EHDI component covers all aspect of audiological evaluations and documentation.

* 7. For EHDI component "Audiological Evaluation", please identify at least one for each of the following as it pertains to your state EHDI program:

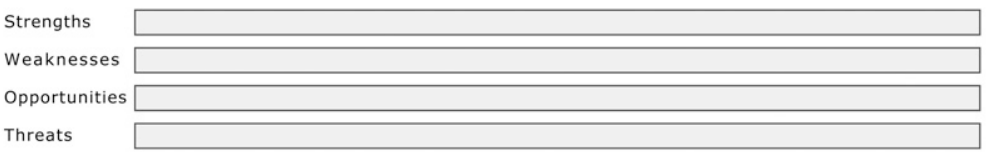

\section{Early Intervention}

EHDI Component 3: Early Intervention

This EHDI component covers all aspects of early intervention including but not limited to Part C, non Part-C, communication options, and technology options. 


\section{NCHAM Survey of EHDI Components}

* 8. For EHDI component "Early Identification", please identify at least one for each of the following as it pertains to your state EHDI program:

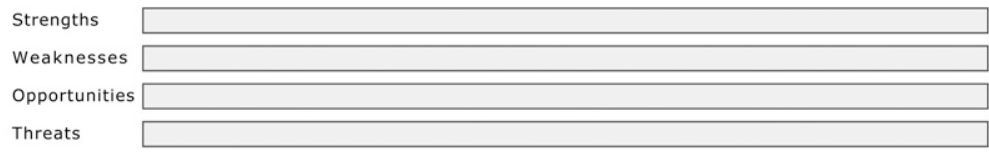

\section{Medical Homes/Medical Professionals}

EHDI Component 4: Medical Homes/Medical Professionals

For continuity of care for children who present or are at-risk for hearing loss, the medical home and all medical professionals involved in the children's care are essential in the EHDI system.

\section{* 9. For EHDI component "Medical Homes/Medical Professionals", please identify at least one for each of the following as it pertains to your state EHDI program:}

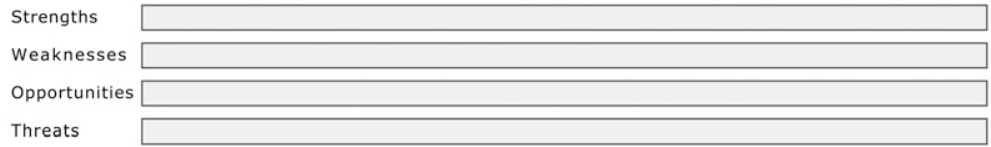

\section{Lost to Follow-Up}

EHDI Component 5: Lost to Follow-Up

This EHDI component includes all issues related to lost to follow-up during the EHDI process (screening to identification to intervention).

\section{* 10. For EHDI component "Lost to Follow-Up", please identify at least one for} each of the following as it pertains to your state EHDI program:

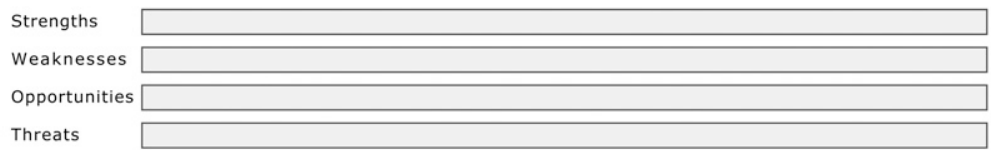

\section{Family Support}

EHDI Component 6: Family Support

Family support is vital to the EHDI system. This component includes all aspects of family support services as well as both educational materials and services that are culturally and linguistically sensitive and readily available. 


\section{NCHAM Survey of EHDI Components}

* 11. For EHDI component "Family Support", please identify at least one for each of the following as it pertains to your state EHDI program:

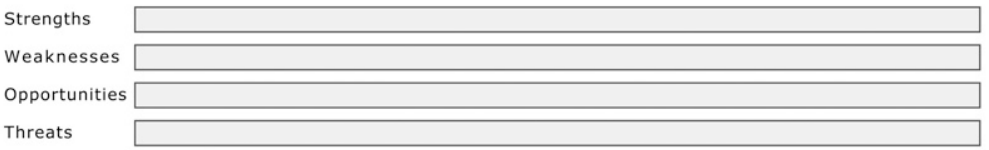

\section{Periodic Early Childhood Hearing Screening}

EHDI Component 7: Periodic Early Childhood Hearing Screening

This component includes all aspects of screening for hearing loss in children who passed their initial hearing screening but have a risk factor for hearing loss, have an identified diagnosis that is associated with hearing loss, or are being screened to find late-onset hearing loss.

\section{* 12. For EHDI component "Periodic Early Childhood Screening", please} identify at least one for each of the following as it pertains to your state EHDI program:

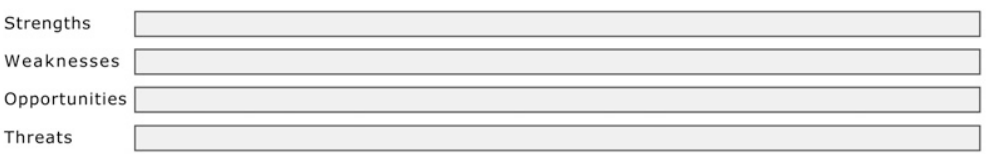

\section{Professional Development}

EHDI Component 8: Professional Development

This EHDI component represents all education and professional development for individuals who provide services related to universal newborn hearing screening, identification, intervention, as well as administrators who oversee various aspects of the EHDI program.

\section{* 13. For EHDI component "Professional Development", please identify at least one for each of the following as it pertains to your state EHDI program:}

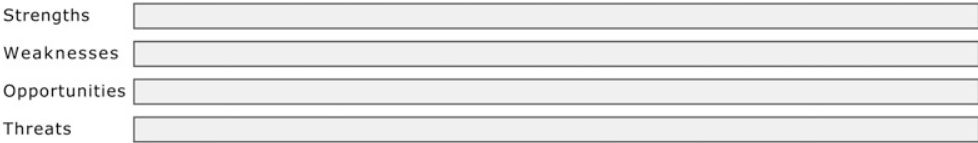

\section{Infrastructure}

EHDI Component 9: Infrastructure 


\section{NCHAM Survey of EHDI Components}

This EHDI component encompasses all aspects of implementing a UNHS program in your state. This includes, but not limited to, staffing at the state level, funding, legislature/regulations, advisory committee, public awareness, etc.

\section{* 14. For EHDI component "Infrastructure", please identify at least one for} each of the following as it pertains to your state EHDI program:

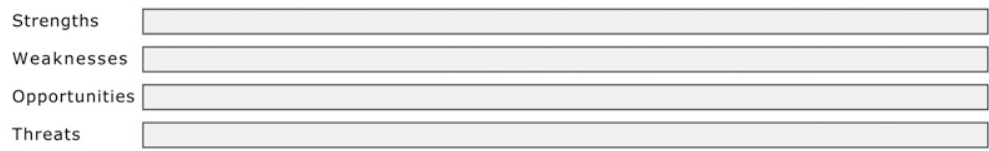

\section{Data System and Management}

EHDI Component 10: Data System and Management

This EHDI component deals with all aspects of collecting, compiling, and analyzing data needed for follow-up, reporting, program improvement, etc.

* 15. For EHDI component "Data Collection and Management", please identify at least one for each of the following as it pertains to your state EHDI program:

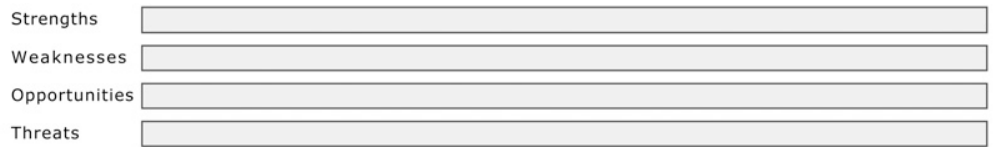

14. Quality Enhancement/Continuous Improvement/Evaluation

EHDI Component 11: Quality Enhancement/Continuous Improvement/Education

This EHDI component deals with how your state uses data, staff, and other resources for quality improvement, program evaluations, and projects.

\section{* 16. For EHDI component "Quality Enhancement/Continuous} Improvement/Evaluation", please identify at least one for each of the following as it pertains to your state EHDI program:

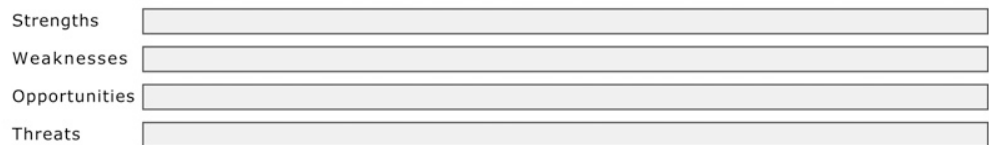

\section{Collaboration}




\section{NCHAM Survey of EHDI Components}

EHDI Component 12: Collaboration

Interagency collaboration is very important for the EHDI programs. This component includes not only the formal collaboration that occurs but also cooperation and systems integration within the state systems and the private/community sectors. These collaborative efforts are necessary for infants and young children with hearing loss to receive appropriate audiological, medical, educational, family support, and other needed services.

\section{* 17. For EHDI component "Collaboration", please identify at least one for each of the following as it pertains to your state EHDI program:}

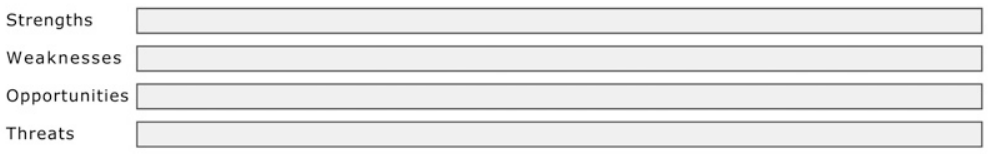

\section{Thank you!}

Thank you very much for taking the time to complete this survey. The summarized information will be used for the State Team Meetings and the EHDI coordinator meeting at the upcoming EHDI conference in Dallas. If you, or members of your state team have not yet registered for the meeting, be sure to do so soon by visiting www.ehdiconference.org. 
The Volta Review, Volume 111(2), Summer 2011, 109-120

\title{
Newborn Hearing Screening: An Analysis of Current Practices
}

\author{
K. Todd Houston, Ph.D., CCC-SLP, LSLS Cert. AVT; \\ Tamala S. Bradham, Ph.D., CCC-A; Karen F. Muñoz, Ed.D., CCC-A; and \\ Gayla Hutsell Guignard, M.A., CCC-A/SLP, LSLS Cert. AVT
}

State coordinators of early hearing detection and intervention (EHDI) programs completed a strengths, weaknesses, opportunities, and threats, or SWOT, analysis that consisted of 12 evaluative areas of EHDI programs. For the newborn hearing screening area, a total of 293 items were listed by 49 EHDI coordinators, and themes were identified within each SWOT category. A threats, opportunities, weaknesses, and strengths, or TOWS, analysis yielded 12 recommendations that focused on similar themes. Out of these 12 recommendations, 3 distinct strategies emerged. States are encouraged to: 1) enhance existing screening practices by requiring systematic data collection, reporting, and automation as well as integration of universal newborn hearing screening database systems with other public health initiatives; 2) leverage existing state legislative support to update and expand current universal newborn hearing screening policies and garner increased resources (i.e., personnel and funding); and 3) reduce loss to follow-up by integrating and centralizing data collection and tracking among various agencies and addressing the screening needs of special populations (e.g., home births, non-native English speaking families, religious and cultural minorities). Addressed appropriately, these recommendations can substantially strengthen newborn hearing screening efforts in state EHDI programs.

K. Todd Houston, Ph.D., CCC-SLP, LSLS Cert. AVT, was an Assistant Professor in the Department of Communicative Disorders and Deaf Education at Utah State University at the time of this study and is currently at The University of Akron. Tamala S. Bradham, Ph.D., CCC-A, is an Assistant Professor in the School of Medicine, Department of Hearing and Speech Sciences, at Vanderbilt University. Karen F. Muñoz, Ed.D., CCC-A, is an Assistant Professor in the Department of Communicative Disorders and Deaf Education at Utah State University. Gayla Hutsell Guignard, M.A., CCC-A/SLP, LSLS Cert. AVT, is the Program Director of Indiana's Early Hearing Detection \& Intervention (EHDI) program in the Department of Genomics \& Newborn Screening at the Indiana State Department of Health. Correspondence concerning this manuscript should be addressed to Dr. Houston at ktodd.houston@gmail.com. 


\section{Introduction}

In the United States, permanent hearing loss is the most common birth defect, affecting approximately 3 newborns per 1,000 births (White, 2004). Since the late 1990s, several studies have demonstrated that diagnosis of hearing loss and intervention delivered early - before the child is 6 months old often leads to age-appropriate communication outcomes by the time the child enters kindergarten or first grade (Calderon, 2000; Calderon \& Naidu, 2000; Moeller, 2000; Yoshinaga-Itano, Sedey, Coulter, \& Mehl, 1998). The past decade has also seen remarkable progress in establishing strong and viable universal newborn hearing screening (UNHS) programs in hospitals and birthing centers in all 50 states, U.S. territories, and the District of Columbia. Local UNHS programs provide the underpinnings of effective state-level EHDI systems, which now follow national standards established by federal agencies (Centers for Disease Control and Prevention [CDC], 2010a). These standards, which outline screening practices, strategies for data collection and tracking, and follow-up procedures, have been adopted widely, resulting in 43 states (plus the District of Columbia and Puerto Rico) now requiring or mandating UNHS and tracking programs (National Center for Hearing and Assessment [NCHAM], 2010). As a result, more than $97 \%$ of all newborns today receive a hearing screening before they leave the hospital or birthing center (CDC, 2010b). Most importantly, because of these efforts, the age of identification of hearing loss has decreased from an average of 2 to 3 years of age to an average of 2 to 3 months of age (White, Forsman, Eichwald, \& Muñoz, 2010).

Although UNHS has proven to be effective, an estimated $50 \%$ of infants referred from UNHS do not receive a timely diagnosis and intervention, or they receive services but are not documented in the tracking system (CDC, 2008). In 2007, the Joint Committee on Infant Hearing (JCIH) released its current position statement $(\mathrm{JCIH}, 2007)$ that addressed various issues related to UNHS in all settings (e.g., well-baby nursery, neonatal intensive care unit (NICU), outpatient screening, home births) and made recommendations that support a more effective and seamless EHDI system to decrease loss to followup and improve outcomes for infants with hearing loss. Considerable efforts are now being employed at the state and national levels to ensure that infants receive a timely diagnosis of hearing loss and are promptly enrolled in early intervention services.

The purpose of this study was to provide a systematic review of UNHS efforts using a strengths, weaknesses, opportunities, and threats, or SWOT, and a threats, opportunities, weaknesses, and strengths, or TOWS, analysis to determine the current status of UNHS programs, and to identify factors that contribute to successful practices as well as those that may reduce overall effectiveness. 


\section{Methods}

Fifty-one state EHDI coordinators were asked to complete an online survey. To examine issues related to newborn hearing screening, the survey asked for the EHDI coordinator to report at least 1 item in the following four areas to assist with strategic planning: strength, weakness, opportunity, and threat. The responses obtained were reviewed by a panel of experts in the field and categorized into common themes in each of these four strategic planning areas. To generate recommendations from the SWOT analysis, a TOWS matrix was used to match identified strengths with opportunities (S-O strategy), strengths with threats (S-T strategy), weaknesses with opportunities (W-O strategy), and weaknesses with threats (W-T strategy). For an in-depth review of the methodology, the reader is referred to White and Blaiser, 2011.

\section{Results}

Forty-nine state EHDI coordinators (96\%) completed the newborn hearing screening question of the SWOT questionnaire. The respondents generated 91 items in the strengths category, 75 items in the weaknesses category, 63 items in the opportunities category, and 64 items in the threats category for a total of 293 responses. Based on these responses, several specific themes were generated for each category: 9 for strengths, 11 for weaknesses, 8 for opportunities, and 7 for threats (Table 1). Each category also had a miscellaneous section, which included no comment, N/A, or items that did not fit within the themes identified.

\section{Strength Characteristics}

The three most frequently cited strengths were screening practices $(34 \%)$, legislation $(20 \%)$, and data collection (14\%) (Table 2). Thirty-four percent of respondents reported that most birthing centers and hospitals in their states had successfully implemented UNHS programs, with a majority of coordinators reporting newborn screening rates between $97-99 \%$. Several respondents reported improved screening rates, increased collaboration among hospitals and birthing centers, and greater cooperation from midwives. Twenty percent of respondents noted strengths associated with passage of state legislation or policy in support of UNHS, requiring hospitals and birthing centers to conduct UNHS programs and thus increasing the rates of newborn hearing screening. Improved methods of data collection and tracking of children who do not pass a hearing screen was also cited as a strength by $14 \%$ of respondents. EHDI coordinators reported increased coordination and integration with other state agencies or tracking systems, such as metabolic blood screening and electronic birth certificate program, as strengths that can significantly improved overall data collection and tracking for UNHS. 
Table I. Frequency of themes within each SWOT category $(\mathrm{n}=293)$

\begin{tabular}{|c|c|c|}
\hline SWOT category & Themes & Frequency n $(\%)$ \\
\hline Strengths $(n=91)$ & $\begin{array}{l}\text { Screening Practice Compliance } \\
\text { Legislation } \\
\text { Data Collection } \\
\text { Follow-Up } \\
\text { EHDI Staff/Operations } \\
\text { Relationships/Communication } \\
\text { Grant Funding } \\
\text { Families } \\
\text { Equipment }\end{array}$ & $\begin{array}{l}31(34 \%) \\
18(20 \%) \\
13(14 \%) \\
8(9 \%) \\
8(9 \%) \\
6(7 \%) \\
3(3 \%) \\
2(2 \%) \\
2(2 \%)\end{array}$ \\
\hline Weaknesses $(\mathrm{n}=75)$ & $\begin{array}{l}\text { Data } \\
\text { Follow-Up Issues } \\
\text { Home Births } \\
\text { Legislation/Regulations } \\
\text { Equipment } \\
\text { Screening Issues } \\
\text { Lack of Providers/Professionals } \\
\text { EHDI Staff/Operations } \\
\text { Cultural/Language Barriers } \\
\text { Funding Issues } \\
\text { Miscellaneous/No Comment }\end{array}$ & $\begin{array}{c}18(24 \%) \\
11(15 \%) \\
9(12 \%) \\
7(9 \%) \\
6(8 \%) \\
6(8 \%) \\
5(7 \%) \\
4(5 \%) \\
4(5 \%) \\
3(4 \%) \\
2(3 \%)\end{array}$ \\
\hline Opportunities $(n=63)$ & $\begin{array}{l}\text { Data } \\
\text { Training/Education Resources } \\
\text { Legislation } \\
\text { Follow-Up Issues } \\
\text { Funding } \\
\text { Relationships } \\
\text { Infrastructure } \\
\text { Miscellaneous/No Comment }\end{array}$ & $\begin{aligned} & 16(25 \%) \\
& 12(19 \%) \\
& 9(14 \%) \\
& 8(13 \%) \\
& 5(8 \%) \\
& 5(8 \%) \\
& 4(6 \%) \\
& 4(6 \%)\end{aligned}$ \\
\hline Threats $(n=64)$ & $\begin{array}{l}\text { Funding } \\
\text { Personnel } \\
\text { Follow-Up } \\
\text { Miscellaneous/No Comments } \\
\text { Data } \\
\text { Legislation } \\
\text { Equipment }\end{array}$ & $\begin{array}{c}27(42 \%) \\
14(22 \%) \\
6(9 \%) \\
6(9 \%) \\
5(8 \%) \\
3(5 \%) \\
3(5 \%)\end{array}$ \\
\hline
\end{tabular}

Other strengths included follow-up practices (9\%), EHDI staff and operations $(9 \%)$, relationships / communication $(7 \%)$, grant funding $(3 \%)$, families $(2 \%)$, and equipment ( $2 \%)$. Related to follow-up practices, EHDI coordinators reported that sending letters, making phone calls, and automatic eligibility for early intervention services were viewed as effective practices. Related to EHDI staff and operations, strengths included dedicated staff, having audiologists on the EHDI staff, and having the means to provide individual case management. Active advisory groups, effective brochures, and regional meetings 
Table 2. Top three themes for strengths

\begin{tabular}{ll}
\hline \multicolumn{1}{c}{ Themes } & \multicolumn{1}{c}{ Descriptions } \\
\hline Screening Practices & All birthing centers \& hospitals screening newborns. \\
& $99 \%$ of children in hospitals receive a screen. \\
& Statewide participation by all birthing centers \& some \\
& midwives. \\
Tegislation & The state has a mandate for UNHS. \\
& Strong legislative \& administrative support. \\
Data Collection & Database has been integrated with newborn metabolic \\
& screening. \\
& Hospitals are reporting their data electronically. \\
& Excellent data integration system.
\end{tabular}

helped with overall communications and relationships. Under grant funding, EHDI coordinators reported that support from federal agencies was helpful and appreciated. Finally, having relevant equipment and family-centered services were also considered strengths.

\section{Weakness Characteristics}

Data collection (24\%), follow-up issues (15\%), and home births (12\%) were the three most commonly identified weaknesses (Table 3). Nearly one-fourth $(24 \%)$ of the respondents reported that data collection and tracking of newborns who did not pass a hearing screen was considered a weakness within their program or state. Coordinators expressed concern over antiquated "pen and paper" systems of reporting data that - for some states - required results to be faxed from the hospital or birthing center and then re-typed into a central, computerized database. Other coordinators reported periodically receiving

Table 3. Top three themes for weaknesses

\begin{tabular}{ll}
\hline \multicolumn{1}{c}{ Themes } & \multicolumn{1}{c}{ Descriptions } \\
\hline Data Collection & $\begin{array}{l}\text { Large amount of data entry. } \\
\text { Too much inconsistency in hospitals' reporting of data. } \\
\text { Manual reporting system. }\end{array}$ \\
Follow-Up Issues & $\begin{array}{l}\text { Inconsistent hospital follow-up with families. } \\
\text { Inconsistent listing of primary care physician. } \\
\text { Home Births }\end{array}$ \\
& Not all hospitals do their own rescreens. \\
& Limited funds and staff to provide screenings in home. \\
& Need to improve outreach to midwives. \\
\hline
\end{tabular}


only aggregate data from hospitals or birthing centers, resulting in inconsistent reporting and incomplete data sets.

Follow-up issues continue to be a challenge for $15 \%$ of EHDI UNHS programs, including "out-of-hospital" births, inconsistent information shared with families, and inadequate notification of the infant's primary care physician. Some coordinators reported that their EHDI program too often loses contact with the family due to lack of documentation or because the hospital does not conduct their own rescreen as part of a standard protocol. In rural areas, EHDI coordinators report that these issues may be compounded because of a shortage of qualified practitioners (e.g., physicians, pediatric audiologists, early interventionists).

Home births $(12 \%)$ were cited as a weakness in some states, and many appear to lack an effective system for screening the hearing of infants born in the home. EHDI coordinators noted that home births were most likely to result in loss to follow-up. Other respondents noted that home births were increasing and that the majority of those infants were not screened for hearing loss.

Other areas cited as weaknesses included insufficient legislation/regulations $(9 \%)$, inadequate equipment $(8 \%)$, challenges related to screening $(8 \%)$, insufficient number of service providers (7\%), and weaknesses in EHDI operations (5\%). Reported legislative and regulatory concerns included the absence of state mandates for newborn hearing screening or audiologists not being required to report diagnosis. Equipment concerns included outdated equipment, abnormally high refer rates, and lack of instrumentation to provide auditory brainstem response (ABR) screening in the NICU. Abnormally high refer rates or excessive rescreenings to obtain a "pass" were primary concerns related to screening. EHDI coordinators also reported that there were not enough qualified pediatric audiologists and a high turnover of hospital staff. Not having adequate EHDI staff, materials, and personnel that are culturally and linguistically appropriate $(5 \%)$ and inadequate funding $(4 \%)$ were also areas identified as weaknesses.

\section{Opportunities for EHDI Programs}

The three opportunities most frequently cited were data collection $(25 \%)$, training and educational resources (19\%), and legislation (14\%) (Table 4). Twenty-five percent of respondents reported that improved data collection and tracking was a major opportunity for the EHDI program. Coordinators reported efforts to integrate existing screening databases (e.g., UNHS/EHDI, Newborn Metabolic Screening) with other vital records (e.g., electronic birth certificates, immunization registries) created opportunities for increased effectiveness and efficiency. State EHDI coordinators also expressed an ongoing need to update existing procedures by reducing "pen and paper" procedures in favor of web-based systems.

Training and educational resources were reported as opportunities by $19 \%$ of the respondents. State EHDI coordinators described a range of training 
Table 4. Top three themes for opportunities

\begin{tabular}{ll}
\hline \multicolumn{1}{c}{ Themes } & \multicolumn{1}{c}{ Descriptions } \\
\hline Data Collection & Automation of data collection process. \\
& Opportunity to upgrade the existing system. \\
Integration with the immunization registry. & \\
Training \& Educational Resources & $\begin{array}{l}\text { Training resources available through NCHAM. } \\
\text { Participating in NICHQ initiative. } \\
\text { Webinars in place to increase training. } \\
\text { Legislation }\end{array}$ \\
& Legislative expansion of the program. \\
& Good legislative support. \\
& Newborn hearing screening law took effect in 2008.
\end{tabular}

and educational resources that were available, including those provided by NCHAM and by the National Initiative for Children's Healthcare Quality (NICHQ). Other coordinators reported expanded use of webinars and training targeted to physicians, nursing staff, and volunteer screeners.

Legislation was the third most frequent opportunity, cited by $14 \%$ of respondents. Coordinators reported success in securing state legislation and mandates in support of UNHS programs, which created opportunities to update and strengthen existing policies. In states where UNHS legislation has not been implemented, EHDI coordinators reported opportunities for collaboration with other key stakeholders and organizations, such as the March of Dimes, to secure passage of legislation.

Other opportunities included issues related to follow-up (13\%), funding $(8 \%)$, relationships $(8 \%)$, and infrastructure $(6 \%)$. Related to follow-up, EHDI coordinators reported opportunities to work with midwives, health departments, diagnostic centers, smaller hospitals, and regional centers to help them with follow-up rescreens in the infant and toddler's community. Opportunities to apply for federal grants and assistance were also reported. Relationship opportunities included networks, engaged advisory boards, and meetings with community elders. Opportunities related to infrastructure included updated guidelines, staff with expertise in EHDI, and shared child health records.

\section{Threats to EHDI Programs}

The top most commonly cited threats were funding (42\%), personnel $(22 \%)$, and follow-up (9\%) (Table 5). Forty-two percent of the respondents reported that a lack of funding and other resources were a serious threat to UNHS programs. State budget cuts and funding shortages have led to program instability, staff shortages, reduced resources for screening equipment maintenance/ replacement, travel, and training/staff development. Most states expressed 
Table 5. Top three themes for threats

\begin{tabular}{ll}
\hline \multicolumn{1}{c}{ Themes } & \multicolumn{1}{c}{ Descriptions } \\
\hline Funding & $\begin{array}{l}\text { Lack of funding for new equipment. } \\
\text { Major budget shortfalls in the state. } \\
\text { Reduced and/or loss of state \& federal funding. } \\
\text { Personnel Issues }\end{array}$ \\
& $\begin{array}{l}\text { Changes in staff \& staff shortages at various hospitals. } \\
\text { Lack of administrative support. } \\
\text { Follow-Up Issues }\end{array}$ \\
& $\begin{array}{l}\text { State personnel layoffs. } \\
\text { More home births occurring in the state. }\end{array}$ \\
& Families that do not speak English. \\
& Communities that use unlicensed midwives. \\
\hline
\end{tabular}

concerns about long-term program viability if continued funding cuts occur at both the federal and state levels.

Personnel issues were cited by $22 \%$ of the states EHDI coordinators as a significant threat. Coordinators expressed concern about the shortage of pediatric audiologists, and the high rate of staff turn-over in hospital-based UNHS programs and at the state-level within EHDI programs (e.g., state EHDI coordinator, data entry specialists, technical assistance, administrative support). Several respondents noted that staff shortages and turnover have weakened their state wide programs.

Follow-up issues were reported as a threat by $9 \%$ of the respondents. EHDI coordinators reported that infants are often born in metropolitan hospitals or birthing centers, but reside in rural areas within the state that have limited access to follow-up services. Other respondents acknowledged difficulty providing screening services for infants born in the home. Non-English speaking families were cited as a challenge by some respondents, as infants born to these families often posed challenges related to follow-up. Finally, some EHDI coordinators reported difficulty obtaining screening data from religious or cultural groups due to the use of unlicensed midwives.

Also cited as threats were issues related to data collection $(8 \%)$, legislation (5\%), and equipment (5\%). Related to data collection, EHDI coordinators noted problems with computer firewalls and e-mail systems, data transfer from one system to another, and lack of automated data management system. Other threats included the lack of mandates to report data, confidentiality laws that make it difficult to share records, lack of standards for equipment calibration, and the need for hearing screening technology to identify mild hearing loss.

\section{TOWS Analysis Matrix}

By applying the TOWS analysis matrix to the themes identified in the SWOT analysis, specific recommendations, or "strategic options," were derived from 
the internal and external environmental factors identified by the EHDI coordinators. By systematically pairing the four categories of the SWOT analysis, recommendations can be derived to maximize resources and effect positive change. Based on the SWOT themes, 12 individual recommendations emerged (Table 6). To maximize opportunities, building strengths can maximize efficiency and lead to positive change (S-O strategy). For example, by requiring systematic data collection and reporting, existing screening practices will have a tool with which to make sound decisions on areas that focus on quality improvement. To overcome weaknesses, opportunities must be pursued (W-O strategy). For instance, by reaching out to key stakeholders and training programs, the state can provide quality training and educational resources related to UNHS to providers and families in the state. Identifying ways to use strengths can reduce vulnerability to threats (S-T strategy). For example, by engaging stakeholders through ongoing collaboration, there should be an improvement with data collection and tracking, which will reduce loss to follow-up. Finally, establishing defensive plans will help to prevent weaknesses from making programs and services susceptible to threats (W-T strategy). For example, developing cooperative agreements with other state agencies could improve integration of data collection processes with existing databases, or changes to existing legislation could help with program sustainability and funding. From the 12 recommendations, 3 strategies emerged to enhance UNHS initiatives. Specifically, states should: 1) require systematic data collection, reporting, and automation as well as integration of UNHS database systems with other public health initiatives; 2 ) leverage existing state legislative support to update and expand current UNHS polices and to garner increased resources (e.g., personnel, funding); and 3) reduce loss to follow-up by integrating and centralizing data collection and tracking among various agencies, and by addressing the screening needs of special populations (e.g., home births, non-English speaking families, and religious and culturally diverse groups).

\section{Limitations}

Limitations of this study include those inherent in the application of a SWOT/TOWS analysis when applied to rapidly changing conditions. SWOT analysis is a subjective tool that explores conditions that are perceived rather than objectively measured. In this sense, the SWOT analysis is influenced by the perspectives of those who completed the surveys as well as those who analyzed the responses. Furthermore, SWOT analysis characterizes the situation at a specific point in time. In addition, SWOT analysis, as applied here, resulted in overlapping themes within a category. For example, funding, legislation, and follow-up could each be identified as a strength, weakness, opportunity, or threat (see White \& Blaiser, 2011, for additional information regarding the selection of SWOT/TOWS for this study and further comment regarding interpretation and application of findings). 
Table 6. TOWS analysis matrix for newborn hearing screening

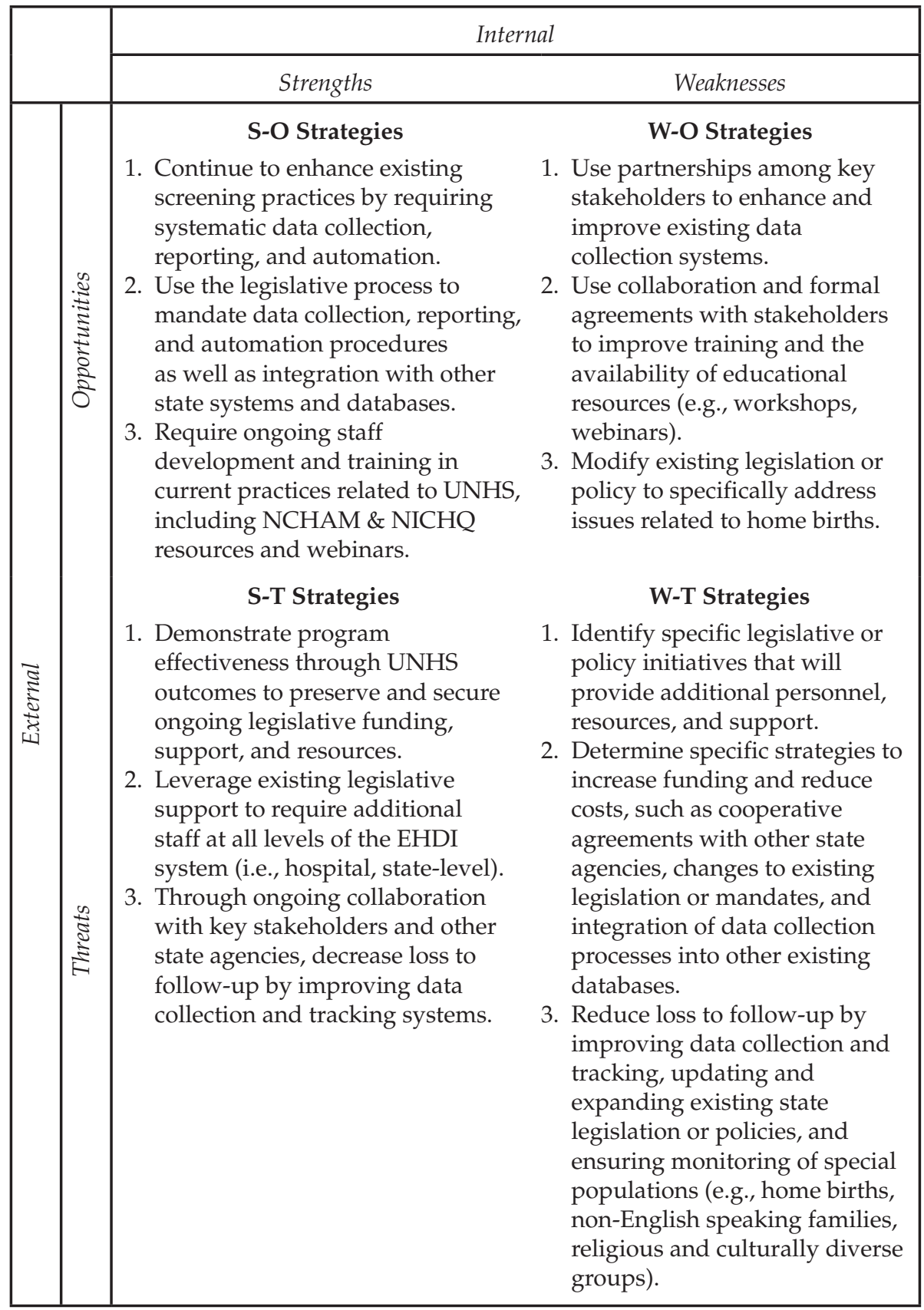

$\mathrm{S}=$ Strength $\mathrm{W}=$ Weakness $\mathrm{O}=$ Opportunity $\mathrm{T}=$ Threat 


\section{Summary}

For this analysis, 49 state EHDI coordinators provided 293 responses in evaluating newborn hearing screening practices. These data suggest that the implementation of newborn hearing screening programs in hospitals and birthing centers have been overwhelmingly successful. That is, infants are being screened at relatively high rates in most states, at times reaching $99 \%$. Although high rates of newborn hearing screening are now common, challenges exist in ensuring that infants who do not pass a hearing screen receive timely audiological assessment and, when necessary, appropriate intervention services. Although some states have benefited from strong data collection and tracking of newborns who do not pass their hearing screen, many struggle with decentralized, antiquated, and ineffective systems. Furthermore, the lack of well-qualified pediatric audiologists and early intervention providers (e.g., speech-language pathologists, teachers of the deaf) complicate successful follow-up. Special populations, such as infants born in the home, non-English speaking families, and the unique characteristics associated with certain religious or culturally diverse groups, also pose challenges to early identification and follow-up.

Budget cuts at both state and federal levels are requiring EHDI programs to do more with less. To ensure the long-term viability of UNHS programs and, by extension, state EHDI systems, coordinators must seek partnerships with key stakeholders, such as hospitals/birthing centers, state agencies, universities, and direct service providers. At the same time, EHDI coordinators must leverage the many successes that have been achieved through earlier identification of hearing loss to secure increased funding and resources from all possible local, state, and federal sources.

\section{Acknowledgement}

The work reported in this article was funded in part by the Maternal and Child Health Bureau under Cooperative Agreement \#U52MC04391 with the National Center for Hearing Assessment and Management at Utah State University. The opinions expressed in the article are those of the authors and do not necessarily reflect those of the Bureau.

\section{References}

Calderon, R. (2000). Parental involvement in deaf children's education programs as a predictor of child's language, early reading, and social-emotional development. Journal of Deaf Studies and Deaf Education, 5(2), 140-155.

Calderon, R., \& Naidu, S. (2000). Further support for the benefits of early identification and intervention for children with hearing loss. The Volta Review, 100(5), 3-84. 
Centers for Disease Control and Prevention (CDC). (2008). Early hearing detection and intervention hearing screening and follow-up survey. Retrieved May 19, 2011, from http:/ / www.cdc.gov/ncbddd/ehdi/data.htm.

Centers for Disease Control and Prevention (CDC). (2010a). National early hearing detection and intervention (EHDI) goals. Retrieved May 19, 2011, from http://www.cdc.gov/ncbddd/hearingloss/ehdi-goals.html.

Centers for Disease Control and Prevention (CDC). (2010b). Annual data: Early hearing detection and intervention (EHDI) program. Retrieved May 24, 2011, from http://www.cdc.gov/ncbddd/hearingloss/ehdi-data.html.

Joint Committee on Infant Hearing (JCIH). (2007). Year 2007 position statement: Principles and guidelines for early hearing detection and intervention programs. Pediatrics, 120(4), 898-921.

Moeller, M. P. (2000). Early intervention and language development in children who are deaf and hard of hearing. Pediatrics, 106, 1-9.

National Center for Hearing Assessment and Management (NCHAM). (2010). State EHDI/UNHS mandates: Summary table. Retrieved May 17, 2011, from http://www.infanthearing.org/legislative/summary/index.html.

White, K. R. (2004). Early hearing detection and intervention programs: Opportunities for genetic services. American Journal of Medical Genetics, 130A, 29-36.

White, K., \& Blaiser, K. (2011). Strategic planning to improve early hearing detection and intervention programs. The Volta Review, 111(2), 83-108.

White, K. R., Forsman, I., Eichwald, J., \& Muñoz, K. (2010). The evolution of early hearing detection and intervention programs in the United States. Seminars in Perinatology, 31(2), 170-189.

Yoshinaga-Itano, C. Sedey, A. L., Coulter, B. A., \& Mehl, A. L. (1998). Language of early- and later-identified children with hearing loss. Pediatrics, 102, $1168-1171$. 


\title{
A Systematic Analysis of Audiological Services in EHDI
}

\author{
Karen F. Muñoz, Ed.D., CCC-A; \\ Tamala S. Bradham, Ph.D., CCC-A; and Lauri Nelson, Ph.D.
}

State coordinators of early hearing detection and intervention (EHDI) programs completed a strengths, weaknesses, opportunities, and threats, or SWOT, analysis that examined 12 areas within state EHDI programs. For audiological evaluation and services, 299 items were listed by 49 coordinators, and themes were identified within each SWOT category. A threats, opportunities, weaknesses, and strengths, or TOWS, analysis yielded 12 recommendations that focused on similar themes. Out of these 12 recommendations, 3 distinct strategies emerged: 1) states need to address data management needs; 2) professional educational opportunities in pediatric audiology should be enhanced; and 3) funding opportunities for audiological services should be expanded. Addressed appropriately, these recommendations can substantially strengthen audiological services needed by state EHDI programs.

\section{Introduction}

The advent of newborn hearing screening (NHS) has changed the face of pediatric audiological practices. Prior to NHS, the average age of hearing loss diagnosis was 2 to 3 years of age; however, this has rapidly decreased to 2 to 3 months of age due to policy initiatives, federal support for early hearing detection and intervention (EHDI) programs, and state legislation (Sininger, Martinez, Eisenberg, Christensen, Grimes, \& Hu, 2009; White, Forsman, Eichwald, \& Muñoz, 2010). The first NHS state mandates were passed in the 1990s, with the majority of the mandates being implemented between 1999 and 2005. Currently, there are 43 states and territories that mandate NHS (National Center for Hearing Assessment and Management [NCHAM], 2010).

Karen F. Muñoz, Ed.D., CCC-A, is an Assistant Professor in the Department of Communicative Disorders and Deaf Education at Utah State University. Tamala S. Bradham, Ph.D., CCC-A, is an Assistant Professor in the School of Medicine, Department of Hearing and Speech Sciences, at Vanderbilt University. Lauri Nelson, Ph.D., is an Assistant Professor in the Department of Communicative Disorders and Deaf Education at Utah State University. Correspondence concerning this manuscript should be directed to Dr. Muñoz at karen.munoz@usu.edu. 
This recent and rapid change in the age of identification has resulted in a widespread need for audiologists skilled in providing services for the infant population.

Optimal speech, language, and academic outcomes for children with hearing loss are attainable goals. Yet a 2007 EHDI hearing screening and follow-up survey revealed that for $46 \%$ of babies who did not pass the NHS, follow-up information was not reported to the state EHDI program rendering it difficult to determine how many babies did not receive follow-up testing (Centers for Disease Control and Prevention [CDC], 2010; Mason, Gaffney, Green, \& Grosse, 2008). In addition, for those babies reported to have received diagnostic testing, 34\% were not tested by 3 months of age, the goal set forth in the Joint Committee on Infant Hearing (JCIH) 2007 guidelines (JCIH, 2007). The provision of timely and comprehensive diagnostic testing affects the window of opportunity for language development following a failed screening, and facilitates the parent's ability to capitalize on the time provided by early identification (American Speech-Language-Hearing Association [ASHA], 2008). State EHDI coordinators face various challenges in determining how to effectively connect parents to necessary services throughout the EHDI system.

Audiological services are an integral part of the EHDI process, from screening through intervention. The services provided by an audiologist at each point in time may enhance or limit the impact of intervention. The audiologist's role in NHS may include oversight of hospital screening programs, protocol development, training of screeners, and the screening process itself (American Academy of Audiology [AAA], 2010). When a baby does not pass NHS, referral to an audiologist qualified to provide pediatric assessment and equipped with the appropriate facilities needs to occur prior to 3 months of age (ASHA, 2004; JCIH, 2007).

Once a hearing loss is identified, the audiologist is responsible for medical referrals to determine the etiology of the hearing loss and any co-existing conditions that need to be considered during follow-up care. It is essential that the audiologist communicate test results to the primary care physician and maintain dialogue with other service providers. In addition, according to JCIH (2007), infants and toddlers with permanent hearing loss should be referred to an otolaryngologist with knowledge of pediatric hearing who can perform medical assessments and make referrals for additional evaluations, such as to a geneticist and an ophthalmologist, as well as provide medical clearance for amplification if parents choose to pursue use of amplification. Unfortunately there are gaps in physician attitudes, practices, and knowledge related to EHDI, as identified by focus group findings (Moeller, Eiten, White, \& Shisler, 2006) and through a national survey of physicians (Moeller, White, \& Shisler, 2006). Because audiologists are the professionals who diagnose the hearing loss and often initiate the referral process when a hearing loss is identified, they are well positioned to provide educational outreach to physicians 
and others in their state or locality, or on a case-by-case basis (Muñoz, Shisler, Moeller, \& White, 2009).

In addition to medical referrals, many state NHS mandates require audiologists to report the results of outpatient screening and assessment to their state EHDI program. State EHDI coordinators are responsible for tracking the outcomes of children with hearing loss and rely on timely and accurate reporting for outcomes that they are then responsible for reporting to the CDC. Outcome data inform the EHDI system and is the mechanism by which EHDI coordinators identify problems within the system and determine where to focus their resources to improve systems of care for children with hearing loss and their families.

When a hearing loss is identified, the audiologist is also responsible for making a referral to the Part $C$ early intervention system within two working days of the identification (National Early Childhood Technical Assistance Center, 1999). Following the diagnosis of a hearing loss, parents often have an overwhelming need for information, unbiased guidance, and emotional support. A prompt connection to early intervention providers offers a mechanism with which to address parents' needs and maximize the opportunity to access early intervention as a result of early diagnosis. Audiologists are also involved in the intervention process for children whose parents choose to pursue a listening and spoken language approach through the use of hearing technology. For those children, hearing aid fitting should occur within 1 month of confirmation of the hearing loss $(\mathrm{JCIH}, 2007)$. Communicating with EHDI coordinators about entry into early intervention programs and reporting when children are fitted with hearing aids will provide outcome data to determine if EHDI goals are being met. Audiological services are integral to the success of the EHDI process; therefore, these services need to be evaluated to determine if there are areas that need improvement.

The purpose of this study was to complete a systematic review using a strengths, weaknesses, opportunities, and threats, or SWOT, and a threats, opportunities, weaknesses, and strengths, or TOWS, analysis to assess the current EHDI practices related to audiological services, and to generate recommendations to improve effectiveness and outcomes for infants referred from newborn hearing screening.

\section{Methods}

Fifty-one EHDI coordinators were asked to complete an online survey. To examine issues related to audiological evaluation and services, the survey asked for EHDI coordinators to report at least 1 strength, weakness, opportunity, and threat for use in strategic planning. The responses obtained were reviewed by a panel of experts and categorized into common themes within four strategic planning areas. To generate recommendations from the SWOT analysis, a TOWS matrix was used to match identified strengths with 
opportunities (S-O strategy), strengths with threats (S-T strategy), weaknesses with opportunities (W-O strategy), and weaknesses with threats (W-T strategy). For an in-depth review of the SWOT/TOWS analysis and its use in the current study, the reader is referred to White and Blaiser, 2011.

\section{Results}

Forty-nine state EHDI coordinators (96\%) completed the audiological evaluation and services component of the SWOT questionnaire. Respondents identified 76 items in the strength category, 92 items in the weaknesses category, 64 items in the opportunities category, and 67 items in the threats category for a total of 299 responses. Based on the responses, the following number of themes was identified in each category: 7 for strengths, 5 for weaknesses, 6 for opportunities, and 8 for threats (Table 1). Each category also had a miscellaneous section that included no comment, N/A, or items that did not fit within the themes identified.

Table I. Frequency of themes within each SWOT category $(n=299)$

\begin{tabular}{clc}
\hline SWOT category & \multicolumn{1}{c}{ Themes } & Frequency $n(\%)$ \\
\hline Strengths $(\mathrm{n}=76)$ & Data Reporting and Tracking & $16(21 \%)$ \\
& Availability of Services & $13(17 \%)$ \\
& Professional Communication/Collaboration & $13(17 \%)$ \\
& Miscellaneous / No Comments & $10(13 \%)$ \\
& Comprehensive Audiological Services & $11(14 \%)$ \\
& Skilled/Dedicated Audiologists & $9(12 \%)$ \\
& Funding and Reimbursement & $4(5 \%)$ \\
Weaknesses $(\mathrm{n}=92)$ & Availability of Services & $46(50 \%)$ \\
& Data Reporting and Tracking & $20(22 \%)$ \\
& Miscellaneous/No Comment & $11(12 \%)$ \\
& Funding and Reimbursement & $8(9 \%)$ \\
Opportunities $(\mathrm{n}=64)$ & Timeliness of Services & $7(8 \%)$ \\
& Data Reporting and Tracking & $19(30 \%)$ \\
& Education and Training & $15(23 \%)$ \\
& Funding and Reimbursement & $9(14 \%)$ \\
& Professional Communication/Collaboration & $8(13 \%)$ \\
& Miscellaneous/No Comment & $8(13 \%)$ \\
& Facilities and Equipment & $5(4 \%)$ \\
& Funding and Reimbursement & $17(25 \%)$ \\
Threats $(\mathrm{n}=67)$ & Non-Compliance to EHDI System & $14(21 \%)$ \\
& Availability of Services & $13(19 \%)$ \\
& Miscellaneous/No Comment & $10(15 \%)$ \\
& Equipment/Protocol Issues & $5(7 \%)$ \\
& Training & $3(4 \%)$ \\
& Patient-Related Challenges & $3(4 \%)$ \\
& Legislation & $2(3 \%)$ \\
\hline
\end{tabular}




\section{Strength Characteristics}

The three most frequently cited strengths were data reporting and tracking $(21 \%)$, availability of services $(17 \%)$, and professional communication and collaboration (17\%) (Table 2). Data reporting and tracking are essential for state EHDI coordinators for them to know the status of each infant who needs services. The key elements that contributed to making reporting and tracking a strength is mandated reporting, a dedicated process for monitoring and tracking evaluations, the ability to generate an online report, and the ability to provide audiologists with quarterly feedback on EHDI outcomes.

The elements reported as instrumental to making service ability a strength included more pediatric audiologists available than in the past, and having diagnostic centers throughout the state that included comprehensive audiological services and qualified audiologists. Respondents also mentioned that it is helpful when a list is available for parents and stakeholders (e.g., physicians) to identify the audiologists qualified to provide audiological services. Communication between the audiologists and the EHDI program, and having input from audiologists regarding protocols and policies, were also noted as key elements in achieving successful professional communication related to audiological services.

Other strengths included availability of comprehensive audiological services $(14 \%)$, skilled/dedicated audiologists $(12 \%)$, and availability of sufficient funding and reimbursement $(5 \%)$, such as having state funding pay for the hearing evaluation through the NHS program, Title V, or early intervention services.

\section{Weakness Characteristics}

The most frequently cited weaknesses were availability of services (50\%), data reporting and tracking (22\%), and funding and reimbursement $(9 \%)$ (Table 3). Half of the respondents noted that availability of services was a

Table 2. Top three themes for strengths

\begin{tabular}{ll}
\hline \multicolumn{1}{c}{ Themes } & \multicolumn{1}{c}{ Description } \\
\hline Data Reporting and Tracking & $\begin{array}{l}\text { Mandated reporting. } \\
\text { Dedicated tracking process. } \\
\text { Online reporting. }\end{array}$ \\
& $\begin{array}{l}\text { Access throughout state. } \\
\text { Availability of Services }\end{array}$ \\
& $\begin{array}{l}\text { Comprehensive testing/expertise. } \\
\text { Commitment to reporting outcomes. }\end{array}$ \\
Professional Communication/Collaboration & $\begin{array}{l}\text { Audiologist input on policies and } \\
\text { protocols. }\end{array}$ \\
\hline
\end{tabular}


Table 3. Top three themes for weaknesses

\begin{tabular}{ll}
\hline \multicolumn{1}{c}{ Themes } & \multicolumn{1}{c}{ Description } \\
\hline Availability of Services & Limited access in rural areas. \\
& Transportation limitations. \\
& Lack of skilled audiologists. \\
& Lack of appropriate equipment. \\
& Delays in appointment availability. \\
Data Reporting and Tracking & Lack of reporting mandate. \\
& Lack of web-based system. \\
Funding and Reimbursement & Lack of Medicaid providers. \\
& No or limited funding for uninsured.
\end{tabular}

weakness. The elements that limited availability of service as a weakness included limited access to diagnostic services in rural areas, lack of transportation to providers, and limited service locations. This includes a shortage of skilled pediatric audiologists with the appropriate equipment and facilities needed to provide comprehensive testing.

Elements that interfered with adequate data reporting and tracking included inconsistent reporting, the lack of a statewide reporting mandate, and the lack of an online system for reporting outcomes. Funding and reimbursement were reported by some states as a weakness; specific reasons why included a lack of providers that accept Medicaid, poor reimbursement rates for Medicaid, and no payment source for children who are uninsured.

Timeliness of services $(8 \%$ ) was also noted, particularly related to delays in availability of appointments for children on Medicaid.

\section{Opportunities for EHDI Programs}

The most frequently cited opportunities to improve audiological services were data reporting and tracking (30\%), education and training $(23 \%)$, and funding and reimbursement (14\%) (Table 4). The elements viewed as creating opportunities for data reporting and tracking were improvements in the database

Table 4. Top three themes for opportunities

\begin{tabular}{ll}
\hline \multicolumn{1}{c}{ Themes } & \multicolumn{1}{c}{ Description } \\
\hline Data Reporting and Tracking & Improve database/protocol. \\
& Web-based reporting. \\
& Legislation to mandate reporting. \\
Education and Training & Expand training delivery options. \\
Funding and Reimbursement & Funding for diagnostic evaluation. \\
& Funding for training. \\
& Address Medicaid reimbursement. \\
\hline
\end{tabular}


and reporting protocol, online reporting, and updating legislation to mandate reporting. Education and training were viewed as an avenue to improve EHDI systems through the use of various educational mechanisms (e.g., the state department of health, Au.D. programs, teleconferences, and audiology consultants). Funding and reimbursement was also mentioned by some states as an opportunity, specifically identifying funding for audiological evaluations and training as well as improving reimbursement rates for children on Medicaid.

Other opportunities included professional communication/collaboration $(13 \%)$, specifically through identifying all pediatric audiologists in the state, providing a directory or e-mail distribution list of pediatric audiologists to improve communication among providers, and contacting audiologists on a regular basis. Facilities and equipment were also mentioned (4\%), specifically upgrading equipment (e.g., more portable) and opening new diagnostic facilities.

\section{Threats to EHDI Programs}

The three most frequently cited threats were funding and reimbursement $(25 \%)$, non-compliance with the EHDI system $(21 \%)$, and availability of services (19\%) (Table 5). Funding and reimbursement issues included a loss of audiology providers (particularly those that accept Medicaid), a lack of insurance coverage, and state budget deficits. Concerns related to noncompliance with the EHDI system included audiologists not recognizing the need for continuing education, inappropriate assessment procedures, and unwillingness to report outcomes to the state EHDI coordinator. The elements that were viewed as threats to the availability of services included lack of access to audiological assessment, a shortage of qualified audiologists, use of inappropriate protocols, and a lack of appropriate instrumentation for pediatric audiology.

A few state EHDI coordinators expressed concern about equipment/protocol issues $(7 \%)$, specifically the use of otoacoustic emissions tests (OAE) to rescreen an infant who did not pass the first screening test. Also mentioned was training $(4 \%)$, such as not having an ongoing training plan or an

Table 5. Top three themes for threats

\begin{tabular}{ll}
\hline \multicolumn{1}{c}{ Themes } & \multicolumn{1}{c}{ Description } \\
\hline Reimbursement & Loss of Medicaid providers. \\
& Lack of insurance coverage. \\
& Budget deficits. \\
Non-Compliance with EHDI System & Unwillingness to report results. \\
& Lack of recognition of need for training. \\
Availability of Services & Lack of equipment. \\
& Use of inappropriate protocols. \\
& Lack of skilled audiologists. \\
\hline
\end{tabular}


audiology graduate training program in the state, patient-related challenges $(4 \%)$, such as transportation and language barriers, and legislation $(3 \%)$, specifically due to a lack of mandated reporting and regulation.

\section{TOWS Analysis Matrix}

By applying the TOWS analysis to the themes identified in the SWOT analysis, specific recommendations, or "strategic options," were derived from the internal and external environmental factors identified by the EHDI coordinators. By systematically pairing the four categories of the SWOT analysis, recommendations can be derived to maximize resources and effect positive change. Based on the SWOT themes, 12 individual recommendations were made (Table 6). To maximize opportunities, addressing strengths can maximize

Table 6. TOWS analysis matrix for audiological services

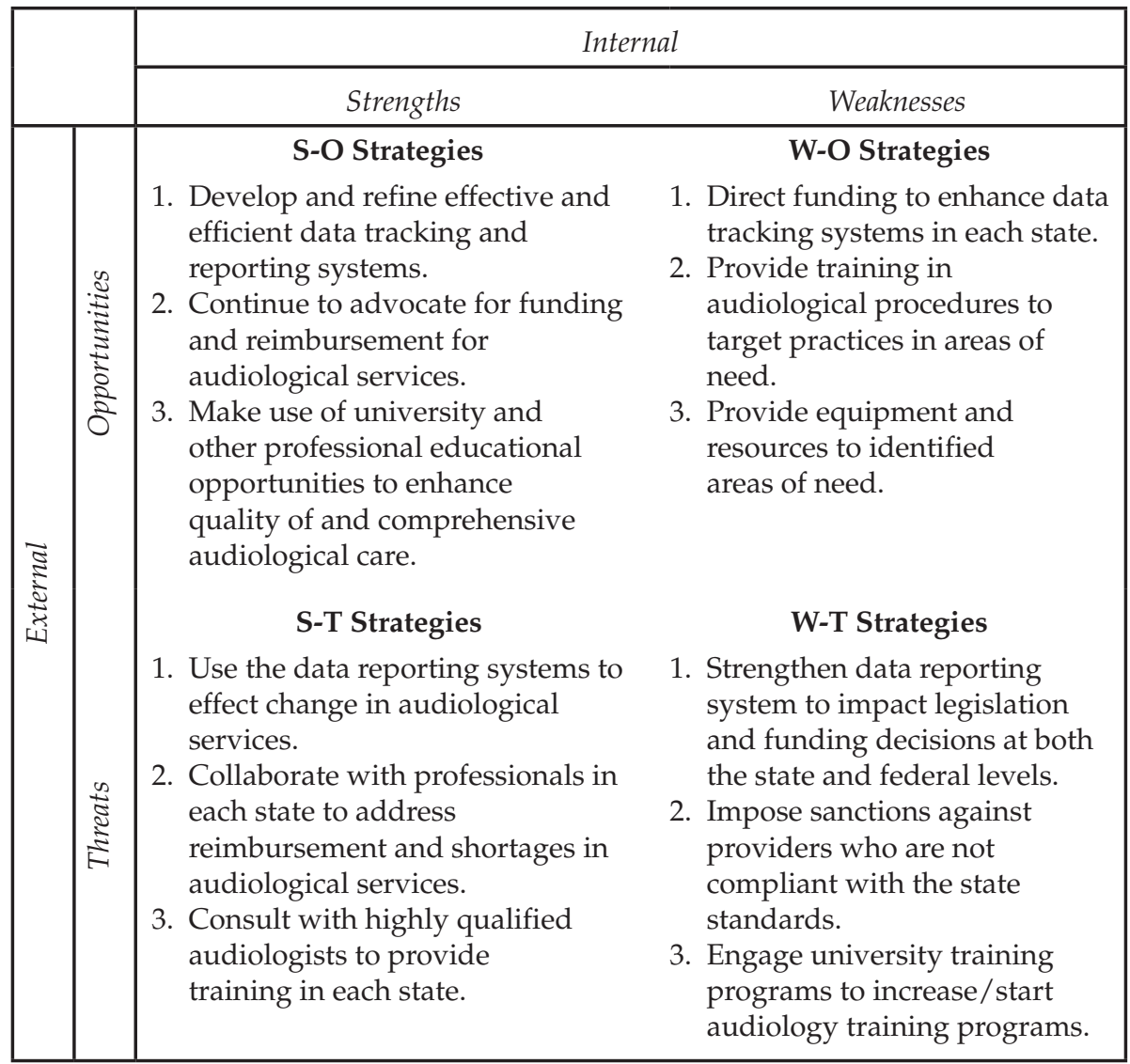

$\mathrm{S}=$ Strength $\mathrm{W}=$ Weakness $\mathrm{O}=$ Opportunity $\mathrm{T}=$ Threat 
efficiency and lead to positive change (S-O strategy). For example, to enhance quality and comprehensive audiological care, states should make use of university and other professional educational opportunities to enhance audiological care. To overcome weaknesses, it is necessary to pursue opportunities (W-O strategy). For example, by directing funding to a data management system, better reporting of audiological results can be provided to the EHDI coordinators, audiologists and medical homes in the state. Identification of ways to use strengths will ultimately reduce vulnerability to threats (S-T strategy). For example, to address pediatric shortages and reimbursement for services, audiologists should collaborate with other audiologists and professionals in the state to address these issues. Finally, establishing defensive plans will help prevent weaknesses from making policies and services susceptible to threats (W-T strategy). For example, strengthening the state data reporting system could positively impact legislation and funding decisions at both the state and federal levels. Out of these 12 recommendations, 3 distinct strategies for improving audiological evaluations and services emerged. Specifically, states need to: 1) address their data management needs related to audiological assessment and reporting; 2) enhance professional educational opportunities in pediatric audiology; and 3) improve funding for audiological services.

\section{Limitations}

Limitations of this study include those inherent in the application of SWOT/ TOWS analysis when applied to rapidly changing conditions. SWOT analysis is a subjective tool that explores conditions that are perceived rather than objectively measured. In this sense, the SWOT analysis is influenced by the perspectives of those who completed the surveys as well as those who analyzed the responses. Furthermore, SWOT analysis characterizes the situation at a specific point in time. In addition, SWOT analysis, as applied here, resulted in overlapping themes within a category. For example, funding could be identified in all four categories as a strength, weakness, opportunity, or threat (see White \& Blaiser, 2011, for additional information regarding the selection of SWOT/TOWS for this study and further comment regarding interpretation and application of findings).

\section{Summary}

As a result of NHS, audiologists who work with children are called upon to routinely provide audiological services for infants. The skills, expertise, and equipment required to assess the hearing of an infant differ significantly from those needed to provide behavioral hearing assessments of toddlers and older children. Some audiologists are encountering demand to provide infant services without the requisite knowledge, skills, and equipment to appropriately meet this need. A comprehensive hearing assessment provides the foundation 
from which amplification and intervention decisions are determined. Audiological assessment guidelines and specific test battery recommendations are available to audiologists (ASHA, 2004; JCIH, 2007). Additionally, there are specific procedures for fitting a child with hearing aids to ensure appropriate audibility (AAA, 2003; Scollie \& Seewald, 2001).

As revealed in the responses of state EHDI coordinators, incomplete testing or inappropriate test procedures can delay the diagnosis and intervention process. Furthermore, EHDI coordinators reported that it can be difficult to identify facilities that have appropriately qualified pediatric audiologists and the equipment needed to provide comprehensive testing. A study by Windmill \& Windmill (2006) provided a window into what this can mean for a state EHDI program; they surveyed facilities listed as providers of infant diagnostic testing in Kentucky to investigate if they were performing the diagnostic evaluation as recommended by the JCIH. Of the 42 facilities listed, 34 reported that they provided testing for infants. For those facilities that tested infants, when the complete test battery was considered only 3 facilities (8\%) were providing infant services that met the JCIH diagnostic test battery recommendations. A broader view of this issue was obtained through a national survey completed in 2009 investigating the diagnostic testing practices of pediatric audiology facilities in the United States. Of the 356 respondents, 304 reported that they provide diagnostic testing for infants. For those facilities that tested infants, results revealed that only $7 \%$ reported using all of the components of the JCIH recommended test battery (Muñoz \& Nelson, 2010). While the target recipients for both studies were pediatric audiology facilities, recognized as such by the state EHDI coordinators, 19\% of the facilities in Kentucky and $15 \%$ of the facilities in the national sample reported that they did not provide infant diagnostic services.

The inconsistency of comprehensive infant diagnostic test batteries being used is cause for alarm. Currently, there is not a standard mechanism in place to designate pediatric facilities specializing in infant audiological services. While procedures vary by state, EHDI coordinators rely primarily on audiologists to report that they are able to provide pediatric testing services. Audiologists, as with other professions, are bound by their professional code of ethics to self-monitor their professional services and maintain competence in their practice; however, EHDI coordinators have an oversight responsibility for the EHDI system in their state. When an EHDI coordinator identifies discrepancies, what is their role in monitoring quality? The EHDI system of care is evolving and because time is of the essence, there is an urgent need for the profession of audiology to assist in identifying a mechanism for parents and other stakeholders to locate those audiologists with the equipment and expertise to meet the needs of the pediatric population, and in particular the infant population following a failed NHS.

The responses provided by EHDI coordinators revealed that there is considerable variability among states with regard to audiological services and 
inconsistency within categories. For instance, data tracking and reporting was considered a strength when there was a dedicated tracking system and a reporting mandate in place in the state. Data tracking and reporting was considered a weakness when there was a lack of a reporting mandate in the state. At the same time it was viewed as an opportunity when there were plans to improve the data tracking and reporting process.

EHDI coordinators' statements in each of the SWOT areas provide insights into issues they view as critical to the success of state EHDI programs. An understanding of the specific aspects that make an issue a strength for some states and a weakness for others can serve as a guide for action planning to improve EHDI systems. Review of these issues among states provide a perspective that can help EHDI coordinators, policymakers, professionals, parents, and other stakeholders know where to focus their attention and efforts to improve audiology services in their state.

\section{Acknowledgement}

The work reported in this article was funded in part by the Maternal and Child Health Bureau under Cooperative Agreement \#U52MC04391 with the National Center for Hearing Assessment and Management at Utah State University. The opinions expressed in the article are those of the authors and do not necessarily reflect those of the Bureau.

\section{References}

American Academy of Audiology (AAA). (2003). Pediatric amplification protocol. Retrieved April 21, 2011, from http://www.audiology.org/searchcen ter/pages/results.aspx?k=pediatric\%20amplification\%20protocol.

American Academy of Audiology (AAA). (2010). Role of the audiologist in the newborn hearing screening program. Retrieved May 17, 2011, from http://www. audiology.org/resources/documentlibrary/Pages/NewbornHearing Screening.aspx.

American Speech-Language-Hearing Association (ASHA). (2004). Guidelines for the audiological management of children from birth to 5 years of age. Retrieved May 17, 2011, from http:/ / www.asha.org/docs/html/GL2004-00002.html.

American Speech-Language-Hearing Association (ASHA). (2008). Loss to followup in early hearing detection and intervention [technical report]. Retrieved June 6, 2011, from http:/ / www.asha.org/docs/html/tr2008-00302.html.

Centers for Disease Control and Prevention (CDC). (2010). Annual data. Early hearing detection and intervention (EHDI) program. Retrieved May 17, 2011, from http://www.cdc.gov/ncbddd/hearingloss/ehdi-data.html.

Joint Committee on Infant Hearing (JCIH). (2007). Year 2007 position statement: Principles and guidelines for early hearing detection and intervention programs. Pediatrics, 120(4), 898-921. 
Mason, C. A., Gaffney, M., Green, D. R., \& Grosse, S. D. (2008). Measures of follow-up in early hearing detection and intervention programs: A need for standardization. American Journal of Audiology, 17, 60-67.

Moeller, M. P., Eiten, L., White, K., \& Shisler, L. (2006). Strategies for educating physicians about newborn hearing screening. Journal of the Academy of Rehabilitative Audiology, 1(39), 11-32.

Moeller, M. P., White, K., \& Shisler, L. (2006). Primary care physicians' knowledge, attitudes, and practices related to newborn hearing screening. Pediatrics, 118, 1357-1370.

Muñoz, K., \& Nelson, L. (2010, March). Success and challenges to providing timely infant diagnostic hearing assessments. Paper presented at the meeting of Early Hearing Detection and Intervention, Chicago, IL.

Muñoz, K., Shisler, L., Moeller, M. P., \& White, K. (2009). Improving the quality of early hearing detection and intervention services through physician outreach. Seminars in Hearing, 30(3), 184-192.

National Center for Hearing Assessment and Management (NCHAM). (2010). State EHDI/UNHS mandates: Summary table. Retrieved May 17, 2011, from http://www.infanthearing.org/legislative/summary/index.html.

National Early Childhood Technical Assistance Center. (1999). Federal regulations for 34 CFR part 303, early intervention program for infants and toddlers with disabilities. Retrieved May 17, 2011, from http://www.nectac.org/ idea/303regs.asp.

Scollie, S. D., \& Seewald, R. C. (2001). Hearing aid fitting and verification procedures for children. In J. Katz (Ed.), The Handbook of Clinical Audiology, $5^{\text {th }} E d$ (pp. 687-706). Baltimore, MD: Lippincott, Williams, \& Wilkins.

Sininger, Y., Martinez, A., Eisenberg, L., Christensen, E., Grimes, A., \& Hu, J. (2009). Newborn hearing screening speeds diagnosis and access to intervention by 20-25 months. Journal of the American Academy of Audiology, 20(1), 49-57.

White, K., \& Blaiser, K. (2011). Strategic planning to improve early hearing detection and intervention programs. The Volta Review, 111(2), 83-108.

White, K., Forsman, I., Eichwald, J., \& Muñoz, K. (2010). The evolution of early hearing detection and intervention programs in the United States. Seminars in Perinatology, 31(2), 170-189.

Windmill, S., \& Windmill, I. (2006). The status of diagnostic testing following referral from universal newborn hearing screening. Journal of the American Academy of Audiology, 17(5), 367-378. 
The Volta Review, Volume 111(2), Summer 2011, 133-149

\title{
The EHDI and Early Intervention Connection
}

\author{
Lauri Nelson, Ph.D.; Tamala S. Bradham, Ph.D., CCC-A; and \\ K. Todd Houston, Ph.D., CCC-SLP, LSLS Cert. AVT
}

State coordinators of early hearing detection and intervention (EHDI) programs completed a strengths, weaknesses, opportunities, and threats, or SWOT, analysis that examined 12 areas within state EHDI programs. For the early intervention focus question, 48 coordinators listed 273 items, and themes were identified within each SWOT category. A threats, opportunities, weaknesses, and strengths, or TOWS, analysis yielded 8 recommendations that focused on similar themes. Out of these 8 recommendations, 3 distinct strategies emerged. Specifically, states should develop 1) an appropriate and effective eligibility and referral process to early intervention services; 2) a state advisory board or coalition group to identify funding needs and barriers, and to make recommendations; and 3) effective data management systems with reporting of data to key stakeholders. Addressed appropriately, these recommendations can strengthen access and entry into early intervention services through state EHDI programs.

\section{Introduction}

According to the report and recommendations of the 2004 NationalConsensus Conference on Effective Educational and Health Care Interventions for Infants and Young Children with Hearing Loss (Marge \& Marge, 2005), hearing health care and early intervention services for children with hearing loss should be accessible, family-centered, comprehensive, continuous, coordinated, compassionate, and culturally sensitive. The early hearing detection and intervention (EHDI) program encompasses a broad continuum of services from multiple agencies and service providers with the goal of ensuring that all infants and toddlers with hearing loss are identified as early as possible and provided

Lauri Nelson, Ph.D., is an Assistant Professor in the Department of Communicative Disorders and Deaf Education at Utah State University. Tamala S. Bradham, Ph.D., CCC-A, is an Assistant Professor in the School of Medicine, Department of Hearing and Speech Sciences at Vanderbilt University. K. Todd Houston, Ph.D., CCC-SLP, LSLS Cert. AVT, was an Assistant Professor in the Department of Communicative Disorders and Deaf Education at Utah State University at the time of this study and is currently at The University of Akron. Correspondence concerning this manuscript may be directed to Dr. Nelson at lauri.nelson@usu.edu. 
with timely and appropriate medical, audiological, and educational intervention (National Center for Hearing Assessment and Management [NCHAM], 2010). Stakeholders in EHDI programs include parents and families as well as a range of professionals, such as EHDI coordinators, early interventionists, physicians, audiologists, state department personnel, and education personnel. There are multiple components to EHDI programs, such as the initial newborn hearing screening, systems for follow-up for infants referred from newborn screening, medical evaluation, referral to medical specialists as appropriate, diagnostic audiological testing, and ongoing audiological management. The benefits of early medical and audiological management are maximized when accompanied by family-centered support throughout the diagnostic process, and family-centered early intervention services that use developmentally appropriate practices in the family's chosen mode of communication.

Early intervention is an integral component of EHDI programs and must include the provision of services that address the linguistic, communication, social, and cognitive needs of children who are deaf or hard of hearing (Hands \& Voices, 2010; Yoshinaga-Itano, 2003). Broadly defined, early intervention services are specialized health, educational, and therapeutic services designed to meet the needs of infants and toddlers, ages birth to 36 months, and their families. Early intervention is concerned with the basic skills of typical development during the first three years of life, such as 1) physical growth (e.g., reaching, rolling, crawling, walking); 2) cognitive development skills (e.g., thinking, learning, problem solving); 3) communication skills (e.g., talking, listening, understanding); 4) social/emotional development (e.g., learning to play, developing feelings of security); and 5) self-help (e.g., eating, dressing) (National Dissemination Center for Children with Disabilities, 2010).

Early intervention in the EHDI continuum of services begins with medical evaluation and completion of newborn hearing screening. Hospital-based infant hearing screening programs have been highly successful in the early identification of hearing loss in infants (White, 2004). Currently, every state in the United States has implemented either a mandatory or voluntary infant hearing screening program, partly due to the documented benefit in speech and language acquisition when the loss is identified and intervention begins within the first few months of life (NCHAM, 2010). In 1995, the percentage of newborns screened for hearing loss was just 3\%; over a decade later that number increased to 97\% (Centers for Disease Control and Prevention [CDC], 2010a). In those states where newborn hearing screening programs have been effectively implemented, the age of hearing loss identification has been reduced from an average of about 30 months of age to an average of 2-3 months of age (White, Forsman, Eichwald, \& Muñoz 2010). Although early identification is the critical first step, the full benefits of early diagnosis are realized only when effective and prompt early intervention services for language and cognitive development are implemented. Research shows that through early identification and family-centered early intervention, 
children with hearing loss experience better language, speech, cognitive, and social-emotional development than later-identified children (Kennedy, et. al., 2006; Moeller, 2000; Yoshinaga-Itano \& Gravel, 2001; Yoshinaga-Itano, Sedey, Coulter, \& Mehl, 1998).

EHDI goals for early identification and intervention for children with hearing loss should be consistent with the 2007 recommendations of the Joint Committee on Infant Hearing (JCIH), which was based on evidence showing that the optimal age for hearing loss identification and initiation of early intervention services was prior to 6 months of age (JCIH, 2007). JCIH reiterated the 1-3-6 model in which infants should be screened for hearing loss by 1 month of age, receive an appropriate audiological evaluation by 3 months of age, and, if hearing loss is confirmed, begin early intervention services by 6 months of age to "maximize linguistic competency and literacy development for children who are deaf or hard of hearing" (JCIH, 2007). Specifically, JCIH states that:

- All families of infants with any degree of bilateral or unilateral permanent hearing loss should be considered eligible for early intervention services.

- There should be recognized central referral points of entry to ensure specialty services for infants with confirmed hearing loss.

- Early intervention services for infants with confirmed hearing loss should be provided by professionals who have expertise in hearing loss, including educators of the deaf, speech-language pathologists, and audiologists.

- In response to a previous emphasis on "natural environment," the JCIH recommends that both home-based and center-based intervention options be offered.

Although recommendations regarding the importance of early intervention services are not new, evidence continues to suggest that not all children and families are receiving appropriate and timely early intervention services (CDC, 2010b; White, 2007). In 1990, the U.S. Department of Health and Human Services (DHHS) stated: "It is difficult if not impossible for hearing-impaired children to acquire the fundamental language, social and cognitive skills that provide the foundation for later schooling and success in society. When early identification and intervention occur, hearing-impaired children make dramatic progress, are more successful in school, and become more productive members of society" (p. 460). In a 2006 letter sent to all state early intervention programs, the assistant secretary of the U.S. Department of Education described a "growing national crisis in the provision of essential early intervention and health care services for infants and toddlers with hearing loss" and further noted that "although efforts to identify and evaluate hearing loss in young children have improved, there is still anecdotal evidence to suggest that many young children with hearing loss may not be receiving the early intervention or other services they need in a timely manner that 
will enable them to enter preschool and school ready to succeed" (Hager \& Giannini, 2006).

The program for infants and toddlers with disabilities (Part $\mathrm{C}$ of the Individuals with Disabilities Education Act [IDEA]) is a federal grant program that assists states in operating a comprehensive statewide program of early intervention services for infants and toddlers with disabilities, ages birth to 36 months, and their families. In order for a state to participate in the program it must assure that early intervention will be available to every eligible child and family. The 2007 national newborn hearing screening report from the CDC reported that $83 \%$ percent of infants diagnosed with hearing loss were referred to Part $C$ early intervention programs (CDC, 2008). However, only $61 \%$ of children identified with hearing loss actually had documented enrollment in a Part $\mathrm{C}$ early intervention program before 6 months of age (CDC, 2008). Thus, nearly $40 \%$ of children with hearing loss may not be receiving the services available to them, jeopardizing the benefits of early identification. As described in Section 631 of IDEA, Part C is designed to:

- Enhance the development of infants and toddlers with disabilities.

- Reduce the education costs to society by minimizing the need for special education services when they reach school age.

- Increase the potential for independent living in society.

- Facilitate the ability of families to maximize their child's development in the child's natural environment.

- Meet the needs of underrepresented populations.

Additional components are applied and relevant based on the child's disability.

Data indicating that nearly $40 \%$ of children with hearing loss are not enrolled in an early intervention program by 6 months of age (CDC, 2008) warrants an evaluation of potential barriers to accessing early intervention services. As described by White (2007), there are several components of a strong statewide EHDI system, including 1) specific well-defined eligibility criteria for children with hearing loss to participate in Part $C$ early intervention programs; 2 ) implementation of a comprehensive child-find and referral system with written policies and procedures for implementation; 3 ) a comprehensive plan for personnel training and development; 4) early intervention services that include optimal use of hearing technology; and 5) public awareness to inform stakeholders of current technology, policies, and recommendations. An evaluation of each component can help EHDI stakeholders identify areas of program improvement to ensure implementation of effective and appropriate early intervention services to all children with hearing loss.

The purpose of this study was to provide a systematic review using a strengths, weaknesses, opportunities, and threats, or SWOT, and a threats, opportunities, weaknesses, and strengths, or TOWS, analysis to determine the current EHDI practices in early intervention services, and to identify 
recommendations to improve program effectiveness that leads to better outcomes for infants and toddlers who are deaf and hard of hearing.

\section{Methods}

Fifty-one EHDI coordinators were asked to complete an online survey. To examine issues related to early intervention services, the survey asked for the EHDI coordinator to report at least 1 strength, weakness, opportunity, and threat for use in strategic planning. The responses obtained were reviewed by a panel of experts and categorized into common themes within four strategic planning areas. To generate recommendations from the SWOT analysis, a TOWS matrix was used to match identified strengths with opportunities (S-O strategy), strengths with threats (S-T strategy), weaknesses with opportunities (W-O strategy), and weaknesses with threats (W-T strategy). For an indepth review of the SWOT/TOWS analysis and its use in the current study, the reader is referred to White and Blaiser, 2011.

\section{Results}

Forty-eight EHDI coordinators (94\%) completed the early intervention section of the SWOT questionnaire. The respondents generated 81 items in the strengths category, 70 items in the weaknesses category, 58 items in the opportunities category, and 64 items in the threats category for a total of 273 response items. Based on the responses, several specific themes were generated for each category: 10 for strengths, 7 for weaknesses, 7 for opportunities, and 6 for threats (Table 1). Each category also had a miscellaneous section that included no comment, N/A, or items that did not fit within the themes identified.

\section{Strength Characteristics}

The three most frequently cited strengths were having an effective eligibility and referral process $(25 \%)$, collaboration between early intervention and EHDI programs $(20 \%)$, and having an adequate infrastructure for services $(15 \%)$ (Table 2). Twenty-five percent of EHDI coordinators indicated that timely and appropriate referral for early intervention for children with hearing loss is dependent on agencies having an effective eligibility and referral process. Once the diagnosis is made, all children with hearing loss should be eligible to access early intervention services. In 2004, when former President Bush signed legislation for the reauthorization of IDEA, it required states to specify the minimum components of their early intervention system. This allowed states to develop their own operational plans for serving children with disabilities, including establishing their own child eligibility criteria. As a result, definitions of eligibility differ significantly from state to state, including whether or not to serve infants who are considered at risk for a developmental 
Table I. Frequency of themes within each SWOT category $(n=273)$

\begin{tabular}{|c|c|c|}
\hline SWOT Category & Themes & Frequency $n(\%)$ \\
\hline \multirow[t]{10}{*}{ Strengths $(\mathrm{n}=81)$} & Effective Eligibility and Referral Process & $20(25 \%)$ \\
\hline & $\begin{array}{l}\text { Collaboration Between Early Intervention and } \\
\text { EHDI }\end{array}$ & $16(20 \%)$ \\
\hline & Adequate Infrastructure for Services & $12(15 \%)$ \\
\hline & Strong Program Services & $10(12 \%)$ \\
\hline & Highly Trained Service Providers & $5(6 \%)$ \\
\hline & Quality Improvement Projects & $5(6 \%)$ \\
\hline & Electronic Link for Data Sharing & $4(5 \%)$ \\
\hline & Family Support Programs & $3(4 \%)$ \\
\hline & Funding & $3(4 \%)$ \\
\hline & Miscellaneous/No Comment & $3(4 \%)$ \\
\hline \multirow[t]{7}{*}{ Weaknesses $(n=70)$} & $\begin{array}{l}\text { Poor Communication/Coordination of } \\
\text { Services }\end{array}$ & $16(23 \%)$ \\
\hline & No Electronic Link for Data Sharing & $13(19 \%)$ \\
\hline & Lack of Appropriately Trained Personnel & $12(17 \%)$ \\
\hline & Insufficient Access to Services & $12(17 \%)$ \\
\hline & Poor Relationships Among Stakeholders & $10(14 \%)$ \\
\hline & Funding & $4(6 \%)$ \\
\hline & Miscellaneous/No Comments & $3(4 \%)$ \\
\hline \multirow[t]{7}{*}{ Opportunities $(n=58)$} & Improved Relationships/Service Coordination & $21(36 \%)$ \\
\hline & Improve Systems for Electronic Data Sharing & $13(22 \%)$ \\
\hline & Improve Infrastructure and Procedures & $10(17 \%)$ \\
\hline & Increase Professional Education/Training & $6(10 \%)$ \\
\hline & Increase Funding & $4(7 \%)$ \\
\hline & Family Support & $3(5 \%)$ \\
\hline & Miscellaneous/No Comments & $1(2 \%)$ \\
\hline \multirow[t]{6}{*}{ Threats $(n=64)$} & Funding and Reimbursement & $23(36 \%)$ \\
\hline & Access to Services & $12(19 \%)$ \\
\hline & Infrastructure & $10(16 \%)$ \\
\hline & Miscellaneous/No Comment & $8(13 \%)$ \\
\hline & Professional Training/Skill & $6(9 \%)$ \\
\hline & Data/Document Sharing & $5(8 \%)$ \\
\hline
\end{tabular}

delay. However, most permanent hearing loss in infants and toddlers typically results in some degree of speech, language, or cognitive delay if appropriate early intervention services are not implemented (Moeller, 2000; Nicholas \& Geers, 2006). Although standardized questionnaires can assist in monitoring developmental growth, objective measures to determine specific levels of developmental delay in infants less than 12 months of age can be difficult to obtain. Therefore, most children with hearing loss qualify for Part C services under the condition that they are at risk due to "a diagnosed physical or mental condition that has a high probability of resulting in developmental delay" (White, 2007). EHDI coordinators reported that appropriate and clear 
Table 2. Top three themes for strengths

\begin{tabular}{ll}
\hline \multicolumn{1}{c}{ Themes } & \multicolumn{1}{c}{ Description } \\
\hline Effective Eligibility and Referral Process & All infants with any degree of hearing \\
& loss are eligible for early intervention \\
& services. \\
& Single point of entry. \\
& Strong protocols in place to ensure a \\
& seamless process into early intervention \\
& once identified with hearing loss. \\
Collaboration between Early Intervention & Strong advisory board. \\
and EHDI & Close relationship between EHDI and \\
& early intervention. \\
& Ongoing meetings to continue building \\
& relationships. \\
Adequate Infrastructure for Services & EHDI and early intervention are in the \\
& same division/department/agency. \\
& Early intervention (Part C) contracts with \\
& highly qualified providers to assist \\
& with appropriate service provisions. \\
& Legislation mandating services. \\
\hline
\end{tabular}

eligibility guidelines for serving children with hearing loss is a primary factor to successful implementation of effective early intervention services.

EHDI coordinators also identified good collaboration with a strong commitment to the EHDI process as an important aspect of effective early intervention services $(20 \%)$. When professionals across agencies are committed to a common goal and have a desire to develop positive interagency relationships, the impact can be significant. EHDI respondents indicated the importance of audiologists, physicians, and personnel in regional early intervention programs being sensitive to both the strengths and the needs of local early intervention agencies. Directly acknowledging potential turf issues, with open dialogue regarding specific agreements for service delivery, can maximize collaboration and serve to minimize negative outcomes. An associated component in developing good collaboration and a strong commitment to the EHDI system was the establishment of an effective advisory board. Having a mechanism for linking agencies can increase communication and help to represent the perspectives of all agencies involved in newborn screening and early intervention services.

Finally, $15 \%$ of the EHDI coordinators reported the value of having a strong infrastructure for providing statewide comprehensive services. Some states reported having contractual arrangements with specific audiology providers, resulting in better processes for referral and follow-up. The benefits of developing written policies and procedures across key agencies to ensure consistent 
and cohesive initial screening and to confirm enrollment in an early intervention program were also noted. Some state coordinators reported that the successful establishment of a data sharing system for increased communication and monitoring was critical to effective program function.

Another strength noted was strong program services (12\%), such as deaf education specialists providing the early intervention services, hearing aid loaner banks, offering several communication methodologies, data regarding the number of children with hearing loss served, and an early intervention program specific to serving children who are deaf and hard of hearing. Under the highly trained services providers responses (6\%), EHDI coordinators reported that their early interventionists had been trained in using materials from Learn to Talk Around the Clock (Rossi, 2003) and/or SKI HI curriculum (SKI-HI Institute, 2011), or that programs had good involvement from an NCHAM liaison and early interventionists trained in deaf education, speech-language pathology, or other related fields. Other strengths included quality improvement projects $(6 \%)$, electronic links for data sharing $(5 \%)$, family support programs (4\%), and funding (4\%).

\section{Weakness Characteristics}

The four most frequently cited weaknesses were poor communication/ coordination of services (23\%), no electronic link for data sharing $(19 \%)$, lack of appropriately trained personnel $(17 \%)$, and insufficient access to services $(17 \%)$ (Table 3). Twenty-three percent of EHDI coordinators described poor communication/coordination of services as a major program weakness, resulting in barriers to effective implementation of early intervention services. Respondents described a variety of breakdowns in service coordination, illustrating the challenges of agencies and professionals associated with EHDI programs. For example, EHDI coordinators reported that communication is compromised when EHDI agencies do not have a geographical link (e.g., programs housed in the same facility) or an electronic link through a shared database with key stakeholders. They reported that some children are lost to follow-up because of inadequate statewide EHDI communication policies and procedures for referral to Part $\mathrm{C}$ programs, when audiologists and physicians do not communicate with or make referrals to an early intervention agency, or when EHDI programs have no link with non-Part $C$ programs. Overall, communication breakdowns across the EHDI continuum of medical and audiological services can negatively impact prompt and effective early intervention services to children and families.

A primary program weakness for $19 \%$ of EHDI coordinators was not having an electronic link for data sharing across key agencies. EHDI coordinators stressed the importance of having a web-based or electronic database connection with either the state metabolic screening program, immunizations program, or the vital statistics/birth certificate registry. Without an electronic data 
Table 3. Top four themes for weaknesses

\begin{tabular}{|c|c|}
\hline Themes & Description \\
\hline $\begin{array}{l}\text { Poor Communication/Coordination of } \\
\text { Services }\end{array}$ & $\begin{array}{l}\text { Early intervention and EHDI are in } \\
\text { different divisions / departments / } \\
\text { agencies. } \\
\text { No single point of entry; inconsistent } \\
\text { services across state. } \\
\text { Early Intervention (Part C) will only try } \\
\text { for a limited amount of time to contact } \\
\text { family thus many children are lost in the } \\
\text { system. }\end{array}$ \\
\hline No Electronic Link for Data Sharing & $\begin{array}{l}\text { No linkage of data between EHDI and } \\
\text { early intervention services. } \\
\text { No "universal" referral form. } \\
\text { Only collect aggregate data making it } \\
\text { difficult to conduct follow-up activities. }\end{array}$ \\
\hline Lack of Appropriately Trained Personnel & $\begin{array}{l}\text { Lack of providers with expertise in } \\
\text { pediatric hearing loss. } \\
\text { Early interventionists are "generalists" } \\
\text { and have limited knowledge of hearing } \\
\text { loss and language development. } \\
\text { Lack of staff - no Part C coordinator, } \\
\text { limited deaf educators, audiologists, } \\
\text { early interventionists, EHDI staff for } \\
\text { follow-up services. }\end{array}$ \\
\hline Insufficient Access to Services & $\begin{array}{l}\text { Inconsistencies in Part C eligibility. } \\
\text { Insufficient program access in rural areas } \\
\text { of the state. } \\
\text { Incomplete information about all } \\
\text { communication options. }\end{array}$ \\
\hline
\end{tabular}

connection, babies are more likely to be lost to follow-up or experience a delay in hearing loss diagnosis. An electronic link for data tracking across agencies is believed to improve communication regarding referrals and results status, and could potentially improve the timeliness of early intervention referral.

The lack of appropriately trained personnel was reported by $17 \%$ of EHDI coordinators as a barrier to effective early intervention services. These coordinators described the negative impact of significant staff shortages across the state, including examples of having no Part $\mathrm{C}$ coordinator, insufficient numbers of early interventionists in Part $C$ programs, insufficient deaf mentor services, and too few pediatric audiologists. Furthermore, respondents reported compromised early intervention services because too many Part $C$ early intervention providers do not have specialized training related to hearing loss. Several EHDI coordinators noted that some early intervention providers 
were "generalists" with very little formal preservice preparation to develop appropriate goals and strategies for facilitating auditory learning in infants, toddlers, and preschoolers. In many states, it is the responsibility of the early interventionists to provide information to families about communication options. Insufficient knowledge and background to provide comprehensive, accurate, and unbiased information to families can result in inadequate service delivery.

Finally, insufficient access to comprehensive information and / or an inability to access services by families were also identified as weaknesses by $17 \%$ of EHDI coordinators. EHDI coordinators reported inconsistencies in Part $C$ eligibility, depending upon the family's geographical location and the eligibility regulations within a given region. Insufficient program access in rural areas of the state was commonly reported in this category, resulting in children and families who do not receive comprehensive or timely services. Furthermore, families in rural regions often do not receive complete information about all communication options with many families obtaining services that happen to be available, not necessarily the services best suited for their family.

Other themes identified were poor relationships among stakeholders (14\%) and funding $(6 \%)$. Lack of communication between early intervention and EHDI, "turf issues", lack of buy-in by professionals, no authority over the program, and significant internal conflict all contributed to poor relationships. Weaknesses identified under funding included lack of insurance coverage for services and sustainability of the early intervention program.

\section{Opportunities for EHDI Programs}

The three most frequently cited opportunities were improved relationships / service coordination (36\%), improved systems for electronic data sharing $(22 \%)$, and improved infrastructure and procedures (17\%) (Table 4). Reflection on the current status of service delivery and recognizing opportunities for program improvement can assist stakeholders in identifying potential shortterm and long-term program goals. Thirty-six percent of EHDI coordinators reported that the most important opportunity for improvement in early intervention services lies in identifying strategies to improve relationships and service coordination among stakeholders for comprehensive and timely early intervention services. Specifically, EHDI coordinators identified the value of key stakeholders meeting regularly with a focused agenda and a commitment to identifying barriers to families receiving appropriate services. Additional recommendations included having an active and committed advisory board to systematically address local and state needs. Furthermore, respondents indicated that identifying key "point persons" within the EHDI system to oversee specific aspects of service coordination can result in improved communication among both professionals and families. 
Table 4. Top three themes for opportunities

\begin{tabular}{|c|c|}
\hline Themes & Description \\
\hline $\begin{array}{l}\text { Improve Relationships/Service } \\
\text { Coordination }\end{array}$ & $\begin{array}{l}\text { Ongoing meetings and subcommittee } \\
\text { meetings between EHDI and Part C to } \\
\text { improve services. } \\
\text { Developed a network to increase } \\
\text { communication among related } \\
\text { organizations, agencies, and interested } \\
\text { parties. } \\
\text { Great leadership. }\end{array}$ \\
\hline $\begin{array}{l}\text { Improve Systems for Electronic Data } \\
\text { Sharing }\end{array}$ & $\begin{array}{l}\text { Linking data systems in at the state level. } \\
\text { New early intervention module in EHDI } \\
\text { data management system. } \\
\text { Incorporating Early Head Start into the } \\
\text { data management system. }\end{array}$ \\
\hline Improve Infrastructure and Procedures & $\begin{array}{l}\text { Strategic planning. } \\
\text { Defined protocols and guidelines. } \\
\text { Having legislation to support EHDI. }\end{array}$ \\
\hline
\end{tabular}

Twenty-two percent of respondents reported opportunities for improved services through establishing an electronic link for data sharing. A system for data exchange between EHDI coordinators, audiologists, and early interventionists to identify diagnostic results and early intervention enrollment was viewed as a fundamental factor in increasing the communication necessary to ensure access to early intervention services.

Similarly, $17 \%$ of EHDI respondents reported that future opportunities to improve the EHDI system for early intervention services involved improvement in infrastructure and operational procedures that addressed state and local practices. EHDI coordinators reported significant breakdowns in professionals making appropriate referrals and in ensuring appropriate follow-up services when written policies and procedures are insufficient, unclear, or are not being followed by all key EHDI service providers.

Other identified opportunities included increased professional education/ training $(10 \%)$, such as reaching out to teacher training programs, increasing communication with key stakeholders, and being involved with research. Increased funding (7\%) and family support (5\%) were also noted.

\section{Threats to EHDI Programs}

The three most frequently cited threats were funding and reimbursement $(36 \%)$, access to services (19\%), and infrastructure $(16 \%)$ (Table 5). Given the U.S. economic climate in 2010, it was not surprising to find that the most frequently cited threat to improved early intervention services was lack of 
Table 5. Top three themes for threats

\begin{tabular}{|c|c|}
\hline Themes & Description \\
\hline Funding and Reimbursement & $\begin{array}{l}\text { Budget cuts. } \\
\text { Insurance coverage issues. } \\
\text { Service providers are withdrawing contracts to } \\
\quad \text { provide early intervention services due to funding. }\end{array}$ \\
\hline Access to Services & $\begin{array}{l}\text { Geographic issues with large rural population. } \\
\text { Families go out of state to access services (i.e., no } \\
\text { cochlear implant team in state). } \\
\text { Family issues (i.e., move, do not respond to } \\
\text { attempted contacts, do not permit home visits). }\end{array}$ \\
\hline Infrastructure & $\begin{array}{l}\text { Lack of legislative support. } \\
\text { Difficult to track children and their families due to } \\
\text { the various components of EHDI being spread } \\
\text { across different agencies / divisions / departments. } \\
\text { Methodology/language controversy making it } \\
\text { difficult to move forward (i.e., American Sign } \\
\text { Language, listening and spoken language, Cued } \\
\text { Speech, etc.). }\end{array}$ \\
\hline
\end{tabular}

funding. Thirty-six percent of EHDI coordinators cited concerns related to funding and reimbursement as a primary barrier to ensuring effective implementation of early intervention services to all families, regardless of where the family lives and which communication option is chosen. EHDI coordinators overwhelmingly stated that sufficient funding to hire and train staff was a primary threat to effective early intervention services. Respondents expressed concern that budget cuts have led to increased caseloads for Part $C$ providers, which could result in a reduction of available services.

The second most frequently cited threat involved concerns that too many families are unable to access needed services. Nineteen percent of EHDI coordinators expressed concern that families living in rural areas of the state do not receive the services they need to support their chosen method of communication. EHDI respondents reported that some families have moved to other states to obtain services for cochlear implant mapping or to access educational services that specialize in the family's communication methodology of choice, such as a listening and spoken language or a bilingual-bicultural approach.

Finally, $16 \%$ of EHDI coordinators expressed the need for improved infrastructure, particularly in the form of established and clearly written policies that are understood and accepted by key stakeholders who serve children with hearing loss. Some EHDI coordinators reported challenges when services are provided by separate agencies, especially when there are ineffective systems for communicating patient demographic information and test results. This information is vital to ensure continuity and timeliness of service delivery while 
adhering to confidentiality laws and policies. State legislative support was a reported threat to EHDI infrastructure, particularly when states do not mandate that hearing screening results be reported to the state EHDI coordinator.

Two additional threats included professional training and skills (9\%), such as difficulty recruiting appropriate professionals and staff, and data/documentation sharing $(8 \%)$.

\section{TOWS Analysis Matrix}

By applying the TOWS analysis to themes identified in the SWOT analysis, specific recommendations, or "strategic options," were derived from the internal and external environmental factors identified by the EHDI coordinators. By systematically pairing the four categories of the SWOT analysis, recommendations can be derived to maximize resources and effect positive change. Based on the SWOT themes, 8 individual recommendations were made regarding early intervention services (see Table 6). To maximize opportunities, addressing strengths can maximize efficiency and lead to positive change (S-O strategy). For example, service coordination can be increased when EHDI coordinators and Part C providers evaluate their state's written plan, policies, and procedures to ensure that the state has an appropriate and effective Part $C$ eligibility and referral process. To overcome weaknesses, opportunities must be pursued (W-O strategy). For example, critique of the written plan among key stakeholders with the goal of establishing program priorities, short-term and long-term goals, and strategies to improve professional relationships can minimize miscommunication among EHDI service providers and reduce the potential for a missed referral for early intervention services or a diagnostic evaluation. Identifying ways to use strengths will ultimately reduce vulnerability to threats (S-T strategy). For example, EHDI service providers who rarely communicate with other EHDI colleagues may be less able to effect change in funding and reimbursement. A collaborative approach among EHDI service providers, such as establishing a state coalition of professionals from key agencies, may be an effective mechanism to identify funding needs and barriers and to determine a proactive plan to improve funding opportunities. Finally, establishing defensive plans will help prevent weaknesses from making programs and services susceptible to threats (W-T strategy). For example, EHDI coordinators who reported program weaknesses due to poor electronic data tracking systems might identify specific areas of data reporting vulnerability, and develop written recommendations and an action plan for improving this aspect of EHDI services. Identifying the program needs and developing a specific action plan can ultimately minimize the program weakness, therefore minimizing the threats to the EHDI system. Out of these 8 recommendations, there were 3 distinct strategies that emerged to help states develop effective early intervention programs. Specifically, states are encouraged to: 1) establish an appropriate 
Table 6. TOWS analysis matrix for early intervention

\begin{tabular}{|c|c|c|c|}
\hline & \multicolumn{2}{|c|}{ Internal } \\
\hline & & Strengths & Weaknesses \\
\hline L & 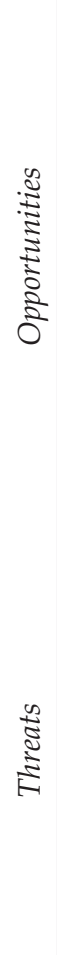 & $\begin{array}{l}\text { S-O Strategies } \\
\text { 1. EHDI and Part C coordinators } \\
\text { evaluate their state's written } \\
\text { plan for service provision to } \\
\text { ensure that each state has an } \\
\text { appropriate and effective } \\
\text { Part C eligibility and referral } \\
\text { process. } \\
\text { 2. Identify strategies for } \\
\text { improving communication, } \\
\text { such as written policies and } \\
\text { procedures or development of } \\
\text { an active advisory board. } \\
\text { S-T Strategies } \\
\text { 1. Establish a state coalition of } \\
\text { professionals from key agencies } \\
\text { who can identify funding needs, } \\
\text { barriers, and recommendations. } \\
\text { 2. Evaluate current service delivery } \\
\text { models to ensure all families } \\
\text { have access to comprehensive } \\
\text { information and appropriate } \\
\text { services, even in rural locations. }\end{array}$ & $\begin{array}{l}\text { W-T Strategies } \\
\text { 1. Identify the current system of } \\
\text { data sharing among } \\
\text { stakeholders and develop } \\
\text { written recommendations and } \\
\text { action plan for improving } \\
\text { electronic data tracking. } \\
\text { 2. Identify opportunities for } \\
\text { agencies to collaborate the } \\
\text { expertise and resources of their } \\
\text { personnel for increased training. }\end{array}$ \\
\hline
\end{tabular}

$\mathrm{S}=$ Strength $\mathrm{W}=$ Weakness $\mathrm{O}=$ Opportunity $\mathrm{T}=$ Threat

and effective eligibility and referral process for entry into early intervention; 2) establish a state advisory board or coalition group to identify funding needs and barriers; and 3) make recommendations to improve data management systems and reporting of data to key stakeholders.

\section{Limitations}

Limitations of this study include those inherent in the application of SWOT/ TOWS analysis when applied to rapidly changing conditions. SWOT analysis is a subjective tool that explores conditions that are perceived rather than objectively measured. In this sense, the SWOT analysis is influenced by the perspectives of those who completed the surveys as well as those who analyzed the responses. Furthermore, SWOT analysis characterizes the situation 
at a specific point in time. In addition, SWOT analysis, as applied here, resulted in overlapping themes within a category. For example, funding and data sharing could each be identified as a strength, weakness, opportunity, or threat. (see White \& Blaiser, 2011, for additional information regarding the selection of SWOT/TOWS for this study and further comment regarding interpretation and application of findings).

\section{Summary}

Implementation of newborn hearing screening programs and early identification of hearing loss in children has been highly successful over the past two decades. Unfortunately, for many families of children with hearing loss, implementation of effective and timely early intervention services has lagged behind other EHDI areas (White, 2007). EHDI coordinators provided compelling data to indicate that specific fundamental improvements in communication, policies, and written procedures among key stakeholders would make a significant difference to families and service providers. Assurance that each state plan supports an effective early intervention eligibility and referral process was stressed by EHDI coordinators as a fundamental aspect of program strength. However, the mere existence of state early intervention services is not adequate if families are not effectively integrated into active and successful early intervention programs. The value of effective communication among key EHDI stakeholders, such as physicians, audiologists, and early intervention providers, was highlighted in the survey results as a critical component to EHDI services. The commitment to a common goal and the desire to develop strong collegial relationships can be the prompting factor for implementing positive changes across a variety of program needs. The benefits of early intervention services are evidenced by the many children who have developed age-appropriate speech and language skills and who have shown cognitive and academic achievement consistent with that of their same-aged peers without hearing loss.

Stakeholders in EHDI systems are faced with challenging barriers that include an economic downturn requiring more services to be provided with fewer resources in a limited time period. Learning to identify successful methods and strategies for overcoming the challenges faced on a daily basis can assist continued forward momentum for service improvement. The desire to provide excellent early intervention services to children and families and to witness success in each child being served is a goal of all professionals in the EHDI system. Working together, progress in service delivery for children with hearing loss and their families can be achieved.

\section{Acknowledgement}

The work reported in this article was funded in part by the Maternal and Child Health Bureau under Cooperative Agreement \# U52MC04391 with 
the National Center for Hearing Assessment and Management at Utah State University. The opinions expressed in the article are those of the authors and do not necessarily reflect those of the Bureau.

\section{References}

Centers for Disease Control and Prevention (CDC). (2008). Early hearing detection and intervention hearing screening and follow-up survey. Retrieved May 19, 2011, from http:/ / www.cdc.gov/ncbddd/ehdi/data.htm.

Centers for Disease Control and Prevention (CDC). (2010a). Annual data. Early hearing detection and intervention (EHDI) program. Retrieved May 17, 2011, from http://www.cdc.gov/ncbddd/hearingloss/ehdi-data.html.

Centers for Disease Control and Prevention (CDC). (2010b). Early hearing detection and intervention (EHDI) national goals. Retrieved May 17, 2011, from http://www.cdc.gov/ncbddd/hearingloss/ehdi-goals.html.

Hands \& Voices. (2010). Early identification and intervention: The journey starts here. Retrieved May 24, 2011, from http://www.handsandvoices.org/arti cles/early_intervention/early_id_journey.html.

Hager, J. H., \& Giannini, M. (2006). United States Department of Education, Office of Special Education and Rehabilitation Services. Part C Coordinators. Retrieved May 24, 2011, from http:/ / www.infanthearing.org/earlyintervention/HagerGianninni\%20letter.pdf.

Joint Committee on Infant Hearing (JCIH). (2007). Year 2007 position statement: Principles and guidelines for early hearing detection and intervention programs. Pediatrics, 120(4), 898-921

Kennedy, C. R., McCann, D. C., Campbell, M. J., Law, C. M., Mullee, M., Petrou, S., et al. (2006). Language ability after early detection of permanent childhood hearing impairment. New England Journal of Medicine, 354(20), 2131-2141.

Marge, D. K., \& Marge, M. (2005). Beyond newborn hearing screenings: Meeting the educational and healthcare needs of infants and young children with hearing loss in America. Report of the National Consensus Conference on Effective Educational and Health Care Interventions for Infants and Young Children with Hearing Loss, September 10-12, 2004, Syracuse, NY.

Moeller, M. P. (2000). Early intervention and language development in children who are deaf and hard of hearing. Pediatrics, 106(3), E43.

National Center for Hearing Assessment and Management (2010). Early intervention. Retrieved May 24, 2011, from http://www.infanthearing.org/ear lyintervention/index.html.

National Dissemination Center for Children with Disabilities. (2010). Overview of early intervention. Retrieved April 27, 2011, from http://nichcy.org/babies/ overview.

Nicholas, J. G., \& Geers, A.E. (2006). Effects of early experience on the spoken language of deaf children at 3 years of age. Ear \& Hearing, 27(3), 286-98. 
Rossi, K. (2003). Learn to talk around the clock: An early interventionists toolkit. Washington, DC: Alexander Graham Bell Association for the Deaf and Hard of Hearing.

SKI-HI Institute. (2011). Project SKY-HI. Retrieved June 13, 2011, from http://www.skihi.org/SkihiMod.html.

U.S. Department of Health and Human Services (DHHS). (1990). Healthy People 2000: National health promotion and disease prevention objectives. Washington, DC: Public Health Service.

White, K. R. (2004). Early hearing detection and intervention programs: Opportunities for genetic services. American Journal of Medical Genetics, 130(A), 29-36.

White, K. R. (2007). Early intervention for children with permanent hearing loss: Finishing the EHDI revolution. The Volta Review, 106(3), 237-258.

White, K. R., Forsman, I., Eichwald, J., \& Munoz, K. (2010). The evolution of Early Hearing Detection and Intervention programs in the United States. Seminars in Perinatology, 34(2), 170-179.

White, K., \& Blaiser, K. (2011). Strategic planning to improve early hearing detection and intervention programs. The Volta Review, 111(2), 83-108.

Yoshinaga-Itano, C. (2003). Early intervention after universal neonatal hearing screening: Impact on outcomes. Mental Retardation and Developmental Disabilities Research Reviews, 9, 252-266.

Yoshinaga-Itano, C. \& Gravel, J. S. (2001). The evidence for universal hearing screening. American Journal of Audiology, 10(2), 62-63 D.

Yoshinaga-Itano, C., Sedey, A. L., Coutler, D. K., \& Mehl, A. L. (1998). Language of early-and later-identified children with hearing loss. Pediatrics, 102, 1161-1171. 

The Volta Review, Volume 111(2), Summer 2011, 151-164

\title{
Integrating the Medical Home into the EHDI Process
}

\author{
Karen F. Muñoz, Ed.D., CCC-A; Lauri Nelson, Ph.D.; \\ Tamala S. Bradham, Ph.D., CCC-A; Jeff Hoffman, M.S., CCC-A; and \\ K. Todd Houston, Ph.D., CCC-SLP, LSLS Cert. AVT
}

State coordinators of early hearing detection and intervention (EHDI) programs completed a strengths, weaknesses, opportunities, and threats, or SWOT, analysis that examined 12 areas within state EHDI programs. Related to how the medical home is integrated into the EHDI process, 273 items were listed by 48 coordinators, and themes were identified within each SWOT category. A threats, opportunities, weaknesses, and strengths, or TOWS, analysis yielded 12 recommendations that focused on similar themes. Out of these 12 recommendations, 3 distinct strategies emerged. Specifically, states should: 1) utilize chapter champions to expand educational outreach to other health care providers, primary care physicians, medical homes, and residency training programs; 2) recruit several chapter champions to represent different areas within medicine as well as across the state; and 3) improve data management systems to allow for easier reporting and to alert the medical home/primary care provider about newborn hearing test results. Addressed appropriately, these recommendations can significantly strengthen medical home integration in state EHDI programs.

\section{Introduction}

The importance of early identification of hearing loss has long been recognized (JCIH, 1971), although it has only been in the past two decades that

Karen F. Muñoz, Ed.D., CCC-A, is an Assistant Professor in the Department of Communicative Disorders and Deaf Education at Utah State University. Lauri Nelson, Ph.D., is an Assistant Professor in the Department of Communicative Disorders and Deaf Education at Utah State University. Tamala S. Bradham, Ph.D., CCC-A, is an Assistant Professor in the School of Medicine, Department of Hearing and Speech Sciences, at Vanderbilt University. Jeff Hoffman, M.S., CCC-A, is a Senior Research Scientist - Audiologist in the National Center for Hearing Assessment and Management at Utah State University. K. Todd Houston, Ph.D., CCC-SLP, LSLS Cert. AVT, was an Assistant Professor in the Department of Communicative Disorders and Deaf Education at Utah State University at the time of this study and is currently at The University of Akron. Correspondence concerning this manuscript should be directed to Dr. Muñozat karen.munoz@usu.edu. 
identification of congenital hearing loss through newborn hearing screening (NHS) has become a standard of care. Currently, there are 43 U.S. states and territories that mandate NHS (National Center for Hearing Assessment and Management [NCHAM], 2010). The early identification of infant hearing loss has significant advantages for expressive and receptive speech and language development. Research has shown that through early identification, appropriate use of hearing technology, and family-centered early intervention services with properly-trained professionals, children with hearing loss experience better language, speech, cognitive, and social-emotional development than later-identified children who did not receive early intervention services (Kennedy, et. al., 2006; Moeller, 2000; Yoshinaga-Itano, Sedey, Coulter, \& Mehl, 1998). The EHDI process is a relatively new system of care and challenges exist in connecting families to needed services in a timely manner, such as connecting families to hearing assessment services if the child does not pass the initial NHS.

Primary health care providers, in partnership with other professionals, play a pivotal role in creating a medical home to ensure that children referred from NHS receive needed follow-up care. As defined by the American Academy of Pediatrics (AAP), the ideal medical home should be accessible, familycentered, continuous, comprehensive, coordinated, compassionate, and culturally effective (AAP, 2002). In the medical home model, the physician is a case manager responsible for the ongoing care of the child. For children born at home or not screened at birth for other reasons, physicians need to know where to refer the child so the screening can be completed (Muñoz, Shisler, Moeller, \& White, 2009). According to the Joint Committee on Infant Hearing (JCIH) guidelines, screening should be completed before 1 month of age $(\mathrm{JCIH}$, 2007). Additionally, there are conditions that place a child at risk for progressive or late-onset hearing loss, and screening during early childhood is particularly important for children who exhibit one or more of the risk indicators for hearing loss (JCIH, 2007). Therefore, ongoing surveillance of hearing is needed for all children. For infants who pass NHS, the JCIH states that physicians should provide regular surveillance during periodic well-child visits at specified intervals, or anytime the health care professional or family has a concern (JCIH, 2007).

Even though congenital hearing loss occurs more frequently than any other condition for which NHS is available, it is still a relatively lowincidence condition, affecting about 3 out of every 1,000 newborns (White, 2007). Thus, most physicians will serve only a small number of infants with hearing loss during his or her career. Added to that, the field of early intervention has undergone dramatic changes over the past two decades (White, Forsman, Eichwald, \& Muñoz, 2010). This has made it difficult for physicians and other health care providers to stay up-to-date regarding best practices for infants and young children with permanent hearing loss. 
Primary care physicians will be best prepared to support families of infants and young children with suspected or confirmed hearing loss if they understand the critical components of hearing screening, diagnosis, and intervention as well as the time-sensitive nature of these services. Families are particularly at risk for being lost to follow-up after referral from NHS. When an infant does not pass the NHS, the infant's primary health care provider should be notified about the need for follow-up screening and/or audiological testing. Unfortunately, physicians may assume that no news is good news as it relates to test results. This is not a safe assumption because breakdowns in communication are common. Even when there is an intent to notify the primary health care provider, it may not occur for various reasons - the physician of record at the hospital may not be the one who actually provides care for the infant, parents may not continue with the same physician who cared for the baby in the hospital, or families may access a birthing hospital in a nearby state rather than their home state, thereby complicating the communication of results from the state EHDI office to the physician in their home state. For physicians to provide appropriate guidance to families following a failed hearing screening, physicians need evidence-based information on the audiological test battery in order to refer families to audiologists that are knowledgeable about infant testing and have the equipment necessary to complete a comprehensive assessment (Harlor \& Bower, 2009; Muñoz \& Nelson, 2010).

Once hearing loss has been identified, the referral and case management process becomes more complex and the physician's need for information increases. When hearing loss is diagnosed, the primary health care provider should confirm that referrals are being made to identify the etiology of the hearing loss, to determine if other co-existing conditions exist that need to be considered for follow-up care, and to make recommendations for medical, audiological, and educational intervention. According to the JCIH (2007), infants and toddlers with permanent hearing loss should be referred to an otolaryngologist who has specific expertise in pediatric hearing loss management. This includes performing a medical assessment and making referrals for additional medical evaluations, such as a referral to a geneticist and an ophthalmologist, as well as providing medical clearance for amplification if parents choose to pursue use of hearing technology with their child. Parents have an overwhelming need for information when a hearing loss is diagnosed and often turn to their physician for support. Physicians need a basic understanding of the issues and where to direct families for complete and unbiased guidance.

Recently, critical gaps in physician attitudes, practices, and knowledge about EHDI were documented in focus group findings (Moeller, Eiten, White, \& Shisler, 2006) and through a national survey to which 1,968 physicians, consisting primarily of pediatricians and family practitioners, responded (Moeller, White, \& Shisler, 2006). The survey results indicated that only $12 \%$ of pediatricians and $17 \%$ of family practitioners received screening results on more than $50 \%$ of their patients (Moeller, White, et al., 2006). Furthermore, when a hearing 
loss is identified, only $14 \%$ of respondents reported that their medical training program had adequately prepared them to meet the needs of infants with permanent hearing loss (Moeller, White, et al., 2006). Follow-up medical care and referrals was another area of weakness, and the need for a referral to a geneticist was recognized by only $11 \%$ of pediatricians, $3 \%$ of family practitioners, and $22 \%$ of otolaryngologists. Furthermore, the need to refer to an ophthalmologist was recognized by only $1 \%$ of pediatricians, $0 \%$ of family practitioners, and $7 \%$ of otolaryngologists. In relation to hearing aid fitting, only $53 \%$ of the survey respondents knew that infants who were 3 months of age or younger could be fitted with hearing aids, and $18 \%$ thought it was necessary to wait until the child was 12 months of age or older. Additionally, almost $50 \%$ of survey respondents were incorrect about the type and level of hearing loss that would warrant a referral for a cochlear implant evaluation. Almost 70\% reported a lack of confidence in discussing this surgical procedure with families of such children, and $57 \%$ said they were "Not Confident" in talking with parents about the "use of sign language versus auditory/oral communication."

Recognizing the need to educate physicians, and as NHS was becoming a standard of care, in 2001 the AAP identified EHDI Chapter Champions ${ }^{1}$ for each state. The Chapter Champions are members of state AAP chapters and serve as a resource to physicians on EHDI issues. They also provide educational outreach to physicians to improve physician knowledge and involvement in the EHDI process. Chapter Champions provide support in a variety of ways, such as through dissemination of information via state newsletters, pediatric grand rounds, and service on state NHS advisory boards. The AAP also disseminates a monthly EHDI e-newsletter highlighting current topics and events of interest related to EHDI.

Identification of the common challenges faced by EHDI programs related to the medical home for young children with hearing loss can provide valuable information to improve program effectiveness and lead to better outcomes for infants who do not pass the newborn hearing screening. The purpose of this study was to complete a systematic review using a strengths, weaknesses, opportunities, and threats, or SWOT, and a threats, opportunities, weaknesses, and strengths, or TOWS, analysis to determine the current EHDI practices in medical homes, and to identify recommendations to improve program effectiveness that lead to better outcomes for children with hearing loss.

\section{Methods}

Fifty-one EHDI coordinators were asked to complete an online survey. Related to medical home integration, the survey asked EHDI coordinators to report at

\footnotetext{
${ }^{1}$ For more information about the AAP EHDI Chapter Champions program, visit www.medicalhomeinfo.org/how/clinical_care/hearing_screening/ehdi.aspx.
} 
least 1 strength, weakness, opportunity, and threat for use in strategic planning. The responses obtained were reviewed by a panel of experts and categorized into common themes within four strategic planning areas. To generate recommendations from the SWOT analysis, a TOWS matrix was used to match identified strengths with opportunities (S-O strategy), strengths with threats (S-T strategy), weaknesses with opportunities (W-O strategy), and weaknesses with threats (W-T strategy). For an in-depth review of the SWOT/TOWS analysis and its use in the current study, the reader is referred to White and Blaiser, 2011.

\section{Results}

Forty-eight EHDI coordinators (94\%) completed the medical home integration section of the survey. The respondents identified 78 items in the strength category, 64 items in the weaknesses category, 61 items in the opportunities category, and 70 items in the threats category for a total of 273 responses. Based on these responses, several specific themes were generated for each category: 9 for strengths, 8 for weaknesses, 8 for opportunities, and 8 for threats (Table 1). Each category also had a miscellaneous section that included no comment, N/A, or items that did not fit within the themes identified.

\section{Strength Characteristics}

The three most frequently cited strengths were Chapter Champions (17\%), system support (16\%), and education (14\%) (Table 2). Having an AAP Chapter Champion was the most frequently reported strength for integrating the medical home into the EHDI process. The specific element that made the champion a strength included having someone who is supportive, enthusiastic, and committed. It was also mentioned that it was important to have the chapter champion on the state EHDI advisory board. System support for EHDI was another frequently mentioned strength, specifically having a strong medical home initiative in the state as well as commitment to the medical home concept. Education was also a strength when there were mechanisms in place for physician education regarding their role in EHDI, including sending routine educational packets to physicians when a hearing loss is identified and implementing awareness activities, such as newsletters and conference materials.

Identification of the primary care physician (13\%) was noted as a strength, specifically when the physician is identified at the hospital or when there is a mechanism in place to assist with linking families to a primary care physician. Communication (13\%) was another strength, such as when hospitals and audiologists support the primary care physician in achieving timely follow-up. Other related strengths included notifying the primary care physician 
Table I. Frequency of themes within each SWOT category $(n=273)$

\begin{tabular}{|c|c|c|}
\hline SWOT category & Themes & Frequency $n(\%)$ \\
\hline Strengths $(n=78)$ & $\begin{array}{l}\text { Chapter Champion } \\
\text { System Support } \\
\text { Education } \\
\text { Identification of the PCP } \\
\text { Communication } \\
\text { Notifying PCP of the Results } \\
\text { Collaboration/Relationships } \\
\text { Miscellaneous/No Comments } \\
\text { Data System }\end{array}$ & $\begin{array}{l}12(17 \%) \\
11(16 \%) \\
10(14 \%) \\
9(13 \%) \\
9(13 \%) \\
9(13 \%) \\
9(13 \%) \\
5(7 \%) \\
4(6 \%)\end{array}$ \\
\hline Weaknesses $(n=64)$ & $\begin{array}{l}\text { Education } \\
\text { Data System } \\
\text { Access } \\
\text { System Support } \\
\text { Identification of the PCP } \\
\text { Miscellaneous/No Comments } \\
\text { Communication } \\
\text { Timeliness of Referral }\end{array}$ & $\begin{array}{c}18(28 \%) \\
9(14 \%) \\
9(14 \%) \\
8(12 \%) \\
7(11 \%) \\
6(10 \%) \\
4(6 \%) \\
3(5 \%)\end{array}$ \\
\hline Opportunities $(\mathrm{n}=61)$ & $\begin{array}{l}\text { Education } \\
\text { Data System } \\
\text { Chapter Champion } \\
\text { Communication } \\
\text { System Support } \\
\text { Miscellaneous/No Comments } \\
\text { Funding } \\
\text { Legislation }\end{array}$ & $\begin{array}{c}18(30 \%) \\
8(13 \%) \\
8(13 \%) \\
6(10 \%) \\
6(10 \%) \\
6(10 \%) \\
5(8 \%) \\
4(6 \%)\end{array}$ \\
\hline Threats $(\mathrm{n}=70)$ & $\begin{array}{l}\text { Commitment } \\
\text { Access } \\
\text { Miscellaneous/No Comments } \\
\text { Funding } \\
\text { System Support } \\
\text { Communication } \\
\text { Education } \\
\text { Reporting }\end{array}$ & $\begin{array}{l}15(21 \%) \\
14(20 \%) \\
14(20 \%) \\
8(11 \%) \\
6(9 \%) \\
5(7 \%) \\
5(7 \%) \\
3(4 \%)\end{array}$ \\
\hline
\end{tabular}

of the screening results $(13 \%)$, collaboration $(13 \%)$, and implementing data reporting and tracking systems that allow the physician to access results for NHS (6\%).

\section{Weakness Characteristics}

The three most frequently cited weaknesses were education (28\%), data systems (14\%), and access (14\%) (Table 3). Education was the most frequently reported weakness and the specific elements that made it a weakness included the need for a coordinated plan to provide "just in time" education. Better education is 
Table 2. Top three themes for strengths

\begin{tabular}{lc}
\hline \multicolumn{1}{c}{ Themes } & Description \\
\hline AAP EHDI Chapter Champion & Active Chapter Champion(s) with standing \\
& meetings and conference calls. \\
& Assists with educating other physicians. \\
& Have several Chapter Champions. \\
System Support & Medical home concept integrated into the \\
& medical community. \\
& Complete buy-in/commitment by physicians \\
& and state programs. \\
Education & Emphasize medical home concept in residency \\
& training program. \\
& Publish articles in physician newsletters. \\
& Present at physician conferences. \\
\hline
\end{tabular}

needed to inform physicians about hearing loss, EHDI guidelines, appropriate referrals to other professionals, specific testing needs, ongoing monitoring, and the medical home concept. Data reporting and tracking systems were also a weakness, specifically when reporting is not mandated. For example, physicians often do not report the results of NHS performed in their office to the state EHDI program and not all primary care physicians use the state data system, resulting in incomplete data. Access was another frequently cited weakness, particularly when families do not have access to a medical home or are uninsured, and when there are not enough primary care physicians available.

System support (12\%) was also a weakness, primarily when EHDI is not supported by the state pediatric association, when there is not an AAP Chapter Champion, when there is a lack of involvement in the state EHDI program,

Table 3. Top three themes for weaknesses

\begin{tabular}{cl}
\hline \multicolumn{1}{c}{ Themes } & \multicolumn{1}{c}{ Description } \\
\hline Education & $\begin{array}{l}\text { Lack of knowledge by physicians about hearing loss and EHDI. } \\
\text { Solo or rural provider practices do not have up-to-date information. } \\
\text { Lack of parent and provider knowledge about medical homes. }\end{array}$ \\
Data System & $\begin{array}{l}\text { Data management system is not linked to medical homes / physicians. } \\
\text { Cannot track those lost to follow-up. } \\
\text { No mandate to report data. }\end{array}$ \\
& $\begin{array}{l}\text { Families are not able to access medical homes. } \\
\text { Shortage of physicians/primary care physicians. } \\
\text { Lack of insurance coverage. }\end{array}$ \\
\hline
\end{tabular}


and when there is a lack of an established medical home effort in the state. Identification of the primary care physician (11\%) was a weakness when not all parents identified a physician, an incorrect physician is listed, and, as some EHDI coordinators indicated, the difficulty of the community care system in Medicaid to identify a primary care physician prior to discharge. Communication $(6 \%)$ was a weakness for some states, such as when physicians do not return phone calls, as was timeliness of referral (5\%), specifically when physicians require multiple visits before referring and delayed referrals to diagnostic testing.

\section{Opportunities for EHDI Programs}

Education (30\%), data systems (13\%), and chapter champions (13\%) were the three most frequently cited opportunities (Table 4). Education was the most frequently reported opportunity and the specific elements that made it an opportunity included presentations at pediatric grand rounds, working with pediatric residents, distributing mailings and newsletters, using modern technology for physician education in rural areas of the state, and having an audiology consultant committee dedicated to enhancing physician education. Data reporting and tracking systems were also a frequently reported opportunity, and the specific elements that made it an opportunity included the ability to generate risk factor reports, integration of the primary care physician into an online data management system, and inclusion of the NHS results in the immunization data system. The AAP Chapter Champion was another opportunity, specifically having support from the Chapter Champion, adding a second Chapter Champion, and having the Chapter Champion on the NHS advisory committee.

Communication $(10 \%)$ was another opportunity, specifically networking with the primary care physician to reduce loss to follow-up and improving communication by having more than one physician on the NHS advisory committee. Respondents noted system support (10\%) as an opportunity,

Table 4. Top three themes for opportunities

\begin{tabular}{|c|c|}
\hline Themes & Description \\
\hline Education & $\begin{array}{l}\text { Present at Pediatric Grand Rounds at Hospital. } \\
\text { Developing a training module for residents. } \\
\text { Mailings and newsletters about EHDI and it's role in } \\
\text { medical homes. }\end{array}$ \\
\hline Data System & $\begin{array}{l}\text { Obtaining a new data management system. } \\
\text { Linking medical homes to the online EHDI data } \\
\text { management system. } \\
\text { Creating a primary care physician view of hearing screening/ } \\
\text { test results in the new data management system. }\end{array}$ \\
\hline AAP Chapter Champion & Adding additional Chapter Champions. \\
\hline
\end{tabular}


primarily when there is a state level commitment to the medical home and new medical home initiatives. Funding $(8 \%)$ is an opportunity when local funding is identified (specific use of funding was not specified), funding is increased from the Centers for Disease Control and Prevention (CDC) for data tracking systems, and funding is received from Maternal and Child Health Bureau (MCHB) to replicate National Initiative for Children's Healthcare Quality (NICHQ) activities to reduce loss to follow-up. Legislation (6\%) was also mentioned by a few states as an opportunity, specifically to require a primary care physician to be on the NHS advisory board.

\section{Threats to EHDI Programs}

The three most frequently cited threats were commitment $(21 \%)$, access $(20 \%)$, and funding (11\%) (Table 5). Commitment to EHDI was the most frequently reported threat and the specific elements that made it a threat were based on the concern that since physicians see few children with hearing loss it can be difficult to engage some of them in the process for follow-up. The commitment to EHDI can be threatened by lack of physician interest if family practice physician associations are not engaged with EHDI, if there is no Chapter Champion, and if a physician advises parents to "wait and see" rather than refer for testing. Access was also a frequently reported threat to EHDI programs and the specific elements that made it a threat included the barriers to parents having a medical home (e.g., socioeconomic, culture, transient population), transportation problems, limited physician time, and changes in the medical home. Funding was an area reported as a threat as it relates to reimbursement, limited resources, uninsured children, and fewer professional educational opportunities due to budget cuts.

Table 5. Top three themes for threats

\begin{tabular}{|c|c|}
\hline Themes & Description \\
\hline Commitment & $\begin{array}{l}\text { Too many primary care providers advise the "wait and see" approach. } \\
\text { Lack of physician interest/not engaged. } \\
\text { Busy professionals who don't accept "new" ways. }\end{array}$ \\
\hline Access & $\begin{array}{l}\text { Families lack of transportation to medical homes/Families change } \\
\text { medical homes. } \\
\text { Lack of specialists, primary care physicians; primary care } \\
\text { physicians are overwhelmed, limited time. } \\
\text { No centralized referral resource system. }\end{array}$ \\
\hline Funding & $\begin{array}{l}\text { Reimbursement for services are poor. } \\
\text { State funding is unstable. } \\
\text { Cuts in health care. } \\
\text { Uninsured children. }\end{array}$ \\
\hline
\end{tabular}


Other threats included system support (9\%) and communication (7\%), specifically when it is difficult to reach all providers and obtain opportunities to speak at physician meetings and when facing travel restrictions. Education $(7 \%)$ was another threat, primarily due to the lack of physician education about EHDI and physicians' limited exposure to hearing loss. Reporting (4\%) was also noted as a threat due to noncompliance of providers in sending reports to the medical home, and primary care physicians not reporting results of screenings performed in their office to the EHDI program.

\section{TOWS Analysis Matrix}

By applying the TOWS analysis matrix to the themes identified in the SWOT analysis, specific recommendations, or "strategic options," were derived from the internal and external environmental factors identified by the EHDI coordinators. By systematically pairing the four categories of the SWOT analysis, recommendations can be derived to maximize resources and effect positive change. Based on the SWOT themes, 12 individual recommendations were made (Table 6). To maximize opportunities, identification of strengths can lead to positive change (S-O strategy). For example, by utilizing Chapter Champions at the state level there may be opportunities for conversations to occur on how to educate physicians and increase access to care. By pursuing opportunities, weaknesses can be minimized (W-O strategy). For example, recruitment of additional Chapter Champions can provide the opportunity to reach more physicians in the state. Also, sharing data from the state data management system can improve physician understanding about the components of the EHDI system and lead to improved patient care. Identification of ways to use strengths can ultimately reduce vulnerability to threats (S-T strategy). For example, providing primary care physicians with EHDI data can increase their understanding of the importance of timely service delivery and help them recognize if patients in their practice are receiving services within JCIH 2007 quality benchmarks. Finally, establishing defensive plans will help to prevent weaknesses from making programs susceptible to threats (W-T strategy). For example, to effect change regarding access to care, funding and reimbursement, and education, states need strong data management systems to report their findings and outcomes. Out of these 12 recommendations, 3 distinct strategies emerged for integrating the medical home into the EHDI system emerged. Specifically, states should 1) use their AAP EHDI Chapter Champions to expand educational outreach to other health care providers, primary care physicians, medical homes, and residents in training; 2) recruit several Chapter Champions to represent different areas within medicine as well as across their state; and 3) improve their data management system to allow for easier reporting and to alert the medical home/primary care provider about newborn hearing test results. 
Table 6. TOWS analysis matrix for medical homes

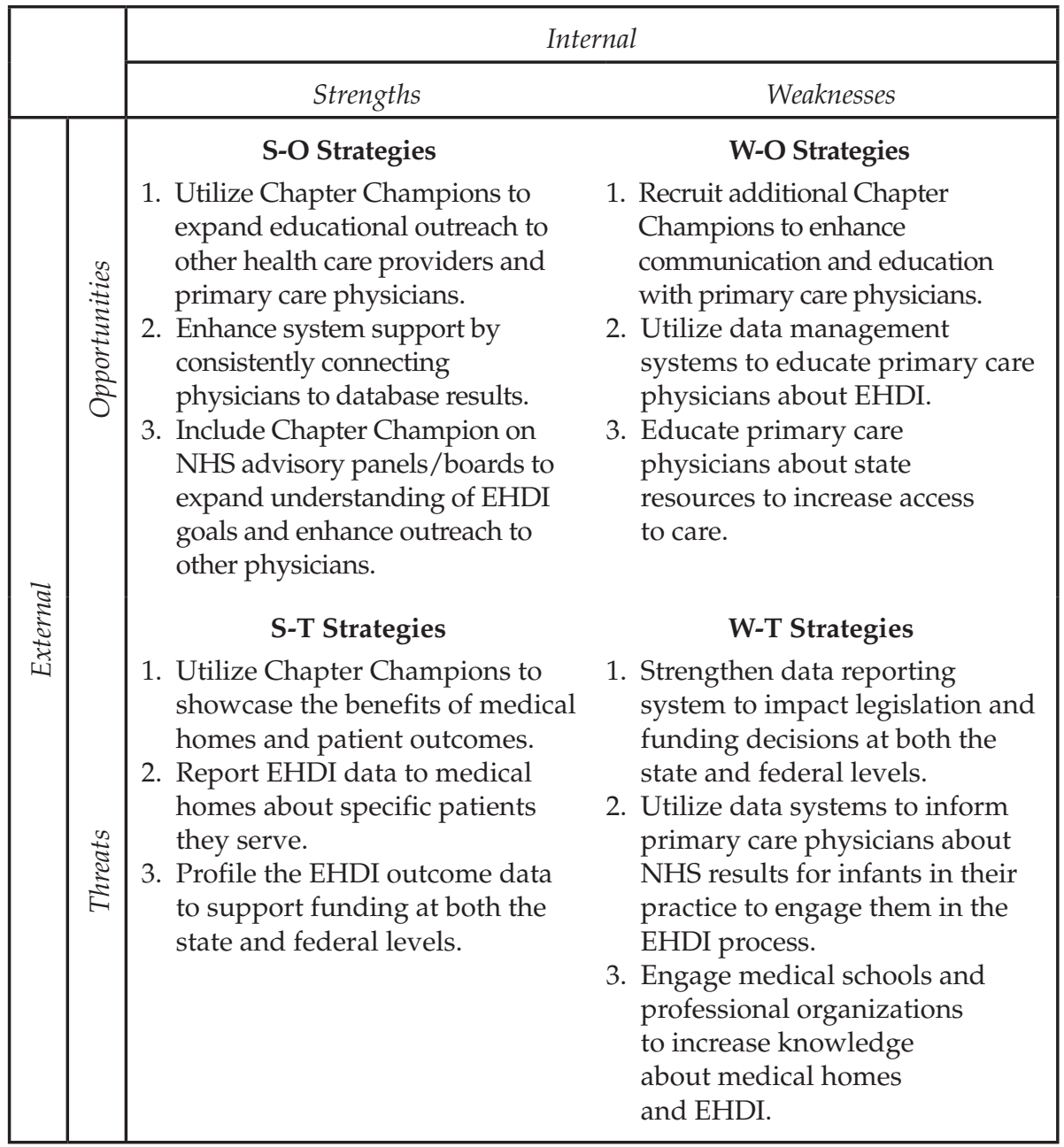

$\mathrm{S}=$ Strength $\mathrm{W}=$ Weakness $\mathrm{O}=$ Opportunity $\mathrm{T}=$ Threat

\section{Limitations}

Limitations of this study include those inherent in the application of SWOT/ TOWS analysis when applied to rapidly changing conditions. SWOT analysis is a subjective tool that explores conditions that are perceived rather than objectively measured. In this sense, the SWOT analysis is influenced by the perspectives of those who completed the surveys as well as those who analyzed the responses. Furthermore, SWOT analysis characterizes the situation at a specific point in time. In addition, SWOT analysis, as applied here, 
resulted in overlapping themes within a category. For example, education was considered a strength when a system was in place that was accessible to physicians and met their learning needs. Education was also considered a weakness when there was no plan in place to address physician educational needs. At the same time it was viewed as an opportunity when the state EHDI coordinator had a plan in place to implement and/or improve the educational process for physicians related to EHDI issues (see White \& Blaiser, 2011, for additional information regarding the selection of SWOT/TOWS for this study and further comment regarding interpretation and application of findings).

\section{Summary}

The responses provided by EHDI coordinators in the SWOT analysis revealed that there is considerable variability among states. The responses, however, did not provide sufficient detail to fully understand how states utilize their strengths to achieve success in integrating the medical home into the EHDI system. Even so, the themes that emerged reveal several factors that are important for state EHDI programs to consider. It is notable that the two most commonly cited weaknesses and opportunities were related to data systems and education. If physicians are to provide an effective medical home for children with hearing loss, they need information that includes test results (screening and assessment) reported in a timely manner; awareness of where to refer children for appropriate services (Harlow \& Bower, 2009); awareness of best practices for children who are suspected of having, are at risk for, or who have been diagnosed with a hearing loss; and access to materials for parents (JCIH, 2007). The AAP has developed several resources for physicians including the "Early Hearing Detection and Intervention (EHDI) Guidelines for Medical Home Providers," which is available online at www.medicalhomeinfo.org/how/clinical_care/hearing_screening. A structured approach to meet physician learning needs is required for systematic communication and dissemination of up-to-date information. States reported that Chapter Champions are the most important element in integrating the medical home into the EHDI process; some noted that having more than one Chapter Champion would be advantageous. The AAP has provided significant resources for pediatricians; however, not all children have a pediatrician as their primary care physician. The same educational outreach is needed for family practice physicians, physician assistants, and other health care providers in the child's medical home.

State EHDI coordinators provided insights into the current issues surrounding medical home integration and what they view as the critical elements. Although an understanding of the specific aspects that make an issue a strength for some states and a weakness for others may serve as a guide for action planning to improve the integration of the medical home into the EHDI 
system, the responses are based on the coordinators' experiences and general impressions. Although studies have explored physician views on the utility of various mechanisms for obtaining information, such as grand rounds (Muñoz, et al., 2009), there is a lack of evidence on what mechanisms actually lead to changes in EHDI-related practice. Future studies are needed to provide information on what strategies lead to improved integration of the medical home in the EHDI process and the context in which they were successful. Examination of the issues in different geographical regions and within a state will be important for understanding which strategies are most effective. Ultimately, the goal is to improve patient care throughout the EHDI process. While we can learn much from the experiences of others, local and regional differences must be considered in the implementation of a statewide plan to optimize the connection of the medical home to the EHDI process.

\section{Acknowledgement}

The work reported in this article was funded in part by the Maternal and Child Health Bureau under Cooperative Agreement \# U52MC04391 with the National Center for Hearing Assessment and Management at Utah State University. The opinions expressed in the article are those of the authors and do not necessarily reflect those of the Bureau.

\section{References}

American Academy of Pediatrics (AAP) Medical Home Initiatives for Children with Special Needs Project Advisory Committee. (2002). The medical home. Pediatrics, 110, 184-186.

Harlor, A. D., \& Bower, C. (2009). Hearing assessment in infants and children: Recommendations beyond neonatal screening. Pediatrics, 124, 1252-1263.

Joint Committee on Infant Hearing (JCIH). (1971). Joint statement on neonatal screening for hearing impairment. Retrieved May 18, 2011, from http:/ / www. jcih.org/history.htm.

Joint Committee on Infant Hearing (JCIH). (2007). Year 2007 position statement: Principles and guidelines for early hearing detection and intervention programs. Pediatrics, 120(4), 898-921.

Kennedy, C. R., McCann, D. C., Campbell, M. J., Law, C. M., Mullee, M., Petrou, S., et al. (2006). Language ability after early detection of permanent childhood hearing impairment. New England Journal of Medicine, 354(20), 2131-2141.

Moeller, M. P. (2000). Early intervention and language development in children who are deaf and hard of hearing. Pediatrics, 106, E43.

Moeller, M. P., Eiten, L. E., White, K. R., \& Shisler, L. (2006). Strategies for educating physicians about newborn hearing screening. Journal of the Academy of Rehabilitative Audiology, 39, 11-32. 
Moeller, M. P., White, K. R., \& Shisler, L. (2006). Primary care physicians' knowledge, attitudes, and practices related to newborn hearing screening. Pediatrics, 118, 1357-1370.

Muñoz, K., \& Nelson, L. (2010, March). Success and challenges to providing timely infant diagnostic hearing assessments. Paper presented at the meeting of Early Hearing Detection and Intervention, Chicago, IL.

Muñoz, K., Shisler, L., Moeller, M. P., \& White, K. (2009). Improving the quality of early hearing detection and intervention services through physician outreach. Seminars in Hearing, 30(3), 184-192.

National Center for Hearing Assessment and Management (NCHAM). (2010). State EHDI/UNHS mandates: Summary table. Retrieved May 17, 2011, from http://www.infanthearing.org/legislative/summary/index.html.

White, K. R. (2007). Early intervention for children with permanent hearing loss: Finishing the EHDI revolution. The Volta Review, 106(3), 237-258.

White, K., \& Blaiser, K. (2011). Strategic planning to improve early hearing detection and intervention programs. The Volta Review, 111(2), 83-108.

White, K., Forsman, I., Eichwald, J., \& Muñoz, K. (2010). The evolution of early hearing detection and intervention programs in the United States. Seminars in Perinatology, 34(2), 171-179.

Yoshinaga-Itano, C., Sedey, A. L., Coulter, D. K., \& Mehl, A. L. (1998). Language of early and later-identified children with hearing loss. Pediatrics, 102(5), 1161-1171. 
The Volta Review, Volume 111(2), Summer 2011, 165-180

\title{
Loss to Follow-Up: Issues and Recommendations
}

\author{
Jeff Hoffman, M.S., CCC-A; Karen F. Muñoz, Ed.D., CCC-A; \\ Tamala S. Bradham, Ph.D., CCC-A; and Lauri Nelson, Ph.D.
}

State coordinators of early hearing detection and intervention (EHDI) programs completed a strengths, weaknesses, opportunities, and threats, or SWOT, analysis that examined 12 areas within state EHDI programs. Related to how EHDI programs address loss to follow-up, 47 coordinators responded with 277 items, and themes were identified in each category. A threats, opportunities, weaknesses, and strengths, or TOWS, analysis yielded 12 recommendations that focused on similar themes. Out of these 12 recommendations, 4 distinct strategies emerged for decreasing loss to follow-up: 1) expand relationships to further develop the EHDI system;2) continue development of comprehensive integrated data systems; 3 ) refine reporting, tracking, and follow-up protocols; and 4) increase the access to services in underserved areas. Addressed appropriately, these recommendations can reduce loss to follow-up in state EHDI programs.

\section{Introduction}

Loss to follow-up (LTF) is a challenging issue facing the early hearing detection and intervention (EHDI) system. Each year, the percentage of infants who receive a hearing screening increases as does the percentage who complete the follow-up process, as measured by the annual, voluntary EHDI Hearing Screening and Follow-up survey conducted by the Centers for Disease Control and Prevention (CDC). However, LTF remains high, with $46.1 \%$ of infants LTF from newborn hearing screening to diagnosis and 33.9\% LTF from diagnosis to early intervention in 2007 (CDC, 2010a). The percentage of LTF

Jeff Hoffman, M.S., CCC-A, is a Senior Research Scientist - Audiologist in the National Center for Hearing Assessment and Management at Utah State University. Karen F. Muñoz, Ed.D., CCC-A, is an Assistant Professor in the Department of Communicative Disorders and Deaf Education at Utah State University. Tamala S. Bradham, Ph.D., CCC-A, is an Assistant Professor in the School of Medicine, Department of Hearing and Speech Sciences, at Vanderbilt University. Lauri Nelson, Ph.D., is an Assistant Professor in the Department of Communicative Disorders and Deaf Education at Utah State University. Correspondence concerning this manuscript should be directed to Mr. Hoffman at jeffhoffman.ehdi@gmail.com. 
from newborn hearing screening to diagnosis varies by state or territory, ranging from $2.0 \%$ to $95.6 \%$ (CDC, 2010a). Increasingly, efforts to reduce the loss to follow-up rate have been the focus of the federal funders of EHDI programs and of various professional organizations. Healthy People 2020, designed to establish benchmarks and monitor progress toward national health objectives, includes an objective related to improving the follow-up rate in the EHDI system nationally:

"Increase the proportion of newborns who are screened for hearing loss by no later than age 1 month, have audiological evaluation by age 3 months, and enroll in appropriate intervention services no later than age 6 months" (U.S. Department of Health and Human Services[DHHS], 2010).

LTF occurs when a baby does not receive the recommended follow-up services. It may occur at any point in the EHDI process, from screening to evaluation or from diagnosis to intervention. There are no standard definitions of LTF across EHDI programs.

Another group with missing data are those classified as "lost to documentation" (Mason, Gaffney, Green, \& Grosse, 2008). Babies in this group are those who have received screening, diagnostic, or intervention services but the results have not been reported to the EHDI program by the service provider. Therefore, the services cannot be documented. In a study of loss to documentation in dried bloodspot screening programs, Hoff, Hoyt, Therrell, and Ayoob (2006) found that name changes, data mismatches, incomplete or erroneous parent contact information, and an unknown primary health care provider contributed to loss to documentation. EHDI programs, however, cannot identify which cases are LTF and which are lost to documentation, since follow-up information is missing for both groups. Therefore, both are typically considered to be LTF.

A further confounding factor in determining LTF rates results from whether the data that is reported to the EHDI program in a state or territory is individually-identifiable or aggregate. Aggregate data often include duplicates where results are reported for the same child by different providers and are, therefore, counted twice. Conversely, aggregate numeric data may appear to indicate a completion of services (one baby diagnosed with hearing loss and enrolled in Part C early intervention) when, in reality, the numbers in an aggregate report may actually be two different babies, one of whom was diagnosed with a hearing loss and did not receive early intervention services (LTF) and the other may not have been reported as diagnosed with a hearing loss (loss to documentation) but did receive early intervention services. In essence, aggregate reporting to EHDI programs may under-report LTF and loss to documentation.

In its annual survey of EHDI programs, the CDC collects aggregate data based on individually-identifiable data at the state level about infants 
with "no documented diagnosis." This includes infants whose parents declined services, are nonresidents, moved out of the state or territory, cannot be contacted, or are unresponsive as well as infants who are in the process of receiving services, who died, or who have no documented diagnosis for reasons that have not been reported to the EHDI program. Infants who comprise the LTF/loss to documentation group are those with "no documented diagnosis" for one of three reasons: 1) parents are unable to be contacted, 2) parents are unresponsive, or 3) unknown reasons (CDC, 2010a).

The CDC, which has provided funding to state EHDI programs for the development of data systems, has identified factors necessary to complete follow-up (CDC, 2003), including:

- Availability of current hearing screening data, diagnostic results, and early intervention documentation for all babies.

- Written policies and procedures for the data tracking system.

- Policies and procedures to ensure privacy and the confidentiality of data, including informed consent requirements.

- Inclusion of all births for the state.

- Identification of risk factors for progressive or later onset hearing loss.

- Report of hearing screening results within one week to ensure timely follow-up.

- A mechanism to report the results of any hearing testing and interventions to all providers.

- Ability to identify all young children at each stage of the EHDI process.

- Access to relevant information about an individual child's hearing status for authorized case managers and health care providers.

In 2008, the American Speech-Language-Hearing Association (ASHA) published a technical report on LTF in the EHDI system based on a systematic review of the evidence by the National Center for Evidence-Based Practice in Communication Disorders (N-CEP), consensus data, and policy statements (ASHA, 2008). Three types of issues were identified as contributing to LTF: systems issues, family issues, and quality assurance issues.

Systems issues contributing to LTF included accurate identification of the baby's primary care physician, communication of hearing screening results to the primary care physician, and the consistency and timing of parent education about newborn hearing screening and explanation of the results and recommended follow-up (ASHA, 2008). Other system issues were the coordination of services, including the lack of integrated data systems and the potential barriers of privacy laws and regulations, i.e., Health Insurance Portability and Accountability Act (HIPAA), Family Education Rights and Privacy Act (FERPA) and Part C Privacy Regulations (Individuals with Disabilities Education Act [IDEA]). Consistent funding for EHDI programs and reimbursement rates for 
pediatric audiological services were also identified as system issues related to LTF.

The second major factor contributing to LTF involved family issues. These included maternal demographics, such as educational and literacy levels, age, marital status, and substance abuse as well as socio economic factors (ASHA, 2008). Finally, the major quality assurance issue to identify and minimize LTF at all stages of the EHDI process can best be addressed with a comprehensive EHDI tracking and surveillance data system (ASHA, 2008).

In 2006, the Health Resources and Services Administration/Maternal and Child Health Bureau (HRSA/MCHB) launched a learning collaborative, National Initiative for Children's Healthcare Quality (NICHQ), to determine effective practices to reduce the LTF rate in the transitions from newborn hearing screening to audiological evaluation and from diagnosis to early intervention (Russ, Hanna, DesGeorges, \& Forsman, 2010). The effective practices identified through a "small test of change" improvement model for the NICHQ were:

- Script the message given the parents when an infant does not pass the hearing screening.

- Obtain a second point of contact for the family, e.g., a relative or friend.

- Verify the identity of the baby's primary care physician before leaving the hospital.

- Make the next appointment for the family before they leave the hospital and explain why it is important to keep the appointment.

- Make reminder calls before appointments and stress the importance of the appointment.

- Make two audiology appointments so that the infant who can't be completely tested at the first appointment is already scheduled to return in a reasonable timeframe.

- "Fax-back" to alert the primary care physician of screening results and the need for prompt follow-up.

- "Fax back" between specialists, including the audiologist and primary care physician.

- Obtain consent for release of information at first contact with early intervention so that information can be entered in the state database.

- Provide clinically useful and timely early intervention reports to primary health care providers.

In 2009, HRSA/MCHB provided supplemental grant funding to EHDI programs to increase the level of effort to reduce LTF based on the effective practices identified during the NICHQ. An analysis of 29 narratives in the supplemental grant applications posted to the NCHAM website (NCHAM, 2010) found that, in addition to the strategies identified in the learning collaborative, EHDI programs were focusing on strategies that are based on the 
needs of their particular EHDI systems (Hoffman, 2010). The primary themes in the grant applications included:

- NICHQ Effective Practices (39 instances), including the practices identified during the learning collaborative as effective in reducing the LTF rate.

- Family Support (32 instances) activities, including surveys and focus groups to identify barriers to follow-up, support for the development of family-to-family support systems, and the creation of resource materials to guide parents through the steps of the follow-up system.

- Data System (22 instances) upgrades and integration with other child health data systems, necessary to better track the services provided.

- Parent Education Materials (22 instances) for parents of newborns and for those whose babies have been identified with a hearing loss need to be developed, revised, and/or translated into the various languages of the populations served.

- Quality Measures (22 instances) for birthing facilities, including report cards based on referral and LTF rates as well as comparative reports.

- Equipment (16 instances) funding through a variety of mechanisms to replace aging equipment in birthing facilities. In addition, expansion of screening to include midwives and public and community health clinics, and improving the diagnostic practices of pediatric audiologists by funding the acquisition of new hearing screening and diagnostic equipment, were also identified as viable approaches to reducing the number of babies who are LTF.

- Education for Audiologists (15 instances), especially those in underserved areas, to provide pediatric diagnostic services as well as educating audiologists about the importance of reporting the results to the EHDI program.

- Education of Hospital Screening Staff (15 instances) to improve both screening practices and reporting hearing screening results to the state EHDI program.

- EHDI Staff (14 instances) being more involved in follow-up with families and professionals to reduce the number of babies who are LTF.

- Improved Reporting to Primary Care Physicians (14 instances) by hospital staff, audiologists, and EHDI program staff to support these providers as key to improving the follow-up outcomes.

- Increased Awareness (13 instances) by professionals and the public, including parents, by improving EHDI websites, developing public service announcements in English and Spanish, and developing press releases.

The purpose of this study was to complete a systematic review using a strengths, weaknesses, opportunities, and threats, or SWOT, and a threats, opportunities, weaknesses, and strengths, or TOWS, analysis to determine 
current EHDI practices regarding LTF issues, and to identify factors to improve program effectiveness and reduce LTF rates.

\section{Methods}

Fifty-one EHDI coordinators were asked to complete an online survey. To examine issues related to LTF, the survey asked for EHDI coordinators to report at least 1 strength, weakness, opportunity, and threat for use in strategic planning. The responses obtained were reviewed by a panel of experts and categorized into common themes within four strategic planning areas. To generate recommendations from the SWOT analysis, a TOWS matrix was used to match identified strengths with opportunities (S-O strategy), strengths with threats (S-T strategy), weaknesses with opportunities (W-O strategy), and weaknesses with threats (W-T strategy). For an in-depth review of the SWOT/ TOWS analysis and its use in the current study, the reader is referred to White and Blaiser, 2011.

\section{Results}

Forty-seven state EHDI coordinators (92\%) completed the LTF area of the SWOT questionnaire. Respondents identified 80 items in the strength category, 65 items in the weakness category, 68 in the opportunity category, and 64 in the threat category for a total of 277 responses. Based on the responses, several specific themes were generated for each category: 7 for strengths, 10 for weaknesses, 9 for opportunities, and 12 for threats (Table 1). Each category also had a miscellaneous section that included no comment, N/A, or items that did not fit within the themes identified.

\section{Strength Characteristics}

The most frequently cited strengths were relationship building (21\%), coordinated tracking protocol (19\%), and program involvement (18\%) (Table 2). Related to relationship building, EHDI coordinators reported they maintained contracts with the primary care physicians, including the American Academy of Pediatrics (AAP) EHDI Chapter Champions, medical homes, hospitals, audiologists, and early interventionists; had strong relationships between EHDI programs in bordering states to ensure that follow-up is completed across state lines; established better relationships with parents through in-service counseling training, including a parent consultant as paid staff to contact families; and established outreach efforts to reduce health disparities for specific communities with poor access to services.

A coordinated tracking protocol was identified as a strength by $19 \%$ of the EHDI coordinators. Specifically, EHDI programs noted effective inpatient and outpatient protocols improved tracking. In addition, an increased number 
Table I. Frequency of themes within each SWOT category for loss to follow-up $(\mathrm{n}=277)$

\begin{tabular}{|c|c|c|}
\hline SWOT category & Themes & Frequency $n(\%)$ \\
\hline Strengths $(n=80)$ & $\begin{array}{l}\text { Relationship Building } \\
\text { Coordinated Tracking Protocol } \\
\text { Program Improvement } \\
\text { Data Tracking System } \\
\text { Access to State Systems } \\
\text { Miscellaneous/No Comment } \\
\text { Funding } \\
\text { State Size }\end{array}$ & $\begin{array}{l}17(21 \%) \\
15(19 \%) \\
14(18 \%) \\
12(15 \%) \\
11(14 \%) \\
4(5 \%) \\
4(5 \%) \\
3(4 \%)\end{array}$ \\
\hline Weaknesses $(n=65)$ & $\begin{array}{l}\text { Protocol } \\
\text { Limited Personnel } \\
\text { Access to Care } \\
\text { Data Management System } \\
\text { Family Circumstances } \\
\text { Lack of Legislation } \\
\text { Interagency/Interdisciplinary Cooperation } \\
\text { Miscellaneous/No Comment } \\
\text { Lack of Quality Improvement System } \\
\text { Lack of Resources }\end{array}$ & $\begin{array}{r}17(26 \%) \\
9(14 \%) \\
8(12 \%) \\
7(11 \%) \\
7(11 \%) \\
6(9 \%) \\
5(8 \%) \\
4(6 \%) \\
1(2 \%) \\
1(2 \%)\end{array}$ \\
\hline Opportunities $(\mathrm{n}=68)$ & $\begin{array}{l}\text { Relationship Building } \\
\text { Data Tracking Systems } \\
\text { Access to State Systems } \\
\text { Quality Improvement } \\
\text { Educational Activities } \\
\text { Coordinated Tracking Protocols } \\
\text { Funding } \\
\text { New Staff } \\
\text { Miscellaneous/No Comment }\end{array}$ & $\begin{array}{c}13(19 \%) \\
10(15 \%) \\
9(13 \%) \\
8(12 \%) \\
7(10 \%) \\
7(10 \%) \\
6(9 \%) \\
4(6 \%) \\
4(6 \%)\end{array}$ \\
\hline Threats $(n=64)$ & $\begin{array}{l}\text { Funding } \\
\text { Interagency Cooperation } \\
\text { Family Circumstances } \\
\text { Limited Personnel } \\
\text { Miscellaneous/No Comment } \\
\text { Location } \\
\text { Linguistic/Cultural Barriers } \\
\text { Change Management } \\
\text { Limited Resources } \\
\text { Time } \\
\text { Educational Needs } \\
\text { Lack of Legislation }\end{array}$ & $\begin{array}{l}15(23 \%) \\
8(13 \%) \\
7(11 \%) \\
7(11 \%) \\
6(9 \%) \\
5(8 \%) \\
4(6 \%) \\
3(5 \%) \\
3(5 \%) \\
3(5 \%) \\
2(3 \%) \\
1(2 \%)\end{array}$ \\
\hline
\end{tabular}

of staff dedicated to tracking was a successful strategy as was having more systematic protocols to classify a case as LTF. EHDI programs also identified "fax back" activities, following the effective practices of the NICHQ Learning Collaborative, as a reason for successful tracking. 
Table 2. Top three themes for strengths

\begin{tabular}{ll}
\hline \multicolumn{1}{c}{ Themes } & \multicolumn{1}{c}{ Descriptions } \\
\hline Relationship Building & Have parent consultants to contact families. \\
& Have strong relationship with border states as \\
& well as minority populations. \\
& Continuous communication with audiologists and \\
& medical homes. \\
Coordinated Tracking Protocol & Partner with tertiary care facilities to provide and \\
& track hearing screenings across the state. \\
& Implemented fax-back forms from screening \\
& facilities to EHDI and medical homes. \\
& Dedicated staff to tracking and following-up with \\
families. & High hospital hearing screening rates. \\
Program Improvement & (i.e., families keeping appointments and not lost \\
& to follow-up). \\
\hline
\end{tabular}

Program improvement was identified as a strength by $18 \%$ of the respondents including the percentage of change over time in improvement in LTF data, although respondents were not specific on what contributed to these changes over time. High inpatient hearing screening rates were also reported in this area.

An effective data tracking system that improves the follow-up rate was a program strength for $15 \%$ of the respondents. A comprehensive data system that tracks screening and diagnostic results, especially if the system is webbased and linked with other data systems, such as the birth certificate system, provided the opportunity to better analyze data. Consistent reporting by providers, even if not accomplished electronically, was also important.

Other strengths included access to state systems (14\%), such as having access to other state registries to find families that were LTF; funding $(5 \%)$, such as access to federal grants; and state size ( $4 \%)$.

\section{Weakness Characteristics}

The three most frequently cited weaknesses were protocols $(26 \%)$, limited personnel $(14 \%)$, and access to care (12\%) (Table 3). Under protocols, EHDI coordinators identified several issues that impacted their LTF rates. Specifically, the transition from screening to diagnostic activities was especially difficult if appointments were not made at the time of referral or if there were delays in making the appointment because of long waitlists or the need to obtain medical insurance authorization. Lack of pertinent information, such as the baby's primary care physician or family contact information, interfered with effective follow-up. Inconsistent reporting of audiological diagnostic evaluation 
Table 3. Top three themes for weaknesses

\begin{tabular}{ll}
\hline \multicolumn{1}{c}{ Themes } & \multicolumn{1}{c}{ Descriptions } \\
\hline Protocol & Length of time to be seen by an audiologist is significantly \\
& delayed (i.e., will not schedule until they get insurance \\
& authorizations or not enough providers). \\
& Poor reporting of data by medical homes/physicians and \\
& audiologists. \\
& Cumbersome protocol to follow. \\
& Lack of providers (e.g., audiologists, physicians). \\
& Overworked EHDI staff. \\
& Limited staff, department reductions, and hiring freezes \\
& result in limited staff for follow-up. \\
Limited Personnel & Inadequate resources for ensuring appropriate follow-up care \\
in low-income, minority, and/or rural communities. & Limited number of diagnostic audiology appointments in the \\
Access to Care & state. \\
\hline
\end{tabular}

results, sometimes due to cumbersome reporting mechanisms, was also identified as a protocol weakness.

Limited personnel, primarily EHDI program staff available to track and follow-up with more difficult cases, was a significant weakness (14\%). This was related to factors such as lack of funding, hiring freezes, and staff reductions. Access to care contributed to LTF as well and was attributed to a lack of providers in some geographical areas and family demographics, including income level, minority status, and place of residence (e.g., rural, densely populated, underserved/unserved areas).

Just as a functional data tracking system was a strength for many EHDI programs, an ineffective data management system was considered a weakness by $11 \%$ of respondents. Coordinators also reported instances where the electronic reporting system was cumbersome, incomplete, or not linked to other child data systems. Another noted contributor to LTF was family circumstances $(11 \%)$. Difficulty in establishing or maintaining contact with families who moved frequently or changed phone numbers was most often reported, and a lack of transportation to travel to follow-up appointments also increased the LTF rate.

Other identified weaknesses included lack of legislation (9\%), lack or limited interagency or interdisciplinary cooperation ( $8 \%$ ), lack of quality improvement system to monitor and improve the EHDI system (2\%), and inadequate resources $(2 \%)$.

\section{Opportunities for EHDI Programs}

The three most frequently cited opportunities were relationship building $(19 \%)$, data tracking systems (15\%), and access to state systems (13\%) (Table 4). 
Table 4. Top three themes for opportunities

\begin{tabular}{|c|c|}
\hline Themes & Descriptions \\
\hline Relationship Building & $\begin{array}{l}\text { Active stakeholders. } \\
\text { EHDI and early intervention are in the same department/ } \\
\text { division/agency. } \\
\text { Increasing communication within community teams } \\
\text { through the replication of the NICHQ Learning } \\
\text { Collaborative project. }\end{array}$ \\
\hline Data Tracking Systems & $\begin{array}{l}\text { Linking data management systems to allow for better } \\
\text { tracking of this population (e.g., vital records, audiologists, } \\
\text { hospitals, early intervention, medical homes). } \\
\text { Using an online system for ease of reporting data. } \\
\text { Creating alternative contact information fields in the data } \\
\text { management system. }\end{array}$ \\
\hline Access to State Systems & $\begin{array}{l}\text { Linkage of data management systems to allow for tracking } \\
\text { and locating families. } \\
\text { Mandatory electronic reporting by all service providers. } \\
\text { Obtaining access to the early intervention and or health } \\
\text { department records. }\end{array}$ \\
\hline
\end{tabular}

EHDI coordinators noted outreach to physicians/medical homes, audiologists, early intervention providers, Early Head Start programs, and state legislators helped build relationships. Respondents also noted that engaging the stakeholders, developing teams to work on key issues in the state, and reaching out to potential sites in the local communities to conduct follow-up hearing screenings helped to build relationships.

In addition to being a strength and a weakness, data tracking systems (15\%) were also identified as an opportunity to reduce LTF. Respondents noted acquiring new web-based data systems, expanding current systems to include more information needed for follow-up, linking with other state data systems, and granting access to additional users (e.g., audiologists, primary care physicians) as opportunities. Access to state systems was reported by $13 \%$ of the EHDI coordinators as an opportunity who identified access, linkage, or integration with other data systems, including medical, public health, and early intervention data systems, as a way to reduce LTF.

Opportunities for quality improvement were identified by $12 \%$ of the EHDI coordinators. Participating in the National Initiative for Children's Healthcare Quality (NICHQ) Learning Collaborative was seen as an approach to program improvement as were plans to implement program evaluations. As data reporting elements change and definitions for LTF are refined, respondents viewed these as opportunities to improve their systems and outcomes.

Ten percent of the respondents perceive educational activities as an opportunity to complete the recommended follow-up services. Program coordinators 
identified the primary recipients of educational efforts as providers (e.g., hospital personnel, audiologists) and primary care physicians. Additionally, the potential to educate the general public through public service announcements and printed materials was also noted. Ten percent of the EHDI coordinators also identified coordinated tracking protocols as an opportunity. Improved reporting practices, such as the "fax back" system, and obtaining parent permission to release information early in the EHDI process were activities with the potential to reduce LTF. Additionally, developing a centralized tracking system was viewed as a mechanism to coordinate the tracking of infants needing follow-up.

Additional opportunities identified included funding (9\%) and new staff (6\%). Specifically, having secured funding for uninsured infants for hearing screenings and evaluations, grant opportunities, and new follow-up coordinator positions within EHDI were all identified as opportunities to help reduce LTF.

\section{Threats to EHDI Programs}

The three most frequently cited threats identified by EHDI coordinators were funding $(23 \%)$, interagency cooperation (13\%), and family circumstances (11\%) (Table 5). Related to funding, 25\% of EHDI coordinators identified a state fiscal crisis and insufficient funds to fully implement a comprehensive EHDI program, including family support and data systems. Follow-up efforts were reduced with fewer staff available. Issues related to insurance coverage also threatened the completion of the recommended follow-up services. Other

Table 5. Top three themes for threats

\begin{tabular}{ll}
\hline \multicolumn{1}{c}{ Themes } & \multicolumn{1}{c}{ Descriptions } \\
\hline Funding & Budget cuts. \\
& Lack of funding. \\
& Lack of or limited insurance coverage. \\
Interagency Cooperation & Confidentiality laws and regulations do not allow for \\
& data sharing. \\
& Not a priority. \\
& Various programs within EHDI do not always work \\
& together. \\
& Transient population. \\
Family Circumstances & Change names, do not return calls, provide false \\
& contact information because they do not want to be \\
& contacted/found. \\
Moving across state lines.
\end{tabular}


items noted in this category included a total reliance on federal funds and the lack of permanent funding.

Twenty-three percent of EHDI coordinators listed factors that reduce interagency cooperation, primarily barriers to sharing follow-up information, as contributing factors to loss to documentation. Information sharing, often guided by federal privacy regulations, was identified as an issue with early intervention and also EHDI programs in other states. Inconsistent and different priorities were identified as contributing to a lack of cooperation between agencies.

Another category to emerge in multiple areas of the SWOT analysis, including threats, was family circumstances. Specifically, $11 \%$ of EHDI coordinators stated that this is a transient population. People move, phone number changes, names change, and some families give false contact information to avoid being identified (e.g., undocumented workers).

Limited personnel was also noted by $11 \%$ of respondents as a threat to LTF, mostly related to shortages of staff in the EHDI programs, a lack of pediatric audiologists, and staff turnover in hospitals. The geographical location of the program was noted by $8 \%$ of EHDI coordinators. Large, sparsely populated states and small, densely populated states were both viewed as challenges to completing the follow-up process as was having a large number of babies with residence in a state or territory different from the place of birth.

Additional threats included linguistic/cultural barriers $(6 \%)$, change of management $(5 \%)$, limited resources $(5 \%)$, time $(5 \%)$, educational needs $(3 \%)$, and lack of legislation (2\%).

\section{TOWS Analysis Matrix}

By applying the TOWS analysis matrix to the themes identified in the SWOT analysis, specific recommendations, or "strategic options," were derived from the internal and external environmental factors identified by the EHDI coordinators. By systematically pairing the four categories of the SWOT analysis, recommendations can be derived to maximize resources and effect positive change. Based on the SWOT themes, 12 individual recommendations were made to reduce LTF (Table 6). To maximize opportunities, identification of strengths can lead to positive change (S-O strategy). For example, linking data management systems to obtain contact information may reduce the LTF rate. By pursuing opportunities, weaknesses can be minimized (W-O strategy). For example, by engaging the active stakeholders and EHDI providers, current practices can be evaluated and action plans can be developed to make the system more efficient and effective. Identification of ways to use strengths can ultimately reduce vulnerability to threats (S-T strategy). For example, building support with both parents and professionals will result in increased advocacy efforts. Finally, establishing defensive plans will help to prevent weaknesses from making programs susceptible to threats (W-T strategy). For example, streamlining the tracking and follow-up processes will reduce inefficiencies 
Table 6. TOWS analysis matrix for loss to follow-up

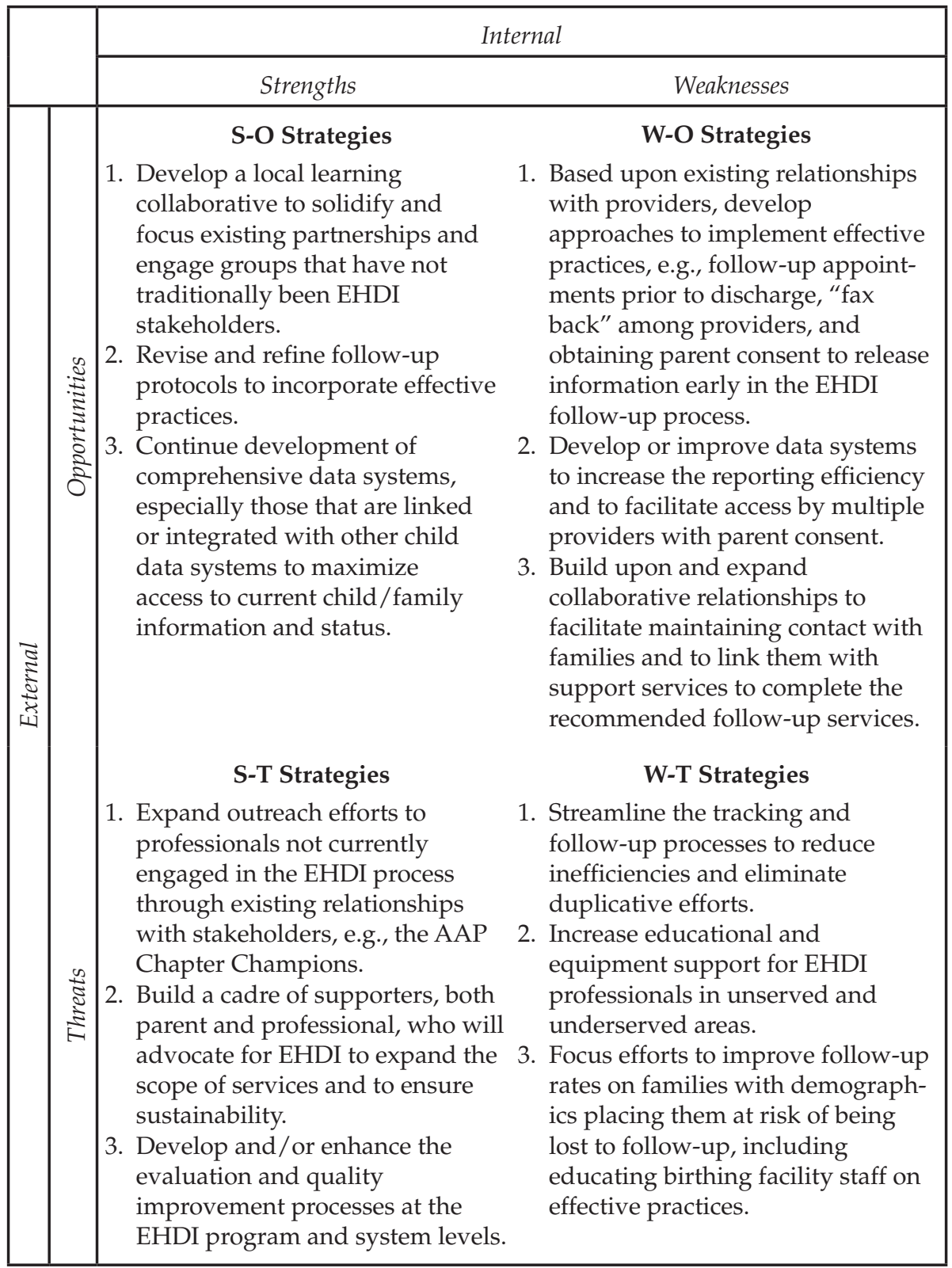

$\mathrm{S}=$ Strength $\mathrm{W}=$ Weakness $\mathrm{O}=$ Opportunity $\mathrm{T}=$ Threat 
and eliminate duplicative efforts. Out of these 12 recommendations, 4 distinct strategies for decreasing LTF emerged. Specifically, state EHDI programs should: 1) expand relationships to further develop the EHDI system by reviewing and updating existing protocols and guidelines to include who is responsible, timelines, and dissemination of reports on how well the program is doing; 2) develop or improve the existing data management system to capture the data with this population; 3) refine reporting, tracking, and follow-up protocols; and 4) focus specifically placed on how to increase access for hearing services in rural areas, minorities, and underserved/unserved populations.

\section{Limitations}

Limitations of this study include those inherent in the application of SWOT / TOWS analysis when applied to rapidly changing conditions. SWOT analysis is a subjective tool that explores conditions that are perceived rather than objectively measured. In this sense, the SWOT analysis is influenced by the perspectives of those who completed the surveys as well as those who analyzed the responses. Furthermore, SWOT analysis characterizes the situation at a specific point in time. In addition, the SWOT analysis, as applied here, resulted in overlapping themes within a category. For example, funding and data management were identified in three of the four SWOT categories (see White \& Blaiser, 2011, for additional information regarding the selection of SWOT/TOWS for this study and further comment regarding interpretation and application of findings).

\section{Summary}

LTF between screening and diagnosis and between diagnosis and intervention is a major concern for EHDI programs and stakeholders. Respondents noted that establishing and maintaining relationships with stakeholders and parents was considered an effective approach to developing strategies that increased the number of infants receiving the recommended follow-up services, and for the results of those services to be reported to the EHDI program. Similarly, effective EHDI data systems, especially those linked to other child data systems, were both a strength and an opportunity. Respondents were beginning to report improvements in the LTF rate, with mention of implementing some of the effective strategies from the NICHQ Learning Collaborative as a factor. Tracking protocols were both a strength and a weakness, depending on the degree to which the programs were receiving complete and accurate information and results.

Themes that emerged in the SWOT analysis are also reflected in the Reducing Loss to Follow-up HRSA/MCHB supplemental grant applications written several months after the EHDI coordinators completed the SWOT analysis. Although clustered in different ways, four of the top five themes 
for both the grant applications and the SWOT analysis were 1) the application of effective practices from the NICHQ Learning Collaborative; 2) establishing relationships, especially with families; 3) improving data systems to include linkage with other systems; and 4) developing quality improvement mechanisms.

In summarizing the SWOT and TOWS analysis, the following recommendations emerge:

- Existing positive relationships with parents and professionals can be used to engage additional stakeholders in reaching out to other groups and individuals, adopting effective practices, linking families to the recommended services, and enlisting advocates.

- Continual development of comprehensive data systems that are linked to other child data systems, are easy to use and accessible by providers, efficiently support follow-up efforts, and provide for program and system evaluation.

- Reporting, tracking, and follow-up protocols must be refined to incorporate effective practices, to focus on families most at risk of being categorized as LTF, and to maximize the efficiency of conducting follow-up to ensure that the recommended services are received and reported.

- Health disparities must be reduced, especially for high-risk groups and those in unserved or underserved areas.

Each year the percentage of LTF has decreased (CDC, 2010b). With a better understanding of the factors that contribute to or are associated with LTF, the focus on developing approaches to improving follow-up, and the allocation of additional funding, it is hoped that future SWOT and TOWS analysis will reveal a significant reduction in the number of children lost to follow-up.

\section{Acknowledgement}

The work reported in this article was funded in part by the Maternal and Child Health Bureau under Cooperative Agreement \# U52MC04391 with the National Center for Hearing Assessment and Management at Utah State University. The opinions expressed in the article are those of the authors and do not necessarily reflect those of the Bureau.

\section{References}

American Speech-Language-Hearing Association (ASHA). (2008). Loss to follow-up in early hearing detection and intervention [technical report]. Retrieved May 20, 2011, from http://www.asha.org/policy. 
Centers for Disease Control and Prevention (CDC). (2003). Early hearing detection and intervention program guidance manual. Atlanta, GA: Author.

Centers for Disease Control and Prevention (CDC). (2010a). EHDI hearing screening and follow-up survey. Retrieved May 20, 2011, from http://www. cdc.gov/ncbddd/EHDI/data.html.

Centers for Disease Control and Prevention (CDC). (2010b). Identifying infants with hearing loss - United States, 1999-2007. Morbidity and Mortality Weekly Report, 59(8), 220-223

Hoff, T., Hoyt, A., Therrell, B., \& Ayoob, M. (2006). Exploring barriers to longterm follow-up in newborn screening programs. Genetics in medicine, 8 , 563-570.

Hoffman, J. (2010). Managing loss to follow-up in early hearing detection and intervention systems. ASHA Audiology 2010 Online Conference: Early hearing detection and intervention (EHDI). Retrieved October 13, 2010, from http:/ / www. asha.org/eweb/OLSDynamicPage.aspx?webcode=olsmainpage.

Mason, C., Gaffney, M., Green, D. R., \& Grosse, S. D. (2008). Measures of follow-up in early hearing detection and intervention programs: A need for standardization. American Journal of Audiology, 17, 60-67.

National Center for Hearing Assessment and Management (NCHAM). (2010). State coordinator toolbox. Retrieved May 20, 2011, from http://www.infan thearing.org/stategrants/index.html.

Russ, S., Hanna, D., DesGeorges, J., \& Forsman, I. (2010). Improving follow-up to newborn hearing screening: A learning-collaborative experience. Pediatrics, 126, S59-S69.

U.S. Department of Health and Human Services (DHHS). (2010). Healthy people 2020 objectives for improving health. Retrieved May 20, 2011, from http://www.healthypeople.gov/2020/topicsobjectives2020/objectiveslist. aspx?topicid=20.

White, K., \& Blaiser, K. (2011). Strategic planning to improve early hearing detection and intervention programs. The Volta Review, 111(2), 83-108. 
The Volta Review, Volume 111(2), Summer 2011, 181-194

\title{
Strategic Analysis of Family Support in EHDI Systems
}

\author{
Tamala S. Bradham, Ph.D., CCC-A; \\ K. Todd Houston, Ph.D., CCC-SLP, LSLS Cert. AVT; \\ Gayla Hutsell Guignard, M.A., CCC-A/SLP, LSLS Cert. AVT; and \\ Jeff Hoffman, M.S., CCC-A
}

State coordinators of early hearing detection and intervention (EHDI) programs completed a strengths, weaknesses, opportunities, and threats, or SWOT, analysis that examined 12 areas within state EHDI programs. For the family support area, 47 EHDI coordinators listed 255 items, and themes were identified within each category. A threats, opportunities, weaknesses, and strengths, or TOWS, analysis yielded 12 recommendations that focused on similar themes. Out of these 12 recommendations, 3 distinct strategies emerged to improve family support: 1) develop and implement family support programs; 2) engage paid staff members who are parents of children who are deaf or hard of hearing; and 3) utilize appropriate materials created by national, state, and local agencies. Addressed appropriately, these recommendations can strengthen family support services in state EHDI programs.

\section{Introduction}

Each day approximately 33 families in the United States learn that their newborn infant has a permanent hearing loss (White, 1997). The language abilities of children who are identified as deaf or hard of hearing will be affected by the parents' reactions, acceptance, and advocacy for their child (Moeller, 2000).

Tamala S. Bradham, Ph.D., CCC-A, is an Assistant Professor in the School of Medicine, Department of Hearing and Speech Sciences, at Vanderbilt University. K. Todd Houston, Ph.D., CCC-SLP, LSLS Cert. AVT, was a an Assistant Professor in the Department of Communicative Disorders and Deaf Education at Utah State University at the time of this study and is currently at The University of Akron. Gayla Hutsell Guignard, M.A., CCC-A/SLP, LSLS Cert. AVT is the Program Director of Indiana's Early Hearing Detection \& Intervention (EHDI) program in the Department of Genomics \& Newborn Screening at the Indiana State Department of Health. Jeff Hoffman, M.S., CCC-A, is a Senior Research Scientist - Audiologist in the National Center for Hearing Assessment and Management at Utah State University. Correspondence concerning this manuscript should be directed to Dr. Bradham at tamala.bradham@vanderbilt.edu. 
Evidence supports that effective early hearing detection and intervention programs (EHDI) lead to positive outcomes for children who are deaf or hard of hearing in their language and social development (Nelson, Bougatsos, \& Nygren, 2008; Yoshinaga-Itano, 2003, 2004; Yoshinaga-Itano, Sedey, Coulter, \& Mehl, 1998). One component of an effective EHDI program includes the delivery of timely and consistent family support.

According to the National Center for Hearing Assessment and Management (NCHAM), family support is meant to "aid the cause and interests of families who have a deaf or hard of hearing child, and to promote polices that further these causes and interests in a manner which is sensitive to families from racially, ethnically, culturally, and linguistically diverse backgrounds" (NCHAM, 2011). Parents who are emotionally available and connected to their child help support the child's overall development (Kurtzer-White \& Luterman, 2003). Thus, effective family support is essential to an effective EHDI program. The Joint Committee on Infant Hearing (JCIH, 2007) provides several recommendations for family support:

- Parent representatives should be included in advisory board activities.

- Parent(s) can be paid staff members to provide intervention and program support.

- Parent(s) can be involved with training of professionals.

- Parent(s) and family members can assume leadership roles in:

- policy development

- resource material

- communication mechanisms

- mentoring and advocacy opportunities

- dissemination of information

- interaction with the Deaf community and other individuals who are hard of hearing

- Information presented to families should be culturally sensitive and understandable.

- Families should be made aware of all communication options and available hearing technologies.

- Services should be family-centered with infant and family rights and privacy guaranteed.

- EHDI services should include supportive family education and counseling.

\section{Parental Reactions and Stresses of Hearing Loss Diagnosis}

While there is evidence to support the effectiveness of early identification and intervention on outcomes for language and social emotional development, there is little research that defines the impact of the child's hearing loss on the family as a unit. Some studies have examined the processes of 
family adjustment, coping strategies, and stress management of stress (KurtzerWhite \& Lutterman, 2003; Young \& Tattersall, 2007), but research remains limited. Yoshinaga-Itano and deUzcategui (2001) surveyed parental reactions shortly after their newborns did not pass a hearing screening test. Parents in this study reported feelings of fear $(52 \%)$, shock $(42 \%)$, confusion $(42 \%)$, depression $(37 \%)$, frustration $(31 \%)$, anger $(22 \%)$, sadness $(16 \%)$, loneliness $(16 \%)$, and blame (16\%). Young and Tattersall (2007) interviewed 45 parents/ caregivers/extended family members (of 27 families) approximately 25 weeks after their child was diagnosed with a hearing loss (range 8 to 51 weeks old). The majority of the families interviewed "were unequivocally positive about the fact that their child's hearing loss had been identified early." None of the families thought that knowing early alleviated any of the shock, grief, or loss associated with having a child who was deaf or hard of hearing, reporting that these feelings would have happened anyway. Only two mothers reported that they wished they had not known early so they could have enjoyed their newborn baby. Vohr, Letourneau, and McDermott (2001) surveyed 307 mothers at the time of the hearing screen and 40 mothers at the time of the rescreen. The degree of maternal worry was significantly greater at the rescreen compared to the screen, with the mothers of greater socioeconomically disadvantage reporting the highest worry levels. Even with additional knowledge about the hearing screening, the degree of worry remained the same.

In evaluating stress levels of mothers who have children with and without hearing loss, Pipp-Siegel, Sedey, and Yoshinaga-Itano (2002) reported mothers of children with hearing loss do not exhibit more stress than mothers of children with typical hearing. When evaluating different types of parental stresses, Pipp-Siegal, et al., found that up to $16 \%$ of the mothers may experience stress levels that would be considered clinically significant. They also reported that lower income, low levels of perceived support from others, the perception that the daily hassles associated with parenting are especially frequent or troublesome, the presence of disabilities in addition to hearing loss, children whose language lags far behind their chronological age, and children who have less severe degrees of hearing loss are often associated with higher stress levels (Pipp-Siegel, et al., 2002). Vohr and colleagues (2008) administered the Parenting Stress Index to 33 mothers of infants with confirmed hearing loss, 42 of infants with false positive screening results, and 70 of infants in a control group. Mothers of infants in the false positive group did not report increased stress. Factors that contributed to overall stress across all three groups included length of time in the neonatal intensive care unit and the presence of hearing loss. Mothers of older maternal age and greater family resources were associated with decreased stress on the family.

Quality of life is another area that has been examined following early identification of hearing loss. Jackson, Wegner, and Turnbull (2010) administered 
a questionnaire to families of 207 children who were deaf and younger than 6 years of age. Results from descriptive statistics revealed that families were generally satisfied in areas of family life, but reported lower satisfaction ratings in the area of emotional well-being. Family members of a child who used a cochlear implant reported higher satisfaction than family members of children using hearing aids alone.

\section{Impact Factors on Child Development}

There are many variables that affect the overall language and socialemotional development of a child who is deaf or hard of hearing, such as maximizing residual hearing through advanced technologies and audiological services and accessing highly-qualified service providers who can facilitate language development. Other variables that contribute to communication and language development include resolution of grief, maternal-childinteraction, bonding, parental stress, parental emotional availability, and the child's self-development (Kurtzer-White \& Luterman, 2003).

The Kubler-Ross grief model, based on stages of grief people typically experience with loss or change, is often associated with parental grief over their child's hearing loss. These stages include denial, anger, bargaining, depression, and acceptance. While there may be similarities, grief - when associated with death - is often terminable whereas parental grief related to a perceived disability is often cyclical and chronic (Kurtzer-White \& Lutterman, 2003). Recognizing these differences is essential when working with families in EHDI programs.

After the diagnosis of hearing loss, parents are asked to make many decisions that will affect their child's development and their overall family structure. Parental coping behaviors and management of stress will ultimately play a significant role in how they make those decisions. For some parents, their bond may be affected as a result of learning that their child has a special need while others will immediately want to know what they can do to "fix it." How professionals support families and connect them with other support networks will play a significant role in helping the family support their child.

\section{Reaching Families}

The Centers for Disease Control and Prevention (CDC) data indicated that 97\% of newborns in the United States were screened for hearing loss (CDC, 2010). Unfortunately, $46.1 \%$ of infants referred for audiological evaluation after failing the newborn hearing screening were considered to be lost to follow-up/lost to documentation (CDC, 2010). Furthermore, only 53.3\% of the infants diagnosed with hearing loss were enrolled in early intervention services (CDC, 2010). These findings suggest that an absence of adequate support mechanisms exist for families whose child needs additional testing, 
and for most families whose child has been diagnosed with hearing loss and needs early intervention services.

Families find support in many ways (Clark \& Pittman, 2006). First, through both formal and informal parent support programs, sometimes referred to as parent-to-parent (P2P) support programs, where families can connect with others in similar situations. Second, by connecting with an adult who is deaf or hard of hearing to serve as a role model, mentor, or as someone to call on from time to time, families may learn more about the experience of being deaf or hard of hearing, recognize the diverse backgrounds and stories of individuals who have grown up with hearing loss, and receive a glimpse into their own child's future. Third, with the advances in hearing technology and much earlier identification of hearing loss in children, families of newly identified children may also find it beneficial to meet older children and teens whose life experiences more closely reflect those of their own child. Another source of family support is gathering information through the Internet, books, brochures, videos/DVD/CDs, social media, brochures, and other printed materials to help navigate systems and services as well as provide information about hearing loss in children, the unique challenges and joys of parenting a child with hearing loss, and plans for the future. Additionally, professionals, such as audiologists, speech-language pathologists, educators of the deaf involved in early intervention, physicians, surgeons, social workers, and advocates, have a wealth of knowledge that can be shared with families during their continuum of care. Lastly, families also find support in their communities. Whether they find this support through neighbors, friends, or place of worship, reaching out to and accepting support from others can have a powerful impact in helping families during this time of need.

The purpose of this study was to complete a systematic review using a strengths, weaknesses, opportunities, and threats, or SWOT, and a threats, opportunities, weaknesses, and strengths, or TOWS, analysis to determine current EHDI practices in family support, and to identify factors that could improve program effectiveness and lead to better outcomes for infants who are deaf and hard of hearing.

\section{Methods}

Fifty-one EHDI coordinators were asked to complete an online survey. To examine issues related to family support services, the survey asked for the EHDI coordinator to report at least 1 item in the following four areas to assist with strategic planning: strength, weakness, opportunity, and threat. The responses obtained were reviewed by a panel of experts in the field and categorized into common themes in each of these four strategic planning areas. To generate recommendations from the SWOT analysis, a TOWS matrix was used to match identified strengths with opportunities (S-O strategy), strengths with threats (S-T strategy), weaknesses with opportunities (W-O strategy), and 
weaknesses with threats (W-T strategy). For an in-depth review of the methodology, the reader is referred to White and Blaiser, 2011.

\section{Results}

Forty-seven EHDI coordinators (92\%) completed the family support area of the SWOT questionnaire. Respondents identified 82 items in the strength category, 56 items in the weaknesses category, 62 items in the opportunities category, and 55 items in the threats category for a total of 255 responses. Based on the responses, several specific themes were generated for each category: 8 for strengths, 9 for weaknesses, 7 for opportunities, and 5 for threats (Table 1).

Table I. Frequency of themes within each SWOT category $(n=255)$

\begin{tabular}{|c|c|c|}
\hline SWOT category & Themes & Frequency $n(\%)$ \\
\hline Strengths $(\mathrm{n}=82)$ & $\begin{array}{l}\text { Affiliations with National Family Support } \\
\text { Organizations } \\
\text { State/Local Family Support services } \\
\text { Staff Position(s) for Parent Services } \\
\text { Parent Training/Educational Materials } \\
\text { Relationships } \\
\text { Multicultural Services } \\
\text { Financial Support } \\
\text { Advisory Committees/Boards }\end{array}$ & $\begin{array}{l}13(16 \%) \\
13(16 \%) \\
11(13 \%) \\
9(11 \%) \\
6(7 \%) \\
5(6 \%) \\
3(4 \%)\end{array}$ \\
\hline Weaknesses $(n=56)$ & $\begin{array}{l}\text { Program Development Barriers } \\
\text { Inadequate Family Support Services } \\
\text { Geographical Challenges } \\
\text { Communication } \\
\text { Referrals } \\
\text { Parent/Family Participation Barriers } \\
\text { Personnel Shortages } \\
\text { Miscellaneous/No Comments } \\
\text { Funding }\end{array}$ & $\begin{array}{l}15(27 \%) \\
11(20 \%) \\
9(16 \%) \\
5(9 \%) \\
4(7 \%) \\
4(7 \%) \\
3(5 \%) \\
3(5 \%) \\
2(4 \%)\end{array}$ \\
\hline Opportunities (n=62) & $\begin{array}{l}\text { Communication Resources/Materials } \\
\text { Involvement in National Organizations } \\
\text { Collaboration with State \& Local Organizations } \\
\text { Miscellaneous / No Comments } \\
\text { Linking Families to Other Families for Support } \\
\text { Parent Involvement } \\
\text { Funding }\end{array}$ & $\begin{aligned} & 13(21 \%) \\
& 12(19 \%) \\
& 11(18 \%) \\
& 8(11 \%) \\
& 7(11 \%) \\
& 6(10 \%) \\
& 5(8 \%)\end{aligned}$ \\
\hline Threats $(n=55)$ & $\begin{array}{l}\text { Funding } \\
\text { Program Development Barriers } \\
\text { Parental Involvement Barriers } \\
\text { Miscellaneous/No Comments } \\
\text { Geographical Barriers }\end{array}$ & $\begin{aligned} & 21(38 \%) \\
& 12(22 \%) \\
& 9(16 \%) \\
& 8(15 \%) \\
& 5(9 \%)\end{aligned}$ \\
\hline
\end{tabular}


Each category also had a miscellaneous section that included no comment, $\mathrm{N} / \mathrm{A}$, or items that did not fit within the themes identified.

\section{Strength Characteristics}

The three most frequently cited strengths were affiliation with national P2P support organizations ( $27 \%)$, state/local P2P support services $(16 \%)$, and paid staff position(s) for family support services (16\%) (Table 2). Twenty-seven percent of respondents reported that affiliations with national organizations, such as Hands \& Voices and the Alexander Graham Bell Association for the Deaf and Hard of Hearing (AG Bell), aided family support services. Specifically, state EHDI coordinators from states that incorporated the Hands \& Voices' Guide by Your Side (GBYS) program indicated that this involvement had resulted in significant, positive changes (Hands \& Voices, 2011).

Access to state agencies and organizations that provide family support was also an important adjunct to family support programming for $16 \%$ of respondents. EHDI coordinators relied on agencies such as Family Voices, BEGINNINGS for Parents of Children who are Deaf or Hard of Hearing, Family Resource Centers, and/or state early intervention programs to provide family support services in their states. In addition, $16 \%$ of respondents noted that hiring parents of children who are deaf or hard of hearing as EHDI staff helped families better navigating various state and local systems, followup on care, and receive appropriate support materials.

Hosting family support conferences and providing parent information kits and other resources (13\%), building relationships among parents and

Table 2. Top three themes for strengths

\begin{tabular}{|c|c|}
\hline Themes & Descriptions \\
\hline $\begin{array}{l}\text { Affiliations with National } \\
\text { Family Support } \\
\text { Organizations }\end{array}$ & $\begin{array}{l}\text { Active state chapters of national organizations } \\
\text { (i.e., Hands \& Voices, AG Bell, Family Voices, etc.). } \\
\text { Implementing "Guide By Your Side" program. } \\
\text { Paid staff members working with state chapters to } \\
\text { facilitate family support. }\end{array}$ \\
\hline $\begin{array}{l}\text { State/Local Family } \\
\text { Support Services }\end{array}$ & $\begin{array}{l}\text { Establishment of support groups across the state through } \\
\text { both private and public sectors. } \\
\text { Establishment of listservs and web-based materials. } \\
\text { Highly qualified staff to provide the family support } \\
\text { services. }\end{array}$ \\
\hline $\begin{array}{l}\text { Staff Positions for Parent } \\
\text { Services }\end{array}$ & $\begin{array}{l}\text { Dedicated paid parent position(s) to assist families in the } \\
\text { hospital. } \\
\text { Dedicated paid parent position(s) to help families } \\
\text { navigate state systems. } \\
\text { Dedicated paid parent position(s) for family support. }\end{array}$ \\
\hline
\end{tabular}


providers $(11 \%)$, being culturally sensitive $(7 \%)$, and having financial support $(6 \%)$ were also reported to be strengths. Respondents also noted parent involvement on advisory boards (4\%) helped establish positive relationships and build better EHDI programs.

\section{Weakness Characteristics}

The three most frequently cited weaknesses reported by state EHDI coordinators were barriers with program development $(27 \%)$, inadequate family support $(20 \%)$, and significant geographical challenges $(20 \%)$ (Table 3$)$. The elements that contributed to these areas of weakness included lack of coordinated services, lack of leadership or inadequate training of new leaders, and overall lack of services. There were several respondents that reported their states did not have a family support network, state chapters of national family support organizations, or services for families with limited or no English proficiency.

Another reported weakness was limited educational materials that are culturally and linguistically appropriate $(9 \%)$. Coordinators that reported having family support programs cited difficulties in obtaining referrals for those services $(7 \%)$, specifically that referral sources (e.g., medical homes, audiologists, early intervention services) did not know about the programs, did not know how to make the referrals, or made referrals "too late." Parent/family participation barriers $(7 \%)$, personnel shortages $(5 \%)$, and funding $(4 \%)$ were also listed as weaknesses.

Table 3. Top three themes for weaknesses

\begin{tabular}{|c|c|}
\hline Themes & Descriptions \\
\hline $\begin{array}{l}\text { Program Development } \\
\text { Barriers }\end{array}$ & $\begin{array}{l}\text { Time constraints to move this initiative forward to start } \\
\text { family support groups; staffing limitations. } \\
\text { Lack of services, no hearing aid loaner program, no cochlear } \\
\text { implant center. } \\
\text { Part C program lacks experts in hearing loss. }\end{array}$ \\
\hline $\begin{array}{l}\text { Inadequate Family } \\
\text { Support }\end{array}$ & $\begin{array}{l}\text { Limited family support groups. } \\
\text { No Family Resource Center or the Family Resource Center } \\
\text { does not address the needs of families with children who } \\
\text { are deaf or heard of hearing. } \\
\text { Limited support and resources for families with limited } \\
\text { English proficiency. }\end{array}$ \\
\hline $\begin{array}{r}\text { Geographical } \\
\text { Challenges }\end{array}$ & $\begin{array}{l}\text { Support services vary greatly between rural and urban areas. } \\
\text { The geographic nature of the state makes it difficult to } \\
\text { provide culturally competent direct family-to-family } \\
\text { support services available for non-English speakers. } \\
\text { No outreach to rural or frontier areas. }\end{array}$ \\
\hline
\end{tabular}


The three most frequently cited opportunities were informational resources and materials $(21 \%)$, presence of a national organization $(19 \%)$, and collaboration with state and local organizations (18\%) (Table 4). With electronic media and the Internet, respondents reported multiple ways of communicating with families and organizations. Twenty-one percent of EHDI coordinators reported that e-mail, websites, web-cams, social media, and listservs were available for use. Creating brochures, translating current literature into multiple languages, and revising the current literature to include more information were also reported as effective communication strategies.

Nineteen percent of EHDI coordinators reported that state chapters of national organizations helped strengthen family support services. Respondents $(18 \%)$ also noted that collaborations with Early Head Start programs, family resource centers, and nonprofit organizations that provide family support aid the EHDI program's efforts.

Opportunities to link families with other families (11\%), start parent groups and / or organize and host family support conferences (10\%), and funding $(8 \%)$ were also listed. Establishing a state Hands \& Voices chapter and implementing GBYS were also strategies listed by EHDI coordinators. Of interest, $11 \%$ of EHDI coordinators reported no comments or "I don't know" in the opportunities section.

Table 4. Top three themes for opportunities

\begin{tabular}{|c|c|}
\hline Themes & Descriptions \\
\hline $\begin{array}{l}\text { Communication Resources } \\
\text { and Materials }\end{array}$ & $\begin{array}{l}\text { Electronic communications, email, listserv, } \\
\text { webcams, social media. } \\
\text { Development of brochures and educational } \\
\text { materials. } \\
\text { Connecting families to community resources. }\end{array}$ \\
\hline $\begin{array}{l}\text { Involvement with } \\
\text { National Organizations }\end{array}$ & $\begin{array}{l}\text { Establish state chapters of national } \\
\text { organizations (e.g., AG Bell, Hands \& } \\
\text { Voices, etc.). } \\
\text { Implementing Guide By Your Side (GBYS) } \\
\text { program in the state. }\end{array}$ \\
\hline $\begin{array}{l}\text { Collaboration with State } \\
\text { and Local Organizations }\end{array}$ & $\begin{array}{l}\text { Family Support Advisory Sub-Committee } \\
\text { within EHDI. } \\
\text { EHDI collaborating with Early Head Start } \\
\text { programs, family resource centers, educational } \\
\text { programs for children who are deaf or hard of } \\
\text { hearing, and/or other nonprofit organizations } \\
\text { that support families. }\end{array}$ \\
\hline
\end{tabular}


Table 5. Top three themes for threats

\begin{tabular}{ll}
\hline \multicolumn{1}{c}{ Themes } & \multicolumn{1}{c}{ Descriptions } \\
\hline Funding & $\begin{array}{l}\text { No source of funding support for this initiative. } \\
\text { Limited funding. } \\
\text { Lack of sustainable funding and budget cuts. }\end{array}$ \\
$\begin{array}{c}\text { Program Development } \\
\text { Barriers }\end{array}$ & $\begin{array}{l}\text { Disagreements by nonprofit and special-interest groups } \\
\text { create delays and stop progress. } \\
\text { Lack of referrals for family support. } \\
\text { Competing priorities, lack of staff and resources to focus } \\
\text { on this area } \\
\text { Parent Involvement } \\
\text { Barriers }\end{array}$ \\
& $\begin{array}{l}\text { Difficulties reaching parents and families. } \\
\text { Family acceptance of hearing loss and delay in acting upon } \\
\text { the recommendations. }\end{array}$ \\
\hline
\end{tabular}

\section{Threats to EHDI Programs}

The three most frequently cited threats were funding (38\%), barriers to program development $(22 \%)$, and parental involvement (16\%) (Table 5). Overwhelmingly, $38 \%$ of respondents reported that funding, budget cuts, and fiscal crises were all threats to their programs. Several coordinators expressed concern that family support would be the first item targeted for cuts due to the fiscal crises faced by the U.S. government and state governments. Barriers for EHDI program development (22\%) included the impact of stakeholders' disagreements about service delivery models and legislative efforts, the lack of a unified approach on managing children who are deaf or hard of hearing, limited resources and time, lack of appropriate staff members, and limited identification of support needed to meet families' unique needs.

EHDI coordinators (16\%) also expressed concerns about limited family involvement due to the times that meetings are scheduled and lack of parent leadership. Respondents also noted geographical considerations ( $9 \%)$, such as the specific needs of families in rural areas, as a threat.

\section{TOWS Analysis Matrix}

By applying the TOWS analysis matrix to the themes identified in the SWOT analysis, specific recommendations, or "strategic options," were derived from the internal and external environmental factors identified by the EHDI coordinators. By systematically pairing the four categories of the SWOT analysis, recommendations can be derived to maximize resources and effect positive change. Based on the SWOT themes, 12 individual recommendations were made (Table 6). To maximize opportunities, strengths can be used to maximize efficiency and lead to positive change (S-O strategy). For example, 
Table 6. TOWS analysis matrix for family support

\begin{tabular}{|c|c|c|c|}
\hline & \multicolumn{2}{|c|}{ Internal } \\
\hline & & Strengths & Weaknesses \\
\hline \multirow{4}{*}{ 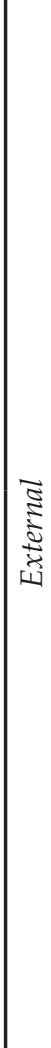 } & & S-O Strategies & W-O Strategies \\
\hline & 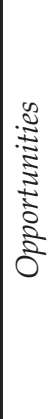 & $\begin{array}{l}\text { 1. Continue to develop and/or } \\
\text { grow state chapters to support } \\
\text { families. } \\
\text { 2. Utilize national resources for } \\
\text { development and dissemination } \\
\text { of materials and resources. } \\
\text { 3. Pay parents to implement } \\
\text { family support programs. }\end{array}$ & $\begin{array}{l}\text { 1. Utilize "outside" family support } \\
\text { programs to provide family } \\
\text { support. } \\
\text { 2. Provide resources about EHDI to } \\
\text { Family Resource Centers. } \\
\text { 3. Connect families in rural/ } \\
\text { frontier areas with other families } \\
\text { via traditional and electronic } \\
\text { communications and to local } \\
\text { collaborating agencies and } \\
\text { organizations. }\end{array}$ \\
\hline & & S-T Strategies & W-T Strategies \\
\hline & 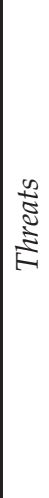 & $\begin{array}{l}\text { 1. Agree to disagree about } \\
\text { communication modalities and } \\
\text { technology options and move } \\
\text { forward with meeting JCIH } \\
\text { benchmarks. } \\
\text { 2. Increase family involvement by } \\
\text { training and paying parents to } \\
\text { provide family support. } \\
\text { 3. Implement a support program } \\
\text { that can help families navigate } \\
\text { systems and accept having a } \\
\text { child who is deaf or hard of } \\
\text { hearing. }\end{array}$ & $\begin{array}{l}\text { 1. Strengthen relationships with } \\
\text { agencies and organizations that } \\
\text { provide family support to enable } \\
\text { them to provide support to } \\
\text { families with children who are } \\
\text { deaf or hard of hearing. } \\
\text { 2. Create local family-to-family } \\
\text { outreach programs. } \\
\text { 3. Increase family involvement by } \\
\text { providing support for families. }\end{array}$ \\
\hline
\end{tabular}

$\mathrm{S}=$ Strength $\mathrm{W}=$ Weakness $\mathrm{O}=$ Opportunity $\mathrm{T}=$ Threat

utilizing national and state family resources help provide family support to families with infants newly identified with hearing loss. To overcome weaknesses, opportunities must be pursued (W-O strategy). For example, connecting families to other families who live in rural areas through traditional and electronic communication may increase support opportunities. Identifying ways to use strengths will ultimately reduce vulnerability to threats (S-T strategy). For example, affiliating state EHDI programs with national and local organizations may reduce costs associated with running family support services. Finally, establishing defensive plans will help to prevent weaknesses from making policies and services susceptible to threats (W-T strategy). For example, families may become more involved in their child's hearing health care needs if programs increase family-to-family outreach programs. 
From the 12 recommendations, 3 distinct strategies for improving family support services emerged. Specifically, states should: 1) develop and implement family support programs, 2) have paid staff in their programs who are parents of children who are deaf or hard of hearing, and 3) utilize appropriate materials created by national and local agencies and support groups.

\section{Limitations}

Limitations of this study include those inherent in the application of SWOT / TOWS analysis when applied to rapidly changing conditions. SWOT analysis is a subjective tool that explores conditions that are perceived rather than objectively measured. In this sense, the SWOT analysis is influenced by the perspectives of those who completed the surveys as well as those who analyzed the responses. Furthermore, SWOT analysis characterizes the situation at a specific point in time. In addition, SWOT analysis, as applied here, resulted in overlapping themes within a category. For example, funding could be identified as a strength, weakness, opportunity, or threat (see White \& Blaiser, 2011, for additional information regarding the selection of SWOT/TOWS for this study and further comment regarding interpretation and application of findings).

\section{Summary}

Family support as a component of EHDI brings numerous strengths and opportunities, as well as the potential for weaknesses and threats, to state EHDI programs. Planned programmatic support of families through the EHDI process (from screening through early intervention) could lead to improved followup outcomes (i.e., percentage of children who receive audiological evaluation and/or intervention services and earlier age of diagnosis) for individual EHDI programs. EHDI coordinators in states where formal family support mechanisms have been initiated or established indicated that their EHDI program derives significant benefit from these mechanisms. In addition, employing parents as EHDI staff, partnering with organizations or agencies that provide family support programming, and ensuring that families are aware of the many types and opportunities for family support are strategies that appear mutually beneficial to both families and EHDI programs. Further investigation is warranted to identify specific types and degrees of family support needed through EHDI programs to increase parental follow-up, such as audiological evaluation and enrollment in early intervention. Furthermore, the impact of family support on a family's acceptance of hearing loss and the long-term impact on a child's communication development are areas that require additional study.

When a child is referred for audiological evaluation after newborn hearing screening and is subsequently identified with hearing loss, the child's 
parents and caregivers need consistent and comprehensive family support. Linking state EHDI programs to national and local organizations is one way this support can be provided to families. Opportunities for families to meet, interact, and network with other families of children with hearing loss is associated with positive outcomes for the child as well as the parents and caregivers. Improving the well-being of the family as a unit provides a favorable environment to support the child's developmental and communicative success - a goal that remains at the center of all EHDI programs.

\section{Acknowledgement}

The work reported in this article was funded in part by the Maternal and Child Health Bureau under Cooperative Agreement \# U52MC04391 with the National Center for Hearing Assessment and Management at Utah State University. The opinions expressed in the article are those of the authors and do not necessarily reflect those of the Bureau.

\section{References}

Centers for Disease Control and Prevention (CDC). (2010). Identifying infants with hearing loss-United States, 1999-2007. Morbidity and Mortality Weekly Report, 59(8), 220-223.

Clark, K., \& Pittman, P. (2006, February). The role of early intervention programs in providing family support. Paper presented at the 2006 National Early Hearing Detection and Intervention Conference, Washington, D.C.

Jackson, C. W., Wegner, J. R., \& Turnbull, A. P. (2010). Family quality of life following identification of deafness. Language, Speech, Hearing Services in the School, 41(2), 194-205.

Joint Committee on Infant Hearing (JCIH). (2007). Year 2007 position statement: Principles and guidelines for early hearing detection and intervention programs. Pediatrics, 120(4), 898-921.

Hands \& Voices. (2011). Guide by your side. Retrieved May 25, 2011, from http:// www.handsandvoices.org/gbys/index.htm.

Kurtzer-White, E., \& Lutterman, D. (2003). Families and children with hearing loss: Grief and coping. Mental Retardation and Developmental Disabilities Research Reviews, 9, 232-235.

Moeller, M. P. (2000) Early intervention and language development in children who are deaf and hard of hearing. Pediatrics, 106(3), e43.

National Center for Hearing Assessment and Management (NCHAM). (2011). Culturally competent family support. Retrieved May 19, 2011, from http:// www.infanthearing.org/familysupport/index.html.

Nelson, H., Bougatsos, C., \& Nygren, P. (2008). Universal newborn hearing screening: Systematic review to update the 2001 U.S. Preventive Services Task Force recommendation. Pediatrics, 122(3), 266-276. 
Pipp-Siegel, S., Sedey, A., \& Yoshinaga-Itano, C. (2002). Predictors of parental stress in mothers of young children with hearing loss. Journal of Deaf Studies and Deaf Education, 7(1), 1-17.

Vohr, B. R., Jodoin-Krauzyk, J., Tucker, R., Johnson, M. J., Topol, D., \& Algren, M. (2008). Results of newborn screening for hearing loss: Effects on the family in the first 2 years of life. Archives Pediatric Adolescent Medicine, 162(3), 205-11.

Vohr, B. R., Letourneau, K. S., \& McDermott, C. (2001). Maternal worry about neonatal hearing screening. Journal of Perinatology, 21(1), 15-20.

White, K. R. (1997, October). The scientific basis for newborn hearing screening: Issues and evidence. Invited keynote address to the Early Hearing Detection and Intervention (EHDI) Workshop sponsored by the Centers for Disease Control and Prevention, Atlanta, Georgia.

White, K., \& Blaiser, K. (2011). Strategic planning to improve early hearing detection and intervention programs. The Volta Review, 111(2), 83-108.

Yoshinaga-Itano, C. (2003). From screening to the early identification and intervention: Discovering predictors to successful outcomes for children with significant hearing loss. Journal of Deaf Studies and Deaf Education, 8(1), $11-30$.

Yoshinaga-Itano, C (2004). Levels of evidence: Universal newborn hearing screening (UHNS) and early hearing detection and intervention systems (EHDI). Journal of Communication Disorders, 37(5), 451-465.

Yoshinaga-Itano, C., \& Abdala deUzcategui, C. (2001). Early identification and social-emotional factors of children with hearing loss and children screened for hearing loss. In E. Kurtzer-White \& D. Lutterman (Eds), Early Childhood Deafness, (p. 13-28). Baltimore, MD: York Press.

Yoshinaga-Itano, C., Sedey, A., Coulter, D., \& Mehl, A. (1998). Language of early-and-later identified children with hearing loss. Pediatrics, 102(5), 1161-71.

Young, A., \& Tattersall, H. (2007). Universal newborn hearing screening and early identification of deafness: Parent's responses to knowing early and their expectations of child communication development. Journal of Deaf Studies and Deaf Education, 12(2), 209-220. 
The Volta Review, Volume 111(2), Summer 2011, 195-208

\title{
Periodic Early Childhood Hearing Screening: The EHDI Perspective
}

\author{
Jeff Hoffman, M.S., CCC-A; \\ K. Todd Houston, Ph.D., CCC-SLP, LSLS Cert. AVT; \\ Karen F. Muñoz, Ed.D., CCC-A; and Tamala S. Bradham, Ph.D., CCC-A
}

State coordinators of early hearing detection and intervention (EHDI) programs completed a strengths, weaknesses, opportunities, and threats, or SWOT, analysis that examined 12 areas within state EHDI programs. Concerning periodic early childhood hearing screening, 47 coordinators listed 241 items and themes were identified within each SWOT category. A threats, opportunities, weaknesses, and strengths, or TOWS, analysis yielded 12 recommendations that focused on similar themes. Out of these 12 recommendations, 3 distinct strategies emerged: 1) increase education of parents and professionals about risk factors for later onset hearing loss and hearing monitoring; 2) integrate data systems accessible by professionals that are capable of processing risk factors and multiple hearing screenings; and 3) collaborate with early childhood programs to coordinate follow-up, improve data submission, and conduct periodic hearing screenings. Addressed appropriately, these recommendations can improve periodic early childhood screening within state EHDI programs.

\section{Introduction}

In 2007, early hearing detection and intervention (EHDI) programs in 43 states and territories reported that 1.2 out of every 1,000 newborns screened were diagnosed with a permanent childhood hearing loss (Centers for Disease

Jeff Hoffman, M.S., CCC-A, is a Senior Research Scientist - Audiologist in the National Center for Hearing Assessment and Management at Utah State University. K. Todd Houston, Ph.D., CCC-SLP, LSLS Cert. AVT, is an Assistant Professor in the Department of Communicative Disorders and Deaf Education at Utah State University. Karen F. Muñoz, Ed.D., CCC-A, is an Assistant Professor in the Department of Communicative Disorders and Deaf Education at Utah State University. Tamala S. Bradham, Ph.D., CCC-A, is an Assistant Professor in the School of Medicine, Department of Hearing and Speech Sciences, at Vanderbilt University. Correspondence concerning this manuscript should be directed to Mr. Hoffman at jeffhoffman.ehdi@gmail.com. 
Control and Prevention [CDC], 2010a). However, the prevalence of hearing loss nearly doubles by school age (Bamford, et. al., 2007). In addition, approximately $75 \%$ of children experience otitis media by the time they are 3 years old (National Institute on Deafness and Other Communication Disorders [NIDCD], 2010), and a mild conductive hearing loss may occur when middle ear fluid is present (Fria, Cantekin, \& Eichler, 1985).

In the United States, 97\% of newborns have their hearing screened, most during birth admission, using otoacoustic emissions (OAE) or automated auditory brainstem response (AABR) screening technology (CDC, 2010b). However, there is no comparable universal 2nd tier screening system to identify postnatal hearing loss during the early childhood years. Consequently, many young children may not have another hearing screening until long after the newborn period and in some cases not until school.

The American Academy of Pediatrics (AAP, 2008) recommends an objective hearing screening for newborns and 4 year olds, with a risk assessment and appropriate follow-up at periodic examinations between those ages. Medicaid's Early Periodic Screening, Diagnosis, and Treatment (EPSDT) program for children requires states to develop their own hearing screening periodicity schedule that meets "reasonable standards of medical practice" in consultation with recognized medical organizations (U.S. Department of Health and Human Services [DHHS], 2005).

Guidelines developed by the Joint Committee on Infant Hearing (JCIH, 2007) regarding hearing surveillance, screening, and evaluation after the neonatal period include:

- Monitoring auditory skills, middle-ear status (using pneumatic otoscopy and/or tympanometry), and developmental milestones consistent with the AAP periodicity schedule.

- Administering a validated global screening tool at age 9, 18, and 24-30 months, or sooner if there are parent or physician concerns about hearing abilities or language development.

- Referral to an audiologist and/or speech-language pathologist if the child does not pass the speech/language part of the screening; if there are physician or parent concerns about hearing, speech, or language; or if there are risk factors for later onset hearing loss.

The Part C early intervention program and the Part B preschool special education program, authorized by the Individuals with Disabilities Education Act (IDEA), is another system where hearing screening and evaluation occur for some young children. It is estimated that over $12 \%$ of young children have developmental delays and would, therefore, be eligible for Part $C$ early intervention services (Rosenberg, Zhang, \& Robinson, 2008). Both Part C and Part B programs require an evaluation for each child to determine eligibility for services. In a survey of these programs, Part $C$ programs reported that $28 \%$ of the 
children were screened using OAEs, a technique that screens the peripheral auditory system at the level of the cochlea; only $14 \%$ received a complete audiological evaluation (Eiserman \& Shisler, 2010). The use of family questionnaires and non-calibrated noisemakers, regarded as inappropriate according to existing guidelines for hearing screening (American Speech-LanguageHearing Association, 1997), were used more frequently. A higher percentage of 3- to 5-year-old children in Part B programs received objective screening and evaluation. Although only $17 \%$ received an audiological evaluation, $46 \%$ of the children were screened using pure tone audiometry and $22 \%$ were screened using OAEs (Eiserman \& Shisler, 2010).

The Early Head Start and Head Start (EHS/HS) program, with an annual enrollment of more than 900,000 children ages birth to 5 years (DHHS, 2010), is another program where children receive a hearing screening. A hearing screening within 45 days after enrollment into the EHS/HS program is required by the Head Start Performance Standards (DHHS, 2009). The Early Childhood Hearing Outreach $(\mathrm{ECHO})$ initiative at the National Center for Hearing Assessment and Management (NCHAM) began training EHS/HS programs in 2002 to update their hearing screening practices through the use of OAE screening and a specified follow-up protocol. More than $200 \mathrm{EHS} / \mathrm{HS}$ programs in 21 states have adopted the ECHO model, and more than 90,000 young children have been screened. Approximately 1.5 of every 1,000 young children in the EHS/HS program have been identified with a permanent hearing loss, and 18 per 1,000 have been identified with otitis media (Eiserman, Hartel, Shisler, Buhrman, White, \& Foust, 2008).

The purpose of this study was to complete a systematic review using a strengths, weaknesses, opportunities, and threats, or SWOT, and a threats, opportunities, weaknesses, and strengths, or TOWS, analysis to determine current EHDI practices related to periodic early childhood screening, and to identify recommendations to improve program effectiveness in this area.

\section{Methods}

Fifty-one state EHDI coordinators were asked to complete an online survey. Related to periodic early childhood screening programs, the survey asked the EHDI coordinator to report at least 1 item in the following four areas to assist with strategic planning: strength, weakness, opportunity, and threat. The responses obtained were reviewed by a panel of experts in the field and categorized into common themes in each of these four strategic planning areas. To generate recommendations from the SWOT analysis, a TOWS matrix was used to match identified strengths with opportunities (S-O strategy), strengths with threats (S-T strategy), weaknesses with opportunities (W-O strategy), and weaknesses with threats (W-T strategy). For an in-depth review of the methodology, the reader is referred to White and Blaiser, 2011. 


\section{Results}

Forty-seven state EHDI coordinators (92\%) completed information about periodic early childhood screening in the SWOT questionnaire. The respondents generated 68 items in the strength category, 62 items in the weakness category, 51 in the opportunity category, and 60 in the threat category for a total of 241 responses. Based on the responses, several specific themes were generated for each category: 9 for strengths, 7 for weaknesses, 7 for opportunities, and 7 threats (Table 1). Each category also had a miscellaneous section that included no comment, N/A, or items that did not fit within the themes identified.

Table I. Frequency of themes within each SWOT category $(\mathrm{n}=241)$

\begin{tabular}{|c|c|c|}
\hline SWOT Category & Themes & Frequency $n(\%)$ \\
\hline Strengths $(n=68)$ & $\begin{array}{l}\text { Protocols, Policies, and Procedures } \\
\text { Screening Outreach Efforts } \\
\text { Miscellaneous / No Comment } \\
\text { Awareness and Resources } \\
\text { Data Tracking System } \\
\text { Governance, Administration, and Personnel } \\
\text { Legislation } \\
\text { Funding } \\
\text { Professional Training }\end{array}$ & $\begin{aligned} 18(26 \%) \\
13(19 \%) \\
11(16 \%) \\
7(10 \%) \\
5(7 \%) \\
5(7 \%) \\
3(4 \%) \\
3(4 \%) \\
3(4 \%)\end{aligned}$ \\
\hline Weaknesses $(n=62)$ & $\begin{array}{l}\text { Not Provided through EHDI } \\
\text { Miscellaneous/No Comment } \\
\text { Protocols, Policies, and Procedures } \\
\text { Data Management and Tracking } \\
\text { Professional Education } \\
\text { Staffing Limitations } \\
\text { Legislation }\end{array}$ & $\begin{array}{l}14(23 \%) \\
13(21 \%) \\
12(19 \%) \\
11(18 \%) \\
6(10 \%) \\
4(6 \%) \\
2(3 \%)\end{array}$ \\
\hline Opportunities $(n=51)$ & $\begin{array}{l}\text { Miscellaneous/No Comment } \\
\text { Data Management and Tracking } \\
\text { Educate and Train Parents and Professionals } \\
\text { Collaboration } \\
\text { Protocols, Policies, and Procedures } \\
\text { Legislation } \\
\text { Equipment }\end{array}$ & $\begin{aligned} 14(27 \%) \\
11(22 \%) \\
10(20 \%) \\
8(12 \%) \\
6(13 \%) \\
1(2 \%) \\
1(2 \%)\end{aligned}$ \\
\hline Threats $(n=60)$ & $\begin{array}{l}\text { Funding } \\
\text { Miscellaneous/No Comment } \\
\text { Staff Limitations } \\
\text { Protocols, Policies, and Procedures } \\
\text { Educate and Train Parents and Professionals } \\
\text { Low Priority } \\
\text { Access to Appropriate Care }\end{array}$ & $\begin{array}{l}18(30 \%) \\
16(27 \%) \\
11(18 \%) \\
7(12 \%) \\
5(8 \%) \\
2(3 \%) \\
1(2 \%)\end{array}$ \\
\hline
\end{tabular}




\section{Strength Characteristics}

The three most frequently cited strengths were protocols, policies, and procedures $(26 \%)$, screening outreach efforts $(19 \%)$, and awareness and resources $(10 \%)$ (Table 2). Twenty-six percent of respondents reported that their state had policies and procedures that focused on following up with children considered at risk for later onset or progressive hearing loss. Respondents also noted that they notify families directly about the risk factors, contact the infant's primary health care provider, work with the audiologist in an ongoing process, and coordinate with the early intervention system in their state to recommend and/or arrange for additional hearing screening and monitoring. In addition, one program indicated that the state has the capacity to collect data for children up to 6 years old. Another EHDI program has a state-wide outpatient hearing screening initiative.

Screening outreach efforts were also identified by $19 \%$ of respondents as a strength. Hearing screenings in community programs, such as EHS/HS, early intervention programs, Parent As Teachers organizations, early care and education settings, and health clinics (public and community), were all mentioned. One state EHDI coordinator indicated having 42 screening sites across the state with the capacity for OAE, immittance, pure tone audiometry, and otoscopy.

Table 2. Top three themes for strengths

Themes

Protocols, Policies, and Procedures

Screening Outreach Efforts

Awareness and Resources
Descriptions

State programs have systems in place to follow-up with families, audiologists, and physicians on infants identified with risk factors (e.g., reminder letters, fact sheets).

State programs collect data on children through age 6.

Audiologists actively track the infants with risk factors.

Local health departments, hearing coordination centers, Early Head Start programs, or Parents as Teachers programs provide hearing screenings throughout the state.

Legislation that mandates all day care centers to provide annual hearing and vision screenings.

Provide state-wide trainings in hospitals.

Developed brochures and posters on JCIH recommendations to disseminate to medical homes and audiologists.

Educating the neonatal intensive care units to provide information to families. 
Increasing the awareness of later onset hearing loss and resources was viewed as a strength by $10 \%$ of respondents. Specifically, EHDI coordinators indicated that education of professionals about risk factors for later onset and progressive hearing losses has increased awareness and encouraged parents to monitor their child's hearing. The development of materials specifically for birthing facilities, primary care practitioners, and/or audiologists on periodic early childhood hearing screening was also mentioned.

Other areas identified as strengths included data tracking systems $(7 \%)$, governance, administration, and personnel $(7 \%)$, legislation (4\%), funding $(4 \%)$, and professional training $(4 \%)$. Data tracking systems that were capable of recording risk factors for later onset and progressive hearing losses were considered to be a strength. One respondent indicated that the EHDI data system contributed to child health records. Program factors that strengthened periodic early childhood hearing screening were the availability of staff to follow-up and the organizational structure of the EHDI program. One EHDI coordinator listed having trained screening personnel as an advantage to support periodic early childhood hearing screening.

\section{Weakness Characteristics}

The three most frequently cited weaknesses were periodic early childhood hearing screenings not provided through the EHDI system (23\%), protocols, policies, and procedures $(19 \%)$, and data management and tracking $(18 \%)$ (Table 3). Twenty-three percent of respondents reported that their

Table 3. Top three themes for weaknesses

\begin{tabular}{|c|c|}
\hline Themes & Descriptions \\
\hline Not Provided through EHDI & $\begin{array}{l}\text { EHDI does not provide periodic hearing screenings- } \\
\text { hope someone else it is doing it. } \\
\text { EHDI does not track at-risk children. } \\
\text { Hearing screenings for infants and toddlers with risk } \\
\text { factors is not universally implemented in the state. }\end{array}$ \\
\hline $\begin{array}{l}\text { Protocols, Policies, and } \\
\text { Procedures }\end{array}$ & $\begin{array}{l}\text { Lack of reporting by providers. } \\
\text { Confusion regarding what risk factors require } \\
\text { follow-up hearing screenings and when. } \\
\text { Protocols are not universally implemented across all } \\
\text { sites. }\end{array}$ \\
\hline $\begin{array}{l}\text { Data Management and } \\
\text { Tracking }\end{array}$ & $\begin{array}{l}\text { No data system to track at-risk children. } \\
\text { Families move, change names, or report inaccurate } \\
\text { information making it difficult to track long term. } \\
\text { Need a system that will generate letters every } 6 \\
\text { months until the age of } 3 \text { to send to families and } \\
\text { the medical homes. }\end{array}$ \\
\hline
\end{tabular}


EHDI program does not provide periodic early childhood hearing screenings and, if administered, it is scattered and inconsistent throughout the state.

Protocols, policies, and procedures were identified as a weakness by $19 \%$ of EHDI coordinators. Specifically, coordinators listed the inconsistent identification and reporting of risk factors for later onset or progressive hearing loss; periodic hearing screening was not routinely completed because re-screening was not conducted at the recommended ages or because patients did not return for the appointment; and failure to report risk factors or screening results for this age group to the state EHDI program.

Issues with data tracking and management were also noted as a significant weakness by $18 \%$ of respondents. Factors contributing to loss to follow-up from newborn hearing screening (e.g., changing addresses, phone numbers, and primary care physicians) prohibited some respondents from implementing an early childhood hearing screening program. Some coordinators reported that the limited capacity of the state EHDI data system to gather and process results for this age group was also a weakness. Many EHDI coordinators also indicated that they do not have the data management system to track this population.

Other identified weaknesses include professional education (10\%), staffing limitations (6\%), and legislation (3\%). Specifically, EHDI coordinators reported that physicians did not know the risk factors for hearing loss, how to refer for follow-up testing, and had a lack of buy-in to monitor hearing. Staff limitations included a shortage of providers who accept Medicaid, a lack of staff follow-up for missed appointments, and excessive time spent on data entry, which prevented focus on periodic early childhood hearing screenings. EHDI coordinators also reported that the lack of a legislative mandate on reporting risk factors and delayed onset/progressive hearing losses was considered a weakness.

\section{Opportunities for EHDI Programs}

The three most frequently cited opportunities were data management systems $(22 \%)$, education and training of parents and professionals $(20 \%)$, and collaboration (12\%) (Table 4). Related to data management systems, $22 \%$ of EHDI coordinators reported either developing or modifying their existing data management system to include risk factor variables, progressive hearing losses variables, and periodic early childhood hearing screening results as a child ages. Integrating or linking data management systems to community providers (e.g., audiologists, primary care physicians) were also listed as opportunities. In addition, some respondents were considering integrating automatic reporting of when a child should have another hearing screening into the data management system.

Twenty percent of respondents reported opportunities to educate and train parents and professionals in periodic early childhood hearing screening. 
Table 4. Top three themes for opportunities

\begin{tabular}{lc}
\hline Themes & Descriptions \\
\hline Data Management and Tracking & $\begin{array}{c}\text { Will be modifying the existing data management } \\
\text { system to track this population. } \\
\text { Integrating data management systems will allow } \\
\text { for alerts to pediatrician's offices automatically } \\
\text { and electronically. }\end{array}$ \\
$\begin{array}{c}\text { Educate and Train Parents and } \\
\text { Professionals }\end{array}$ & and families. \\
& Promoting developmental screenings in pediatric \\
& offices. \\
Building community awareness and capacity to \\
provide trainings on hearing screenings. \\
EHDI and early intervention are in the same \\
department/division/agency. \\
Working closely with Head Start, public health \\
department nurses, and/or the ECHO project. \\
Connecting with stakeholders at meetings and \\
conferences.
\end{tabular}

Specifically, EHDI coordinators indicated they had opportunities to provide training to physicians and medical homes about JCIH guidelines, the importance of hearing and developmental screenings, and the impact of unidentified hearing loss. They were also reaching out to families to educate them on risk indicators for hearing loss.

Collaboration, especially with programs that were beginning to conduct OAE hearing screenings throughout early childhood, was an opportunity noted by $12 \%$ of the EHDI coordinators. Items listed under this theme included the ECHO initiative with EHS/HS programs and better collaboration with primary care physicians through the AAP, as well as Parents As Teachers and early intervention programs.

Protocols, policies, and procedures (13\%) offered opportunities for some EHDI programs to further develop periodic early childhood hearing screening. Using the NICHQ's Learning Collaborative project, respondents indicated that their programs have either revised or will be revising their protocols and procedures. Other opportunities included adding risk factors to the dried blood spot filter paper test, re-structuring short-term follow-up, establishing timelines for screening, and sending reminder letters to families and the medical home/primary care physician.

Other opportunities mentioned by EHDI coordinators included mandating reporting of risk factors and all screening/test results for children birth to 3 years of age $(2 \%)$, and the provision of hearing screening equipment for usage by field staff to follow this population $(2 \%)$. 
The three most frequently cited threats were funding (30\%), staff limitations (18\%), and protocols, policies, and procedures (12\%) (Table 5). Of note, $27 \%$ of the respondents had no comment or did not know of any threats to periodic early childhood hearing screening. Related to funding, 30\% of EHDI coordinators expressed concerns regarding financial constraints, budget cuts, reduction in health care coverage, an increase in the uninsured population, and inadequate funding to support tracking children at risk for hearing loss or later onset of hearing loss.

Eighteen percent of respondents noted staff limitations as a threat, including a lack of resources, time constraints to continuing the ECHO project, staff turnover, and multiple data system tracking results. Twelve percent of respondents identified non-compliance with the recommended monitoring guidelines, inability to track families over a 3-year period due to relocations and/or name changes, and lack of documentation as threats to protocols, policies, and procedures. Respondents also noted that the modification of screening guidelines by the JCIH (from monitoring every 6 months to one evaluation prior to 30 months; 2007) increased the likelihood of not maintaining contact with the family.

Difficulty in educating and training parents and professionals about monitoring hearing through periodic screening was identified by $8 \%$ of EHDI coordinators. Specifically, coordinators expressed difficulty in reaching a sufficient number of primary care physicians through training efforts, a concern that parents may not understand the role of hearing in their children's development, and difficulty educating parents to follow through on recommendations. Additional threats included periodic early childhood hearing screenings considered a low priority by parents and primary care physicians $(3 \%)$ and access to appropriate care (2\%).

Table 5. Top three themes for threats

\begin{tabular}{|c|c|}
\hline Themes & Descriptions \\
\hline Funding & $\begin{array}{l}\text { Increase in the uninsured population. } \\
\text { Budget cuts. } \\
\text { Periodic hearing screening programs are not self-sustainable } \\
\text { and may have to end. }\end{array}$ \\
\hline Staff Limitations & $\begin{array}{l}\text { Lack of staff/resources in EHDI to direct toward this area. } \\
\text { Time constraints. } \\
\text { Turnover in community programs leads to loss of momentum. }\end{array}$ \\
\hline $\begin{array}{l}\text { Protocols, Policies, and } \\
\text { Procedures }\end{array}$ & $\begin{array}{l}\text { Medical homes are noncompliant with } \\
\text { monitoring recommendations by JCIH. } \\
\text { Protocols are not being followed by providers. } \\
\text { No protocols developed to address family issues (i.e., families } \\
\text { relocating, changing names, do not respond, etc.). }\end{array}$ \\
\hline
\end{tabular}




\section{TOWS Analysis Matrix}

By applying the TOWS analysis matrix to the themes identified in the SWOT analysis, specific recommendations, or "strategic options," were derived from the internal and external environmental factors identified by the EHDI coordinators. By systematically pairing the four categories of the SWOT analysis, recommendations can be derived to maximize resources and effect positive change. Based on the SWOT themes, 12 individual recommendations were made (Table 6). To maximize opportunities, identification of strengths can lead to positive change (S-O strategy). For example, increasing collaboration with early childhood programs serving young children, ages birth to 3-years-old, can help strengthen follow-up efforts and improve data submission. By pursuing opportunities, weaknesses can be minimized (W-O strategy). For example, increasing educational efforts for primary care physicians about risk factors identified in the JCIH 2007 position statement and the recommended monitoring for hearing loss when risk factors are present. Identification of ways to use strengths can ultimately reduce vulnerability to threats (S-T strategy). For example, reduce follow-up staff workload by modifying existing data systems to efficiently generate reminders to primary health care providers and families of hearing monitoring screenings/evaluations due to risk factors. Finally, establishing defensive plans will help to prevent weaknesses from making programs susceptible to threats (W-T strategy). For example, develop data sharing agreements to increase the reporting of risk factors and 2nd tier hearing screenings from a variety of providers. Out of these 12 recommendations, 3 distinct strategies emerged for improving periodic early childhood screening. Specifically, states should: 1) increase education of parents and professionals about risk factors for later onset hearing loss and hearing monitoring; 2) integrate data systems accessible by professionals that are capable of processing risk factors and multiple hearing screenings; and 3) collaborate with early childhood programs to coordinate follow-up, improve data submission, and conduct periodic hearing screenings.

\section{Limitations}

Limitations of this study include those inherent in the application of SWOT / TOWS analysis when applied to rapidly changing conditions. SWOT analysis is a subjective tool that explores conditions that are perceived rather than objectively measured. In this sense, the SWOT analysis is influenced by the perspectives of those who completed the surveys as well as those who analyzed the responses. Furthermore, SWOT analysis characterizes the situation at a specific point in time. In addition, SWOT analysis, as applied here, resulted in overlapping themes within a category. For example, protocols, policies, and procedures could be identified as a strength, weakness, opportunity, or threat. Similarly, themes regarding data systems and education/training 
Table 6. TOWS analysis matrix for periodic early childhood screening

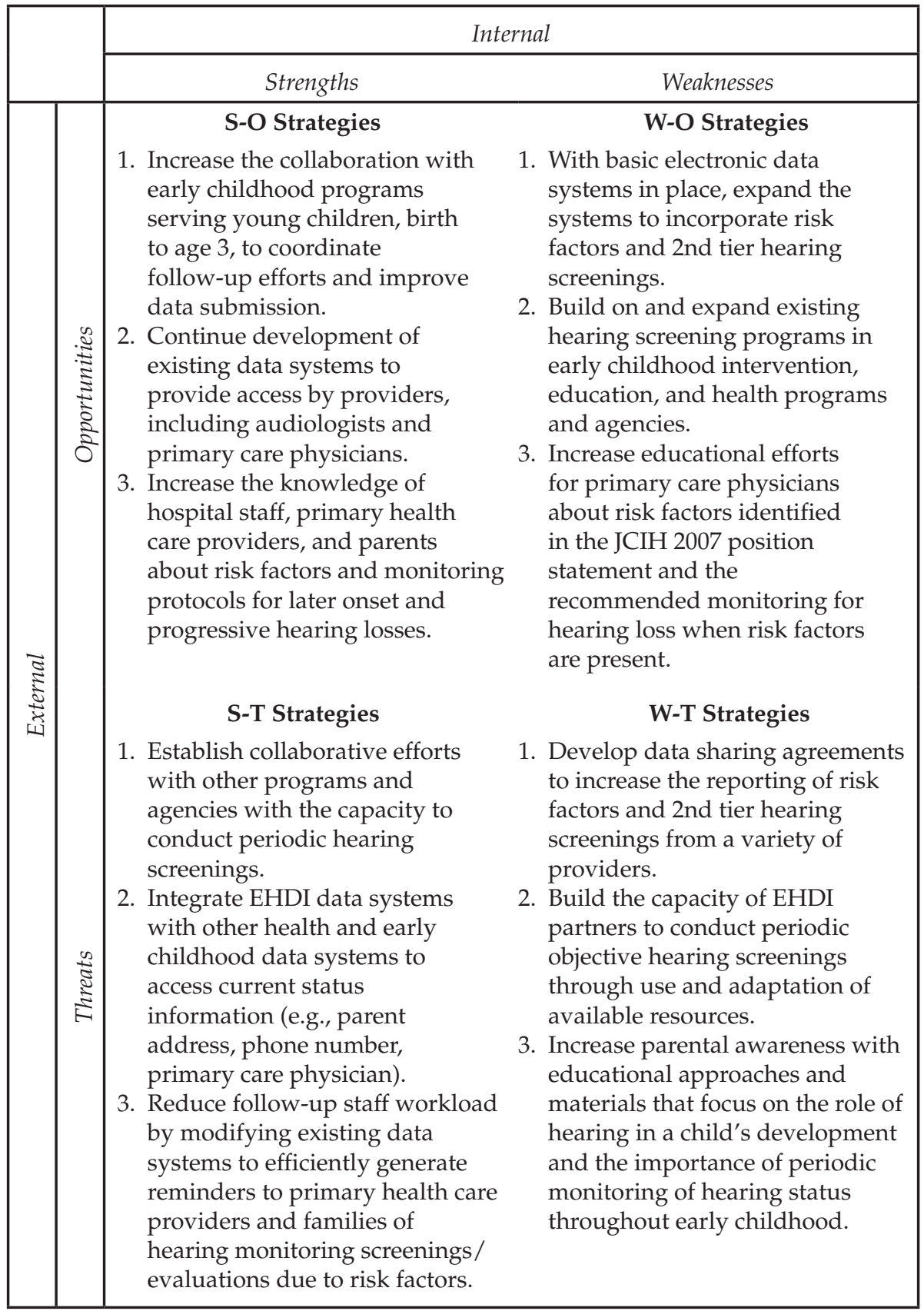

$\mathrm{S}=$ Strength $\mathrm{W}=$ Weakness $\mathrm{O}=$ Opportunity $\mathrm{T}=$ Threat 
were identified in three of the four categories (see White \& Blaiser, 2011, for additional information regarding the selection of SWOT/TOWS for this study and further comment regarding interpretation and application of findings).

\section{Summary}

Programs are at different levels of developing the various components of the EHDI system. Some programs have been in existence for nearly 20 years, while others are barely a decade old. Some do not have state legislation and regulations, while others do; however, the statutes and regulations vary among those programs. Some programs have received federal funding to develop the capacity of their EHDI systems and data systems, although length of funding and funding amounts have varied. It should not be surprising that programs are on a continuum of experience with regard to periodic early childhood hearing screening. This is reflected in the degree to which EHDI data systems in different states are capable of recording and tracking risk factors for later onset and progressive hearing losses as well as hearing screening beyond the newborn period.

It is also not surprising that much of the focus of this area was on identification, reporting, and follow-up with risk factors for later onset or progressive hearing loss since risk factors have been addressed in the position statements of the JCIH since 1973 (JCIH, 1973). To a lesser extent, the more recent focus on screening and surveillance for hearing loss in the medical home (JCIH, 2007) is reflected in fewer comments regarding more routine periodic screening for hearing loss throughout early childhood. In the periodic hearing screening area of the SWOT analysis, a number of EHDI coordinators had "no comment," or provided comments that were highly individualized or tangential to the area and thus classified as "miscellaneous." The percentage of EHDI coordinators with "miscellaneous, or no comment" items in each area were: strengths $-16 \%$; weaknesses $-21 \%$; opportunities $-27 \%$; and threats $-27 \%$.

In summarizing the SWOT and TOWS analyses based on the 241 items contributed by the EHDI coordinators, the following global recommendations emerge regarding periodic early childhood screening:

- Education for providers, including hospital staff, audiologists, and, especially, primary care physicians, should focus on the risk factors for later onset and progressive hearing loss and recommended monitoring protocols. Approaches to increasing parental awareness of risk factors for hearing loss, the role of hearing in the child's development, and the importance of periodic hearing screening throughout early childhood should be further developed.

- Data systems need ongoing development to allow access to a child's hearing health record by primary care physicians and audiologists, to allow the reporting of risk factors, hearing screening, and evaluation results by 
health and intervention providers based on data sharing agreements, to link or integrate with other child health and intervention data systems, and to efficiently generate reminders for providers and parents consistent with a recommended protocol.

- Collaboration is needed with health, intervention, and educational programs that provide services to young children to coordinate follow-up efforts, to improve data submission, and to conduct periodic objective hearing screenings after being trained with available resources.

Finally, the degree to which the "early adopters" - those states that were among the first to begin implementing specific activities and now view these as strength areas - can share their experiences, both positive and negative, with programs that are just beginning to consider working in a particular area may facilitate the development of effective and efficient systems at the local, state, and national levels.

\section{Acknowledgements}

The work reported in this article was funded in part by the Maternal and Child Health Bureau under Cooperative Agreement \# U52MC04391 with the National Center for Hearing Assessment and Management at Utah State University. The opinions expressed in the article are those of the authors and do not necessarily reflect those of the Bureau.

\section{References}

American Academy of Pediatrics (AAP). (2008). Recommendations for preventative pediatric health care. Retrieved May 19, 2011, from http:/ /aappolicy. aappublications.org/cgi/content/full/pediatrics;105/3/645\#F1.

American Speech-Language-Hearing-Association (ASHA). (1997). Guidelines for audiologic screening. Retrieved May 19, 2011, from http:/ /www.asha.org/ docs/html/GL1997-00199.html.

Bamford, J., Fortnum, H., Bristow, K., Smith, J., Vamvakas, G., Davies, L., et al. (2007). Current practice, accuracy, effectiveness and cost-effectiveness of the school entry hearing screen. Health Technology Assessment, 11(32), 1-167.

Centers for Disease Control and Prevention (CDC). (2010a). Summary of diagnosis and loss to follow-up/loss to documentation (Year 2007). Retrieved May 19, 2011, from http:/ / www.cdc.gov/ncbddd/hearingloss/data.html.

Centers for Disease Control and Prevention (CDC). (2010b). Identifying infants with hearing loss - United States, 1999-2007. Morbidity and Mortality Weekly Report, 59(8), 220-223.

Eiserman, W., \& Shisler, L. (2010). What NHS programs can offer second tier hearing screening programs. Presented at the Newborn Hearing Screening 2010 Conference, Como, Italy. 
Eiserman, W., Hartel, D., Shisler, L., Buhrman, J., White, K., \& Foust, T. (2008). Using otoacoustic emissions to screen for hearing loss in early childhood settings. International Journal of Pediatric Otorhinolaryngology, 72(4), 475-482.

Fria, T., Cantekin, E., \& Eichler, J. (1985). Hearing acuity of children with otitis media with effusion. Archives of Otolaryngology - Head \& Neck Surgery, 111(1), 10-16.

Joint Committee on Infant Hearing (JCIH). (1973). Joint statement on neonatal screening for hearing impairment. Retrieved May 19, 2011, from http:/ /www. jcih.org/JCIH1971.pdf.

Joint Committee on Infant Hearing (JCIH). (2007). Year 2007 position statement: Principles and guidelines for early hearing detection and intervention programs. Pediatrics, 120(4), 898-921.

National Institute on Deafness and Other Communication Disorders (NIDCD). (2010). Ear infections in children. Retrieved May 19, 2011, from http://www. nidcd.nih.gov/health/hearing/earinfections.htm.

Rosenberg, S., Zhang, D., \& Robinson, C. (2008). Prevalence of developmental delays and participation in early intervention services for young children. Pediatrics, 121(6), e1503-e1509.

U.S. Department of Health and Human Services (DHHS). (2005). EPSDT benefits. Retrieved May 20, 2011, from http://www.cms.gov/ MedicaidEarlyPeriodicScrn/02_Benefits.asp.

U.S. Department of Health and Human Services (DHHS). (2009). Head start program performance standards; 45 CFR Chapter XIII, $\$ 1304.20$ (b) (1). Retrieved June 19, 2010, from http://eclkc.ohs.acf.hhs.gov/hslc/Head\%20Start $\% 20$ Program / Program \%20Design \%20and \%20Management/Head \%20 Start\%20Requirements/Head\%20Start\%20Requirements/1304.

U.S. Department of Health and Human Services (DHHS). (2010). Head start program fact sheet fiscal year 2010. Retrieved May 20, 2011, from http:// eclkc.ohs.acf.hhs.gov/hslc/Head\%20Start\%20Program/Head\%20Start\%20 Program\%20Factsheets/fHeadStartProgr.htm.

White, K., \& Blaiser, K. (2011). Strategic planning to improve early hearing detection and intervention programs. The Volta Review, 111(2), 83-108. 
The Volta Review, Volume 111(2), Summer 2011, 209-223

\title{
Professional Development: Are We Meeting the Needs of State EHDI Programs?
}

\author{
K. Todd Houston, Ph.D., CCC-SLP, LSLS Cert. AVT; \\ Karen F. Muñoz, Ed.D., CCC-A; and Tamala S. Bradham, Ph.D., CCC-A
}

State coordinators of early hearing detection and intervention (EHDI) programs completed a strengths, weaknesses, opportunities, and threats, or SWOT, analysis that consisted of 12 evaluative areas of EHDI programs. For the professional development area, 47 coordinators responded with a total of 223 items, and themes were identified in each SWOT category. A threats, opportunities, weaknesses, and strengths, or TOWS, analysis yielded 12 recommendations that focused on similar themes. Out of these 12 recommendations, 3 distinct strategies emerged: 1) target personnel shortages through collaborative relationships and partnerships by providing a range of professional development activities that target medical, clinical, and educational professionals who deliver direct services to young children with hearing loss and their families; 2) establish policies and procedures to eliminate barriers to training; and 3) publicize all training opportunities to foster greater participation. Addressed successfully, these recommendations can strengthen professional development in state EHDI programs.

\section{Introduction}

Hearing screening, diagnosis, and intervention require a continuum of services involving professionals from a range of disciplines. The Joint Committee on Infant Hearing $(\mathrm{JCIH})$ describes successful early hearing detection and intervention (EHDI) programs as having highly skilled professionals whose

K. Todd Houston, Ph.D., CCC-SLP, LSLS Cert. AVT, was an Assistant Professor in the Department of Communicative Disorders and Deaf Education at Utah State University at the time of this study and is currently at The University of Akron. Karen F. Muñoz, Ed.D., CCC- $A$, is an Assistant Professor in the Department of Communicative Disorders and Deaf Education at Utah State University. Tamala S. Bradham, Ph.D., CCC-A, is an Assistant Professor in the School of Medicine, Department of Hearing and Speech Sciences at Vanderbilt University. Correspondence concerning this manuscript may be directed to Dr. Houston at ktodd.houston@gmail.com. 
roles and responsibilities are well-defined and clearly understood (2007). Accordingly, essential EHDI team members include the birth hospital, pediatricians or primary health care professionals (e.g., the medical home), audiologists, otolaryngologists, speech-language pathologists, educators of children who are deaf or hard of hearing, and other early intervention professionals involved in delivering EHDI services (American Speech-Language-Hearing Association [ASHA], 2004, 2008; JCIH, 2007). Depending on the etiology of the hearing loss and other developmental and familial needs, additional professionals may have a role in delivering direct services to the child and/or family.

There is growing evidence of a critical shortage of professionals with the qualifications needed to deliver appropriate, evidence-based medical, clinical, and early intervention services to young children with hearing loss and their families (JCIH, 2007; Moeller, White, \& Shisler, 2006; Shulman, Besculides, Saltzman, Ireys, \& White, 2010; White, 2008).

\section{Starting at the Beginning: The Birth Hospital}

In the EHDI system, the birth hospital is a key component of the team (JCIH, 2007). The screening program is housed within the hospital and national standards and procedures have been established to ensure that all hearing screening is performed appropriately and that results are communicated in a sensitive manner to the parents. When hearing screening results mandate a referral for further diagnostic testing, the child's parents or caregivers need information and resources. In most hospitals, however, one or more professionals may conduct the hearing screening, including audiologists, physicians, nursing staff, trained volunteers, or technicians (White, 2008). Ensuring that all professionals are following established, evidenced-based screening procedures is the responsibility of the EHDI program coordinator. While some states have specific licensure requirements that govern the personnel who can conduct hearing screening, other states have no specific regulatory requirements. As White states, "regardless of who does the screening, [screeners] must be properly trained and supervised, and data should be kept on each screener's performance to enable timely and appropriate training and assistance when needed" (2008, p. 36). Thus, a commitment to quality control within the screening process is required by program coordinators and those professionals conducting the screenings.

\section{Pediatricians and Family Physicians}

The American Academy of Pediatrics Medical Home Initiatives (2002) recommends that pediatricians and family physicians work cooperatively with parents or caregivers and other allied health providers to establish the infant's medical home. A medical home is defined as an approach to providing health 
care services where care is accessible, family-centered, continuous, comprehensive, coordinated, compassionate, and culturally competent (JCIH, 2007). The primary health care provider acts in partnership with parents in a medical home to identify and access appropriate audiology, intervention, and consultative services needed to develop a global plan of appropriate and necessary health and (re)habilitative care for infants identified with hearing loss and infants at risk for hearing loss ( $\mathrm{JCIH}, 2007)$. While the primary care physician's role is clearly defined within the medical home, evidence exists of knowledge gaps among many pediatricians and family physicians regarding the EHDI system and their roles in providing care to young children with hearing loss. For example, Moeller et al. (2006) conducted a national survey of physicians ( $n=1,968$ responses) and found a high level of support for universal newborn hearing screening. In this study, $81.6 \%$ of physicians indicated that it was very important to screen all newborns at birth. However, Moeller et al. also identified several areas of professional development and continuing medical education deficiencies, including: 1) understanding follow-up stages after hearing screening and diagnosis, 2) knowing when and where to refer infants for follow-up procedures, 3) understanding the genetics of hearing loss, 4) implementing surveillance for late-onset hearing loss, 5) cochlear implantation and candidacy criteria, 6) contacts for referral to early intervention, 7) appropriate communication options, and 8) professional disciplines involved in early intervention. As recommended in this study, "additional education of the medical community on these issues will put physicians in a better position to support families of newly identified infants with permanent hearing loss" (Moeller, et al., 2006, p. 1365).

\section{Audiologists}

Audiologists play a vital role in the EHDI process. They are responsible for diagnosing hearing loss and fitting appropriate hearing technology. Once a newborn is referred from newborn hearing screening, an audiologist must either confirm or rule out the hearing loss. If a hearing loss is diagnosed and if the family is in favor of listening and spoken language, amplification is usually recommended. Audiologists may also provide newborn hearing screening, program development, management, quality assessment, and service coordination as well as audiological diagnosis, treatment, and management (JCIH, 2007). While the audiologist's role is essential to EHDI programs, challenges exist to ensure infants who do not pass a hearing screen are evaluated by well-qualified pediatric audiologists. Shulman et al. (2010) surveyed 55 universal newborn hearing screening and intervention programs and found that nearly half cited a lack of pediatric audiologists as a major obstacle to diagnostic evaluations. Furthermore, the same study postulated that the shortage of pediatric audiologists stems from at least three factors: 1) a lack of university training programs that emphasize pediatric audiology, 
2) the difficulty involved in evaluating infants due to the need for specialized equipment and extra time for testing, and 3) poor reimbursement for audiological diagnostic services and payment rates by both public and private insurance systems. To improve services, Roush states, "the advocacy and commitment that enabled the successful nationwide implementation of newborn hearing screening is now needed on behalf of personnel preparation in pediatric audiology" (2010, p. 217).

\section{Early Interventionists}

After a child is diagnosed with hearing loss, federal guidelines stipulate that a referral should be made to an early intervention program and services should be initiated as soon as possible after diagnosis of hearing loss but no later than 6 months of age (JCIH, 2007). Studies indicate that when children with hearing loss are identified early and intervention initiated before 6 months of age, they achieve language, speech, and social-emotional outcomes that are significantly better than children who are identified late (Apuzzo \& Yoshinaga-Itano, 1995; Mayne, Yoshinaga-Itano, \& Sedey, 1998; Pipp-Siegel, Sedey, VanLeeuwen, \& Yoshinaga-Itano, 2003; Yoshinaga-Itano, 2001).

Early intervention services providers may come from a variety of academic disciplines $(\mathrm{JCIH}, 2007)$, but speech-language pathologists and teachers of the deaf are typically the professionals who deliver direct services focused on communication development and academic achievement to children with hearing loss and their families (ASHA, 2004, 2008). For more than a decade, successful outcomes for children identified early with hearing loss have been tied to the services delivered by professionals who are well-trained in parent-infant intervention (Calderon, 2000; Moeller, 2000; Yoshinaga-Itano, Sedey, Coulter, \& Mehl, 1998). However, access to highly skilled practitioners, especially speech-language pathologists and teachers of the deaf, remains a challenge for most families. As numerous studies indicate, many practicing speech-language pathologists and teachers of the deaf have significant disparity in their professional training and are often unable to meet the current communication and educational needs of children with hearing loss (Compton, Tucker, \& Flynn, 2009; Houston \& Caraway, 2010; Houston \& Perigoe, 2010a; Johnson, 2004; Lenihan, 2010; Luckhurst, 2008; Robbins \& Caraway, 2010; Vernon, 2007).

This dearth of appropriately trained early interventionists has led many children with hearing loss and their families to be either underserved or not served at all. Shulman et al. (2010) found that one-third of the EHDI programs they surveyed $(n=55)$ reported a lack of early intervention services as a major problem. Historically, most children with hearing loss were not identified until 2 to 3 years of age (White, Forsman, Eichwald, \& Muñoz, 2010); thus, many early intervention programs are insufficiently staffed to handle the increased 
number of younger children identified through universal newborn hearing screening. As White et al. (2010) note, in states where newborn hearing screening programs have been effectively implemented, the average age at which children with hearing loss are identified has been reduced to an average of 2 to 3 months of age. Thus, it is apparent that there is a shortage of early interventionists who can provide services, and those who are currently working may not have the necessary background, knowledge, and skills to meet the communicative and developmental needs of infants and young children with hearing loss.

White (2007) noted several factors contributing to the lack of appropriate early intervention and early educational services for children with hearing loss and their families. There is a scarcity of health care and educational professionals who are trained and knowledgeable about effectively educating children with hearing loss, and a lack of inservice training programs for individuals already in the field and preservice programs for individuals entering the field (White, 2007). The JCIH (2007) challenges universities to develop specialty-track, interdisciplinary professional education programs at the preservice level and additional training opportunities for inservice practitioners that focus on the developmental, communicative, and educational needs of young children with hearing loss and their families. Addressing practitioners as well as university administrators and faculty, Houston and Perigoe (2010b, p. 340) summed up the situation, "what is clear is that professional preparation is closely linked to the quality of services for children with hearing loss and their families. If we keep our focus on what is best for children and families, then we cannot fail to continue in the right direction."

The purpose of this study was to complete a systematic review of professional development initiatives using a strengths, weaknesses, opportunities, and threats, or SWOT, and a threats, opportunities, weaknesses, and strengths, or TOWS, analysis to determine current EHDI practices in professional development, and identify factors to improve program effectiveness and lead to better outcomes for infants who are served by EHDI programs.

\section{Methods}

Fifty-one EHDI coordinators were asked to complete an online survey. Related to professional development, the survey asked the EHDI coordinator to report at least 1 item in the following four areas to assist with strategic planning: strength, weakness, opportunity, and threat. The responses were reviewed by a panel of experts in the field and categorized into common themes in each of these four strategic planning areas. To generate recommendations from the SWOT analysis, a TOWS matrix was used to match identified strengths with opportunities (S-O strategy), strengths with threats (S-T strategy), weaknesses with opportunities (W-O strategy), and weaknesses with threats (W-T strategy). For an in-depth review of the methodology, the reader is referred to White and Blaiser, 2011. 


\section{Results}

Forty-seven EHDI coordinators (92\%) completed the professional development area of the SWOT questionnaire. The respondents generated 59 items in the strengths category, 60 items in the weaknesses category, 51 items in the opportunities category, and 53 items in the threats category for a total of 223 responses. Based on these responses, several specific themes were generated for each category: 6 for strengths, 10 for weaknesses, 5 for opportunities, and 7 for threats (Table 1). Each category also had a miscellaneous section that included no comment, N/A, or items that did not fit within the themes identified.

Table I. Frequency of themes within each SWOT category $(n=223)$

\begin{tabular}{|c|c|c|}
\hline SWOT category & Themes & Frequency $n(\%)$ \\
\hline Strengths $(n=59)$ & $\begin{array}{l}\text { Professional/Personnel Development \& } \\
\quad \text { Training Provided } \\
\text { Funding } \\
\text { Collaboration/Relationships } \\
\text { Communication } \\
\text { Miscellaneous } \\
\text { EHDI Programs/Services/Current Practices }\end{array}$ & $\begin{array}{l}8(14 \%) \\
6(10 \%) \\
4(7 \%) \\
4(7 \%) \\
3(5 \%)\end{array}$ \\
\hline Weaknesses $(n=60)$ & $\begin{array}{l}\text { Lack of Perceived Need for Training } \\
\text { Personnel Shortage } \\
\text { Miscellaneous/No Comments } \\
\text { Barriers of Obtaining Training } \\
\text { Need/Lack of Professional Development \& } \\
\text { Training } \\
\text { Funding } \\
\text { Lack of Access to Trainings } \\
\text { Lack of Time for Training } \\
\text { Resources } \\
\text { Unable to Travel to Trainings }\end{array}$ & $\begin{array}{r}11(18 \%) \\
9(15 \%) \\
8(13 \%) \\
7(12 \%) \\
6(10 \%) \\
5(8 \%) \\
4(7 \%) \\
4(7 \%) \\
4(7 \%) \\
2(3 \%)\end{array}$ \\
\hline Opportunities $(\mathrm{n}=51)$ & $\begin{array}{l}\text { Professional/Personnel Development \& } \\
\quad \text { Training } \\
\text { Resources/Funding } \\
\text { Miscellaneous } \\
\text { Communication/Collaboration } \\
\text { EHDI Programs/Services/Current Practices }\end{array}$ & $\begin{array}{r}18(35 \%) \\
15(29 \%) \\
7(14 \%) \\
6(12 \%) \\
5(10 \%)\end{array}$ \\
\hline Threats $(n=53)$ & $\begin{array}{l}\text { Funding } \\
\text { Miscellaneous/No Comments } \\
\text { Limited or Lack of Training Opportunities } \\
\text { Time } \\
\text { Lack of Providers } \\
\text { Compliance \& Regulations } \\
\text { Staffing }\end{array}$ & $\begin{array}{r}25(47 \%) \\
10(19 \%) \\
9(17 \%) \\
4(8 \%) \\
2(4 \%) \\
2(4 \%) \\
1(2 \%)\end{array}$ \\
\hline
\end{tabular}




\section{Strength Characteristics}

The three most frequently cited strengths were the range of professional development and training provided (58\%), the availability of funding to provide targeted training (14\%), and the ability to collaborate across agencies to provide training (10\%) (Table 2). Fifty-eight percent of EHDI coordinators reported that programs had successfully provided training covering a variety of topics (e.g., database training, pediatric audiology, early intervention, data tracking and referrals, multidisciplinary teaming, evidence-based practices) and targeting a range of disciplines (e.g., nurses, pediatric audiologists, early interventionists, physicians, administrators). Fourteen percent of respondents noted that funding is available to provide training on a limited basis and to utilize established outlets for continuing education, such as the annual national EHDI conference, state and local conferences and workshops, professional associations' statewide conferences, and coursework at local universities. Program funding, most commonly provided through federal agencies, was also used to support most training endeavors. Collaborative relationships between primary stakeholders and state agencies, universities, and professional associations was reported by $10 \%$ of respondents and allowed the pooling of resources to create professional development opportunities.

Other reported strengths included communication (7\%), such as newsletters, websites, and journal publications, and EHDI programs/services/

Table 2. Top three themes for strength

Themes

Range of Professional
Development Provided

Funding

Collaboration

\section{Descriptions}

Strong university affiliations to provide current education in all areas of public health.

The EHDI Program has planned \& implemented a biennial conference for hospitals \& audiologists. Speakers present three times each year on various topics.

Grant funding is available to send staff to the national EHDI conference.

State university system is currently developing a "health science system" that will focus on healthcare, including hearing loss.

Grants provided to allied professionals for development of professional trainings.

Regular meetings with stakeholders around the state on regional basis.

Close collaborative partnerships with organizations.

EHDI program partners with school districts to provide training to early interventionists \& preschool teachers. 
current practices (5\%), including the development of training goals, distribution of training materials, and implementation of an oversight program on hospital screeners.

\section{Weakness Characteristics}

The three most frequently cited weaknesses were lack of a perceived need for training $(18 \%)$, ongoing personnel shortages across disciplines $(15 \%)$, and programmatic barriers to training (12\%) (Table 3). Eighteen percent of respondents noted the attitudes of service providers, especially audiologists and early interventionists, toward professional development activities as a weakness. Low participation among service providers and reported attitudes demonstrating a "lack of interest or perceived need" for training contributed to the shortage of practitioners who were qualified to provide services. In addition to audiologists and early interventionists, shortages of qualified physicians, pediatric audiologists, nurses, and speech-language pathologists were also noted by $15 \%$ of EHDI coordinators. Barriers to training, as reported by $12 \%$ of the respondents, included a limited number of continuing education units/ credits offered for some professional development activities. The scheduling of continuing education opportunities often conflicted with other job responsibilities or appointments, and providing training opportunities to medical, allied health, and early intervention personnel in rural areas also proved challenging.

Other identified weaknesses included the need for or lack of professional development and training (10\%), funding ( $8 \%)$, lack of access to trainings

Table 3. Top three themes for weakness

\begin{tabular}{ll}
\hline \multicolumn{1}{c}{ Themes } & \multicolumn{1}{c}{ Descriptions } \\
\hline $\begin{array}{c}\text { Lack of Perceived } \\
\text { Need for Training }\end{array}$ & $\begin{array}{c}\text { Sometimes the providers that need the most training are } \\
\text { least likely to show up. } \\
\text { Limited attendance by medical professionals. } \\
\text { Many audiologists in the state do not want to provide } \\
\text { diagnostic testing of infants; therefore, they are not } \\
\text { interested in additional training. } \\
\text { Need more pediatric audiologists \& speech-language } \\
\text { pathologists. } \\
\text { Shortage of physicians \& nurses statewide. } \\
\text { Limited staff \& resources to provide the needed education. } \\
\text { The program staff members need training themselves and } \\
\text { are unable to obtain training at this time. } \\
\text { Access to various education credits for the various } \\
\text { provider types may be attending. } \\
\text { Rural state. }\end{array}$ \\
\hline
\end{tabular}


$(7 \%)$, lack of time for training $(7 \%)$, lack or resources $(7 \%)$, and limitations of travel to trainings (3\%).

\section{Opportunities for EHDI Programs}

The top three frequently cited opportunities were access to professional development and training (35\%), the availability of identified resources and funding to support professional development (29\%), and the ability to collaborate across agencies and service providers (12\%) (Table 4). Thirty-five percent of respondents reported opportunities for professional development and training were widespread, especially for audiologists. Partnerships with key stakeholders and state agencies provided a pooling of resources and greater access to consistent training and staff development. Three respondents mentioned the planned development of web-based training and the use of video conferencing equipment to facilitate greater access to and increased consistency of professional development opportunities. Twenty-nine percent of respondents noted that federal and state agencies, state professional associations/organizations, and local universities provided most of the funding used for professional development in EHDI programs. According to $12 \%$ of respondents, funding often required ongoing collaboration and cooperation with multiple federal and state agencies, universities, and key stakeholders.

Additionally, $10 \%$ of EHDI coordinators identified opportunities in current practices and services. Specifically they mentioned updating their state

Table 4. Top three themes for opportunity

\begin{tabular}{|c|c|}
\hline Themes & Descriptions \\
\hline $\begin{array}{l}\text { Access to Professional } \\
\text { Development \& Training }\end{array}$ & $\begin{array}{l}\text { Good opportunities for pediatric audiology training. } \\
\text { Work with other state agencies \& private entities to } \\
\text { offer conferences \& workshops. } \\
\text { Early intervention taskforce is working on providing } \\
\text { training for a streamlined developmental checklist. }\end{array}$ \\
\hline $\begin{array}{l}\text { Availability of Identified } \\
\text { Resources \& Funding }\end{array}$ & $\begin{array}{l}\text { Able to collaborate with other agencies in some efforts. } \\
\text { Online conference technology has been purchased, } \\
\text { which can be used for staff-to-staff training. } \\
\text { Rich academic area. }\end{array}$ \\
\hline $\begin{array}{l}\text { Ability to Collaborate Across } \\
\text { Agencies }\end{array}$ & $\begin{array}{l}\text { Establishing a state workgroup to begin focusing on } \\
\text { improving the components of the EHDI } \\
\text { system. } \\
\text { Able to collaborate with other agencies in some efforts. } \\
\text { Working with residency programs in hospitals \& linking } \\
\text { family support closely with early intervention. }\end{array}$ \\
\hline
\end{tabular}


guidelines to reflect best practices, creating educational sub-committees, and participation in NICHQ's Learning Collaborative project.

\section{Threats to EHDI Programs}

The three most frequently cited threats were inadequate funding and resources $(47 \%)$, a limited or lack of perceived need for training $(17 \%)$, and time limitations for training (8\%) (Table 5). Forty-seven percent of EHDI programs reported significant challenges in supporting ongoing professional development due to inadequate funding and resources. Because of local and state budget issues and other economic factors, funding for professional development initiatives had been sharply reduced. Likewise, travel funds have been cut or eliminated entirely, which also limits the number of training opportunities available. When training opportunities were offered, $17 \%$ of respondents reported that smaller numbers of the targeted professionals would engage in training. That is, many professionals felt they no longer needed additional training to provide services to children with hearing loss and their families. And finally, $17 \%$ of respondents noted that travel restrictions, work schedule restrictions, and a dearth of training opportunities in specific areas of a state prevented professionals from receiving ongoing training.

Other threats identified by EHDI coordinators include a lack of audiology providers $(4 \%)$, compliance by professionals to follow EHDI guidelines $(4 \%)$, and lack of staff to provide necessary trainings $(2 \%)$.

Table 5. Top three themes for threat

\begin{tabular}{|c|c|}
\hline Themes & Descriptions \\
\hline $\begin{array}{l}\text { Inadequate Funding \& } \\
\text { Resources }\end{array}$ & $\begin{array}{l}\text { Lack of funding. } \\
\text { Need continued funding to keep training opportunities. } \\
\text { Potential funding sources disappearing that are currently } \\
\text { used to keep EHDI personnel current with information. }\end{array}$ \\
\hline $\begin{array}{l}\text { Lack of Perceived Need } \\
\text { for Training }\end{array}$ & $\begin{array}{l}\text { Education/professional development interests of many } \\
\text { audiologists \& administrators focus more on older } \\
\text { children \& adult populations. } \\
\text { Lack of interest (audiologists can make more money } \\
\text { treating adults). } \\
\text { No "teeth" to implementing audiological guidelines or } \\
\text { JCIH } 2007 \text { position statement. }\end{array}$ \\
\hline $\begin{array}{l}\text { Barriers to Travel, Time } \\
\text { Limitations, \& } \\
\text { Restricted Opportunities } \\
\text { for Training }\end{array}$ & $\begin{array}{l}\text { Current travel restrictions allow no travel for training } \\
\text { unless it is } 100 \% \text { paid by non-state funds. } \\
\text { Hard for staff to leave their practice to attend training \& } \\
\text { other professional development opportunities. } \\
\text { Very limited opportunities for in-state professional } \\
\text { development. }\end{array}$ \\
\hline
\end{tabular}




\section{TOWS Analysis Matrix}

By applying the TOWS analysis matrix to the themes identified in the SWOT analysis, specific recommendations, or "strategic options," were derived from the internal and external environmental factors identified by the EHDI coordinators. By systematically pairing the four categories of the SWOT analysis, recommendations can be derived to maximize resources and effect positive change. Based on the SWOT themes, 12 individual recommendations emerged (Table 6). To maximize opportunities, identification of strengths can lead to positive change (S-O strategy). For example, by working with university and online training programs, there are more opportunities to provide training to existing providers in newborn hearing screening, evaluation, and follow-up care areas. By pursuing opportunities, weaknesses can be minimized (W-O strategy). For example, advertising training opportunities to a targeted audience may foster greater participation. Identification of ways to use strengths can ultimately reduce vulnerability to threats (S-T strategy). For example, pursuing collaborative relationships between agencies and professional entities may reduce the impact on resources, eliminate barriers to travel, and increase availability of training opportunities. Finally, establishing defensive plans will help to prevent weaknesses from making programs susceptible to threats (W-T strategy). For example, developing program policies and procedures and clearly defining expectations can eliminate barriers to trainings. From the 12 recommendations, 3 distinct strategies emerged for improving professional development opportunities. Specifically, state EHDI programs should: 1) target personnel shortages by providing a range of professional development activities for medical, clinical, and educational professionals who deliver direct services to young children with hearing loss and their families; 2) establish policies and procedures to eliminate barriers to training; and 3) publicize all training opportunities to foster greater participation.

\section{Limitations}

Limitations of this study include those inherent in the application of a SWOT/TOWS analysis when applied to rapidly changing conditions. SWOT analysis is a subjective tool that explores conditions that are perceived rather than objectively measured. In this sense, the SWOT analysis is influenced by the perspectives of those who completed the surveys as well as those who analyzed the responses. Furthermore, SWOT analysis characterizes the situation at a specific point in time. In addition, SWOT analysis, as applied here, resulted in overlapping themes within a category. For example, funding and professional development training opportunities could be identified as a strength, weakness, opportunity, and threat (see White \& Blaiser, 2011, for additional information regarding the selection of SWOT/TOWS for this study and further comment regarding interpretation and application of findings). 
Table 6. TOWS analysis matrix for professional development

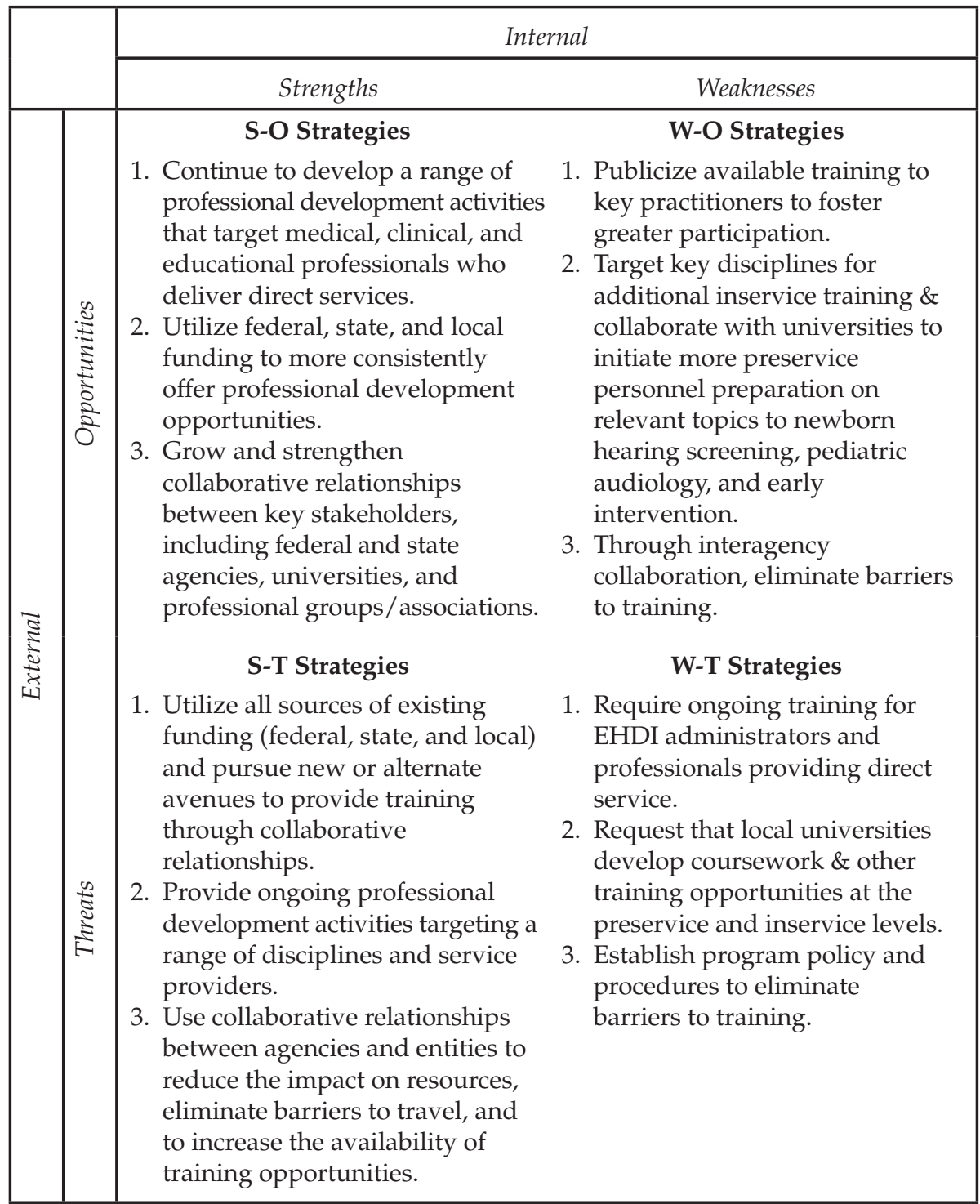

$\mathrm{S}=$ Strength $\mathrm{W}=$ Weakness $\mathrm{O}=$ Opportunity $\mathrm{T}=$ Threat

\section{Summary}

Three recurring themes related to professional development emerged in this analysis: 1) the range of professional development activities offered, 2) funding, and 3) collaboration. That is, EHDI programs are collaborating with other agencies, professional associations, and universities to provide 
professional development and training; however, critical shortages of wellqualified practitioners (e.g., physicians, nurses, pediatric audiologists, speechlanguage pathologists, teachers of the deaf, administrators) remain. There is limited funding through federal, state, and local agencies to provide professional development activities, and funding shortages are impacting the frequency and consistency of professional development in most EHDI programs. Long-term opportunities for continued training may require pooling resources and building cooperative relationships with other professional groups, organizations, and state agencies. Some barriers may be eliminated through increased funding, innovative practices for training (e.g., web/video conferencing and distance learning), expansion of stakeholder partnerships, and the establishment of required continuing education for administrators and direct service providers.

Through this study, EHDI coordinators have described the availability of professional development activities in most states. However, because of budget constraints, the frequency of training opportunities remains in jeopardy. By establishing partnerships and cooperative relationships, key stakeholders are finding ways to provide some training; however, considerable shortages remain in the number of qualified practitioners who can provide direct services to very young children with hearing loss, resulting in families who are often underserved. Continued planning, partnering, and funding are needed to ensure that more educational opportunities are available at both the preservice and inservice levels. Only then will the threat of inadequate service delivery due to personnel shortages be successfully ameliorated.

\section{Acknowledgement}

The work reported in this article was funded in part by the Maternal and Child Health Bureau under Cooperative Agreement \# U52MC04391 with the National Center for Hearing Assessment and Management at Utah State University. The opinions expressed in the article are those of the authors and do not necessarily reflect those of the Bureau.

\section{References}

American Academy of Pediatrics Medical Home Initiatives for Children with Special Needs Project Advisory Committee. (2002). The medical home. Pediatrics, 110(1), 184-186.

American Speech-Language-Hearing Association (ASHA). (2004). Roles of speech-language pathologists and teachers of children who are deaf and hard of hearing in the development of communicative and linguistic competence. Retrieved May 19, 2011, from http:/ /www.asha.org/docs/html/TR2004-00256.html. American Speech-Language-Hearing Association (ASHA). (2008). Service provision to children who are deaf and hard of hearing, birth to 36 months. Retrieved May 19, 2011, from http:/ /www.asha.org/docs/html/TR2008-00301.html. 
Apuzzo, M. L., \& Yoshinaga-Itano, C. (1995). Early identification of infants with significant hearing loss and the Minnesota child development inventory. Seminars in Hearing, 16, 124-137.

Calderon, R. (2000). Parental involvement in deaf children's education programs as a predictor of child's language, early reading, and social-emotional development. Journal of Deaf Studies and Deaf Education, 5, 140-155.

Compton, M. V., Tucker, D. A., \& Flynn, P. F. (2009). Preparation and perception of speech-language pathologists working with children with cochlear implants. Communication Disorders Quarterly, 30(3), 142-154.

Houston, K. T., \& Caraway, T. (2010). For children with hearing loss: The times, they are a-changin. Perspectives on Hearing and Hearing Disorders in Childhood, 20(2), 48-55.

Houston, K. T., \& Perigoe, C. B. (2010a). Speech-language pathologists: Vital listening and spoken language professionals. The Volta Review, 110(2), 219-230.

Houston, K. T., \& Perigoe, C. B. (2010b). Future directions in professional preparation and development. The Volta Review, 110(2), 339-340.

Johnson, H. A. (2004). U.S. deaf education teacher preparation programs: A look at the present and a vision for the future. American Annals of the Deaf, 149(2), 75-91.

Joint Committee on Infant Hearing (JCIH). (2007). Year 2007 position statement: Principles and guidelines for early hearing detection and intervention programs. Pediatrics, 120(4), 898-921.

Lenihan, S. (2010). Trends and challenges in teacher preparation in deaf education. The Volta Review, 110(2), 117-128.

Luckhurst, J. A. (2008). Professional preparedness for provision of auditory oral programs to children with hearing loss: Results of a survey. Perspectives on Aural Rehabilitation and Its Instrumentation, 15, 2-18.

Mayne, A. M., Yoshinaga-Itano, C., \& Sedey, A. L. (1998). Receptive vocabulary development of infants and toddlers who are deaf or hard of hearing. The Volta Review, 100(5), 29-52.

Moeller, M. P. (2000). Early intervention and language development who are deaf and hard of hearing. Pediatrics, 106(3), E43.

Moeller, M. P., White, K. R., \& Shisler, L. (2006). Primary care physicians' knowledge, attitudes, and practices related to newborn hearing screening. Pediatrics, 118(4), 1357-1370.

Pipp-Siegel, S., Sedey, A. L., VanLeeuwen, A. M., \& Yoshinaga-Itano, C. (2003). Mastery motivation and expressive language in young children with hearing loss. Journal of Deaf Studies and Deaf Education, 8, 133-145.

Robbins, A. M. \& Caraway, T. (2010). Missing the mark in early intervention for babies who are hard of hearing or deaf learning spoken language. Perspectives on Hearing and Hearing Disorders in Childhood, 20(2), 41-47.

Roush, J. (2010). Educational preparation of pediatric audiologists. The Volta Review, 110(2), 207-218. 
Shulman, S., Besculides, M., Saltzman, A., Ireys, H., \& White, K. R. (2010). Evaluation of the universal newborn hearing screening and intervention program. Pediatrics, 126, S19-S27.

Vernon, J. (2007). Preparing professionals for a changing landscape. Volta Voices, 14(6), 18-21.

White, K. R. (2007). Early intervention for children with permanent hearing loss: Finishing the EHDI revolution. The Volta Review, 106(3), 237-258.

White, K. R. (2008). Newborn hearing screening. In J. R. Madell and C. Flexer (Eds.), Pediatric audiology: Diagnosis, technology, and management (pp. 31-41). New York: Thieme.

White, K., \& Blaiser, K. (2011). Strategic planning to improve early hearing detection and intervention programs. The Volta Review, 111(2), 83-108.

White, K. R., Forsman, I., Eichwald, J., \& Munoz, K. (2010). The evolution of early hearing detection and intervention programs in the United States. Seminars in Perinatology, 31(2), 170-189.

Yoshinaga-Itano, C. (2001). The social-emotional ramifications of universal newborn hearing screening: Early identification and intervention of children who are deaf or hard of hearing. In Proceedings of the Second International Pediatric Conference: A Sound Foundation Through Early Amplification. Stafa, Switzerland: Phonak.

Yoshinaga-Itano, C., Sedey, A. L., Coulter, D. K., \& Mehl, A. L. (1998). Language of early- and later-identified children with hearing loss. Pediatrics, 102, $1161-1171$. 

The Volta Review, Volume 111(2), Summer 2011, 225-242

\title{
Is the Infrastructure of EHDI Programs Working?
}

\author{
K. Todd Houston, Ph.D., CCC-SLP, LSLS Cert. AVT; \\ Jeff Hoffman, M.S., CCC-A; Karen F. Muñoz, Ed.D., CCC-A; and \\ Tamala S. Bradham, Ph.D., CCC-A
}

State coordinators of early hearing detection and intervention (EHDI) programs completed a strengths, weaknesses, opportunities, and threats, or SWOT, analysis that consisted of 12 evaluative areas of EHDI programs. For the EHDI program infrastructure area, 47 coordinators responded with a total of 292 items, and themes were identified in each category. A threats, opportunities, weaknesses, and strengths, or TOWS, analysis yielded 12 recommendations that focused on similar themes. Out of these 12 recommendations, 3 distinct strategies emerged: 1) continue utilizing the EHDI advisory committee to establish and expand collaborative agreements with key stakeholders; 2) maximize funding opportunities from state and local sources; and 3) raise public awareness about the purpose and importance of newborn hearing screening, early diagnosis, and early intervention for children with hearing loss. Addressed appropriately, these recommendations can strengthen the infrastructure of state EHDI programs.

\section{Introduction}

An effective and efficient early hearing detection and intervention (EHDI) program depends on a strong infrastructure consisting of several key components. For example, Leigh, Schmulian-Taljaard, and Poulakis (2010) describe three components that are common to all newborn hearing screening programs: 1) an initial screen, 2) audiological and medical evaluation to ascertain

\footnotetext{
K. Todd Houston, Ph.D., CCC-SLP, LSLS Cert. AVT, was an Assistant Professor in the Department of Communicative Disorders and Deaf Education at Utah State University at the time of this study and is currently at The University of Akron. Jeff Hoffman, M.S., CCC-A, is a Senior Research Scientist - Audiologist in the National Center for Hearing Assessment and Management at Utah State University. Karen F. Muñoz, Ed.D., CCC-A, is an Assistant Professor in the Department of Communicative Disorders and Deaf Education at Utah State University. Tamala S. Bradham, Ph.D., CCC-A, is an Assistant Professor in the School of Medicine, Department of Hearing and Speech Sciences, at Vanderbilt University. Correspondence concerning this manuscript should be addressed to Dr. Houston at ktodd.houston@gmail.com.
} 
the presence, type, and severity of the condition (i.e., hearing loss), and 3) (re)habilitation, treatment, or therapy for those children who are found to have hearing loss. Today, all states conduct newborn hearing screening, resulting in more than $97 \%$ of newborn infants in the United States being screened prior to discharge from the hospital or birthing center (Centers for Disease Control and Prevention [CDC], 2010a). While achieving this level of hearing screening represents a significant milestone, almost half of the infants referred from newborn screening do not receive appropriate and timely follow-up to confirm the presence of hearing loss and/or initiate early intervention services (Joint Committee on Infant Hearing [JCIH], 2007). Thus, it is reasonable to wonder how well the infrastructure of state EHDI programs is working.

State EHDI programs have made significant strides in establishing infrastructures and systems that support the initial screening, follow-up audiological and medical diagnostics, and referral for early intervention and (re)habilitative services. In fact, 43 states now have newborn hearing screening mandated as part of state statute, policy, or public law (White, Forsman, Eichwald, \& Muñoz, 2010a). However, rates of screening, diagnosis, and follow-up vary from state-to-state due to several factors, such as staffing, funding, legislative, and/or regulatory actions, the role and function of the state EHDI advisory committee, and public awareness and support of EHDI program services. These system-level components are likely to have a substantial impact on a state EHDI program's overall effectiveness.

\section{Staffing EHDI Programs}

In a recent survey, Allen and Houston (2010) found that EHDI programs are typically housed within a state's department of health and human services and often have at least one full-time staff member. Occasionally, the EHDI program may be located in a state department of education. While some exceptions exist, state-level coordination of most EHDI programs is usually provided by an audiologist or a professional from a related field.

The role of the state EHDI coordinator is critical and coordinators must be able to transform independent private and public agencies into a cohesive and functional team. McCollister (2010) describes the tasks and skill sets needed by a state EHDI coordinator, which include knowledge of grant writing and management of funding through federal, state, and local funding agencies as well as accountability, efficiency, and teamwork ability. Unfortunately, annual turnover in these positions has been at $20-25 \%$ in recent years (K. R. White, National Center for Hearing Assessment \& Management [NCHAM], personal communication, September 30, 2010). With nearly one-fourth of state EHDI coordinators resigning each year, breakdowns in program coordination and infrastructure are inevitable. 
Effective hospital-based newborn hearing screening programs require a high-level of program coordination and a commitment to ongoing quality improvement and evaluation (Leigh, et al., 2010; White, 2008). Staffing screening programs remains a challenge due to the array of nurses, audiologists, hospital staff, and volunteers who actually conduct the screening. Shulman, Besculides, Saltzman, Ireys, and White (2010) noted that most hospital-based hearing screening is performed by nursing and newborn care staff, and although some newborn hearing screening programs provide initial protocols or training materials, most screeners were trained by other screeners or by equipment manufactures without guidance from the hospital-based screening program. Staff turnover among hospital screeners remains high, which has necessitated improved training materials and procedures, well-defined screening protocols, and increased efforts to recruit and retain qualified screeners (White, 2008; Winston, 2008).

Audiologists, by the nature of their educational preparation, may serve a variety of roles within a screening program, including newborn screening, program development, management, quality assessment, service coordination, referral for audiological diagnosis, and treatment (JCIH, 2007). For infants referred from a hearing screen, the audiologist provides comprehensive diagnostic assessment to confirm a hearing loss, to ensure that parents understand the implications of the hearing loss, to evaluate the infant's candidacy for amplification and other sensory devices chosen by the family, and to ensure prompt referral to early intervention programs (JCIH, 2007). The role of the pediatric audiologist is vital to the success to state EHDI programs. Unfortunately, there is a shortage of pediatric audologists with the expertise needed to diagnosis hearing loss in infants and provide them with appropriate amplification (Nemes, 2006; Shulman, et al., 2010; White, 2003). Sieminski (2010) notes that successful state EHDI programs require a seamless system that incorporates hearing screening, prompt diagnosis, effective intervention, and statewide tracking of infants identified with hearing loss. Unfortunately, Sieminski also observes, "Most hospital EHDI programs have little or no involvement with an audiologist. Many hospitals do not have an audiologist to ensure effective screening, early diagnosis, and appropriate referrals. There are too few pediatric audiologists in many rural areas to provide easy access and early detection and intervention" (2010, p. 35).

For the infant who does not pass a newborn hearing screening, medical evaluation is required prior to the diagnostic audiology assessment. Typically, the physician who examines the infant is either a pediatrician or a family physician. Shulman et al. (2010) found that many pediatricians have significant gaps in their knowledge of childhood hearing loss because it is often not included in their medical training. Similarly, Moeller, White, and Shisler (2006) found knowledge deficits related to newborn hearing screening, diagnosis, use of hearing technology, and early intervention among pediatricians, 
family physicians, and other medical specialties. These are serious concerns considering that after a diagnosis of hearing loss is confirmed, prompt referral to early intervention is essential. Yet, many state EHDI coordinators report that lack of knowledge by pediatricians and audiologists about the availability of early intervention services is a major problem in their states (Shulman, et al., 2010).

Numerous studies underscore the developmental, communicative, and social benefits of early intervention for young children identified with hearing loss (Apuzzo \& Yoshinaga-Itano, 1995; Calderon, 2000; Mayne, Yoshinaga-Itano, \& Sedey, 1998; Moeller, 2000; Pipp-Siegel, Sedey, VanLeeuwen, \& YoshinagaItano, 2003; Yoshinaga-Itano, 2001; Yoshinaga-Itano, Sedey, Coulter, \& Mehl, 1998). Even so, early intervention services available to infants and toddlers with hearing loss are insufficient due to a lack of service providers (e.g., speech-language pathologists, teachers of the deaf) with the knowledge and skills needed to serve this population (Compton, Tucker, \& Flynn, 2009; Houston \& Caraway, 2010; Houston \& Perigoe, 2010; Johnson, 2004; Lenihan, 2010; Luckhurst, 2008; Robbins \& Caraway, 2010; Vernon, 2007). Some investigators attribute the shortage of qualified service providers to the fact that most early intervention programs were designed to serve children who were identified at 2 to 3 years of age (White, Forsman, Eichwald, \& Muñoz, 2010b); these same state EHDI programs are insufficiently staffed to accommodate the increased number of infants and toddlers who are now being identified through newborn hearing screening programs (Shulman, et al., 2010; White, 2007). Thus, the capacity to serve increasing numbers of young children with hearing loss within state EHDI programs remains limited, and state EHDI coordinators describe the lack of appropriate early intervention services as a major problem (Shulman, et al., 2010).

\section{Funding and Sustainability}

Since the 1960s, federal reports, consensus panels, commissions, and working groups have studied the feasibility and development of systematic universal newborn hearing screening and tracking programs (See White, et al., 2010a, for a detailed discussion). Likewise, professional organizations have joined these efforts, including the $\mathrm{JCIH}$, which has taken a lead role in offering guidance to state EHDI programs. Because of the evolution of funding and policy initiatives at the federal level, the Maternal and Child Health Bureau $(\mathrm{MCHB})$ and the $\mathrm{CDC}$ have also taken a shared role in supporting state EHDI programs.

As with any public or private endeavor of this nature, continuous and reliable funding streams are critical for long-term viability and sustainability. According the 2004 EHDI State Survey (Faust \& Ditty, 2010; NCHAM, 2004), the average state EHDI program budget was $\$ 538,573$, with a range of $\$ 77,200$ to $\$ 3,460,000$. However, state EHDI programs indicate that an average of 
$69 \%$ of their funding is received from either the CDC or MCHB (NCHAM, 2004). With dependence on federal funding, any reduction in this support may severely jeopardize the effectiveness of most, if not all, state EHDI programs.

The level of funding available to state EHDI programs varies considerably. While 43 states (plus the District of Columbia and Puerto Rico) have legislative statutes related to EHDI (NCHAM, 2010), these statutes and policies vary according to specificity of services, reporting requirements, personnel, and funding allocations. During challenging economic periods, state legislatures and administrators may attempt to reduce funding to state EHDI programs. As Faust and Ditty explain, "the financial burden of the EHDI system must be balanced against the significant savings in special education and increased productivity of those who are permitted to reach their full potential. The concept of spending money now to save it later may not register with legislators faced with tight budgets or third-party payers concerned with the bottom line. EHDI programs must make every effort to remain financially viable and continue to be the lifeline for families of deaf and hard of hearing children" (2010, p. 15-8).

\section{Legislative and Regulatory Action}

Although a clear majority of states (43) have legislation related to early diagnosis, treatment, and intervention for children with hearing loss, there is considerable variability in legislation and public policies among states. For example, White et al. (2010a) reported that only 28 of the 43 states with legislative mandates $(65 \%)$ require screening of all infants; others require only $85 \%$ of newborns to be screened. Green, Gaffney, Devine, and Grosse (2007) examined screening rates from $2000-2003$ and found that $76 \%$ of the states that had implemented newborn hearing screening legislation reported screening at least $95 \%$ of infants, compared with $26 \%$ of states without legislation. Green et al. (2007) also found that legislative mandates had a positive effect on hearing screening rates and were an important tool in assuring that infants receive appropriate screening for hearing loss. As Faust and Ditty advise, "It is important to note that legislation outlines the minimum expectations of state policy-makers but does not necessarily define all of the activities that state EHDI programs are doing...If enacting a legislative mandate or strengthening an existing mandate is not feasible or politically prudent, programs can still be strengthened and sustained through strong coalitions, partnerships, and agreements" (2010, p. 10-12).

\section{The Role of the Advisory Committee}

The CDC has developed national goals that define preferred practices and performance indicators for state EHDI programs (CDC, 2010b). One such goal 
clearly outlines the need for a system to monitor and evaluate progress toward achieving national EHDI goals. Critical to this system of monitoring and evaluation is the role of the advisory committee. As White et al. (2010a) state, "an EHDI advisory committee in each state should take the responsibility for developing and maintaining such a system" (2010a, p. 1-13). Almost all states currently have a state EHDI advisory committee that meets regularly at least quarterly. While membership varies among state EHDI advisory committees, most committees have representation from key stakeholders, such as audiologists, early intervention providers (e.g., teachers of the deaf, speechlanguage pathologists, early childhood educators), primary health care providers, program and/or agency administrators, representatives from the deaf community, and parents of children with hearing loss. According to the 2004 state EHDI survey (NCHAM, 2004; White, et al., 2010a), states reported a variety of methods for gathering gather information used for evaluation and monitoring. Specifically, 31 states used site visits, 36 reviewed archival data, 20 did surveys or focus groups with hospitals, 15 conducted surveys or focus groups with parents, and 7 completed surveys with primary care physicians. Unfortunately, only 18 states reported completing a systematic evaluation of the state's EHDI program within the past 5 years. As White et al. (2010a) notes, "These data suggest that it would be valuable for states to devote more attention to evaluation and monitoring of EHDI programs" (2010a, p. 1-13).

\section{Public Awareness and Support}

The importance of newborn hearing screening has been supported and promoted by an array of federal agencies (e.g., CDC, MCHB), professional organizations (e.g., American Speech-Language-Hearing Association, American Academy of Pediatrics), and advocacy and family support organizations (e.g., Alexander Graham Bell Association for the Deaf and Hard of Hearing, Hands \& Voices). These government agencies, professional groups, and nonprofit organizations often have collaborated on national and state levels to support legislation and various public awareness efforts to emphasize the importance of early identification of hearing loss, treatment, and intervention.

At the state level, most EHDI advisory committees have representatives from the above mentioned stakeholder groups. For the state EHDI program coordinator, the advisory committee, with its varied stakeholder representation, can be an effective device for expanding public awareness and support. Similarly, McCollister (2010) notes that the state EHDI coordinator should take every opportunity to communicate the importance of typical development of speech and language through early identification and intervention. McCollister suggests several possible strategies, including sharing information about the EHDI program, available services, and important 
benchmarks for children who are deaf and hard of hearing; providing human interest stories about successful children through multiple media outlets (e.g., newspapers, television, radio, etc.); conducting media interviews and providing public service announcements; serving on interagency taskforces, advisory boards, and training programs; and interacting with professionals who provide direct services to children who are deaf and hard of hearing.

This study was undertaken to examine the current status of key elements that affect the infrastructure of state EHDI programs, including staffing, funding, legislative, and regulatory actions, the function of the EHDI advisory committee, and public awareness and support. This was accomplished through a systematic review of EHDI program infrastrcuture using a strengths, weaknesses, opportunities, and threats, or SWOT, and a threats, opportunities, weaknesses, and strengths, or TOWS, analysis to determine the current status of EHDI program infrastructures and to identify factors to improve program effectiveness and outcomes for infants with hearing loss.

\section{Methods}

Fifty-one EHDI coordinators were asked to complete an online survey. Related to program infrastructure, the survey asked for the EHDI coordinator to report at least 1 item in the following four areas to assist with strategic planning: strength, weakness, opportunity, and threat. The responses obtained were reviewed by a panel of experts in the field and categorized into common themes in each of these four strategic planning areas. To generate recommendations from the SWOT analysis, a TOWS matrix was used to match identified strengths with opportunities (S-O strategy), strengths with threats (S-T strategy), weaknesses with opportunities (W-O strategy), and weaknesses with threats (W-T strategy). For an in-depth review of the methodology, the reader is referred to White and Blaiser, 2011.

\section{Results}

Forty-seven EHDI coordinators (92\%) completed the infrastructure area of the SWOT questionnaire. The respondents generated 100 items in the strengths category, 73 items in the weaknesses category, 64 items in the opportunities category, and 55 items in the threats category for a total of 292 responses. Based on these responses, several specific themes were generated for each category: 10 for strengths, 9 for weaknesses, 9 for opportunities, and 5 for threats (Table 1). Each category also had a miscellaneous section that included no comment, N/A, or items that did not fit within the themes identified. 
Table I. Frequency of themes within each SWOT category $(n=292)$

\begin{tabular}{|c|c|c|}
\hline SWOT category & Themes & Frequency n (\%) \\
\hline Strengths $(n=100)$ & $\begin{array}{l}\text { Legislation/Regulations } \\
\text { Effective and Engaged Advisory } \\
\quad \text { Committee } \\
\text { Staffing } \\
\text { Funding } \\
\text { Commitment } \\
\text { Miscellaneous/No comment } \\
\text { Data System } \\
\text { System Support } \\
\text { Partnerships } \\
\text { Organizational Structure }\end{array}$ & $\begin{array}{l}17(17 \%) \\
17(17 \%) \\
15(15 \%) \\
12(12 \%) \\
11(11 \%) \\
7(7 \%) \\
6(6 \%) \\
6(6 \%) \\
6(6 \%) \\
3(3 \%)\end{array}$ \\
\hline Weaknesses $(n=73)$ & $\begin{array}{l}\text { Funding } \\
\text { Staffing } \\
\text { Public Awareness } \\
\text { Legislation/Regulations } \\
\text { Miscellaneous/No Comment } \\
\text { Advisory Committee } \\
\text { Commitment } \\
\text { Geography } \\
\text { Data System }\end{array}$ & $\begin{array}{c}19(26 \%) \\
19(26 \%) \\
10(14 \%) \\
7(10 \%) \\
6(8 \%) \\
5(7 \%) \\
3(4 \%) \\
2(3 \%) \\
2(3 \%)\end{array}$ \\
\hline Opportunities $(n=64)$ & $\begin{array}{l}\text { Miscellaneous/No Comments } \\
\text { Partnerships } \\
\text { Data Systems } \\
\text { Funding } \\
\text { Staffing } \\
\text { System Support } \\
\text { Public Awareness } \\
\text { Legislation } \\
\text { Advisory Committee }\end{array}$ & $\begin{array}{l}11(17 \%) \\
9(14 \%) \\
8(13 \%) \\
8(13 \%) \\
8(13 \%) \\
7(11 \%) \\
6(9 \%) \\
5(8 \%) \\
2(3 \%)\end{array}$ \\
\hline Threats $(n=55)$ & $\begin{array}{l}\text { Funding } \\
\text { Legislation/Regulations } \\
\text { Miscellaneous/No Comments } \\
\text { Staffing } \\
\text { Public Awareness }\end{array}$ & $\begin{array}{c}34(62 \%) \\
7(13 \%) \\
7(13 \%) \\
5(9 \%) \\
2(4 \%)\end{array}$ \\
\hline
\end{tabular}

\section{Strength Characteristics}

The most frequently cited strengths were the role of the advisory committee and legislative and regulatory actions (tied, $17 \%)$, quality of staff $(15 \%)$, and the availability of funding (12\%) (Table 2). Seventeen percent of state EHDI coordinators reported an active and supportive advisory committee with representation from a wide variety of stakeholders and constituents. Advisory committees have been successful at advocating for issues related to newborn 
Table 2. Top four themes for strengths

\begin{tabular}{cl}
\hline \multicolumn{1}{c}{ Themes } & \multicolumn{1}{c}{ Descriptions } \\
\hline Legislation/Regulations & $\begin{array}{c}\text { Recently passed state legislation supporting the EHDI } \\
\text { program. } \\
\text { State regulations require hearing screenings in hospitals. } \\
\text { Very active, engaged, and supportive advisory } \\
\text { committee. }\end{array}$ \\
Committee & $\begin{array}{l}\text { Staffing in place at the state level. } \\
\text { Consistent staff for 4-5 years. }\end{array}$ \\
& Currently fully staffed. \\
Funding & Grant funding in place. \\
& State legislature recently provided funding. \\
& Federal funding (HSRA \& CDC). \\
\hline
\end{tabular}

hearing screening, diagnosis of hearing loss, and early intervention. Legislative and regulatory action was tied (with role of the advisory committee) among EHDI coordinators for top strengths. Examples provided by EHDI coordinators included strong mandates or legislation passed by their states to support EDHI programs. The third most common strength theme was quality of staff, with $15 \%$ of coordinators reporting increased staff support and positions, knowledgeable and consistent staff, and the addition of audiologists in key positions. The final top strength theme was availability of funding. Twelve percent of EHDI coordinators reported that federal funding for surveillance and follow-up activities helped build infrastructure. Others reported a balance of federal (e.g., Health Resources and Services Administration, CDC) and state funding that provided resources for staffing (salaries), screening equipment for hospitals, birthing centers, midwives, and promotional activities.

EHDI coordinators also reported commitment (11\%), such as active and committed stakeholders, staff, hospitals, and patients; data systems (6\%), such as web-based data management systems to collect data from hospitals and community providers; system support $(6 \%)$, such as having other state EHDI programs, NCHAM, advisory boards, and committees to call upon for support; partnerships (6\%); and organizational structure $(3 \%)$, such as where the EHDI programs was housed within the governmental structure, as strengths.

\section{Weakness Characteristics}

The most frequently cited weaknesses were staffing and funding (tied, 26\%), public awareness and support (14\%), and legislative and regulatory actions $(10 \%)$ (Table 3). A total of $52 \%$ of the responses within the weakness category focused on staffing and funding needs, with both themes receiving $26 \%$ of the responses, respectively. Coordinators reported a shortage of key personnel 
Table 3. Top four themes for weaknesses

\begin{tabular}{|c|c|}
\hline Themes & Descriptions \\
\hline Staffing & $\begin{array}{l}\text { Lack of state staff for program oversight. } \\
\text { Lack of staff; lost staff due to budget cuts. }\end{array}$ \\
\hline Funding & No state funding; state budget crisis. \\
\hline Public Awareness & $\begin{array}{l}\text { Lack of public awareness about EHDI. } \\
\text { Lack of awareness within state government. } \\
\text { Public awareness of hearing screening could be higher. }\end{array}$ \\
\hline $\begin{array}{l}\text { Legislative \& Regulatory } \\
\text { Actions }\end{array}$ & $\begin{array}{l}\text { EHDI program is unfunded in the state statute. } \\
\text { EHDI program is not mandated. } \\
\text { Legislative changes take time and support. }\end{array}$ \\
\hline
\end{tabular}

needed to support state EHDI systems, especially administrative program staff. Some coordinators reported being understaffed administratively and that key positions at the state level had been reduced by funding cuts. Regarding funding, some coordinators report that their state EHDI programs are primarily funded by federal grants, with little or no funding from the state. Coordinators expressed considerable concern with regard to long term viability of state EHDI programs if federal funding is reduced or eliminated.

Public awareness and support were reported as weaknesses by $14 \%$ of respondents. Coordinators expressed concern regarding lack of public awareness about the need for hearing screening and EHDI programs. They also noted lack of awareness among state legislators, administrators of other state departments and programs, and other key stakeholders (e.g., audiologists, physicians, early interventionists).

Legislative and regulatory actions comprised 9\% of the weaknesses noted. Some coordinators reported that their state did not have a mandate that supported the EHDI program or insurance coverage for hearing aids and care coordination. Other coordinators noted that the legislative process within their state takes considerable time and support to either get legislation passed and/or to modify existing mandates and policies. In some states, where a mandate to support the EHDI program was in place, funding for the EHDI program was not specified or defined in the legislation.

EHDI coordinators also reported the following weaknesses in their infrastructure: advisory committee concerns (7\%), commitment $(4 \%)$, geography $(3 \%)$, and data systems $(3 \%)$. Issues surrounding the advisory committee included decreased size, members work full time (and therefore it is difficult to engage them), lack of participation, or no advisory board or committees. Related to commitment, lack of trust among stakeholders, no unified agenda, and lack of involvement by the medical community were specifically noted. The geographical size of the state was also reported as a difficult challenge. 
The most frequently cited opportunities were partnerships among stakeholders $(14 \%)$, data systems, funding, and staffing (tied at $13 \%$ each), and system support (11\%) (Table 4). Fourteen percent of states reported that increased collaboration among key stakeholders was a major opportunity for the EHDI program. Coordinators reported improved cooperation with staff within the state's Newborn Metabolic Screening program, members of the Deaf community, and the March of Dimes. Coordinators also expressed the need to foster collaboration among stakeholder groups as a means of improving service coordination, communication, data collection, and surveillance.

Data systems, funding, and staffing (tied) comprised a combined total of $38 \%$ of the coordinators' responses. EHDI coordinators reported improved data systems used to track newborns who do not pass newborn hearing screening as an opportunity, and some states reported enhanced databases due to greater integration with newborn metabolic screening and online birth certificate systems. Coordinators also reported that funding to support the EHDI program had been identified through metabolic screening within the state, which allowed for a more stable funding stream. Other coordinators reported limited funding, typically provided through federal sources, to obtain equipment for midwives to conduct screenings, funds to attend conferences for training, and funds to upgrade database software. Finally, state coordinators reported plans to either hire or contract with new staff members to meet

Table 4. Top five themes for opportunities

\begin{tabular}{ll}
\hline \multicolumn{1}{c}{ Themes } & \multicolumn{1}{c}{ Descriptions } \\
\hline $\begin{array}{c}\text { Partnerships Among } \\
\text { Stakeholders }\end{array}$ & $\begin{array}{l}\text { Active partnerships. } \\
\text { EHDI and newborn metabolic screening working closely } \\
\text { together. } \\
\text { Increased collaboration among state agencies. }\end{array}$ \\
Data Systems & $\begin{array}{l}\text { Improved data system; improved tracking and surveillance. } \\
\text { State funding attached to metabolic screening provides some } \\
\text { sunding }\end{array}$ \\
stable funding. \\
Using contract staff; consolidation of staff has improved \\
communication. \\
Datfing \\
Dystem Support \\
state department of education. \\
Leveraging more support from stakeholders and partnerships. \\
Increased support for EHDI at the community level.
\end{tabular}


administrative needs. Some states reported consolidating EHDI program staff to one worksite to improve coordination and communication among staff members.

System support was the final top opportunity, cited by $11 \%$ of respondents. Coordinators reported increased support for the EHDI program through newly established and ongoing collaborative relationships among key stakeholders. Some coordinators reported an increased use of memoranda to define cooperative agreements, operational practices, and the possibility of consolidating services among service providers and state programs.

Other opportunities included public awareness initiatives (9\%), legislation $(8 \%)$, and advisory committee $(3 \%)$. Respondents reported that there are several projects underway to promote EHDI, new legislation initiatives to help with program sustainability, and expansions planned for their advisory committees.

\section{Threats to EHDI Programs}

The most frequently cited threats were funding $(62 \%)$, legislative and regulatory action $(13 \%)$, and staffing $(9 \%)$ (Table 5). Sixty-two percent of the states reported significant challenges to the EHDI program's infrastructure due to inadequate funding and resources. Coordinators reported that state budget cuts and a depressed local economy were the greatest challenge to sustaining the current level of program services. Continued funding shortages have led to program instability, staff shortages, and reduced resources for screening

Table 5. Top three themes for threats

\begin{tabular}{ll}
\hline \multicolumn{1}{c}{ Themes } & \multicolumn{1}{c}{ Descriptions } \\
\hline Funding & Budget cuts. \\
& Reduction in federal funding. \\
& No state funds supporting the program. \\
Legislative \& Regulatory & Current legislation needs to be changed. \\
Action & No audiology reporting or data sharing provisions in \\
& current legislation. \\
& Legislature meets only once every 2 years; takes too long \\
& to pass a bill. \\
Limited staffing. & Limited staffing at the state level with competing \\
priorities. & Staff hiring freeze, resulting in increased work demands \\
for remaining staff.
\end{tabular}


equipment maintenance, travel, and training/staff development. Most states expressed concerns about long-term program viability if continued funding cuts occur at the federal and state levels.

Legislative and regulatory action was cited by $13 \%$ of the state EHDI programs as a potential threat to the program's infrastructure. Coordinators noted the lengthy process to get a bill passed in the state legislature or to modify existing mandates as a significant challenge. Finally, 9\% of coordinators reported staffing as a threat, including limited staffing (especially at the state level), competing priorities, and a staff hiring freeze, resulting in increased work demands for remaining staff.

Four percent of the EHDI coordinators also mentioned the lack or misinformation about EHDI programs. These comments were categorized under the public awareness theme.

\section{TOWS Analysis Matrix}

By applying the TOWS analysis matrix to the themes identified in the SWOT analysis, specific recommendations, or "strategic options," were derived from the internal and external environmental factors identified by the EHDI coordinators. By systematically pairing the four categories of the SWOT analysis, recommendations can be derived to maximize resources and effect positive change. Based on the SWOT themes, 12 individual recommendations emerged to strengthen infrastructure of state EHDI programs (Table 6). To maximize opportunities, building strengths can maximize efficiency and lead to positive change (S-O strategy). For example, by identifying federal and state funding resources, additional staff can be hired to help with identified areas of need within the EHDI program. To overcome weaknesses, opportunities must be pursued (W-O strategy). For example, by utilizing formal agreements and working with collaborative partners, awareness of EHDI program activities and services will increase. Identifying ways to use strengths can reduce vulnerability to threats (S-T strategy). For example, by utilizing the advisory board's connections and support, efforts to strengthen funding sources for specific projects can be explored. Finally, establishing defensive plans will help to prevent weaknesses from making programs and services susceptible to threats (W-T strategy). For example, using the advisory board and relationships with key stakeholders to increase awareness for EHDI activities will limit threats to funding and staff support. From these 12 recommendations, 3 strategies emerged to enhance EHDI program infrastructure. Specifically, states should: 1) use the EHDI advisory committee to establish and expand collaborative agreements with key stakeholders; 2) maximize funding opportunities from local and state resources; and 3) raise public awareness about the purpose and importance of newborn hearing screening, early diagnosis, and early intervention for children with hearing loss. 
Table 6. TOWS analysis matrix for EHDI program infrastructure

\begin{tabular}{|c|c|c|c|}
\hline & \multicolumn{2}{|c|}{ Internal } \\
\hline & & Strengths & Weaknesses \\
\hline \multirow{4}{*}{ 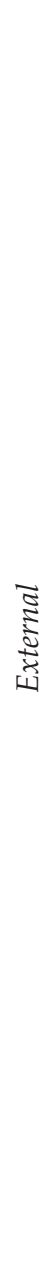 } & \multirow{4}{*}{ 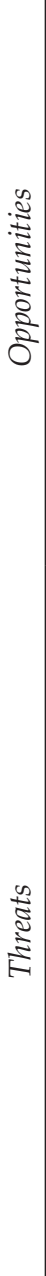 } & S-O Strategies & W-O Strategies \\
\hline & & $\begin{array}{l}\text { 1. Continue to leverage the } \\
\text { advisory committee to build } \\
\text { and strengthen partnerships } \\
\text { and collaborative agreements } \\
\text { among key stakeholders } \\
\text { 2. Increase staff support through } \\
\text { identified federal and state } \\
\text { funding sources. } \\
\text { 3. Strengthen EHDI system } \\
\text { support through formalized } \\
\text { agreements, memoranda of } \\
\text { agreement, and partnerships } \\
\text { with key stakeholders. }\end{array}$ & $\begin{array}{l}\text { 1. Use partnerships among key } \\
\text { stakeholders to lessen the impact } \\
\text { of staff shortages through } \\
\text { consolidation of service delivery. } \\
\text { 2. Use collaboration and formal } \\
\text { agreements with stakeholders to } \\
\text { increase awareness of EHDI } \\
\text { program activities and services. } \\
\text { 3. Leverage partnerships of key } \\
\text { stakeholders to garner support } \\
\text { for improved legislation at the } \\
\text { state level. }\end{array}$ \\
\hline & & S-T Strategies & W-T Strategies \\
\hline & & $\begin{array}{l}\text { 1. Use the advisory committee to } \\
\text { support and organize efforts to } \\
\text { foster improved funding, } \\
\text { especially at the state level. } \\
\text { 2. Through partnerships and } \\
\text { collaborative relationships, } \\
\text { target strategies to recruit and } \\
\text { retain staff. } \\
\text { 3. Use the advisory committee to } \\
\text { organize support to change and } \\
\text { enhance existing state laws and } \\
\text { policies that improve EHDI } \\
\text { service delivery. }\end{array}$ & $\begin{array}{l}\text { 1. Use the advisory committee and } \\
\text { relationships with key stakehold- } \\
\text { ers to increase awareness of EHDI } \\
\text { activities, which limits threats to } \\
\text { funding and staff support. } \\
\text { 2. Identify specific legislative or } \\
\text { policy initiatives that will provide } \\
\text { additional support and reduce } \\
\text { instability. } \\
\text { 3. Determine specific strategies } \\
\text { to increase funding, such as } \\
\text { insurance/Medicaid } \\
\text { reimbursement for some } \\
\text { services, cooperative agreements } \\
\text { with other state agencies, and } \\
\text { changes to state level funding } \\
\text { through the legislative process. }\end{array}$ \\
\hline
\end{tabular}

$\mathrm{S}=$ Strength $\mathrm{W}=$ Weakness $\mathrm{O}=$ Opportunity $\mathrm{T}=$ Threat

\section{Limitations}

Limitations of this study include those inherent in the application of SWOT/TOWS analysis when applied to rapidly changing conditions. SWOT analysis is a subjective tool that explores conditions that are perceived rather 
than objectively measured. In this sense, the SWOT analysis is influenced by the perspectives of those who completed the surveys as well as those who analyzed the responses. Furthermore, SWOT analysis characterizes the situation at a specific point in time. In addition, SWOT analysis, as applied here, resulted in overlapping themes within a category. For example, funding, legislation, and staffing could each be identified as a strength, weakness, opportunity, and threat (see White \& Blaiser, 2011, for additional information regarding the selection of SWOT/TOWS for this study and further comment regarding interpretation and application of findings).

\section{Summary}

Forty-seven EHDI coordinators provided 292 responses about the infrastructure of EHDI programs. Their responses reveal a range of both successes and challenges currently faced by state EHDI programs. While many state coordinators expressed success with obtaining federal and state funding streams, most noted that long term viability of the EHDI program was in jeopardy because of pending or anticipated funding cuts from both state and federal sources. EHDI program advisory committees have been charged with multiple tasks, including program evaluation, relationship building, and increasing public awareness. Many coordinators reported a significant lack of awareness among the general public and key stakeholders, state agency administrators, and legislators. Increasing public awareness - and by extension raising the profile of the state's EHDI program and the positive outcomes associated with early identification - will strengthen the likelihood that additional resources can be obtained.

As state EHDI programs continue to survive despite decreased or restricted funding, more emphasis should be placed on collaborative agreements among key stakeholders, which could maximize opportunities for new funding streams and potentially produce cost savings. The range of services provided by EHDI programs should be publicized - from newborn hearing screening and early diagnosis to the importance of early intervention for children with hearing loss. Lack of awareness of the EHDI program is a continuing threat to its infrastructure; however, by marshalling all available resources, state EHDI coordinators, advisory committees, and partners can garner more attention and increase knowledge about the benefits of early hearing detection and intervention. It is hoped that by raising the profile of the EHDI program among key stakeholders and decision-makers, the necessary resources, funding, and opportunities for success will follow.

\section{Acknowledgement}

The work reported in this article was funded in part by the Maternal and Child Health Bureau under Cooperative Agreement \# U52MC04391 with 
the National Center for Hearing Assessment and Management at Utah State University. The opinions expressed in the article are those of the authors and do not necessarily reflect those of the Bureau.

\section{References}

Allen, K. D., \& Houston, K. T. (2010). Current practices in early intervention: Part and EHDI coordinators' knowledge of communication options for children with hearing loss. Unpublished manuscript, Utah State University. Apuzzo, M. L., \& Yoshinaga-Itano, C. (1995). Early identification of infants with significant hearing loss and the Minnesota Child Development Inventory. Seminars in Hearing, 16, 124-137.

Calderon, R. (2000). Parental involvement in deaf children's education programs as a predictor of child's language, early reading, and socialemotional development. Journal of Deaf Studies and Deaf Education, 5, 140-155.

Centers for Disease Control and Prevention (CDC). (2010a). Annual data: Early hearing detection and intervention (EHDI) program. Retrieved May 24, 2011, from http:/ / www.cdc.gov/ncbddd/hearingloss/ehdi-data.html

Centers for Disease Control and Prevention (CDC). (2010b). Early hearing detection and intervention national goals. Retrieved May 19, 2011, from http:// www.cdc.gov/ncbddd/hearingloss/ehdi-goals.html.

Compton, M. V., Tucker, D. A., \& Flynn, P. F. (2009). Preparation and perception of speech-language pathologists working with children with cochlear implants. Communication Disorders Quarterly, 30(3), 142-154.

Faust, T. E. \& Ditty, K. M. (2010). Financing and sustainability. In L. R. Schmeltz (Ed.), The NCHAM eBook: A resource guide for early hearing detection and intervention (EHDI) (pp. 10-1-10-10). Logan, Utah: National Center for Hearing Assessment and Management. Retrieved May 19, 2011, from www.infant hearing.org/ehdi-ebook/index.html.

Green, D. R., Gaffney, M., Devine, O. \& Grosse, S. D. (2007, March-April). Determining the effect of newborn screening legislation: An analysis of state hearing screening rates. Public Health Reports, 122, 198-205.

Houston, K. T., \& Caraway, T. (2010). For children with hearing loss: The times, they are a-changin. Perspectives on Hearing and Hearing Disorders in Childhood, 20(2), 48-55.

Houston, K. T., \& Perigoe, C. B. (2010). Speech-language pathologists: Vital listening and spoken language professionals. The Volta Review, 110(2), 219-230.

Johnson, H. A. (2004). U.S. deaf education teacher preparation programs: A look at the present and a vision for the future. American Annals of the Deaf, 149(2), 75-91.

Joint Committee on Infant Hearing (JCIH). (2007). Year 2007 position statement: Principles and guidelines for early hearing detection and intervention programs. Pediatrics, 120(4), 898-921. 
Leigh, G., Schmulian-Taljaard, D., \& Poulakis, Z. (2010). Newborn hearing screening. In C. Driscoll \& B. McPherson, (Eds.), Newborn screening systems: The complete narrative (p. 95-115). San Diego, CA: Plural Publishing.

Lenihan, S. (2010). Trends and challenges in teacher preparation in deaf education. The Volta Review, 110(2), 117-128.

Luckhurst, J. A. (2008). Professional preparedness for provision of auditory oral programs to children with hearing loss: Results of a survey. Perspectives on Aural Rehabilitation and Its Instrumentation, 15, 2-18.

Mayne, A. M., Yoshinaga-Itano, C., \& Sedey, A. L. (1998). Receptive vocabulary development of infants and toddlers who are deaf or hard of hearing. The Volta Review, 100(5), 29-52.

McCollister, F. P. (2010). Bringing it all together. In L. R. Schmeltz (Ed.), The NCHAM eBook: A resource guide for early hearing detection and intervention (EHDI), (pp. 12-1-12-12). Logan, Utah: National Center for Hearing Assessment and Management. Retrieved April 27, 2011, from www.infan thearing.org/ehdi-ebook/index.html.

Moeller, M. P. (2000). Early intervention and language development who are deaf and hard of hearing. Pediatrics, 106(3), E43.

Moeller, M. P., White, K. R., \& Shisler, L. (2006). Primary care physicians' knowledge, attitudes, and practices related to newborn hearing screening. Pediatrics, 118(4), 1357-1370.

National Center for Hearing Assessment and Management (NCHAM). (2004). 2004 state EHDI survey. Retrieved May 19, 2011, from http://www.infant hearing.org/survey/2004statesurvey/index.html.

National Center for Hearing Assessment and Management (NCHAM). (2010). State EHDI/UNHS mandates: Summary table. Retrieved May 17, 2011, from http://www.infanthearing.org/legislative/summary/index.html.

Nemes, J. (2006). Success of infant screening creates urgent need for better follow-up. The Hearing Journal, 59(4), 21-28.

Pipp-Siegel, S., Sedey, A. L., VanLeeuwen, A. M., \& Yoshinaga-Itano, C. (2003). Mastery motivation and expressive language in young children with hearing loss. Journal of Deaf Studies and Deaf Education, 8, 133-145.

Robbins, A. M. \& Caraway, T. (2010). Missing the mark in early intervention for babies who are hard of hearing or deaf learning spoken language. Perspectives on Hearing and Hearing Disorders in Childhood, 20(2), 41-47.

Sieminski, L. R. (2010). The audiologist's role in early intervention. The Hearing Journal, 63(1), p. 35.

Shulman, S., Besculides, M., Saltzman, A., Ireys, H., \& White, K. R. (2010). Evaluation of the universal newborn hearing screening and intervention program. Pediatrics, 126, S19-S27.

Vernon, J. (2007). Preparing professionals for a changing landscape. Volta Voices, 14(6), 18-21.

White, K. (2003). The current status of EHDI programs in the United States. Mental Retardation and Developmental Disabilities Research Reviews, 9, 79-88. 
White, K. (2007). Early intervention for children with permanent hearing loss: Finishing the EHDI revolution. The Volta Review, 106(3), 237-258.

White, K. (2008). Newborn hearing screening. In J. R. Madell and C. Flexer (Eds.), Pediatric audiology: Diagnosis, technology, and management (pp. 31-41). New York: Thieme.

White, K., \& Blaiser, K. (2011). Strategic planning to improve early hearing detection and intervention programs. The Volta Review, 111(2), 83-108.

White, K. R., Forsman, I., Eichwald, J. \& Muñoz, K. (2010a). The foundations and evolution of EHDI. In L. R. Schmeltz (Ed.), The NCHAM eBook: A resource guide for early hearing detection and intervention (EHDI) (pp. 1-1-1-18). Logan, Utah: National Center for Hearing Assessment and Management. Retrieved from http:/ /www.infanthearing.org/ehdi-ebook/index.html.

White, K. R., Forsman, I., Eichwald, J., \& Muñoz, K. (2010b). The evolution of early hearing detection and intervention programs in the United States. Seminars in Perinatology, 31(2), 170-189.

Winston, R. (2008). Introducing the newborn hearing screening training curriculum. Perspectives on Hearing and Hearing Disorders in Childhood, 18, 39-43.

Yoshinaga-Itano, C. (2001). The social-emotional ramifications of universal newborn hearing screening: Early identification and intervention of children who are deaf or hard of hearing. In Proceedings of the Second International Pediatric Conference: A Sound Foundation Through Early Amplification. Stafa, Switzerland: Phonak.

Yoshinaga-Itano, C., Sedey, A. L., Coulter, D. K., \& Mehl, A. L. (1998). Language of early- and later-identified children with hearing loss. Pediatrics, 102, 1161-1171. 


\title{
Data Management in the EHDI System
}

\author{
Tamala S. Bradham, Ph.D., CCC-A; Jeff Hoffman, M.S., CCC-A; and \\ K. Todd Houston, Ph.D., CCC-SLP, LSLS Cert. AVT
}

State coordinators of early hearing detection and intervention (EHDI) programs completed a strengths, weaknesses, opportunities, and threats, or SWOT, analysis that examined 12 areas within EHDI programs. Forty-seven coordinators listed 242 items in the area of data management, and themes were identified in each category. A threats, opportunities, weaknesses, and strengths, or TOWS, analysis yielded 12 recommendations that focused on similar themes. Out of these 12 recommendations, 3 strategies emerged: 1) address problems related to sharing data and/or link data systems; 2) maintain and/or upgrade existing systems that are flexible, web-based, streamline data entry, and accessible by those who need it; and 3) implement a quality monitoring program from the data collected to improve systems. Addressed appropriately, these recommendations can strengthen data management in the EHDI system.

\section{Introduction}

Newborn hearing screening prior to hospital discharge in now a standard of care in the United States (Maternal and Child Health Bureau [MCHB], 2010). According to White, Forsman, Eichwald, and Muñoz (2010), 43 states plus the District of Columbia have enacted universal newborn hearing screening (UNHS) legislation, and states with legislation are more likely to screen $95 \%$ or more infants for hearing loss than those without UNHS. Although some might argue that this level of participation is remarkable, it is surprising that after nearly four decades of debating the value of early identification of hearing loss

Tamala S. Bradham, Ph.D., CCC-A, is an Assistant Professor in the School of Medicine, Department of Hearing and Speech Sciences, at Vanderbilt University. Jeff Hoffman, M.S., CCC-A, is a Senior Research Scientist - Audiologist in the National Center for Hearing Assessment and Management at Utah State University. K. Todd Houston, Ph.D., CCC-SLP, LSLS Cert. AVT, was an Assistant Professor in the Department of Communicative Disorders and Deaf Education at Utah State University at the time of this study and is currently at The University of Akron. Correspondence concerning this manuscript should be addressed to Dr. Bradham at tamala.bradham@vanderbilt.edu. 
not all states have enacted legislation mandating early hearing detection and intervention (EHDI) programs.

Universal screening, diagnosis, and intervention programs are under continued scrutiny from the medical profession, legislators, health reimbursement industry, and consumers. In 1994, Bess and Paradise (1994) questioned the National Institutes of Health (NIH) 1993 consensus statement primarily on the grounds that there was insufficient evidence to support newborn hearing screening. Specifically, they identified the need for more research in the following areas: 1) qualifications of the personnel who administer the screening, 2) effectiveness of screening procedures, 3) the validity and reliability of the screening equipment, 4) cost-effectiveness, 5) effectiveness of treatment dependent on the age of identification, and 6) compliance to follow-through. In 2001, the U.S. Preventive Services Task Force (USPSTF) released a thorough review of UNHS and concluded the evidence was "insufficient to recommend for or against routine screening of newborns for hearing loss during the postpartum hospitalization" (USPSTF, 2001). Furthermore, this report did not find any evidence to determine whether earlier treatment resulted in improvements in speech and language skills. The review did, however, report that newborn hearing screening led to earlier identification and treatment of infants with hearing loss. In a later review in 2008, the USPSTF upgraded UNHS to a Grade B and advised the following (USPSTF, 2008):

- All infants should be screened by 1 month.

- Infants who don't pass should undergo audiological evaluations for confirmation of hearing loss by 6 months of age.

- Early intervention services should be established for the child that included sensory devices, medical evaluations, and communication assessment and therapy.

Listing UNHS and early intervention as national priorities (Centers for Disease Control and Prevention [CDC], 2010a; U.S. Department of Health and Human Services [DHHS], 2010) has had an impact on the ability to collect essential evidence to support EHDI programs. Clearly defining benchmarks, goals, and objectives helps states report a consistent message about the effectiveness of their EHDI programs. Healthy People 2020 (DHHS, 2010) clearly defined eight goals under their hearing section, with the first goal being:

"Increase the proportion of newborns who are screened for hearing loss by no later than age 1 month, have audiological evaluation by age 3 months, and are enrolled in appropriate intervention services no later than age 6 months." 
The CDC (2010b) also monitors the effectiveness of EHDI and reports the following goals:

- Goal 1. All newborns will be screened for hearing loss before 1 month of age, preferably before hospital discharge.

- Goal 2. All infants who screen positive will have a diagnostic audiological evaluation before 3 months of age.

- Goal 3. All infants identified with hearing loss will receive appropriate early intervention services before 6 months of age (medical, audiological, and early intervention).

- Goal 4. All infants and children with late onset, progressive, or acquired hearing loss will be identified at the earliest possible time.

- Goal 5. All infants with hearing loss will have a medical home as defined by the American Academy of Pediatrics.

- Goal 6. Every state will have a complete EHDI Tracking and Surveillance System that will minimize loss to follow-up.

- Goal 7. Every state will have a comprehensive system that monitors and evaluates the progress towards the EHDI Goals and Objectives.

Goal 6 specifically targets the need for a quality data management system. Objectives include a comprehensive data system, explicit policies and procedures, privacy and confidentiality procedures, a goal to enter all live births in system, explain risk factors for hearing loss, provide newborn hearing screening results, and include a reporting mechanism for health providers (CDC, 2010b).

To further stress the importance of data and the impact it has on policies, insurance reimbursement, and access to care, the Joint Committee on Infant Hearing $(\mathrm{JCIH})$ also supports the necessity of having an infrastructure in place for collecting and monitoring quality data (JCIH, 2007). JCIH recommends several initiatives that involve data management, specifically:

- A limited national database.

- Demographics of neonatal hearing loss, including prevalence and etiology.

- Assist both primary health care providers and state health agency in measuring quality indicators.

- Provide tools for programmatic evaluation of benchmarks.

- Must be stable, sustainable, timely, secure, and accurate.

- Monitoring of data management system must be ongoing.

Furthermore, federal funding decisions are linked with overall performance data (Government Performance and Results Act, 1993), thus access to federal funding requires a mechanism for data collection, analysis, and distribution of the results. 
To minimize scrutiny when comparing state outcomes to national benchmarks and goals, it is essential for the survival of EHDI to have clean, accessible, and quality data. National goals and benchmarks provide a framework to states on how to monitor the effectiveness of their EHDI programs. It is essential, however, that EHDI programs monitor how they are doing and continue to plan ahead. Each state has an EHDI coordinator whose responsibilities include the development of statewide systems that meet these national priorities. As part of their role, EHDI coordinators are charged with establishing partnerships with hospitals, audiologists, physicians, and early intervention systems; implementing protocols for effective management of early identification and intervention of infants and toddlers with hearing loss; creating family support systems that are culturally and linguistically appropriate; and sustaining a data management system for tracking the effectiveness of the program.

The purpose of this study was to provide a systematic review of data management systems using a strengths, weaknesses, opportunities, and threats, or SWOT, and a threats, opportunities, weaknesses, and strengths, or TOWS, analysis to determine the current EHDI practices in data management, and to identify factors to improve program effectiveness that lead to better outcomes for infants with hearing loss.

\section{Methods}

Fifty-one EHDI coordinators were asked to complete an online survey. Related to data management services, the survey asked for the EHDI coordinator to report at least 1 item in the following four areas to assist with strategic planning: strength, weakness, opportunity, and threat. The responses obtained were reviewed by a panel of experts in the field and categorized into common themes in each of these four strategic planning areas. To generate recommendations from the SWOT analysis, a TOWS matrix was used to match identified strengths with opportunities (S-O strategy), strengths with threats (S-T strategy), weaknesses with opportunities (W-O strategy), and weaknesses with threats (W-T strategy). For an in-depth review of the methodology, the reader is referred to White and Blaiser, 2011.

\section{Results}

Forty-seven EHDI coordinators (92\%) completed the data management area of the SWOT questionnaire. The respondents generated 69 items in the strength category, 70 items in the weaknesses category, 49 items in the opportunities category, and 54 items in the threats category for a total of 242 responses. Based on the responses, several specific themes were generated for each category: 8 for strengths, 9 for weaknesses, 5 for opportunities, and 6 for threats (Table 1). 
Table I. Frequency of themes within each SWOT category $(n=242)$

\begin{tabular}{|c|c|c|}
\hline SWOT category & Themes & Frequency n $(\%)$ \\
\hline Strengths $(\mathrm{n}=69)$ & $\begin{array}{l}\text { Data Collection Practices/Development } \\
\text { Technology/Web-Based Connections } \\
\text { Staffing/Resources/Training } \\
\text { Experience with Data Systems } \\
\text { Legislation/Policy } \\
\text { Miscellaneous/No Comments } \\
\text { Communication/Collaboration } \\
\text { Timeliness of Reporting Data }\end{array}$ & $\begin{array}{l}24(35 \%) \\
19(28 \%) \\
13(19 \%) \\
3(4 \%) \\
3(4) \% \\
3(4 \%) \\
3(4 \%) \\
1(1 \%)\end{array}$ \\
\hline Weaknesses $(\mathrm{n}=70)$ & $\begin{array}{l}\text { Data Collection Practices/Development } \\
\text { Staffing/Resources/Training } \\
\text { Barriers to Using Database for System Change } \\
\text { Multiple Database Systems } \\
\text { Miscellaneous/No Comments } \\
\text { Lack of Access to Existing Database } \\
\text { Lack of Database } \\
\text { Timeliness } \\
\text { Legislation/Policy }\end{array}$ & $\begin{array}{l}17(24 \%) \\
9(13 \%) \\
9(13 \%) \\
8(11 \%) \\
8(11 \%) \\
6(9 \%) \\
5(7 \%) \\
5(7 \%) \\
3(4 \%)\end{array}$ \\
\hline Opportunities $(n=49)$ & $\begin{array}{l}\text { Making Improvements to Existing Database } \\
\text { Data Collection Practices } \\
\text { Technology/Web-Based Connections } \\
\text { Staffing/Resources/Training } \\
\text { Miscellaneous/No Comments }\end{array}$ & $\begin{array}{l}17(35 \%) \\
10(20 \%) \\
10(20 \%) \\
10(20 \%) \\
2(4 \%)\end{array}$ \\
\hline Threats $(\mathrm{n}=54)$ & $\begin{array}{l}\text { Funding } \\
\text { Staffing/Resources/Training } \\
\text { Data Collection Practices/Development } \\
\text { Miscellaneous/No Comment } \\
\text { Communication/Collaboration } \\
\text { Legislation/Policy }\end{array}$ & $\begin{aligned} & 22(41 \%) \\
& 10(19 \%) \\
& 9(17 \%) \\
& 7(13 \%) \\
& 3(6 \%) \\
& 3(6 \%)\end{aligned}$ \\
\hline
\end{tabular}

Each category also had a miscellaneous section that included no comment, $\mathrm{N} / \mathrm{A}$, or items that did not fit within the themes identified.

\section{Strength Characteristics}

The most frequently cited strengths were data collection practices (35\%), technology and web-based systems (28\%), and staffing/resources/training $(19 \%)$ (Table 2). Thirty-five percent of respondents noted that linking data systems across multiple agencies (e.g., vital records, birth certificates, early intervention) and providers was a strength. Using commercially available systems that were flexible and allowed for reporting as well as web-based data management systems were also considered a strength by $28 \%$ of respondents. Nineteen percent of EHDI coordinators reported having appropriate 
Table 2. Top three themes for strengths

\begin{tabular}{cl}
\hline \multicolumn{1}{c}{ Themes } & \multicolumn{1}{c}{ Description } \\
\hline Data Collection Practices & Linkage of data system to allow for data. \\
& Tracking the "right" data for reporting and monitoring. \\
& Compliance in reporting data matching. \\
Technology and Web-Based & Use of commercially available web-based systems. \\
Systems & Use of state systems that link data sets. \\
& Can be used by all stakeholders (hospitals, audiologists, \\
& medical homes, early intervention, etc.). \\
Resources & Employ full-time epidemiologists (not a shared \\
& position). \\
& 24-hour IT support and vendor support. \\
& Knowledgeable data entry personnel. \\
\hline
\end{tabular}

resources including, but not limited to, 24-hour information technology (IT) and vendor support, appropriate staffing for data entry and analysis, and funding for updating the data management system as strengths.

Other strengths included staff experience with data systems (4\%), legislative and policy support (4\%), communication and collaboration with hospitals, audiologists, and health departments $(4 \%)$, and timeliness of reporting data $(1 \%)$.

\section{Weakness Characteristics}

The most frequently cited weaknesses were data collection practices $(24 \%)$, barriers with data management systems (13\%), and staffing/resources/training issues (13\%) (Table 3). Twenty-four percent of EHDI coordinators noted

Table 3. Top three themes for weaknesses

\begin{tabular}{cl}
\hline \multicolumn{1}{c}{ Themes } & \multicolumn{1}{c}{ Description } \\
\hline Data Collection Practices & Limited flexibility of data systems. \\
& Not web-based. \\
& Receiving data in a timely manner. \\
Barriers with Data & Lack real-time data entry. \\
Management Systems & Lack of linkage between data systems. \\
& Difficult to navigate, query data, and run reports. \\
Staffing Issues & Turnover and lack of knowledgeable data entry staff. \\
& Limited staff have access to data system for reports. \\
& No one to analyze data. \\
\hline
\end{tabular}


that the areas of weakness for data collection included limited flexibility of the data systems, not having a web-based system, and the lack of real-time data entry. Thirteen percent of respondents reported they did not have a statewide database, or that their system only reported aggregated data and did not allow for tracking individual infants and toddlers. Having access to the data management system was also another reported issue by $13 \%$ of respondents. Specifically, not all providers have access to the state data management system, limited staff members who could run reports, and a lack of consistent data entry was cited.

Of interest, many state coordinators (11\%) reported having multiple data management systems to track variables not captured in their primary system (e.g., spreadsheets, word documents, or other self-made databases). Since reporting of data is not mandated in many states, EHDI coordinators reported difficulties collecting data $(7 \%)$. Other reported weaknesses included lack of access to existing database ( $9 \%)$, timeliness of data entry $(7 \%)$, and lack of legislative or policy support (4\%).

\section{Opportunities for EHDI Programs}

The most frequently cited opportunities were improving current data system $(35 \%)$, data collection practices $(20 \%)$, web-based systems $(20 \%)$, and staffing/resources/training (20\%) (Table 4). Thirty-five percent of respondents received funding to update, expand, and link their EHDI data management system to other state systems. Twenty percent of respondents also noted that addressing the Family Educational Rights and Privacy Act (FERPA) and Health Insurance Portability and Accountability Act (HIPPA) laws to allow for data sharing and/or linkage of systems improved data management. Having knowledgeable, available key staff to help with IT, data entry, data analysis,

Table 4. Top three themes for opportunities

\begin{tabular}{|c|c|}
\hline Themes & Description \\
\hline Improving Current Data Systems & $\begin{array}{l}\text { Linkage of data system to allow for data matching. } \\
\text { Expanding data fields to allow for better tracking, } \\
\text { reporting, and monitoring. } \\
\text { Web-based system (no longer paper-based). }\end{array}$ \\
\hline Data Collection Practices & $\begin{array}{l}\text { Audiologists involved in data collection. } \\
\text { Flexibility of data systems. } \\
\text { Resolving issues about FERPA and HIPPA to allow } \\
\text { for linkage of data systems. }\end{array}$ \\
\hline Web-Based Systems & $\begin{array}{l}\text { Linking state and vendor data systems. } \\
\text { Consistent IT support. } \\
\text { Having a web-based system. }\end{array}$ \\
\hline
\end{tabular}


and reporting were also noted as essential for a data management program by $20 \%$ of EHDI coordinators.

\section{Threats to EHDI Programs}

The most frequently cited threats were funding (41\%), staffing/resources/ trainings (19\%), and data collection practices (17\%) (Table 5). Overwhelmingly, $41 \%$ of respondents reported that funding, budget cuts, and a pending fiscal crisis were all threats to their program. Several EHDI coordinators questioned the future existence of EHDI programs due to a fiscal crisis faced by the U.S. government as well as their own state government. Nineteen percent of respondents indicated that a lack of appropriate resources, such as not having enough staff and IT support to maintain the data systems and to keep up with software upgrades and future data linkage projects. Due to FERPA and HIPPA laws, $17 \%$ of EHDI coordinators cited difficulties obtaining data from other state run programs. Without having access to this data, EHDI coordinators are challenged to appropriately manage their programs. Other threats included poor communication/collaboration (6\%) and limited legislative and policy support (3\%).

Table 5. Top three themes for threats

\begin{tabular}{|c|c|}
\hline Themes & Description \\
\hline Funding & $\begin{array}{l}\text { Fiscal crisis. } \\
\text { EHDI program would end if Federal funding stopped. } \\
\text { Budget cuts. }\end{array}$ \\
\hline Resources & $\begin{array}{l}\text { Lack of IT support. } \\
\text { Staff time for data entry and monitoring of data. } \\
\text { Keep up-to-date with technology that changes so quickly. }\end{array}$ \\
\hline Data Collection Practices & $\begin{array}{l}\text { Privacy issues that don't allow for linkage or data sharing. } \\
\text { Current data systems do not provide the information } \\
\text { needed for annual reports to CDC, state, and/or } \\
\text { stakeholders/providers. } \\
\text { Holding staff/providers accountable for accurate, } \\
\text { real-time data entry. }\end{array}$ \\
\hline
\end{tabular}

\section{TOWS Analysis Matrix}

By applying the TOWS analysis matrix to the themes identified in the SWOT analysis, specific recommendations, or "strategic options," were derived from the internal and external environmental factors identified by the EHDI coordinators. By systematically pairing the four categories of the SWOT analysis, recommendations can be derived to maximize resources and effect positive change. Based on the SWOT themes, 12 individual recommendations 
emerged (Table 6). To maximize opportunities, turning to strengths can maximize efficiency and lead to positive change (S-O strategy). For example, creating or modifying a data management system that is web-based, links to other systems, and creates automatic reports can reduce staff time needed to manage the system. To overcome weaknesses, pursue opportunities (W-O strategy). For example, by resolving issues around data sharing and confidentiality (e.g., address HIPPA and FERPA laws), EHDI coordinators will be able to better track and follow-up with the children they serve. Identifying ways to use strengths will ultimately reduce vulnerability to threats (S-T strategy). For example, allowing community providers access to the data management system in which to enter their data will save needed resources in the EHDI program (e.g., fax paper and manual data entry). Finally, establishing defensive plans will help prevent weaknesses from making policies and services susceptible to

Table 6. TOWS analysis matrix for data management

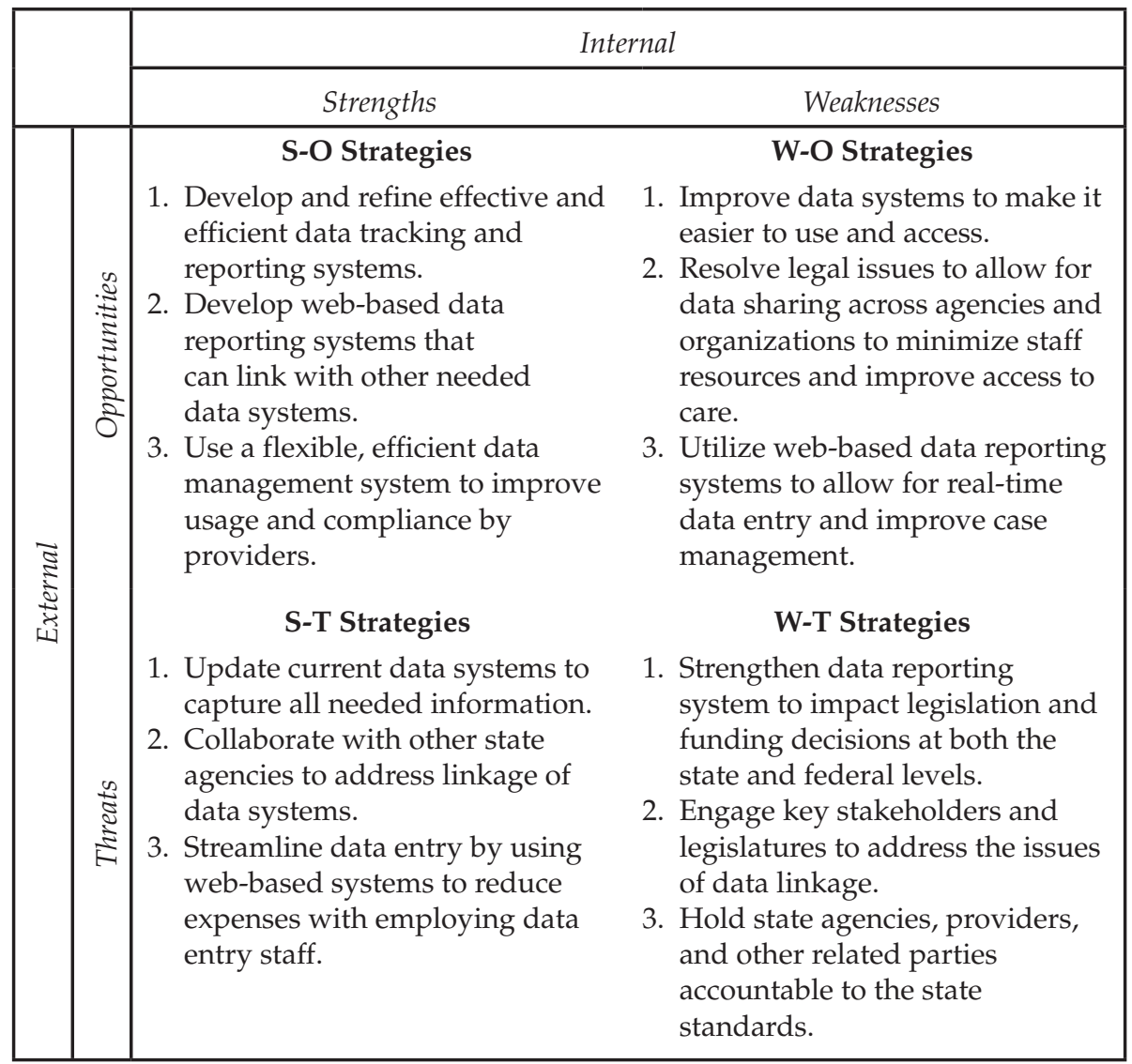

$\mathrm{S}=$ Strength $\mathrm{W}=$ Weakness $\mathrm{O}=$ Opportunity $\mathrm{T}=$ Threat 
threats (W-T strategy). For example, strengthening the data management system with quality data will help EHDI programs tell their stories on how effective they are in meeting the needs of children with hearing loss and their families. This may impact legislative efforts, funding, and state and national priorities. Out of these 12 recommendations, 3 strategies emerged that would help state EHDI programs effectively manage their data management systems. Specifically, programs should: 1) address how they will share data and/or link data systems; 2) maintain and/or upgrade existing systems that are flexible, web-based, streamlined data entry, and accessible by those who need it; and 3) implement a quality monitoring program from the data collected to improve systems.

\section{Limitations}

Limitations of this study include those inherent in the application of SWOT / TOWS analysis when applied to rapidly changing conditions. SWOT analysis is a subjective tool that explores conditions that are perceived rather than objectively measured. In this sense, the SWOT analysis is influenced by the perspectives of those who completed the surveys as well as those who analyzed the responses. Furthermore, SWOT analysis characterizes the situation at a specific point in time. In addition, SWOT analysis, as applied here, resulted in overlapping themes within a category. For example, data collection, staffing/ resources/trainings, data practices, and development could each be identified as a strength, weakness, opportunity, and threat (see White \& Blaiser, 2011, for additional information regarding the selection of SWOT/TOWS for this study and further comment regarding interpretation and application of findings).

\section{Summary}

While many states have data management systems in place, there are still areas that need improvement. Integrating data management systems to allow key stakeholders access to the system to report their data (e.g., hospitals, audiologists, medical homes, early interventionists, speech-language pathologist) while being in compliance with privacy laws is a significant challenge. Currently, no state has reported a successful resolution to this challenge. Furthermore, due to time constraints and funding issues, state EHDI coordinators report having limited resources to ensure the quality of the data entry and/or reporting. By focusing on these items identified by the TOWS analysis, states will be able to develop action plans to address these needs.

Data management systems are essential for the survival of EHDI programs. Without adequate data, accountability and liability issues will exist. States must report their data to receive ongoing funding to support their EHDI programs. With quality data reporting, states can identify ways to improve their programs and make positive changes that ultimately affect the outcomes of the child and his/her family - who, ultimately, are the beneficiaries of this system. 


\section{Acknowledgement}

The work reported in this article was funded in part by the Maternal and Child Health Bureau under Cooperative Agreement \# U52MC04391 with the National Center for Hearing Assessment and Management at Utah State University. The opinions expressed in the article are those of the authors and do not necessarily reflect those of the Bureau.

\section{References}

Bess, F., \& Paradise, J. L. (1994). Universal screening for infant hearing impairment: Not simple, not risk-free, not necessarily beneficial, and not presently justified. Pediatrics, 93(2), 330-334.

Centers for Disease Control and Prevention (CDC). (2010a). EHDI hearing screening and follow-up survey. Retrieved May 19, 2011, from http://www. cdc.gov/ncbddd/EHDI/data.htm.

Centers for Disease Control and Prevention (CDC). (2010b). Early hearing detection and intervention (EHDI) national goals. Retrieved May 19, 2011, from http://www.cdc.gov/ncbddd/hearingloss/ehdi-goals.html.

Government Performance and Results Act (GPRA) of 1993, Pub L No. 103-62. Joint Committee on Infant Hearing (JCIH). (2007). Year 2007 position statement: Principles and guidelines for early hearing detection and intervention programs. Pediatrics, 120(4), 898-921.

Maternal and Child Health Bureau (MCHB). (2010). Universal newborn hearing screening. Retrieved May 19, 2011, from http://mchb.hrsa.gov/pro grams/specialneeds/unhs.htm.

U.S. Department of Health and Human Services (DHHS). (2010). Healthy people 2020: Hearing and other sensory or communication disorders. Retrieved May 19, 2011, from http:/ / www.healthypeople.gov/2020/topicsobjectives2020/ overview.aspx? topicid $=20$.

U.S. Preventive Services Task Force (USPSTF). (2001). Newborn hearing screening: Recommendations and rationale. Retrieved May 19, 2011, from http://www.ahrq.gov/clinic/3rduspstf/newbornscreen/newhearrr.htm.

U.S. Preventive Services Task Force (USPSTF). (2008). Universal screening for hearing loss in newborns: U.S. Preventive Services Task Force recommendation statement. Pediatric, 122(1), 143-148.

White, K., \& Blaiser, K. (2011). Strategic planning to improve early hearing detection and intervention programs. The Volta Review, 111(2), 83-108.

White, K., Forsman, I., Eichwald, J., \& Muñoz, K. (2010). The evolution of early hearing detection and intervention programs in the United States. Seminars in Perinatology, 31(2), 170-189. 



\title{
Fostering Quality Improvement in EHDI Programs
}

\author{
Tamala S. Bradham, Ph.D., CCC-A; Jeff Hoffman, M.S., CCC-A; \\ K. Todd Houston, Ph.D., CCC-SLP, LSLS Cert. AVT; and \\ Gayla Hutsell Guignard, M.A., CCC-A/SLP, LSLS Cert. AVT
}

State coordinators of early hearing detection and intervention (EHDI) programs completed a strengths, weaknesses, opportunities, and threats, or SWOT, analysis that consisted of 12 evaluative areas of EHDI programs. For the quality improvement area, a total of 218 items were listed by 47 EHDI coordinators, and themes were identified in each category. A threats, opportunities, weaknesses, and strengths, or TOWS, analysis matrix yielded 12 recommendations that focused on similar themes. Out of these 12 recommendations, 3 strategies emerged: 1) upgrade data management systems that will generate automated reports based on the state benchmarks coordinators wish to measure; 2) quality monitoring should be integrated into the state system at all levels and not as a separate job; and 3) all quality measures should be publically posted to hold the EHDI system accountable. Addressed appropriately, these recommendations can strengthen quality improvement in state EHDI programs.

\section{Introduction}

Early hearing detection and intervention (EHDI) programs are a vital publicprivate partnership in screening, identifying, and providing appropriate early intervention services to infants and toddlers with permanent hearing loss.

Tamala S. Bradham, Ph.D., CCC-A, is an Assistant Professor in the School of Medicine, Department of Hearing and Speech Sciences, at Vanderbilt University. Jeff Hoffman, M.S., CCC-A, is a Senior Research Scientist - Audiologist in the National Center for Hearing Assessment and Management at Utah State University. K. Todd Houston, Ph.D., CCC-SLP, LSLS Cert. $A V T$, was an Assistant Professor in the Department of Communicative Disorders and Deaf Education at Utah State University at the time of this study and is currently at The University of Akron. Gayla Hutsell Guignard, M.A., CCC-A/SLP, LSLS Cert. AVT, is the Program Director of Indiana's Early Hearing Detection \& Intervention (EHDI) program in the Department of Genomics \& Newborn Screening at the Indiana State Department of Health. Correspondence concerning this manuscript should be addressed to Dr. Bradham at tamala.bradham@vanderbilt.edu. 
Healthy People 2020 (U.S. Department of Health and Human Services [DHHS], 2010) and the Centers for Disease Control and Prevention (CDC, 2010a) have both stated that newborn hearing screening, identification, and early intervention are national priorities. Today, there are 43 states and U.S. territories that mandate newborn hearing screening (White, Foresman, Eichwald, \& Muñoz, 2010 ), resulting in $97 \%$ of newborns being screened for hearing loss in their local hospital or birthing center (CDC, 2010b).

For the best outcomes, each step within the EHDI system must be able to provide services responsibly and in a timely manner, document all aspects of care, and provide ongoing communication to all appropriate stakeholders. The effectiveness of the system depends on the quality of each step (Finitzo \& Grosse, 2003). To develop and maintain quality programs, each step must be assessed on an ongoing basis and the results of those assessments reported to collaborative partners within the EHDI system. Based on these results, the stakeholders can then implement actionable items for improvement.

Recognizing the importance of quality enhancement within EHDI, the Joint Committee of Infant Hearing (JCIH) 2007 Position Statement recommended that "states should implement data-management and tracking systems as part of an integrated child health information system to monitor the quality of EHDI services and provide recommendations for improving systems of care" (p. 899). The CDC (2010b) also incorporated two quality enhancement goals out of their seven goals for EHDI. Specifically, goal number six states that "every state will have a complete EHDI Tracking and Surveillance System that will minimize loss to follow-up"(p. 9-10) and goal seven states that "Each state EHDI program will develop a program evaluation plan in collaboration with the program Advisory Committee to ensure progress towards national and state program goals and objectives" (p. 10).

\section{Quality Enhancement Programs}

A cursory review of the term "quality" leads to a range of subjective definitions and applications. For example, in quality enhancement programs, a variety of terms are used interchangeably, such as quality management, quality control, quality assurance, quality improvement, continuous quality improvement, quality assessment, and quality monitoring.

\section{Quality Management}

With a quality management program, the systems put into place permeate the entire organization as they are being implemented. The focus is on improving the quality of services provided to satisfy the end user. For EHDI, the quality management program would ultimately affect the child, the end user of the system. That is, was the child's hearing loss identified in a timely manner? Or did the early intervention program produce the desired outcome(s)? Quality 
management often includes three main components: quality control, quality assurance, and quality improvement.

Quality Control. Quality control emphasizes the testing and treatment to uncover problems and has appropriate reporting mechanisms in place (Finitzo \& Grosse, 2003). For example, does the hospital and/or audiologist have a process of checking testing equipment prior to test administration to make sure the equipment is functional and will yield appropriate test results? If a child is not making appropriate progress in meeting his/her early intervention goals, is there a system in place to review essential components known to contribute to overall success (i.e., hearing aids appropriately set, family regularly attending treatment sessions, is the professional who is providing the service qualified to do so, etc.). Quality control helps to address problems as they occur to minimize unsatisfactory results later on in the system.

Quality Assurance. Quality assurance is a systematic process of monitoring and evaluating all aspects of the program to ensure certain standards are being met and identifies problems that can be resolved in a timely manner (Finitzo \& Grosse, 2003). For example, JCIH (2007) has recommended a 1-3-6 model where the child has his/her hearing screened by 1 month of age, audiological diagnostic testing for confirmation of hearing status by 3 months of age, and early intervention in place by 6 months of age (for those who are diagnosed with hearing loss). Within a quality assurance program, the 1-3-6 model would serve as the benchmark for evaluating the program to ensure standards are being met. The quality assurance project may focus on addressing loss to follow-up if the state program is not meeting the 1-3-6 model.

Quality Improvement. Quality improvement evaluates the system both retrospectively and prospectively to ensure standards are being met. By looking at where a system is currently, procedures can be put into place to improve the system. Quality improvement programs help to prevent errors from occurring. For example, when an infant does not pass a newborn hearing screening, the manner by which the information is delivered to the family will impact their decision to return for follow-up testing. If the person who delivers the news minimizes the results, there is a greater likelihood that the family will not return for additional testing. Some, however, will argue that if you tell the family of the results, you may cause psychological and bonding issues between the family and the baby. In essence, how the information is conveyed has an impact on the outcome. A quality improvement project would serve to standardize information delivery of the screening results to the family to ensure appropriate follow-up in a timely manner without any potential harmful psychological effects to the family or the baby.

\section{Continuous Quality Improvement}

Many hospitals, medical practices, and audiological practices have ongoing continuous quality improvement (CQI) programs. Accrediting agencies, 
like the Joint Commission on the Accreditation of Healthcare Organizations (JCAHO), require CQI programs. CQI programs focus on the "process" rather than the individual, and promote the change or improvement of most systems. By building on traditional quality management programs, this model emphasizes the organization and the systems rather than the individual or finding fault. Continuously monitoring and evaluating EHDI programs ensures that quality is a part of the process throughout the program and will ultimately help the programs obtain desired outcomes.

\section{Quality Assessment and Quality Monitoring}

Other terms used in quality enhancement programs include quality assessment and quality monitoring. Quality assessment is a specific measurement of quality in which a comparison is made to an established criteria or standard of care. Quality monitoring is tracking your measurements of quality over time.

For EHDI, quality enhancement programs are areas that deal with how states use data, staff, and other resources for quality improvement, program evaluations, and projects. Within each state EHDI program, "an ongoing evaluation and monitoring process should focus on clearly defined objectives, measureable performance indicators, data collection that is not burdensome, rapid analysis and reporting of results, and use of results by decision makers to fine time procedures" (Finitzo \& Grosse, 2003). JCIH (2007) provides clearly defined quality indicators for each state to use for their quality enhancement program:

- Screening:

- 95\% of all newborn infants (age adjusted for preterm infants) who complete screening by 1 month of age.

- Less than $4 \%$ of all newborn infants who fail initial screening and subsequent rescreening before comprehensive audiological evaluation.

- Confirmation of hearing loss:

- $90 \%$ of all infants who fail the initial screening and any subsequent screenings should complete a comprehensive audiological evaluation by 3 months of age.

- 95\% of infants who families elect amplification should receive them within 1 month of confirmation of hearing loss.

- Early intervention:

- 90\% of infants with confirmed hearing loss who qualify for Part C services have a signed Individualized Family Service Program (IFSP) by no later than 6 months of age.

- $95 \%$ of children with acquired or late-identified hearing loss should have a signed IFSP within 45 days of diagnosis.

- 90\% of infants with confirmed hearing loss should receive a developmental assessment with standardized assessment protocols for language, speech, and nonverbal cognitive development by no later than 12 months of age. 
In developing quality enhancement programs, EHDI coordinators must engage all stakeholders so that there are both internal and external program monitors for every step. For example, hospitals may want to monitor the effectiveness of training screeners, referral rates, and the ability to address problems as they arise. Audiologists may want to monitor best methods of assessing hearing loss that result in the least number of office visits, monitor "no show" rates, and also address issues as they arise. The state EHDI program may want to monitor all birthing hospitals and look for outliers, referral practices, loss to follow-up, etc., and work with individual programs to improve outcomes.

\section{Analysis of Quality Enhancement Programs}

To ensure the best outcomes, JCIH and CDC recognize quality enhancement as being essential for EHDI systems. Thus, state EHDI programs must periodically evaluate how they are managing and meeting their objectives and identify the role and responsibility of the state EHDI coordinator. The EHDI coordinator is charged with developing partnerships with key stakeholders and community providers, establishing protocols and procedures for service delivery and monitoring, and implementing quality monitoring programs.

The purpose of this study was to complete a systematic review of quality improvement practices using a strengths, weaknesses, opportunities, and threats, or SWOT, and a threats, opportunities, weaknesses, and strengths, or TOWS, analysis to conduct an external review of EHDI program practices in quality enhancement programs, and to identify recommendations to enhance programming and overall outcomes.

\section{Methods}

Fifty-one EHDI coordinators were asked to complete an online survey. Related to quality improvement, the survey asked for the EHDI coordinator to report at least 1 item in the following four areas to assist with strategic planning: strength, weakness, opportunity, and threat. The responses obtained were reviewed by a panel of experts in the field and categorized into common themes in each of these four strategic planning areas. To generate recommendations from the SWOT analysis, a TOWS matrix was used to match identified strengths with opportunities (S-O strategy), strengths with threats (S-T strategy), weaknesses with opportunities (W-O strategy), and weaknesses with threats (W-T strategy). For an in-depth review of the methodology, the reader is referred to White and Blaiser, 2011.

\section{Results}

Forty-seven EHDI coordinators (92\%) completed the quality improvement area of the SWOT questionnaire. The respondents generated 66 items 
Table I. Frequency of themes within each SWOT category $(\mathrm{n}=218)$

\begin{tabular}{clc}
\hline SWOT Category & \multicolumn{1}{c}{ Themes } & Frequency n (\%) \\
\hline Strengths $(\mathrm{n}=66)$ & Evaluation Systems & $13(20 \%)$ \\
& Staffing & $11(17 \%)$ \\
& Data Application & $9(14 \%)$ \\
& Monitoring & $9(14 \%)$ \\
& Miscellaneous/No Comments & $7(11 \%)$ \\
& Follow-Up Strategies & $6(9 \%)$ \\
& Collaboration & $6(9 \%)$ \\
Weaknesses $(\mathrm{n}=49)$ & Planning & $5(8 \%)$ \\
& Staffing & $17(35 \%)$ \\
& Evaluation System & $7(14 \%)$ \\
& Collaboration & $5(10 \%)$ \\
& Data Quality & $4(8 \%)$ \\
& Data Sharing & $4(8 \%)$ \\
& Funding & $4(8 \%)$ \\
& Time Constraints & $4(8 \%)$ \\
& Miscellaneous & $3(6 \%)$ \\
& Data Security & $1(2 \%)$ \\
& Evaluation System & $14(27 \%)$ \\
& Collaborations & $12(23 \%)$ \\
& Data Systems & $8(15 \%)$ \\
& Miscellaneous/No Comments & $6(12 \%)$ \\
& Data Sharing/Evaluation System & $5(10 \%)$ \\
& Staffing & $4(8 \%)$ \\
& Funding & $3(6 \%)$ \\
& Funding Issues & $20(39 \%)$ \\
& Lack or Limited Staffing & $14(27 \%)$ \\
& Program Support & $8(16 \%)$ \\
& Miscellaneous/No Comments & $6(12 \%)$ \\
& & $3(6 \%)$ \\
& &
\end{tabular}

in the strength category, 49 items in the weaknesses category, 52 items in the opportunities category, and 51 items in the threats category for a total of 218 responses. Based on the responses, several specific themes were generated for each category: 8 for strengths, 9 for weaknesses, 7 for opportunities, and 5 for threats (Table 1). Each category also had a miscellaneous section that included no comment, N/A, or items that did not fit within the themes identified.

\section{Strength Characteristics}

The most frequently cited strengths were evaluation system (20\%), staffing (17\%), and data application and monitoring (tied, 14\%) (Table 2). Twenty percent of the respondents reported that they had an evaluation system integrated into their state EHDI program, ranging from patient satisfaction 
Table 2. Top three themes for strengths

\begin{tabular}{ll}
\hline \multicolumn{1}{c}{ Themes } & \multicolumn{1}{c}{ Descriptions } \\
\hline Evaluation Systems & Targeted quality assurance activities. \\
& Preparing and compiling surveys. \\
& Trend analysis on longitudinal data. \\
& Epidemiologist support. \\
Staffing & Staff dedicated to quality monitoring. \\
& Understanding of goals and objectives. \\
& Compliance reports are compiled and disseminated to \\
Monitoring & hospitals and providers. \\
& Ongoing, onsite inservice programs. \\
& Systems are in place for monitoring all sites involved \\
& with EHDI.
\end{tabular}

surveys to longitudinal data trend analysis. Seventeen percent of EHDI coordinators stated that engaging staff and service providers to understand the importance of quality monitoring was a significant strength, and some noted that having an epidemiologist on staff was extremely important in an effective quality enhancement program. Sharing data with key stakeholders and having a monitoring program with updated data management systems were also significant strengths reported by $28 \%$ of respondents in establishing a quality enhancement program. These data systems were described as being real time, longitudinal, automated, and capable of generating usable reports.

Other strengths included effective follow-up strategies with providers and agencies $(9 \%)$, ongoing collaboration with essential stakeholders, vital records, audiologists, speech-language pathologists, hospitals, and state agencies $(9 \%)$, and having dedicated planning time (8\%).

\section{Weakness Characteristics}

The most frequently cited weaknesses were staffing (35\%), evaluation systems $(14 \%)$, and collaboration (10\%) (Table 3). Thirty-five percent of states reported that their main weakness was having inadequate staff to implement and monitor a quality enhancement program. Specifically, they reported having vacancies, no epidemiologists, lack of expertise, shared positions and not having allotted time, hiring freezes, and lack of time. There were several respondents $(14 \%)$ that reported they either had no evaluation component in place or had no way to monitor or report on how hospitals or providers were performing. The lack of buy-in by key stakeholders, such as pediatric audiologists and hospital staff, was also cited as a weakness by $10 \%$ of respondents. 
Table 3. Top three themes for weaknesses

\begin{tabular}{ll}
\hline \multicolumn{1}{c}{ Themes } & \multicolumn{1}{c}{ Descriptions } \\
\hline Staffing & Limited staff time dedicated to this cause. \\
& Hiring freeze, minimally staffed. \\
& No epidemiologist. \\
& Only collect aggregated data - not patient/client \\
specific. & No data security system in place (no back-up \\
Data & system in place). \\
& Lack of sharing of data. \\
& No program evaluation system. \\
& Evaluation of program and systems have not been \\
Evaluation System & explored. \\
& No system in place to monitor data reporting to \\
& ensure quality data input. \\
\hline
\end{tabular}

The absence of quality data $(8 \%)$, the lack of data sharing $(8 \%)$, and the loss of data by not having a security back-up plan (2\%) also were cited as significant weaknesses to quality enhancement programs. Time constraints to implement quality monitoring/improvement projects was also identified by $8 \%$ of EHDI coordinators.

\section{Opportunities for EHDI Programs}

The most frequently cited opportunities were evaluation systems (27\%), collaboration (23\%), and data systems (15\%) (Table 4). Several respondents $(27 \%)$

Table 4. Top three themes for opportunities

\begin{tabular}{|c|c|}
\hline Themes & Descriptions \\
\hline Evaluation System & $\begin{array}{l}\text { Grants request information on how states evaluate their } \\
\text { systems. } \\
\text { Conducting surveys and focus groups with targeted } \\
\text { stakeholders (physicians, parents). } \\
\text { Created working groups to develop quality assurance } \\
\text { programs. }\end{array}$ \\
\hline Collaboration & $\begin{array}{l}\text { Advisory council with subcommittees. } \\
\text { Working with other state agencies. } \\
\text { Building teams with hospital, audiologists, physicians, } \\
\text { early interventionists, etc. }\end{array}$ \\
\hline Data Systems & $\begin{array}{l}\text { New systems to expedite tracking and reporting of data. } \\
\text { Elimination of redundant data entry. } \\
\text { New procedures for data management and monitoring. }\end{array}$ \\
\hline
\end{tabular}


reported that they have been conducting focus groups, surveys, and reviewing their programs to develop a quality monitoring program. Twenty-three percent of respondents noted that participating in the NICHQ Learning Collaborative helped develop and integrate quality efforts into their programs, and that having an advisory council or board with active subcommittees helped with overall collaboration. The implementation of reports and data sharing were also mentioned as opportunities by $15 \%$ of respondents. EHDI coordinators reported that having a new data management system helped them implement a quality enhancement program by eliminating unnecessary, redundant data entry.

Other opportunities included effective data sharing and evaluation systems $(10 \%)$, filling much needed staffing positions $(8 \%)$, and available funding $(6 \%)$.

\section{Threats to EHDI Programs}

The most frequently cited threats were funding (39\%), staffing $(27 \%)$, and program support (16\%) (Table 5). Overwhelmingly, 39\% of respondents reported that funding, budget cuts, and fiscal crises were all threats to their program. Twenty-seven percent of EHDI coordinators identified a lack of qualified staff members who understand how to analyze and interpret the data and who can develop meaningful reports to support system change as a threat. Furthermore, coordinators reported not having sufficient staff support due to turnover, cutbacks, or time constraints. Sixteen percent of EHDI coordinators mentioned that a lack of buy-in from key stakeholders, including audiologists, physicians, and state administrators, led to little collaboration. The other identified threat was the lack of time $(8 \%)$ to implement a quality enhancement program.

Table 5. Top three themes for threats

\begin{tabular}{ll}
\hline \multicolumn{1}{c}{ Themes } & \multicolumn{1}{c}{ Descriptions } \\
\hline Funding & Fiscal crisis. \\
& Budget cuts. \\
& Loss of funding. \\
& Not enough time to complete quality assurance projects. \\
Not enough staff or staff with limited knowledge of & data analysis. \\
& Staff turnover and cutbacks. \\
& Limited oversight. \\
& Administration not supportive of EHDI. \\
Program Support & Lack of buy-in by audiologists and physicians. \\
&
\end{tabular}




\section{TOWS Analysis Matrix}

By applying the TOWS analysis matrix to the themes identified in the SWOT analysis, specific recommendations, or "strategic options," were derived from the internal and external environmental factors identified by the EHDI coordinators. By systematically pairing the four categories of the SWOT analysis, recommendations can be derived to maximize resources and effect positive change. Based on the SWOT themes, 12 individual recommendations emerged (Table 6). To maximize opportunities, building strengths can maximize efficiency and lead to positive change (S-O strategy). For example, to have a quality enhancement program, state EHDI programs must have an effective and efficient data management system. To overcome weaknesses, opportunities must be pursued (W-O strategy). For example, with an engaged advisory council, coordinators can assist the EHDI program in developing a program evaluation system that can lead to overall improvements in the services provided. Identifying ways to use strengths will ultimately reduce vulnerability

Table 6. TOWS analysis matrix for quality enhancement

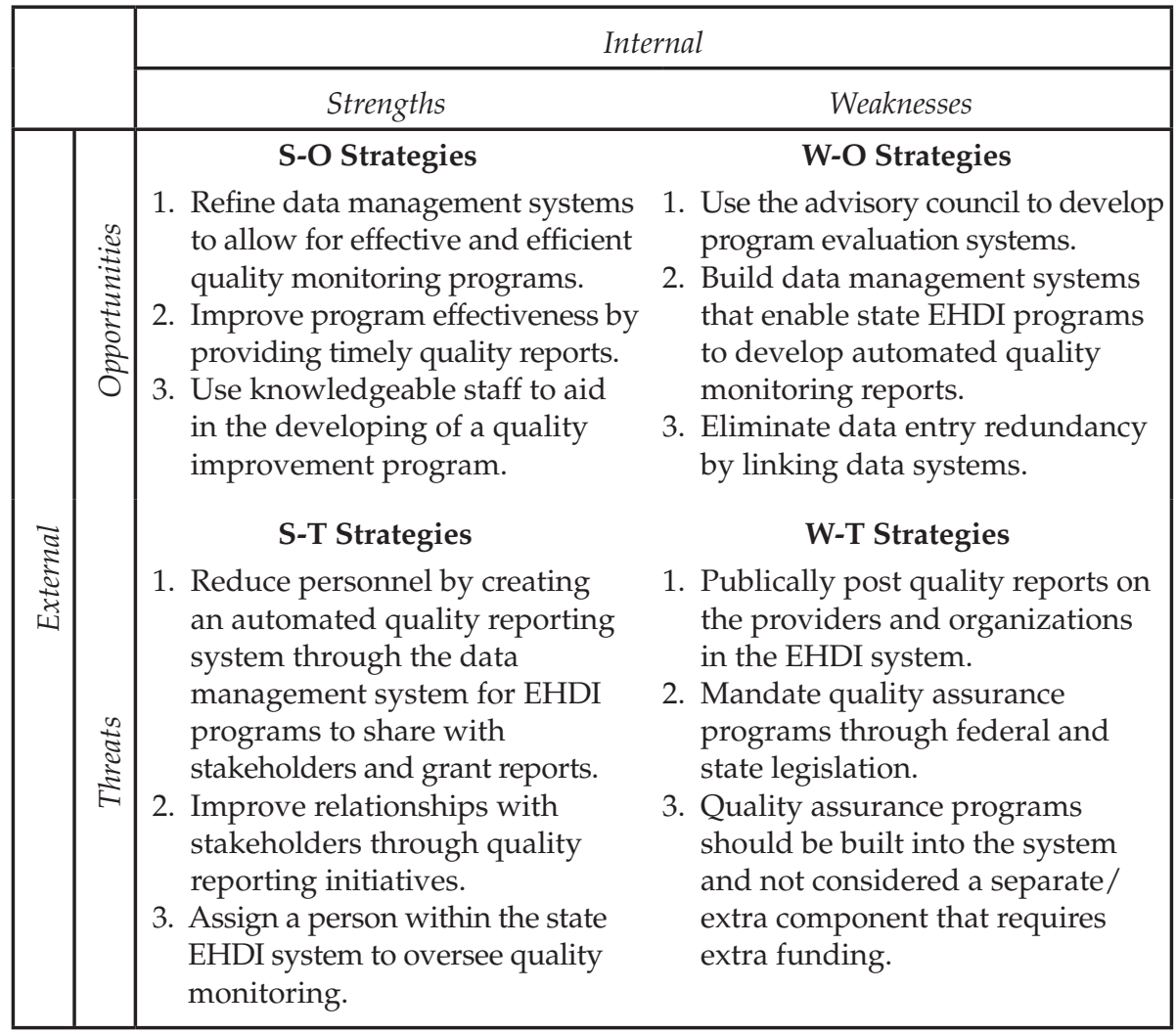

$\mathrm{S}=$ Strength $\mathrm{W}=$ Weakness $\mathrm{O}=$ Opportunity $\mathrm{T}=$ Threat 
to threats (S-T strategy). For example, creating an automated reporting system through the data management system can reduce the need for additional staff and ultimately reduce expenses. Finally, establishing defensive plans will help to prevent weaknesses from making programs and services susceptible to threats (W-T strategy). For example, to address "buy-in" from providers, key stakeholders, and families, publically post all quality reports so that everyone can see what is happening with the program. Out of these 12 recommendations, 3 strategies emerged to help state EHDI programs with quality enhancement programs. Specifically, programs should: 1) upgrade to comprehensive data management systems that will automatically generate meaningful reports based on the state benchmarks being measured; 2 ) integrate quality enhancement programs into the state system at all levels and not as a separate job; and 3) publically post all quality measures to hold the EHDI system accountable.

\section{Limitations}

Limitations of this study include those inherent in the application of SWOT/ TOWS analysis when applied to rapidly changing conditions. SWOT analysis is a subjective tool that explores conditions that are perceived rather than objectively measured. In this sense, the SWOT analysis is influenced by the perspectives of those who completed the surveys as well as those who analyzed the responses. Furthermore, SWOT analysis characterizes the situation at a specific point in time. In addition, SWOT analysis, as applied here, resulted in overlapping themes within a category. For example, staffing could be identified as a strength, weakness, opportunity, or threat (see White \& Blaiser, 2011, for additional information regarding the selection of SWOT/TOWS for this study and further comment regarding interpretation and application of findings).

\section{Summary}

EHDI coordinator responses in each of the SWOT areas provide insights to a number of current issues and what they view as critical elements. While some states reported having adequate staffing support, effective data management systems, and sufficient quality enhancement programs, other states reported these as weaknesses or threats. This is to be expected for a variety of reasons, such as 1) all EHDI programs did not start at the same time so some states have more experience than others; 2) funding levels, administrative support, and legislation vary state by state; and 3) each state has different population needs (i.e., rural vs. urban settings, languages spoken, number of providers available, etc.). Knowing what is reported to be helpful by states that have successfully implemented quality enhancement programs may help other states prioritize their needs and focus areas. Furthermore, the TOWS analysis matrix provides states with targeted items that are necessary across all states. Having effective data management tools is essential with any quality enhancement program. Integrating quality enhancement programs into the job descriptions of EHDI team members 
eliminates the need for another staff position and encourages acceptance from key individuals. Furthermore, posting state results should help educate people about EHDI, promote what is working well, identify areas needing improvement, and inform stakeholders of efforts underway to addresses those areas.

Based on this review, many states have some form of a quality enhancement program in place while others do not. The JCIH (2007) has clearly recommended the quality indicators that should be monitored by each state. Not only should these quality indicators be measured, but each step and system within EHDI should be systematically analyzed on a regular basis for continued quality improvement. Without ongoing assessment and analysis of defined outcomes, EHDI programs may find themselves operating under assumptions that over- or underestimate their actual performance.

\section{Acknowledgement}

The work reported in this article was funded in part by the Maternal and Child Health Bureau under Cooperative Agreement \# U52MC04391 with the National Center for Hearing Assessment and Management at Utah State University. The opinions expressed in the article are those of the authors and do not necessarily reflect those of the Bureau.

\section{References}

Centers for Disease Control and Prevention (CDC). (2010a). EHDI hearing screening and follow-up survey. Retrieved May 19, 2011, from http://www. cdc.gov/ncbddd/EHDI/data.htm.

Centers of Disease Control and Prevention (CDC). (2010b). Identifying infants with hearing loss - United States, 1999-2007. Morbidity and Mortality Weekly Report, 59(8), 220-223.

Finitzo, T., \& Gross, S. (2003). Quality monitoring for early hearing detection and intervention programs to optimize performance. Mental Retardation and Developmental Disabilities Research Reviews, 9, 73-78.

Joint Committee on Infant Hearing (JCIH). (2007). Year 2007 position statement: Principles and guidelines for early hearing detection and intervention programs. Pediatrics, 120(4), 898-921.

U.S. Department of Health and Human Services (DHHS). (2010). Healthy people 2020: Hearing and other sensory or communication disorders. Retrieved May 19, 2011, from http:/ / www.healthypeople.gov/2020/topicsobjectives2020/ overview.aspx?topicid=20.

White, K., \& Blaiser, K. (2011). Strategic planning to improve early hearing detection and intervention programs. The Volta Review, 111(2), 83-108.

White, K., Forsman, I., Eichwald, J., \& Muñoz, K. (2010). The evolution of early hearing detection and intervention programs in the United States. Seminars in Perinatology, 31(2), 170-189. 
The Volta Review, Volume 111(2), Summer 2011, 267-279

\title{
Interdisciplinary Collaboration in EHDI Programs
}

\author{
Lauri Nelson, Ph.D.; K. Todd Houston, Ph.D., CCC-SLP, LSLS Cert. AVT; \\ Jeff Hoffman, M.S., CCC-A; and Tamala S. Bradham, Ph.D., CCC-A
}

State coordinators of early hearing detection and intervention (EHDI) programs were asked to complete a strengths, weaknesses, opportunities, and threats, or SWOT, analysis that consisted of 12 evaluative areas of EHDI programs. For the interdisciplinary area, 47 coordinators responded with 224 items, and themes were identified within each SWOT category. A threats, opportunities, weaknesses, and strengths, or TOWS, analysis yielded 8 recommendations that focused on specific themes. Out of these 8 recommendations, 3 strategies emerged: 1) increase communication through state EHDI program advisory boards or through a coalition of stakeholders and professionals; 2) develop a mechanism of linking or sharing data to reduce communication breakdown between providers and the EHDI program; and 3) monitor all goals and objectives to hold providers/agencies accountable for their part within the EHDI system. Addressed appropriately, these recommendations could improve interdisciplinary collaborations in state EHDI programs.

\section{Introduction}

By definition, collaboration is a process where two or more people or organizations work jointly with others or together, especially in an intellectual endeavor (Merriam-Webster Dictionary, 2011). More specifically, Bronstein (2003) describes interdisciplinary collaboration as "an effective interpersonal process that facilitates the achievement of goals that cannot be reached when

Lauri Nelson, Ph.D., is an Assistant Professor in the Department of Communicative Disorders and Deaf Education at Utah State University. K. Todd Houston, Ph.D., CCC-SLP, LSLS Cert. $A V T$, was an Assistant Professor in the Department of Communicative Disorders and Deaf Education at Utah State University at the time of this study and is currently at The University of Akron. Jeff Hoffman, M.S., CCC-A, is a Senior Research Scientist and Audiologist in the National Center for Hearing Assessment and Management at Utah State University. Tamala S. Bradham, Ph.D., CCC-A, is an Assistant Professor in the School of Medicine, Department of Hearing and Speech Sciences, at Vanderbilt University. Correspondence concerning this manuscript may be directed to Dr. Nelson at lauri.nelson@usu.edu. 
individual professionals act on their own" (p. 299). The early hearing detection and intervention (EHDI) program encompasses a broad continuum of services from several agencies and service providers with the goal of ensuring that all infants and toddlers with hearing loss are identified as early as possible and provided with timely and appropriate medical, audiological, and educational intervention (National Center for Hearing Assessment and Management [NCHAM], 2010). Stakeholders in EHDI programs include parents and families along with a range of professionals, such as EHDI coordinators, early interventionists, physicians, audiologists, state department personnel, and educational personnel. There are multiple components to EHDI programs, such as the initial newborn hearing screening, systems for referral and followup upon failed screening, medical evaluation, referral to medical specialists as individually appropriate, diagnostic audiological testing, ongoing audiological management, family-centered support throughout the diagnostic process, and family-centered early intervention services utilizing developmentally appropriate practices in the family's chosen mode of communication. The complexities of the EHDI system and the large number of professionals and agencies involved illustrate the need for a system of interagency collaboration to develop, implement, and maintain an effective and comprehensive statewide EHDI program. Although it is a likely assumption that all stakeholders associated with newborn hearing screening have the common goal of providing excellent services to infants and their families, the notion of what "collaboration" means, determining the composition of excellent services, finding the intersection of common goals among all service providers, or having all stakeholders agree on the most appropriate avenue to reach those goals remains a challenge for EHDI programs.

The percentage of newborns in the United States screened for hearing has increased dramatically over the past decade, from 3\% in 1995 to $97 \%$ in 2007 (CDC, 2010). In those states where newborn hearing screening programs have been effectively implemented, the age at which infants are identified with hearing loss has been reduced to an average age of 2-3 months (White, Forsman, Eichwald, \& Muñoz, 2010). This positive trend results in identifying more infants and young children who need services. State, medical, and educational agencies must continually deal with the ever-growing demands of providing more services with fewer resources. As a result, service providers frequently struggle to fulfill essential job requirements with little time for outside communications or collaborations. Unfortunately, this trend perpetuates the cycle of status-quo services and stalls forward momentum necessary to elevate programs to increased collaboration for excellence in service delivery.

An evaluation of research investigating factors related to successful or unsuccessful collaborative partnerships indicates the following factors as the primary contributors to collaboration barriers: a) lack of effective communication, b) lack of time, c) unclear goals and objectives, d) gaps between screening and diagnostic services, e) different definitions of collaboration, f) resistance 
to change, and g) insufficient understanding of other agencies' policies or priorities (Harbin, 1996; Johnson, Zorn, Kai Yung Tam, Lamontagne, \& Johnson, 2003; Stegelin \& Jones, 1991). Solutions for improved collaboration by EHDI programs are multidimensional and require more than a broad stroke across all agencies. Each state faces its own set of unique circumstances and challenges, with solutions that require state-specific implementation. However, in an evaluation of interagency collaboration, Johnson et al. (2003) identified seven key factors essential for a successful interagency partnership. They are:

1) Commitment: If agencies, or specific members within the agency, do not have a commitment to the collaboration, it likely will fail. There must be a high level of trust with sharing of goals and program vision.

2) Communication: This is an essential and key component to effective collaboration. While obvious in theory, implementing a specific and effective communication plan between agencies is frequently overlooked. A proactive approach with frequent and pre-arranged opportunities to communicate increases the likelihood of a collaborative partnership.

3) Strong Leadership from Key Decision Makers: Team involvement should include someone from each agency who understands the agencies' priorities and who has the authority to make decisions.

4) Understand the Culture of the Agency: Collaborating agencies should recognize that each organization has its own culture (e.g., rules, values, communication style), and taking the time to understand potential differences will improve the collaborative partnership.

5) Provide Adequate Resources: If the collaborative partnership is important, then time and resources must be allocated for a successful partnership to be achieved.

6) Minimize Turf Issues: Turf issues are likely to occur and should not be ignored or go unacknowledged. Agencies should anticipate their appearance and develop a plan for addressing issues as they occur.

7) Engage in Preplanning: Collaborating agencies should clearly articulate the partnership goals and the anticipated outcomes of the collaboration.

Collaboration among stakeholders who provide services to infants and toddlers with disabilities is not just a good idea. The Individuals with Disabilities Education Act (IDEA) requires the development of a "statewide, comprehensive, coordinated, multidisciplinary, interagency system" of services (20 U.S.C. $\$ 631(b))$. Under Part C, the infants and toddlers with disabilities program, and Part B, section 619 or the preschool program, IDEA governs interagency coordination for young children with disabilities, from birth to 5 years of age, and their families. To accomplish this, each state is required to establish an Interagency Coordinating Council (ICC) for the purpose of assuring that needed services are appropriate and available to all eligible infants and young children and their families (National Early Childhood Technical Assistance 
Center, 2010). The ICC is comprised of governor-appointed members including both professionals and parents who represent families of young children with disabilities, and it should be an active component to statewide, familycentered early intervention services. It is essential that states demonstrate effective implementation of service coordination across agency lines to provide the array of necessary services for infants and young children with disabilities and their families. Interagency agreements, including written policies, procedures, and guidelines, are required to be in place to facilitate communication and coordination across agencies and at the state and local levels.

Identification of the common challenges faced by EHDI programs related to interdisciplinary collaboration of services for young children with hearing loss can provide valuable information to improve program effectiveness and lead to better outcomes for infants who do not pass the newborn hearing screening. The purpose of this study was to provide a systematic review of interagency collaboration using a strengths, weaknesses, opportunities, and threats, or SWOT, and a threats, opportunities, weaknesses, and strengths, or TOWS, analysis to determine current EHDI program practices in interdisciplinary collaboration, and to identify factors that contribute to program effectiveness and lead to better outcomes for infants who participate in EHDI programs.

\section{Methods}

Fifty-one EHDI coordinators were asked to complete an online survey. Under interdisciplinary collaboration, the survey asked the EHDI coordinator to report at least 1 item in the following four areas to assist with strategic planning: strength, weakness, opportunity, and threat. The responses obtained were reviewed by a panel of experts in the field and categorized into common themes in each of these four strategic planning areas. To generate recommendations from the SWOT analysis, a TOWS matrix was used to match identified strengths with opportunities (S-O strategy), strengths with threats (S-T strategy), weaknesses with opportunities (W-O strategy), and weaknesses with threats (W-T strategy). For an in-depth review of the methodology, the reader is referred to White and Blaiser, 2011.

\section{Results}

Forty-seven EHDI coordinators (92\%) completed the interdisciplinary collaboration section of the SWOT questionnaire. Respondents generated 66 items in the strength category, 52 items in the weakness category, 51 items in the opportunities category, and 55 items in the threats category for a total of 224 responses (Table 1). Based on these responses, several specific themes were generated for each category: 6 for strengths, 6 for weaknesses, 5 for opportunities, and 6 for threats. Each category also had a miscellaneous 
Table I. Frequency of themes within each SWOT category $(n=224)$

\begin{tabular}{clc}
\hline SWOT category & \multicolumn{1}{c}{ Themes } & Frequency $n(\%)$ \\
\hline Strengths (n=66) & Systems Integration and Linkage & $23(35 \%)$ \\
& Positive Professional Relationships & $20(30 \%)$ \\
& Establishment of Advisory Boards & $10(15 \%)$ \\
& Working Together on Specific Projects & $6(9 \%)$ \\
& Miscellaneous/No Comments & $4(6 \%)$ \\
& Legal Agreements or Contracts & $3(5 \%)$ \\
Weaknesses $(\mathrm{n}=52)$ & Poor Professional Relationships & $18(35 \%)$ \\
& Breakdowns in Sharing/Reporting Data & $10(19 \%)$ \\
& Program Infrastructure & $9(17 \%)$ \\
& Insufficient Education and Training & $7(13 \%)$ \\
& Poor Coordination of Services & $7(13 \%)$ \\
& Miscellaneous/No Comments & $1(2 \%)$ \\
Opportunities $(\mathrm{n}=51)$ & Improved Interagency Relationships & $14(27 \%)$ \\
& Improved Systems Integration and Data & $14(27 \%)$ \\
& Sharing & $11(22 \%)$ \\
& Development of Stakeholder Groups / & \\
& Committees & $9(18 \%)$ \\
& Improved Training and Service Provision & $3(6 \%)$ \\
& Miscellaneous/No Comments & $16(29 \%)$ \\
& Funding and Reimbursement & $12(22 \%)$ \\
& Barriers/Roadblocks & $11(20 \%)$ \\
& Miscellaneous/No comments & $4(7 \%)$ \\
& Time Constraints & $3(5 \%)$ \\
\hline & Poor Systems Integration &
\end{tabular}

section, which included no comment, N/A, or items that did not fit within the themes identified.

\section{Strength Characteristics}

The most frequently cited strengths were system integration and linkage (35\%), positive professional relationships (30\%), and establishment of an advisory board (15\%) (Table 2). Having an integrated system of communication linking EHDI services with other stakeholders was cited by 35\% of respondents as positively contributing to interagency collaboration. EHDI coordinators specifically cited the value of having electronic data tracking software connecting newborn hearing screening results between key state agencies (e.g., metabolic screening programs, Part C, vital statistics). Respondents that have implemented an electronic mechanism for sharing data reported fewer communication breakdowns, decreased loss to follow-up rates, and better facilitation of timely referrals to appropriate agencies. 
Table 2. Top three themes for strengths

\begin{tabular}{cl}
\hline \multicolumn{1}{c}{ Themes } & \multicolumn{1}{c}{ Description } \\
\hline $\begin{array}{c}\text { Systems Integration } \\
\text { and Linkage }\end{array}$ & $\begin{array}{c}\text { EHDI is housed in the appropriate agency to allow for } \\
\text { strong collaboration. } \\
\text { Work closely with hospitals, universities, early } \\
\text { intervention programs, professionals, and other agencies. }\end{array}$ \\
$\begin{array}{c}\text { Established relationships with key stakeholders. } \\
\text { Positive Professional } \\
\text { Relationships }\end{array}$ & $\begin{array}{l}\text { A small state allows you to know everyone. } \\
\text { Positive attitudes. }\end{array}$ \\
Establishment of & Longstanding active board. \\
Advisory Boards & Diverse board. \\
& Mandated by legislation. \\
\hline
\end{tabular}

The development or strengthening of professional relationships was identified by $30 \%$ of EHDI coordinators as a primary factor that positively contributed to interagency collaboration. Respondents described the value of taking the time to get to know various stakeholders by name, fostering opportunities to get to know "individuals" rather than "agencies," working together on shared projects, and establishing multiagency continuing education or training opportunities.

Several respondents $(15 \%)$ specifically described the importance of having a state advisory committee or taskforce to foster interdisciplinary collaboration among all the various stakeholders. An active and diverse advisory board, with participants from both the public and private sectors, can facilitate communication among agencies, can assist legislative or systemic program improvements, and can facilitate state connections with family support agencies. Similarly, establishment of, or participation in, a state taskforce or Part C council can increase partnership visibility, provide opportunities to identify program needs, and foster interpersonal connections.

Other strengths identified included working together on projects $(9 \%)$ and legal agreements and/or contracts (5\%). Receiving assistance for the NICHQ Learning Collaborative project was mentioned as a strength along with both private and public sectors working together for a common good. Have agreements or contracts in place also facilitated healthy collaborations.

\section{Weakness Characteristics}

The most frequently cited weaknesses were poor professional relationships (35\%), breakdown in sharing/reporting data (19\%), and program infrastructure (17\%) (Table 3). Thirty-five percent of EHDI coordinators described difficult interagency relationships as the most significant program weakness 
Table 3. Top three themes for weaknesses

\begin{tabular}{cl}
\hline \multicolumn{1}{c}{ Themes } & \multicolumn{1}{c}{ Description } \\
\hline Poor Professional Relationships & $\begin{array}{l}\text { Difficult to find times to meet. } \\
\text { Difficulties getting key stakeholders "to the table." } \\
\text { Old history/baggage. }\end{array}$ \\
Breakdowns in Sharing/ & Lack of database. \\
Reporting Data & Restrictions in sharing data between agencies and \\
providers (i.e., privacy laws). & Amount of time it takes to get data. \\
Program Infrastructure & EHDI and early intervention (Part C) housed in \\
& difference departments. \\
& EHDI is not mandated. \\
& Changing in personnel/Advisory board \\
members - always playing "catch-up."
\end{tabular}

resulting in barriers to collaboration. Respondents described some stakeholders as having little or no "buy in" to full participation in the EHDI process, resulting in poor communication and coordination of information. Several EHDI coordinators reported that turf issues contributed to collaboration barriers, with several respondents indicating that "old history" and lack of trust between colleagues had a contaminating impact on professional relationships. In addition, fewer face-to-face meetings and insufficient time for connecting with colleagues from other agencies contributed further to poor communication and collaboration.

The inability to connect electronically for data sharing was a reported collaboration barrier for $19 \%$ of EHDI coordinators. These coordinators described breakdowns in the diagnostic testing process and with making and receiving appropriate referrals due to poor communication. They reported that having a web-based or electronic database connection with either the state metabolic screening program, immunizations program, or the vital statistics/birth certificate registry would minimize these occurrences.

Poor program infrastructure was identified as a barrier to collaboration by $17 \%$ of EHDI coordinators. Staff shortages and a high staff turnover rate contributed to the difficulty of establishing and maintaining strong interagency relationships. Poor infrastructure across all key EHDI agencies, and thus a lack of program leadership, resulted in minimal service coordination or collaboration particularly when physically housed in separate locations.

Additionally, EHDI coordinators reported insufficient education and training $(13 \%)$ and poor coordination of services $(13 \%)$ as weaknesses to their programs. 


\section{Opportunities for EHDI Programs}

The most frequently cited opportunities were improved interagency relationships (27\%), improved systems integration and data sharing $(27 \%)$, and development of stakeholder groups/committees (22\%) (Table 4). The predominant opportunities identified by EHDI coordinators for improving collaborative partnerships in EHDI services was improved interagency relationships and the establishment of an electronic database for tracking diagnostic results and interagency referrals. More than half of the EHDI coordinators $(54 \%)$ identified these two elements as key opportunities for successful EHDI services.

Twenty-two percent of EHDI coordinators identified the development and implementation of an advisory board as an opportunity that would improve interagency collaboration and service delivery. Other opportunities included the need for increased opportunities for education and training to improve EHDI interagency collaboration outcomes (18\%), including attending conferences, providing parent training, and using NCHAM training materials.

\section{Threats to EHDI Programs}

The most frequently cited threats were funding and reimbursement $(29 \%)$, barriers / roadblocks (22\%), and time constraints (16\%) (Table 5). Given the U.S. economic climate in 2010 it is not surprising that $29 \%$ of respondents noted a lack of funding as a threat to improved interagency collaboration. EHDI coordinators stated: "collaborations diminish when contractual relationships cease due to funding restrictions" and "everyone is protective of funding, and

Table 4. Top three themes for opportunities

\begin{tabular}{ll}
\hline \multicolumn{1}{c}{ Themes } & Description \\
\hline $\begin{array}{l}\text { Improved Interagency } \\
\text { Relationships }\end{array}$ & $\begin{array}{c}\text { Building better rapport with other agencies and } \\
\text { providers. } \\
\text { Willingness of agency administration to meet and } \\
\text { discuss EHDI. }\end{array}$ \\
$\begin{array}{c}\text { Improved Systems Integration } \\
\text { and Data Sharing }\end{array}$ & $\begin{array}{c}\text { Creating agreements between agencies and } \\
\text { providers. } \\
\text { National EHDI conference allows for networking } \\
\text { and learning opportunities. } \\
\text { Pooling resources and finding alternative partners } \\
\text { for needed services. }\end{array}$ \\
$\begin{array}{c}\text { Forming workgroups and committees. } \\
\text { Binging on new members to the advisory board. } \\
\text { Groups/Committees }\end{array}$ & Bring \\
\hline
\end{tabular}


Table 5. Top three themes for threats

\begin{tabular}{|c|c|}
\hline Themes & Description \\
\hline Funding and Reimbursement & $\begin{array}{l}\text { Budget cuts. } \\
\text { Limited resources. }\end{array}$ \\
\hline Barriers/Roadblocks & $\begin{array}{l}\text { Competing priorities that may jeopardize } \\
\text { collaborative relationships. } \\
\text { Lack of qualified providers. } \\
\text { Building relationships is difficult due to turnover in } \\
\text { legislators, agency administration, staff, advisory } \\
\text { board members, providers, etc. }\end{array}$ \\
\hline Time Constraints & $\begin{array}{l}\text { Lack of time. } \\
\text { Collaboration takes time and resources, which are } \\
\text { limited. }\end{array}$ \\
\hline
\end{tabular}

support dwindles when projects require shared staff or shared funding from a partner agency." Effectively addressing the funding dilemma will require energy and commitment for state-specific financial solutions. Accomplishing this is directly connected to the second most frequently cited threat to interagency collaboration.

Twenty-two percent of respondents reported that system barriers/roadblocks and a lack of commitment or desire for strong interagency collaboration were threats to their program's collaborative efforts. Partnering agencies who have unresolved disputes, unclear goals, and lack of commitment are prime for failed partnerships. Confounding these dilemmas are the realities of time constraints, as reported by $16 \%$ of EHDI coordinators. The threats to establishing and maintaining positive interagency collaborations are significant, and state agencies are urged to complete their own state-specific evaluations to determine proactive goals for successful interagency collaborations now and in the future.

Other threats included poor systems integration $(7 \%)$, such as poor understanding, lack of outpatient providers cooperating with EHDI, and lack of integration of data systems, and federal privacy requirements $(5 \%)$.

\section{TOWS Analysis Matrix}

By applying the TOWS analysis matrix to the themes identified in the SWOT analysis, specific recommendations, or "strategic options," were derived from the internal and external environmental factors identified by the EHDI coordinators. By systematically pairing the four categories of the SWOT analysis, recommendations can be derived to maximize resources and effect positive change. Based on the SWOT themes, 8 individual recommendations emerged (Table 6). To maximize opportunities, the identification of program strengths 
Table 6. TOWS analysis matrix for interdisciplinary collaboration

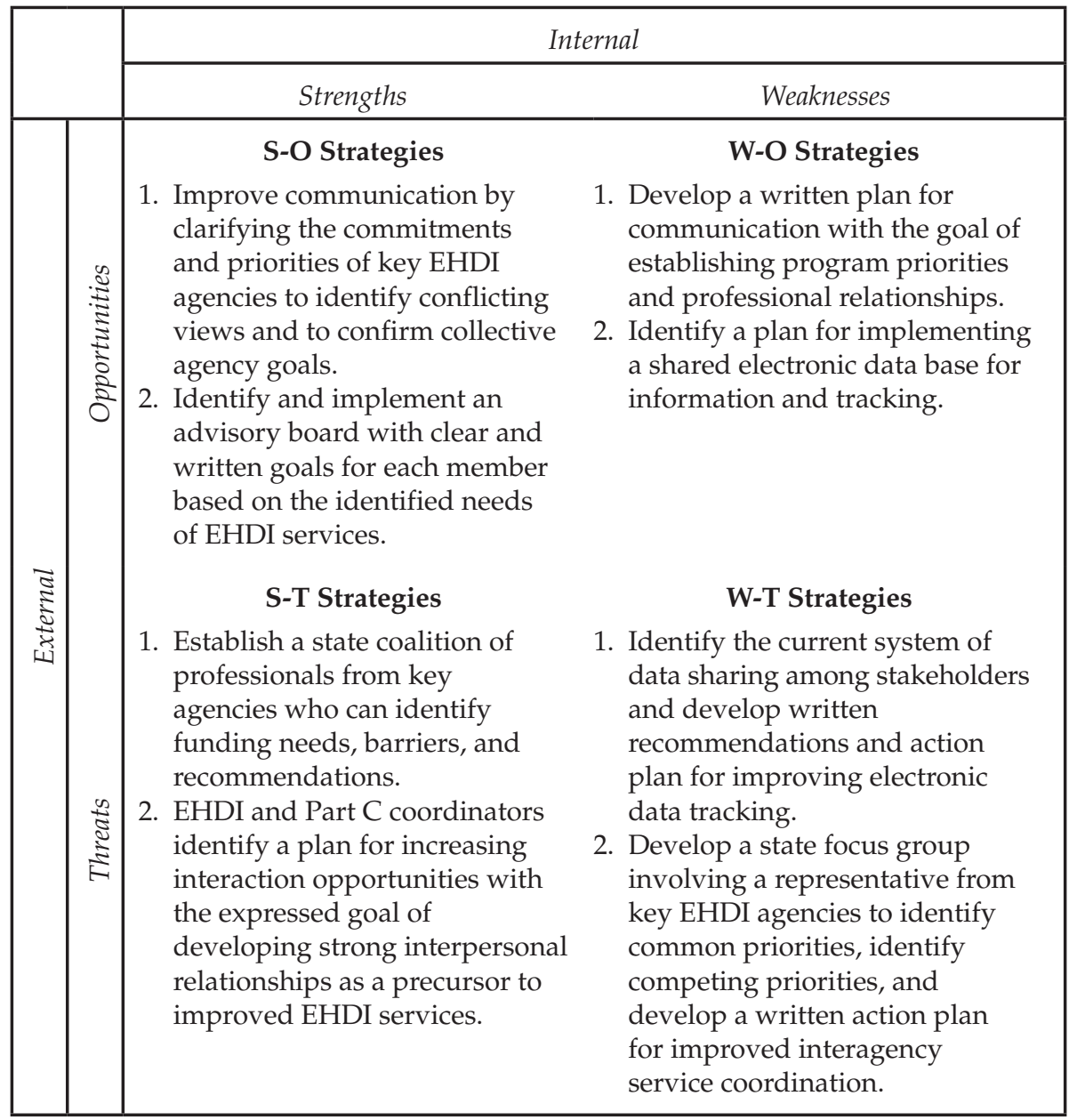

$\mathrm{S}=$ Strength $\mathrm{W}=$ Weakness $\mathrm{O}=$ Opportunity $\mathrm{T}=$ Threat

can lead to positive change (S-O strategy). For example, communication can be increased by clarifying the commitments and priorities of key EHDI service providers, resulting in an opportunity for state EHDI programs to better identify conflicting views, confirm the goals of each agency, and develop an action plan that acknowledges potential barriers for meeting the agreed upon EHDI goals and objectives. By pursuing opportunities, weaknesses can be minimized (W-O strategy). For example, identifying a plan for implementing a shared electronic database for tracking and referrals can reduce communication breakdowns between service providers, resulting in an opportunity to improve coordination of services statewide across several EHDI agencies. 
Identification of ways to use strengths can ultimately reduce vulnerability to threats (S-T strategy). For example, a state coalition of professionals from key agencies can identify barriers to providing effective services and make recommendations for positive change prior to issues becoming a significant threat to EHDI services. Finally, establishing defensive plans will help to prevent weaknesses from making programs susceptible to threats (W-T strategy). For example, the development of a proactive focus group to identify weaknesses within a state EHDI system and of a written action plan to address state needs for improving services can ultimately minimize the program weaknesses, therefore minimizing threats to the EHDI system. From the 8 recommendations, 3 strategies emerged for improving interdisciplinary collaboration. Specifically, programs should: 1) increase communication through their advisory board or coalition group of key stakeholders and professionals; 2) develop a mechanism of linking or sharing data to reduce communication breakdown between providers and the EHDI program; and 3) monitor all goals and objectives and hold providers/agencies accountable for their part within the EHDI system.

\section{Limitations}

Limitations of this study include those inherent in the application of SWOT / TOWS analysis when applied to rapidly changing conditions. SWOT analysis is a subjective tool that explores conditions that are perceived rather than objectively measured. In this sense, the SWOT analysis is influenced by the perspectives of those who completed the surveys as well as those who analyzed the responses. Furthermore, SWOT analysis characterizes the situation at a specific point in time. In addition, SWOT analysis, as applied here, resulted in overlapping themes within a category. For example, relationships could be identified as a strength and a weakness (see White \& Blaiser, 2011, for additional information regarding the selection of SWOT/TOWS for this study and further comment regarding interpretation and application of findings).

\section{Summary}

Those involved in state EDHI systems recognize that early identification, appropriate medical and audiological management, family support, and family-centered early intervention services require the coordination and collaboration of many different professionals, organizations, and agencies. Interdisciplinary collaboration is recognized as an essential component to providing quality health and educational services to children and families (Alberg, Wilson, \& Roush, 2006; Mitchell \& Crittensen, 2000; Petri, 2010). However, while the concept is not new, the implementation of effective interdisciplinary collaboration into standard practice is challenging. 
The results of the SWOT analysis reiterated and confirmed the findings and recommendations of Johnson et al. (2003). As identified by EHDI coordinators, there must be a commitment to the collaborative partnership. Unless there is a shared priority, relationships will not be strengthened nor will time be allocated to meet the collaborative partnership needs. Seemingly minor gestures (e.g., learning the names of EHDI colleagues, extending invitations to attend local training opportunities) can strengthen collaborative partnerships and could potentially set the stage for developing meaningful dialogue in which program obstacles can be addressed and cooperative plans for growth and development can occur across the EHDI continuum of services. Proactively identifying opportunities for direct interaction, shared education and training, and the explicit identification of shared barriers can lay the foundation for growth.

There was overwhelming support for the importance of establishing an electronic data tracking system to connect key EHDI agencies. State EHDI agencies that do not have this capability continue to struggle with unwieldy communication requirements due to the number of state agencies involved in the diagnostic process and in early intervention services. Without an electronic data connection, too many infants are lost to follow-up and stakeholders are frustrated by the resulting breakdowns in service delivery. An electronic link would improve data tracking across agencies, improve communications regarding referrals and results status, and reduce turf issues that can impact continuity of service delivery. The sharing of electronic information is not a trivial barrier given the need to protect the privacy rights of the family. However, several agencies have found state-specific mechanisms for establishing electronic connections without violating privacy issues. When this is accomplished, it becomes a major contribution toward successful interagency collaboration, as illustrated by the EHDI respondents who reported that having a shared electronic data tracking system was a primary component to positive interagency collaboration.

Strong leadership and a shared commitment among key EHDI agencies to identify state-specific barriers and to develop a written action plan for improvement will increase effective interagency collaboration opportunities. Although the process can be challenging, the benefits of a cohesive and comprehensive service delivery model for children with hearing loss and their families can be substantial.

\section{Acknowledgement}

The work reported in this article was funded in part by the Maternal and Child Health Bureau under Cooperative Agreement \# U52MC04391 with the National Center for Hearing Assessment and Management at Utah State University. The opinions expressed in the article are those of the authors and do not necessarily reflect those of the Bureau. 


\section{References}

Alberg, J., Wilson, K., \& Roush, J. (2006). Statewide collaboration in the delivery of EHDI services. The Volta Review, 106(3), 259-274.

Bronstein, L. R. (2003). A model for interdisciplinary collaboration. Social Work, 48(3), 297-306.

Centers for Disease Control and Prevention (CDC). (2010). Annual data: Early hearing detection and intervention (EHDI) program. Retrieved May 24, 2011, from http://www.cdc.gov/ncbddd/hearingloss/ehdi-data.html.

Harbin, G. L. (1996). The challenge of coordination. Infants and Young Children, $8(3), 68-76$.

Johnson, J. J., Zorn, D., Kai Yung Tam, B., Lamontagne, M., \& Johnson, S. A. (2003). Stakeholders? Views of factors that impact successful interagency collaboration. Exceptional Children, 69(2), 195-209.

Merriam-Webster Dictionary. (2011). Collaboration. Retrieved May 19, 2011, from http://www.merriam-webster.com/dictionary/collaboration.

Mitchell, P. H., \& Crittensen, R. A. (2000). Interdisciplinary collaboration: Old ideas with new urgency. Washington Public Health, Fall Issue, 1-3.

National Center for Hearing Assessment and Management (NCHAM). (2010). National resource center for early hearing detection and intervention. Retrieved June 14, 2011, from http:/ / www.infanthearing.org/tas/index.html.

National Early Childhood Technical Assistance Center. (2010). Interagency coordination. Retrieved June 14, 2011, from http:/ /www.nectac.org/topics/ intercoord/intercoord.asp.

Petri, L. (2010). Concept analysis of interdisciplinary collaboration. Nursing Forum, 45(2), 73-82.

Stegelin, D. A., \& Jones, S. D. (1991). Components of early childhood interagency collaboration: Results of a statewide study. Early Education and Development, 2(1), 54-67.

White, K., \& Blaiser, K. (2011). Strategic planning to improve early hearing detection and intervention programs. The Volta Review, 111(2), 83-108.

White, K. R., Forsman, I., Eichwald, J., \& Muñoz, K. (2010). The evolution of early hearing detection and intervention programs in the United States. Seminars in Perinatology, 34(2), 170-179. 

The Volta Review, Volume 111(2), Summer 2011, 281-288

\title{
Future Directions for EHDI Programs: Recommendations from a Strategic Analysis
}

\author{
Tamala S. Bradham, Ph.D., CCC-A; and \\ K. Todd Houston, Ph.D., CCC-SLP, LSLS Cert. AVT
}

Over the past 20 years, tremendous strides have been made in establishing and strengthening early hearing detection and intervention (EHDI) programs in all 50 states and the U.S. territories. Approximately $97 \%$ of all newborns are screened for hearing loss prior to discharge from a birthing center or hospital (Centers for Disease Control and Prevention, 2010; White, Forsman, Eichwald, \& Muñoz, 2010). This is indeed a significant achievement. Unfortunately, nearly $60 \%$ of infants referred from newborn hearing screening do not receive a timely diagnosis (Russ, White, Dougherty, \& Forsman, 2010) and many are lost to follow-up. While approximately $77 \%$ of children confirmed to have a permanent hearing loss enroll in early intervention by the age of 6 months, not all of these children have access to the services they need for speech and language development (Russ, et al., 2010). Although EHDI programs have become more robust, considerable work remains to ensure that all children who do not pass a hearing screening receive the appropriate follow-up services regardless of where they live and regardless of whether they are insured privately or through Medicaid.

Complicating this situation is the paucity of data available to policy makers, researchers, administrators, and other stakeholders regarding the current status of EHDI programs in the United States. To address that need, in 2009 the National Center for Hearing Assessment and Management (NCHAM) at Utah State University embarked on a systemic review aimed at obtaining a clearer, operational picture of state EHDI programs. To conduct this review,

Tamala S. Bradham, Ph.D., CCC-A, is an Assistant Professor at the Vanderbilt Bill Wilkerson Center, Department of Hearing and Speech Sciences, at Vanderbilt University. K. Todd Houston, Ph.D., was an Assistant Professor in the Department of Communicative Disorders and Deaf Education at Utah State University at the time of this study and is currently at The University of Akron. Correspondence concerning this manuscript should be addressed to Dr. Bradham at tamala.bradham@vanderbilt.edu. 
12 major areas within the EHDI system were defined (Table 1) and coordinators of all state-based EHDI programs were asked to complete a strengths, weaknesses, opportunities, and threats, or SWOT, analysis (Ghazinoory, Abdi, \& Azadegan-Mehr, 2011). Results of the SWOT analysis were evaluated using a threats, opportunities, weaknesses, and strengths, or TOWS, matrix (Weihrich, $1982,1998)$ to generate recommendations that would enhance programming and overall outcomes for current EHDI practices.

Fifty-one EHDI coordinators were asked to complete an online survey on the 12 areas within the EHDI system. The survey asked the EHDI coordinator to report at least 1 strength, weakness, opportunity, and threat for use in strategic planning. The responses obtained were reviewed by a panel of experts in the field and categorized into common themes in each of the four strategic planning areas. To generate recommendations from the SWOT analysis, a TOWS matrix was used to match identified strengths with opportunities (S-O strategy), strengths with threats (S-T strategy), weaknesses with opportunities (W-O strategy), and weaknesses with threats (W-T strategy). For additional information regarding the SWOT/TOWS analysis and its use in the current study, the reader is referred to White and Blaiser, 2011.

The primary goal of the analysis was to identify internal and external factors that contribute to the success of an EHDI program, but also to identify obstacles confronted by EHDI programs. By identifying processes that are successful, resources can be optimally allocated in ways that improve overall quality and reduce duplication of effort. Conversely, by identifying potential barriers, solutions can be formulated.

The strengths of this SWOT analysis include a high response rate, a large amount of data, uniformity of respondents (i.e., state EHDI coordinators), and cross-discipline analysis and interpretation of the data. The response rates ranged from $92 \%$ to $98 \%$ for each of the 12 areas surveyed. There were 3,101 total responses with 907 responses for strengths, 788 responses for weaknesses, 694 responses for opportunities, and 712 responses for threats. Because state EHDI coordinators are responsible for all 12 areas evaluated, they were considered ideal respondents for this project.

To evaluate the findings, a heterogeneous group was essential to ensure that the data were appropriately interpreted and categorized. Thus, teachers of the deaf and hard of hearing, audiologists, speech-language pathologists, EHDI coordinators, and an adult with a congenital hearing loss analyzed each of the individual responses from the survey and reached consensus on how responses would be categorized.

Limitations of this study include those inherent in the application of a SWOT/TOWS analysis when applied to rapidly changing conditions. First, SWOT analysis is a subjective tool that explores conditions that are perceived rather than objectively measured. In this case, the SWOT analysis is dependent on the perspectives of those who completed the survey as well as those who analyzed the responses. Second, the SWOT analysis characterizes the situation 
Table I. The 12 EHDI components included in the SWOT analysis

\begin{tabular}{|c|c|}
\hline EHDI Area & Description \\
\hline $\begin{array}{l}\text { 1. Newborn Hearing } \\
\text { Screening }\end{array}$ & $\begin{array}{l}\text { This EHDI area covers aspects of newborn hearing } \\
\text { screening, including hospital screenings as well as } \\
\text { home births. }\end{array}$ \\
\hline $\begin{array}{l}\text { 2. Audiological } \\
\text { Evaluation }\end{array}$ & $\begin{array}{l}\text { This EHDI area covers important aspects of } \\
\text { audiological evaluation and documentation. }\end{array}$ \\
\hline 3. Early Intervention & $\begin{array}{l}\text { This EHDI area covers key aspects of early intervention } \\
\text { including, but not limited to, Part C, non Part-C, } \\
\text { communication options, and technology options. }\end{array}$ \\
\hline $\begin{array}{l}\text { 4. Medical Homes/ } \\
\text { Medical Professionals }\end{array}$ & $\begin{array}{l}\text { This EHDI area includes the medical home and all } \\
\text { medical professionals responsible for the continuity } \\
\text { of care for children who have or are at-risk for } \\
\text { hearing loss. }\end{array}$ \\
\hline $\begin{array}{l}\text { 5. Loss to } \\
\text { Follow-Up }\end{array}$ & $\begin{array}{l}\text { This EHDI area includes key issues concerning loss to } \\
\text { follow-up during the EHDI process (screening to } \\
\text { identification to intervention). }\end{array}$ \\
\hline 6. Family Support & $\begin{array}{l}\text { This EHDI area includes the vital aspects of family } \\
\text { support services as well as both educational } \\
\text { materials and services that are culturally and } \\
\text { linguistically sensitive and readily available. }\end{array}$ \\
\hline $\begin{array}{l}\text { 7. Periodic Early } \\
\text { Childhood } \\
\text { Hearing Screening }\end{array}$ & $\begin{array}{l}\text { This EHDI area includes aspects of screening for } \\
\text { hearing loss in children who passed their initial } \\
\text { hearing screening but have a risk factor for hearing } \\
\text { loss, have an identified diagnosis that is associated } \\
\text { with hearing loss, or are being monitored for } \\
\text { late-onset hearing loss. }\end{array}$ \\
\hline $\begin{array}{l}\text { 8. Professional } \\
\text { Development }\end{array}$ & $\begin{array}{l}\text { This EHDI area represents education and professional } \\
\text { development for individuals who provide services } \\
\text { related to universal newborn hearing screening, } \\
\text { identification and intervention as well as } \\
\text { administrators who oversee various aspects of the } \\
\text { EHDI program. }\end{array}$ \\
\hline 9. Infrastructure & $\begin{array}{l}\text { This EHDI area encompasses all aspects of implement- } \\
\text { ing a UNHS program in a state. This includes, but is } \\
\text { not limited to, staffing at the state level, funding, } \\
\text { legislature/regulations, advisory committee, public } \\
\text { awareness, etc. }\end{array}$ \\
\hline $\begin{array}{l}\text { 10. Data System/ } \\
\text { Management }\end{array}$ & $\begin{array}{l}\text { This EHDI area deals with all aspects of collecting, } \\
\text { compiling, and analyzing data needed for follow-up, } \\
\text { reporting, program improvement, etc. }\end{array}$ \\
\hline
\end{tabular}


Table I. Cont.

\begin{tabular}{cc}
\hline EHDI Area & \multicolumn{1}{c}{ Description } \\
\hline 11. Quality Enhancement & $\begin{array}{c}\text { This EHDI area deals with how states use data, staff, } \\
\text { and other resources for quality improvement, } \\
\text { program evaluations, and projects. } \\
\text { This EHDI area includes not only the formal collaboration } \\
\text { that occurs, but also cooperation and systems } \\
\text { integration within the state systems and the private/ } \\
\text { community sectors. These collaborative efforts are } \\
\text { necessary for infants and young children with hearing } \\
\text { loss to receive appropriate audiological, medical, } \\
\text { educational, family support, and other needed services. }\end{array}$ \\
\end{tabular}

at a given point in time, not over time. This may lead to a snapshot look rather than a comprehensive analysis of the current EHDI system. Finally, SWOT analysis, as applied here, resulted in overlapping of themes within a category. For example, funding could be identified as a strength as well as a threat in many of the areas surveyed (see White \& Blaiser, 2011, for further comment regarding interpretation and application of findings).

\section{Strengths - Internal Factors}

Four frequently cited themes from the 12 areas emerged as strengths: communication, data management, advisory committee, and grant and funding opportunities (Table 2). Communication was cited as a strength in 10 out of the 12 areas (83\%). Specifically, EHDI coordinators reported that maintaining relationships with stakeholders, distributing newsletters and brochures with their state results, publishing provider directories, and establishing contracts (or memoranda of understanding) were essential to their success. An effective and efficient data management system was reported as essential to the state EHDI program in 9 out the 12 areas (75\%). Respondents indicated that these systems should be web-based and capable of generating useful reports. Highlights also included having epidemiologists on staff, sufficient information technologies (IT)/vendor support 24 hours/7 days a week, and the ability to receive electronic referrals. Having an EHDI advisory committee was reported as a strength in 8 out of the 12 areas (67\%). The advisory committee was viewed as an effective mechanism to ensure that good communication occurs among key stakeholders. Other strengths included having the EHDI coordinator serve on their state's Interagency Coordinating Council (ICC), various active committees working on state issues, and a mandate by the legislation to require an advisory committee. The fourth strength, grant support and funding opportunities, accounted for 8 out of the 12 areas 
Table 2. SWOT summary of the 12 EHDI areas

\begin{tabular}{|c|c|c|}
\hline & Positive/Helpful & Negative/Harmful \\
\hline 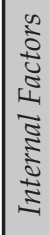 & $\begin{array}{l}\quad \text { Strengths } \\
\text { 1. Good Communication } \\
\text { 2. Effective Data Management } \\
\text { 3. Active Advisory Committees } \\
\text { 4. Grant and Funding } \\
\text { Opportunities }\end{array}$ & $\begin{array}{l}\text { Weaknesses } \\
\text { 1. Deficiencies in Data Management } \\
\text { 2. Staff Shortages/Lack of Qualified } \\
\text { Providers } \\
\text { 3. Barriers to Program Development }\end{array}$ \\
\hline 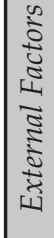 & $\begin{array}{l}\text { Opportunities } \\
\text { 1. Improved Communication and } \\
\text { Inter-Agency Relationships } \\
\text { 2. Improved Data Management } \\
\text { 3. Expanded Training } \\
\text { Opportunities }\end{array}$ & $\begin{array}{l}\qquad \text { Threats } \\
\text { 1. Limited Funding } \\
\text { 2. Insufficient Staff } \\
\text { 3. Insufficient Educational } \\
\text { Preparation } \\
\text { 4. Insufficient Legislation }\end{array}$ \\
\hline
\end{tabular}

(67\%). EHDI coordinators acknowledged the importance of federal funding sources and appreciated opportunities to apply for grants and other support. Some also reported reimbursement for hearing aids and other related services as a significant strength.

\section{Weaknesses-Internal Factors}

Three frequently cited themes from the 12 areas emerged as weaknesses: data management, staff shortages/lack of qualified providers, and program barriers (see Table 2). Problems with the existing data management system were reported in 10 out of the 12 areas (83\%). Some of the responses included not having a structured data management system, the system not being webbased or electronic, lack of access to the system by providers in the state, inconsistent data entry, and no back-up or emergency system. Lack of staff and qualified providers was another consistent weakness reported in 10 out of the 12 areas (83\%). Reported issues included staff turnover, not enough staff to effectively run the EHDI program, vacant positions that cannot be filled due to a lack of qualified providers, and lack of shared positions. Another weakness highlighted in 8 of the 12 areas $(67 \%)$ included program barriers, such as concerns about program sustainability, lack of quality assurance projects, need for legislative mandates, geographic barriers (i.e., too many rural or remote areas to service), and lack of protocols and procedures.

\section{Opportunities-External Factors}

When EHDI coordinators were asked to identify opportunities, three frequently cited themes emerged: communication and relationships, data 
management systems, and professional training and education (see Table 2). Opportunities for better communication and relationships were reported in 10 out of the 12 areas (83\%). Specifically, respondents reported that working groups focused on the various components of EHDI were helpful as was collaboration with university programs for training, research, and grant writing. Respondents stated that in 9 of the 12 areas (75\%) there were opportunities to improve data management systems. Those respondents reported that they were in the process of upgrading their systems in ways that would reduce or eliminate double data entry, link their data system to other state programs, or allow them to work with the National Initiative for Children's Healthcare Quality (NICHQ) Learning Collaborative to improve their data system. Finally, professional training and education emerged as an opportunity in 9 out of the 12 areas (75\%). With video conferencing, webcasts, personalized education tools, and resources provided through the NICHQ Learning Collaborative, EHDI coordinators felt there were many opportunities that could be pursued to enhance professional training and education.

\section{Threats-External Factors}

Of the 12 areas of EHDI examined, potential loss of funding was the only theme that EHDI coordinators unanimously reported as a significant threat to their programs (see Table 2). They cited budget cuts, fiscal crises, and concerns about re-authorization of federal legislation. Other frequently cited themes that emerged were limited staff, limited training and education, and lack of legislation. Respondents in 11 of the 12 areas (92\%) cited limited staff as a major threat to the program. State EHDI coordinators also expressed concerns about a lack of qualified providers, lack of provider motivation and interest in their jobs, and lack of EHDI staff. Respondents in 5 out of the 12 areas (42\%) identified limited training and education as threats. Respondents specifically noted that it is difficult to reach all of them due to travel restrictions, high cost of trainings and conferences, and a variety of professionals to train within EHDI (e.g., audiologists, primary care providers, otolaryngologists, early interventionists, speech-language pathologists, teachers). Finally, respondents in 5 out of the 12 areas (42\%) reported problems with existing mandates or not having EHDI-related legislation in place.

\section{TOWS Analysis Matrix and Recommendations}

By applying the TOWS matrix to the themes identified in the SWOT analysis, specific recommendations, "or strategic options," were derived from the internal and external environmental factors identified by the EHDI coordinators. By systematically pairing the four categories of the SWOT analysis, recommendations were derived to maximize resources and effect positive 
change. Based on an analysis of all 12 areas, the following emerged as recommendations to strengthen EHDI programs:

- Examine effective and efficient web-based data management systems; provide and/or share information with EHDI programs, vendors, and federal and state agencies; and take advantage of funding opportunities to develop these systems in each state.

- Build collaborative relationships through a variety of communication outlets, educational venues, advisory groups, and committees.

- Develop protocols and procedural guidelines to enhance data collection, communication, and personnel preparation.

- Utilize communication, relationships, and professional networks to work with state and federal lawmakers to secure mandates that support needed outcomes.

- Draw on the EHDI advisory committee and outcome data to prioritize objectives in ways that maximize state resources and allocation of personnel.

- Strengthen data reporting systems to impact legislation and funding decisions at both the state and federal levels.

- Engage university training programs in ways that create preservice and inservice educational opportunities for physicians, nurses, audiologists, speech-language pathologists, early interventionists, and teachers.

- Require state agencies, service providers, and related organizations to be accountable to relevant standards and protocols.

Data from this study indicate that many states have found solutions to barriers and have turned program weaknesses into strengths. State EHDI programs are increasingly successful in their efforts to ensure that infants are screened by 1 month of age, diagnosed no later than by 3 months of age, and have access to appropriate hearing technology and intervention services no later than 6 months of age, as recommended by the Joint Committee on Infant Hearing $(\mathrm{JCIH}, 2007)$. Furthermore, a growing number of systems are in place to identify and provide intervention for children who have later onset hearing loss. Through collaboration, improved infrastructure, and successful personnel preparation, many states have the elements that underpin a successful EHDI program. It is hoped that through sharing of resources, networking, and cooperative relationships the nation will continue to build EHDI systems that provide the information, resources, and services needed to optimize goals and outcomes for each child with hearing loss.

\section{References}

Centers for Disease Control and Prevention (CDC). (2010). Annual data: Early hearing detection and intervention (EHDI) program. Retrieved May 24, 2011, from http://www.cdc.gov/ncbddd/hearingloss/ehdi-data.html. 
Ghazinoory, S., Abdi, M., \& Azadegan-Mehr, M. (2011). SWOT methodology: A state-of-the-art review for the past, a framework for the future. Journal of Business Economics and Management, 12(1), 24-48.

Joint Committee on Infant Hearing (JCIH). (2007). Year 2007 position statement: Principles and guidelines for early hearing detection and intervention programs. Pediatrics, 120(4), 898-921.

Russ, S. A., White, K., Dougherty, D., \& Forsman, I. (2010). Preface: Newborn hearing screening in the United States: Historical perspective and future directions. Pediatrics. 126(1), S3-S6.

Weihrich, H. (1982). The TOWS matrix-a tool for situational analysis. Long Range Plan, 15, 54-66.

Weihrich, H. (1998). Daimler-Benz's move towards the next century with the TOWS matrix. In: Dyson, R., \& O'Brien, F. (Eds.), Strategic development: methods and models (pp. 69-79). Indianapolis, IN: Wiley.

White, K., \& Blaiser, K. (2011). Strategic planning to improve early hearing detection and intervention programs. The Volta Review, 111(2), 83-108.

White, K. R., Forsman, I., Eichwald, J., \& Muñoz, K. (2010). The evolution of Early Hearing Detection and Intervention Programs in the United States. Seminars in Perinatology, 34(2), 170-179. 
